STATE OF ILLINOIS

DEPARTMENT OF REGISTRATION AND EDUCATION

DIVISION OF THE

NATURAL HISTORY SURVEY

STEPHEN A. FORBES, Chief

\begin{tabular}{lll} 
Vol. XIV. BULLETIN Article VII. \\
\hline
\end{tabular}

\title{
CODLING-MOTH INVESTIGATIONS OF THE STATE ENTOMOLOGIST'S OFFICE
}

$1915,1916,1917$

BY

PRESSLEY A. GLENN

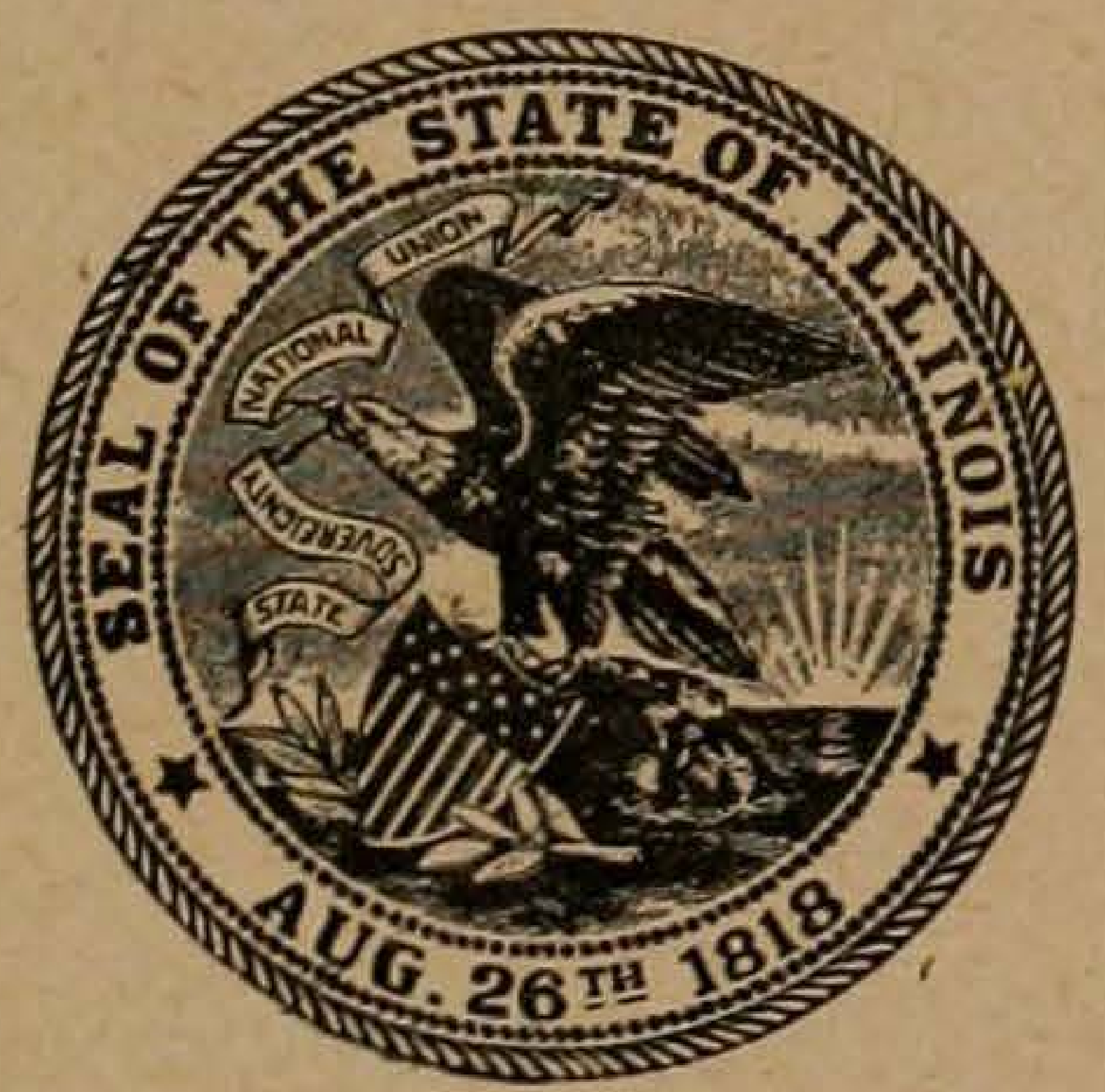

PRINTED BY AUTHORITY OF THE STATE OF ILLINOIS

URBANA, ILLINOIS

August, 1922 
STATE OF ILLINOIS

DEPARTMENT OF REGISTRATION AND EDUCATION

W. H. H. Miller, Director

\section{BOARD OF}

\section{NATURAL RESOURCES AND CONSERVATION}

W. H. H. Miller, Chairman

William Trelease, Biology

John M. Coulter, Forestry Rollin D. Salisbury, Geology William A. Noyes, Chemistry
JoHN W. ALvord, Engineering

KendRIC C. BABCOCK, Representing the President of the University of Illinois

THE NATURAL HISTORY SURVEY DIVISION

Stephen A. Forbes, Chief

$(64922-1200-6-22)$

PHILLIPS BROS. PRINT. 


\section{CONTENTS}

Introductory statement $\ldots \ldots \ldots$

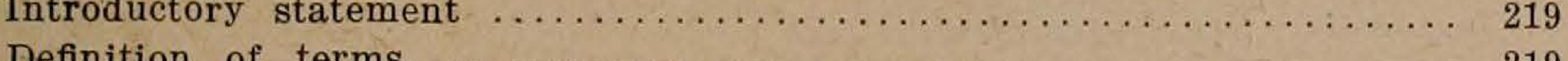

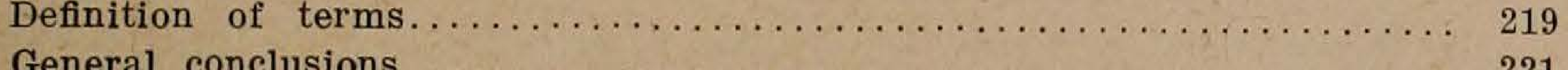

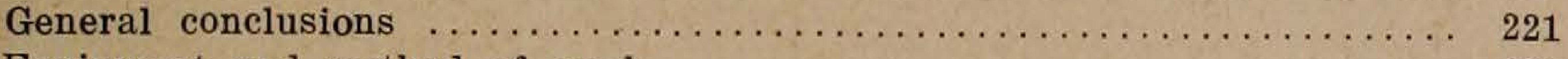

Equipment and method of work ........................ 223

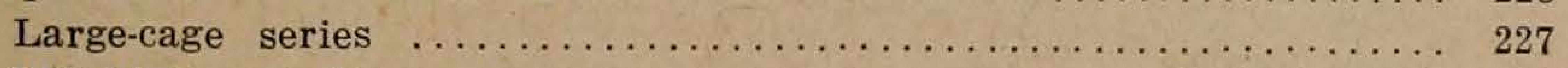

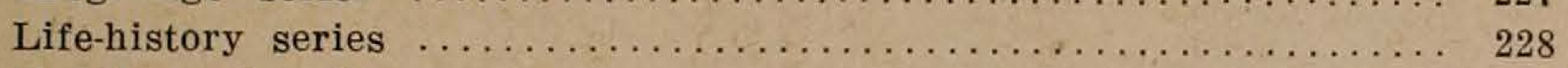

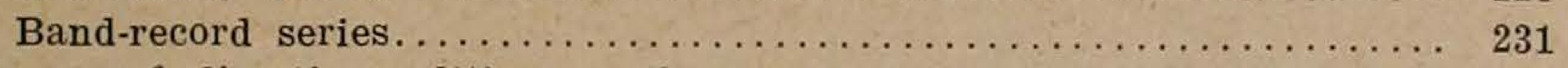

Influence of climatic conditions on the egg, larva, and pupa of the codling-

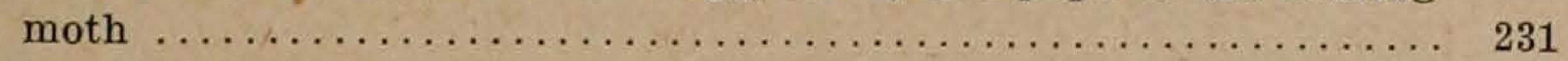

Zones of temperature............................... 232

General procedure in the study of temperature relations......... 233

Relation of temperature to development of the egg............. 234

Relation of temperature to development of the larva............. 239

Relation of temperature to development of the pupa............ 246

Conclusions from the foregoing discussion .................. 252

Observations on the life history of the codling-moth in 1915, 1916, and 1917. 253

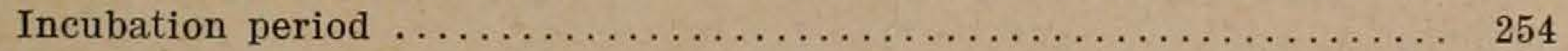

Larval period:

Transforming lavæ............................ 254

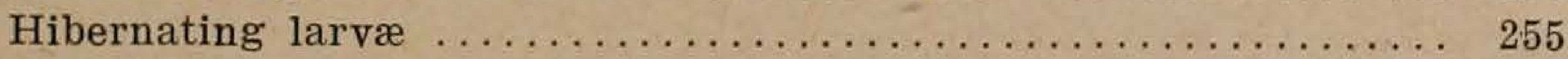

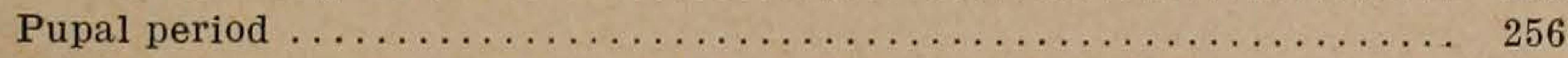

Combined egg, larval, and pupal periods.................. 256

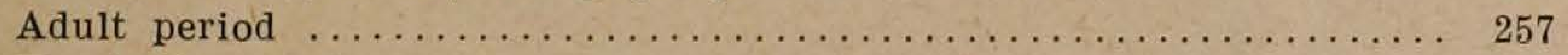

Total life-period $\ldots \ldots \ldots \ldots \ldots \ldots \ldots \ldots \ldots \ldots \ldots \ldots \ldots \ldots \ldots \ldots \ldots \ldots \ldots \ldots \ldots \ldots \ldots \ldots \ldots \ldots, 257$

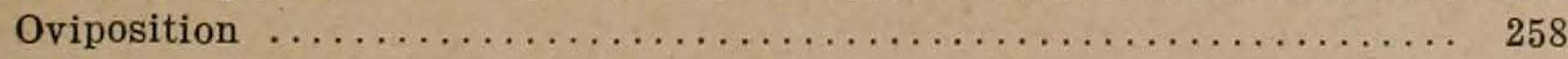

Relation of temperature to length of development period.......... 258

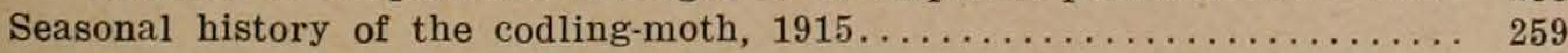

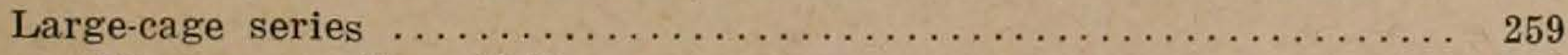

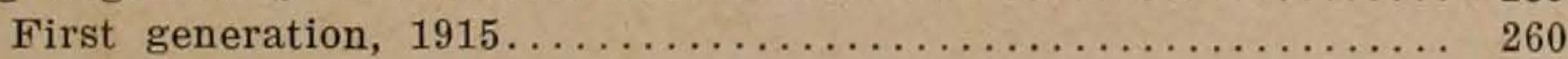

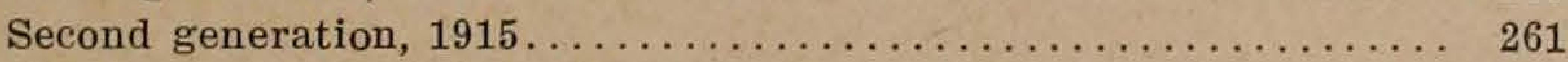

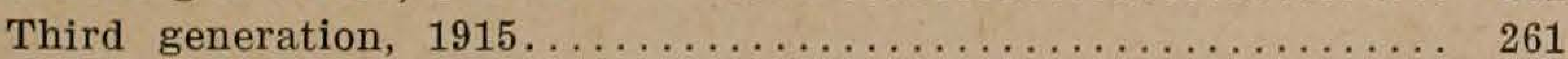

Band collections:

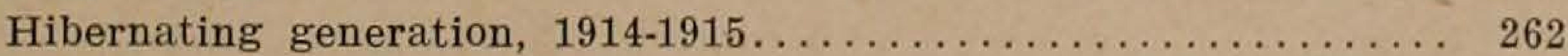

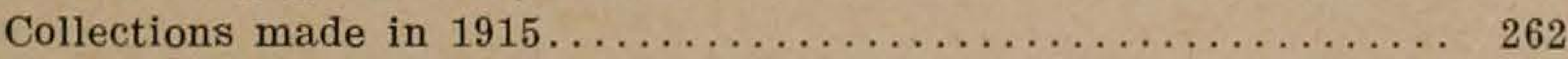

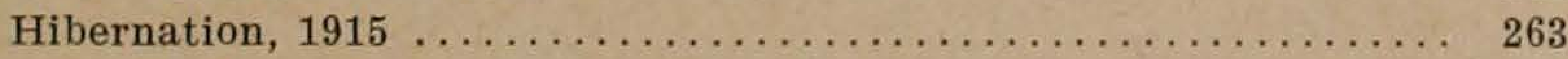

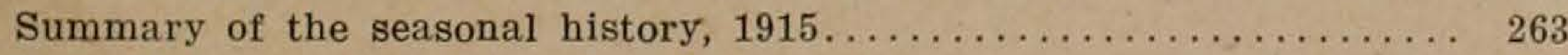

Records for other points than Olney ..................... 264

General remarks on the season......................... 264 
PAGE

Seasonal history of the codling-moth, $1916 \ldots \ldots \ldots \ldots \ldots \ldots \ldots \ldots \ldots \ldots \ldots$

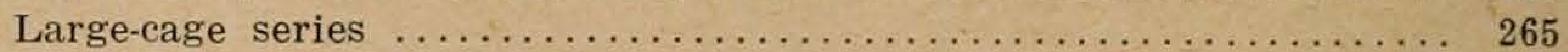

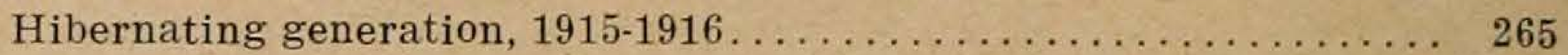

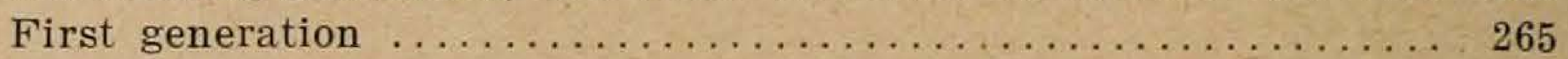

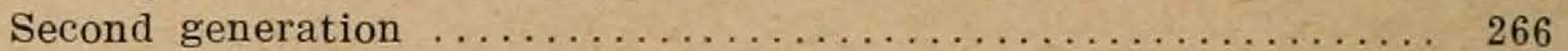

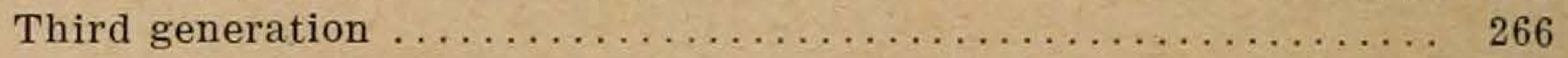

Band-collections:

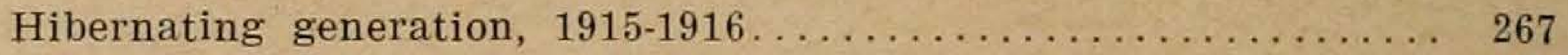

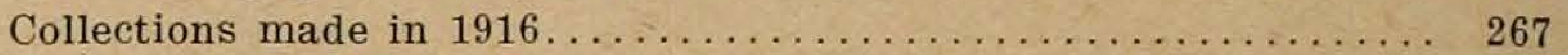

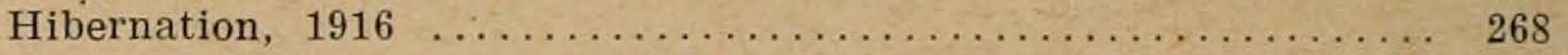

Summary of the seasonal history, $1916 \ldots \ldots \ldots \ldots \ldots \ldots \ldots \ldots \ldots \ldots$

Records for several localities........................ 271

Seasonal history of the codling-moth, $1917 \ldots \ldots \ldots \ldots \ldots \ldots \ldots \ldots \ldots \ldots \ldots 272$

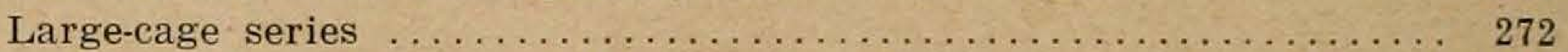

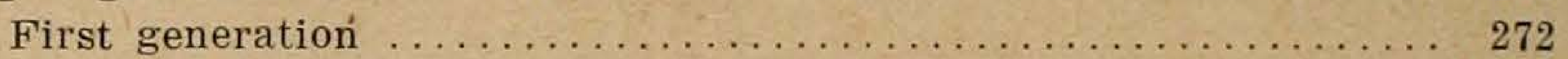

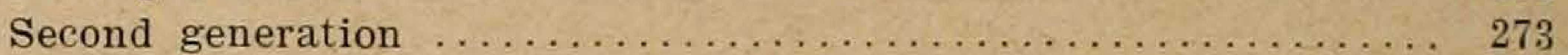

Third generation $\ldots \ldots \ldots \ldots \ldots \ldots \ldots \ldots \ldots \ldots \ldots \ldots \ldots \ldots \ldots \ldots \ldots$

Band collections:

Hibernating generation, $1916-1917 \ldots \ldots \ldots \ldots \ldots \ldots \ldots \ldots \ldots \ldots \ldots \ldots \ldots$

Collections made in 1917 (see Table 37 ) . . . . . . . . . . . . . 273

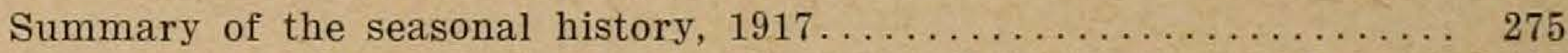

Relation of temperature to the seasonal history of the codling-moth..... 275

Method of forecasting dates when the different stages of the several gener-

ations may be expected to appear at any given place in a normal season. 281

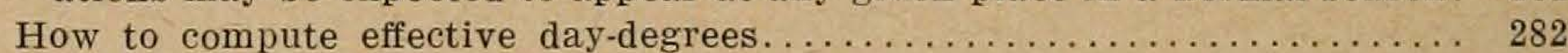

Relation of temperature to time when larvæ hibernate . . . . . . . . . 283

Relation of humidity and evaporation to the development of the codling-

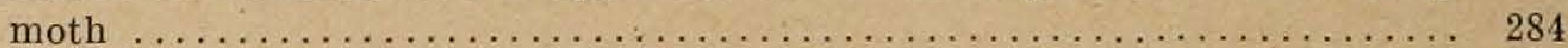

Spray program for the codling-moth..................... 288

Figures (8), Graphs (7), Tables (51), Charts (3). 
Akticle VII.-Codling-moth Investigations of the State Entomologist's Office, 1915, 1916, and 1917. By P. A. GLenN, Chief Inspector of the Division of Plant Industry, State Department of Agriculture.

\section{INTRODUCTORY STATEMENT}

To secure accurate data in regard to the life history and the seasonal history of the codling-moth in Illinois, and the relation of climatic conditions to the rate of development and the time of appearance of the various stages of the insect, an investigation was begun in the spring of 1915 under the direction of Doctor S. A. Forbes, then State Entomologist. The principal station was established at Olney in charge of the writer, at that time and during the whole period of this investigation on the staff of the Entomologist.* Other stations were at Ozark, about 80 miles south and 45 miles east of Olney, in charge of Mr. L. M. Smith; and at Plainview, 40 miles north and 100 miles west of Olney, and at Springfield, 70 miles north and 90 miles west of Olney, in charge of Mr. W. P. Flint.

The station at Olney was equipped with large, screen-wire cages covering entire trees, a field laboratory and all necessary small cages for the observation of individual insects in all their stages, a self-recording thermograph, a self-recording hygrograph, a Livingston porous cup atmometer for measuring evaporation and a rain gage (Fig. 8, p. 230), standard maximum and minimum thermometers, and a sling psychrometer for checking daily the records of the self-recording instruments. The other stations were provided with instruments for keeping a record of climatic conditions.

At Olney a continuous daily record was kept of the history of all the stages of the insect. At the other stations band records and other data were recorded such as would enable us to follow the main features of the seasonal history of the insect.

\section{Definition of TERMS}

To avoid confusion resulting from a misunderstanding of terms, the following definitions are given:

The term "stages of the codling-moth" refers to the four distinct periods in the life of the insect: the egg, the larva, the pupa, and the adult.

- As a consequence of the passage of the Civil Administrative Code in 1917, the writer was transferred to the State Department of Agriculture, and the major part of this report has been written since he became a member of this department, the State Entomologist's offlce having been by the same law merged in the Natural History Survey of the State, with Dr. Forbes as its chief. This double relation has not, however, affected the character of this report, which has been prepared under the supervision of Dr. Forbes, and with a view to its publication as a survey bulletin. 
The term "generation" is used to designate a group of insects in all the four stages from egg to adult, all equally removed by descent from a given insect or group of insects, except that the term "hibernating generation" is used for all the insects which hibernate, regardless of the generations to which they belonged the preceding year.

The term "brood" is used to designate all the individuals of one of the stages of a generation, as, brood of eggs, brood of larvæ, etc. The terms "first brood", "second brood", and "third brood" are used to designate the eggs, larvæ, pupæ, or adults of successive generations.

The "theoretical zero of development" is the temperature at which development begins when the temperature is rising and at which it ceases when the temperature is falling.

The "degree of maximum rate of development" is the temperature at which development proceeds most rapidly.

The "day-degree" is the unit used for measuring accumulations of temperature, and is equivalent to a temperature of one degree maintained for 24 hours.

"Effective day-degrees" are day-degrees above the zero of development, after necessary corrections have been made for retardation due to temperatures above the degree of maximum rate of development. This correction is made by subtracting twice the day-degrees above the degree of maximum rate from the total day-degrees above the zero of development.

The codling-moth passes the winter in the larval stage. The overwintering larvæ belong principally to the second and third generations of the preceding year, but for convenience in discussing the seasonal history we designate them as the hibernating generation. The generations which descend from the hibernating generation are designated as the "first generation", the "second generation", etc. In accordance with the foregoing definitions we have the following relations of generations and broods :

The hibernating generation, consisting of

the hibernating larvæ, the first brood of pupæ, and the first brood of adults.

The first generation, consisting of the first brood of eggs, the first brood of larvæ, the second brood of pupæ, and the second brood of adults.
The second generation, consisting of

the second brood of eggs, the second brood of larvæ, the third brood of pupæ, and the third brood of adults.

The third generation, consisting of the third brood of eggs, the third brood of larvæ, etc.

In the case of insects which pass the winter as adults, the number of the broods will agree with the number of the generations to which they belong, but in the case of those that pass the winter in the egg, larval, or pupal stage, these numbers will not agree in all the broods. 


\section{General Conclusions}

The following conclusions have been reached as the results of this investigation:

There are normally in the southern half of the state one complete generation, a nearly complete second generation, and a partial third generation of the codling-moth each year. In the northern part of the state there are but two generations.

In the latitude of Olney, larvæ of the first generation normally begin to enter the fruit about May 19; larvæ of the second generation, July 4; and larvæ of the third generation, August 12.

The data at hand are not sufficient to enable us to say definitely just how much allowance must be made in the time of appearance of the broods for differences of latitude, but in general, the first pupæ, moths, eggs, and young larvæ appear two or three days earlier at Ozark and two or three days later at Springfield than at Olney; and second and third broods of larvæ appear about a week earlier at Ozark and a week later at Springfield than at Olney.

The pupal period varies from 8 to 46 days, depending mainly upon the temperature. The period of the first brood is much longer than that of the second and third broods. The first brood period averages 30 days, the second 10.2 days, and the third 11.4 days.

The life of the male adult in confinement varies from 3 days to 24 days, the average being 10.5 days; the life of the female adult in confinement varies from 2 days to 27 days, the average being 13.4 days.

The female moth begins to lay eggs in from one to five days after emergence, the majority of the eggs being laid between the second and the fifth days, and oviposition is usually completed within ten days.

The egg period varies from 4 to 15 days, depending mainly upon the temperature. The average was 8.1 days for the 4956 eggs under observation.

The larval period varies from 18 to 47 days for transforming larvæ and from 244 to 302 for hibernating larvæ, the former averaging 26.4 days and the latter 264.8 days. The feeding period varies from 14 to 43 days for transforming larvæ and from 16 to 54 for hibernating larvæ, the average for the former being 24.7 and for the latter 29.3. The larval period in the cocoon varies for transforming larvæ from one to eleven days, and for hibernating larvæ from 206 to 275 days, the average for transforming larvæ being 4.2 and for hibernating larvæ 206.1 days.

The total life-period varies from 41 to 70 days for individuals whose larvæ transform, the average being 54.2, and from 287 to 332 days for individuals whose larvæ hibernate, the average being 313 days.

Larvæ begin to go into hibernation about August 1, and during the month the percentage of maturing larvæ which hibernate increases more or less rapidly, depending upon temperatures, until September 1, after which practically all larvæ hibernate. 
Temperature is the predominating factor, so far as weather conditions are concerned, in determining the rate of development and the time of the appearance of the various stages.

Development begins in the spring as soon as the temperature in the warmer parts of the day rises above 52 degrees $F$., and the rate of development increases as the temperature rises,* until the degree of the maximum rate of development is reached, which for the egg is about 88 , for the larva 85 , and for the pupa 87 degrees $F$. The pupa will not develop at temperatures below 52 degrees, but the egg and larva will develop at temperatures above 50 .

Temperatures above the degree of maximum rate of development retard development, and the retardation appears to be proportional to the number of degrees above the degree of the maximum rate, so that the rate of development at one, two, three, etc., degrees above the degree of the maximum rate is the same as one, two, three, etc., degrees below that degree.

There is a fairly constant relation between the accumulation of effective day-degrees and the time of appearance of the different broods. For practical purposes, 50 degrees F. may be used as the zero of development and 86 degrees as the degree of maximum rate of development, and the effective day-degrees for any day may be determined by finding the average temperature above 50 degrees and subtracting from it twice the average temperature above 86 degrees.

It requires an average of 163,673 , and 265 effective day-degrees to complete the development of the egg, larval, and pupal stages, respectively, and an average of 1101 for the development of an individual through the three stages when 50 is taken as the zero of development and 88, 85, and 87 as the degrees of maximum rate of development for the egg, larva, and pupa, respectively. The number of effective day-degrees which accumulate from a certain stage in the development of one generation to the same stage in the development of the succeeding generation varies in round numbers from about 1000 to 1600 , the average being about 1195 , when 50 is taken as the zero and 86 as the degree of maximum rate.

The first larvæ of the first, second, and third generations enter the fruit when the accumulations of effective day-degrees have reached about 550, 1550, and 2550 day-degrees, respectively. $\dagger$

The seasonal history of the codling-moth for a normal year may be determined approximately in any locality by ascertaining the normal total accumulation of effective day-degrees for each day in the season in that locality.

- This statement is not strictly true, for with each unit of change in temperature, the rate of development is affected more at temperatures near the zero of development, and less at temperatures near the degree of maximum rate of development than at intermediate temperatures, but these variations from the rule are so slight that they may be disregarded for the purposes of this paper.

$\leftarrow$ The statements with regard to temperature relations may possibly not apply to places differing widely in latitude or altitude from olney, Illinois. 
The actual accumulation for any date during different seasons may vary widely from the normal accumulation.

By comparing the actual accumulation at any date during a given season with the normal accumulation for that date, the approximate number of days which development has been retarded or hastened on that date may be determined; and, by keeping track of the number of effective day-degrees that accumulate as the season advances, one may foretell, approximately, several days in advance, the dates when the larvæ of the different generations will begin to enter the fruit.

\section{EQUipment AND Methods of Work}

Aside from the large cages and the weather instruments previously mentioned, cages were devised for observing pupation of larvæ, emergence of moths, oviposition, and length of life of adults.

For observing pupation, cages such as those illustrated in Figures 1 and 2, were found to be most satisfactory. They consisted of two blocks of wood and a strip of celluloid, each $43 / 4$ inches long and $2 \frac{1}{2}$ inches wide. On the face of one of the blocks, strips of wood or heavy paper about $1 / 8$ inch thick were tacked along the end and longitudinally through the middle of the block, to hold the blocks far enough apart to permit the larvæ to crawl in between them. A strip of corrugated strawboard split through the middle so as to leave half of the corrugations on each face was placed, with the split surface up, on the block on each side of the center strip so as to cover the remaining face of the block. The other block was placed on the top of this with a strip of celluloid between, and the blocks were held together by small hooks and eyes fastened to the edges of the block, or by rubber bands - the latter proving the more satisfactory. There was room in each cage for about 50 larvæ. Smaller cages for single larvæ or a small number of larvæ were made on the same principle without blocks of wood.

When it was desired to determine the dates of pupation of larvæ collected from bands, they were confined in a lantern globe with one or more of the larger pupa-cages. Thus enclosed, the larvæe crawled into the little cells and spun their cocoons, and were thus arranged in very convenient order for further observation. By the use of sandpaper, the celluloid was roughened longitudinally through the center of the block, and after the larvæ were settled the number of each was entered on the celluloid beside it. (Fig. 2.)

Usually, transforming larvæ spun so thin a web on the exposed side of the cocoon that pupation could be observed without disturbing them, but hibernating larvæ spun a thicker web, and to observe them it was necessary to tear away a part of the web next to the celluloid every time an observation was made. The most convenient instrument for doing this was a steel crochet-needle with the hook ground away so as to leave only a small part of the barb. The cages above described will be referred to as pupa cages. 


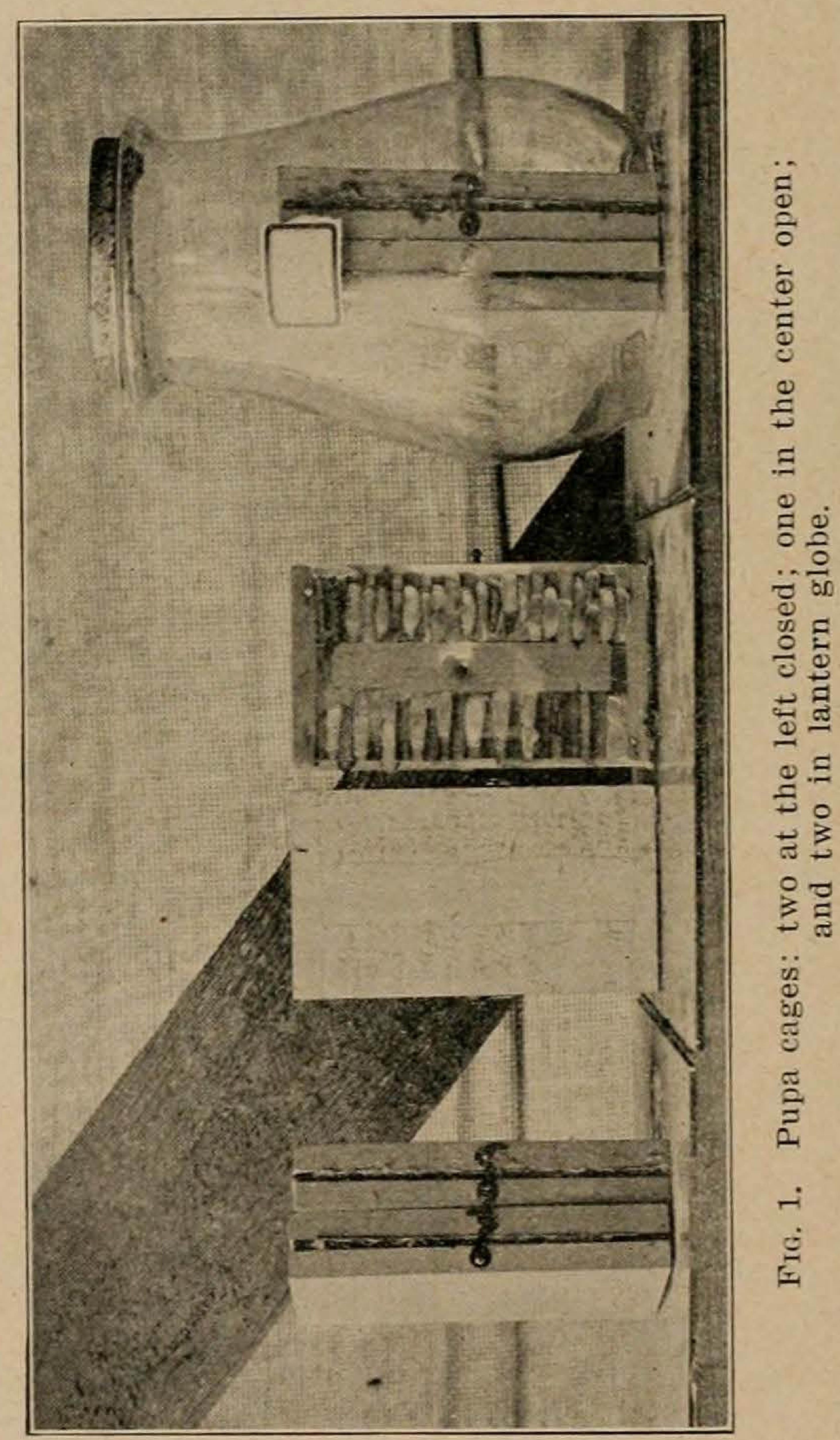




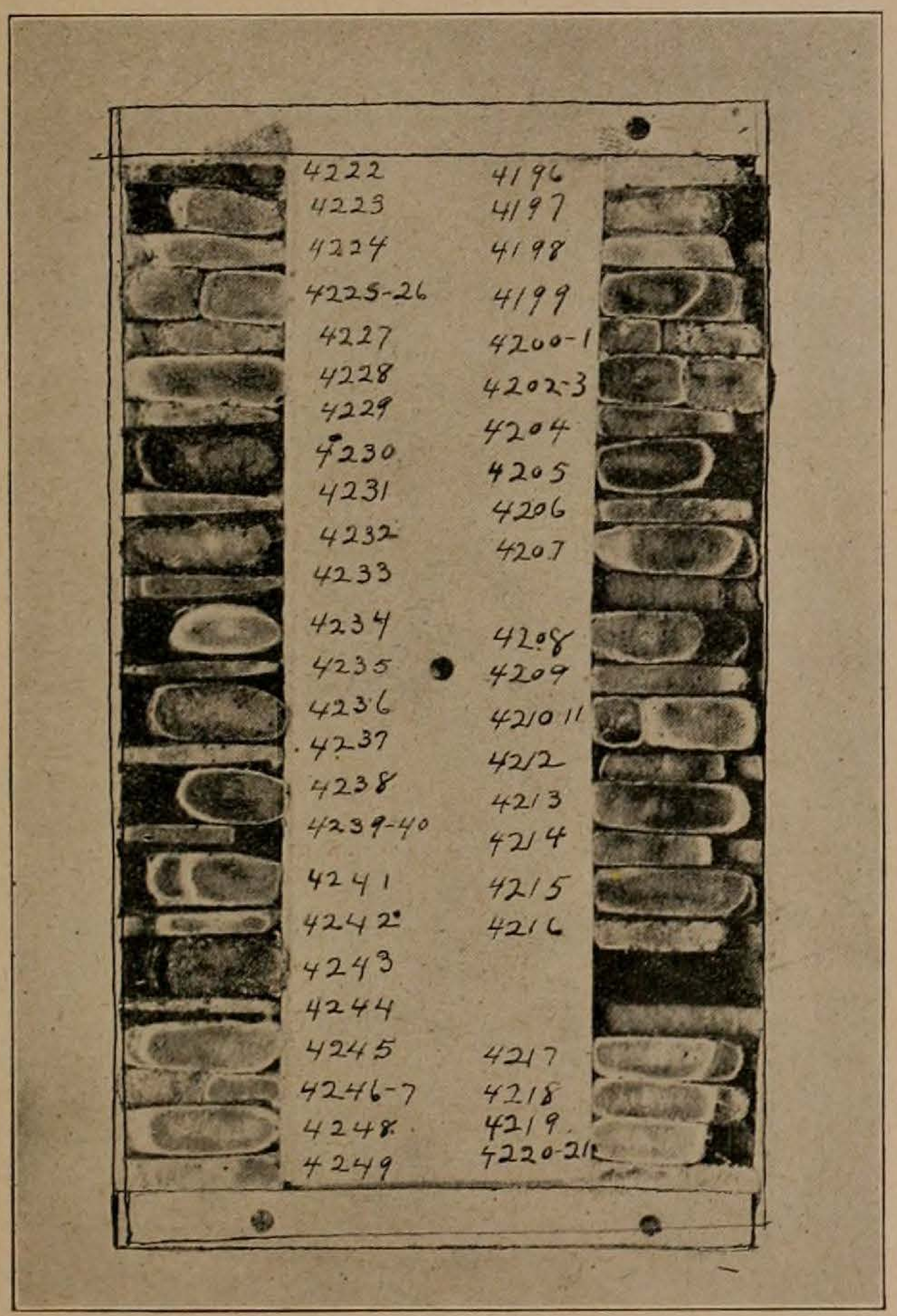

FIg. 2. Pupa cage open, showing arrangement of larvæ and method of numbering. 
When it was desired to observe only the emergence of moths, larvæ were placed in a $4-o z$. condiment jar containing a bundle of strips of strawboard. (Fig. 3.) These cages will be designated as emergence cages.

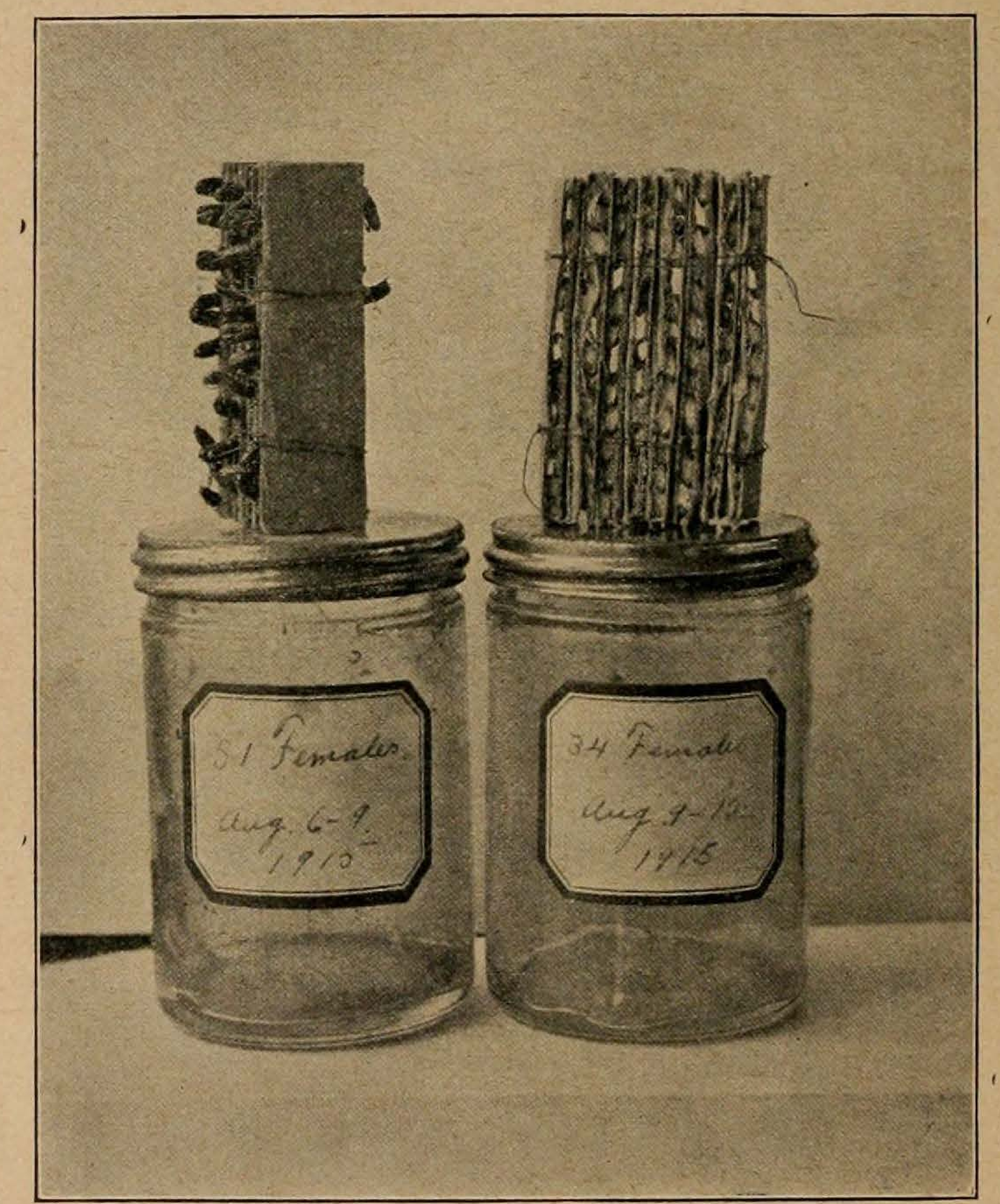

Fig. 3. Emergence cages. Strips of strawboard removed from jars to show arrangement of cocoons and pupa cases.

To secure eggs and note length of life of adults, pairs of moths were confined in cages consisting of a cylinder of screen-wire closed at one end with screen-wire and at the other end with an apron of cheese-cloth which permitted the cage to be fastened over the top of a branch of a tree. (Fig. 4.) These will be called oviposition cages. 
The experiments were carried on in three series, kept as nearly independent as possible so that each would serve as a check on the others. I shall designate them as the large-cage series, the life-history series, and the band-record series.

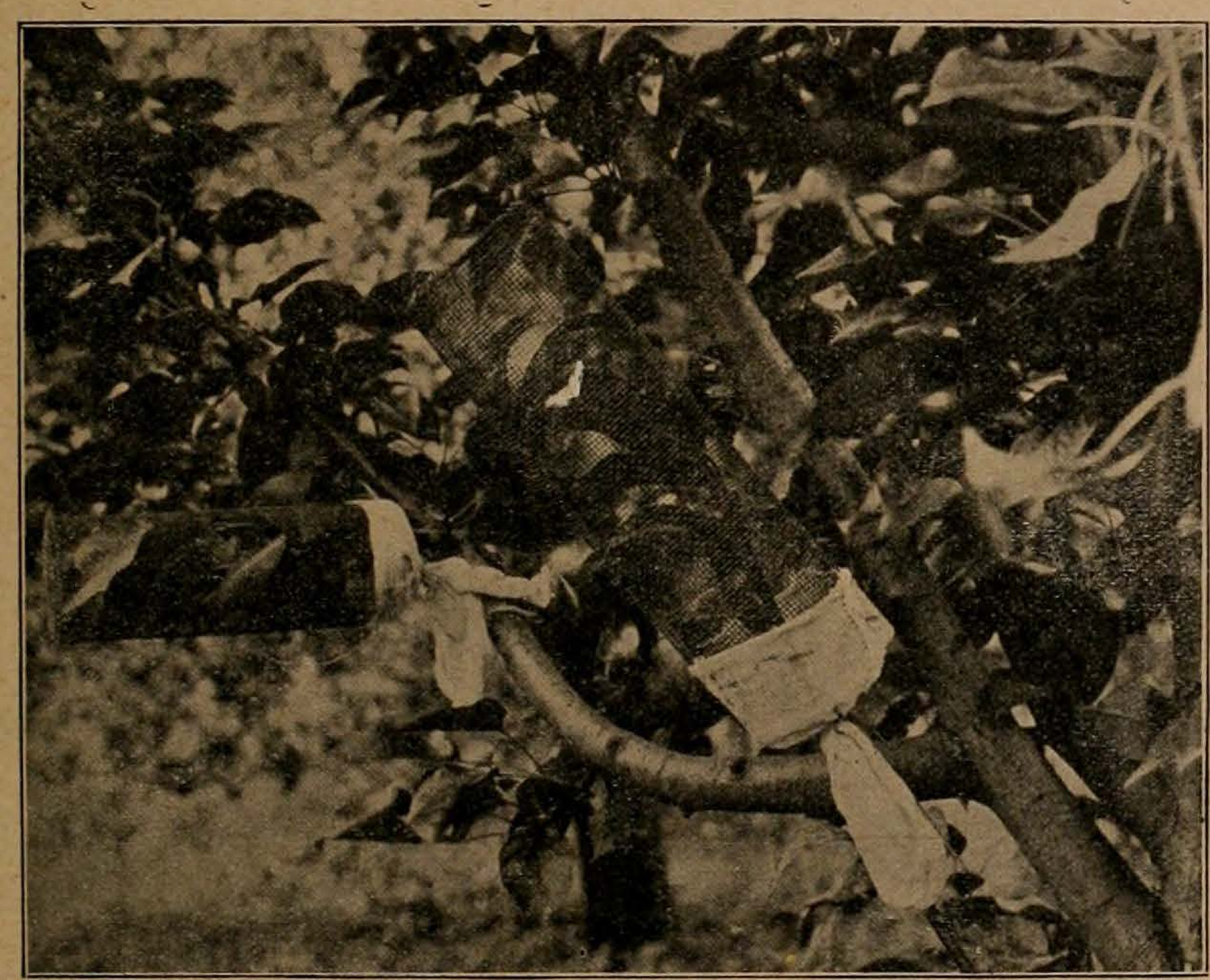

Fig. 4. Two oviposition cages in place on tree.

\section{LARGE-CAGE SERIES}

Cages used in this series were large enough to cover entire trees, the largest cages being 18 feet square and 18 feet high. (Fig. 5, 6, and 7.) The plan was to use two cages each for the first and the second generations and one cage for the third generation-if a third generation appeared.

The framework of the cages, consisting of $2 \times 4$ scantling, was erected over the trees before they were in bloom, and wire cloth, 12 wires to the inch, was tacked over the frame as soon as the time of full bloom was past. Each of the cages had a tight-fitting screen-door provided with hasp and padlock.

The trees thus screened in were examined very carefully for larvæ and two inches of the soil in each cage was removed.

The first moths to emerge from the hibernating larvæ were placed in cage No. 1, and the last moths to emerge in cage No. 2, to rear the first and the last individuals of the first generation; the first moths reared 
in cage No. 1 were liberated in cage No. 3 and the last moths reared in cage No. 2 were liberated in cage No. 4, to rear the first and the last individuals of the second generation; and the moths reared in cage No. 3 were liberated in cage No. 5, to rear the third generation. In this way it was possible to ascertain approximately the dates when each generation began and ended.

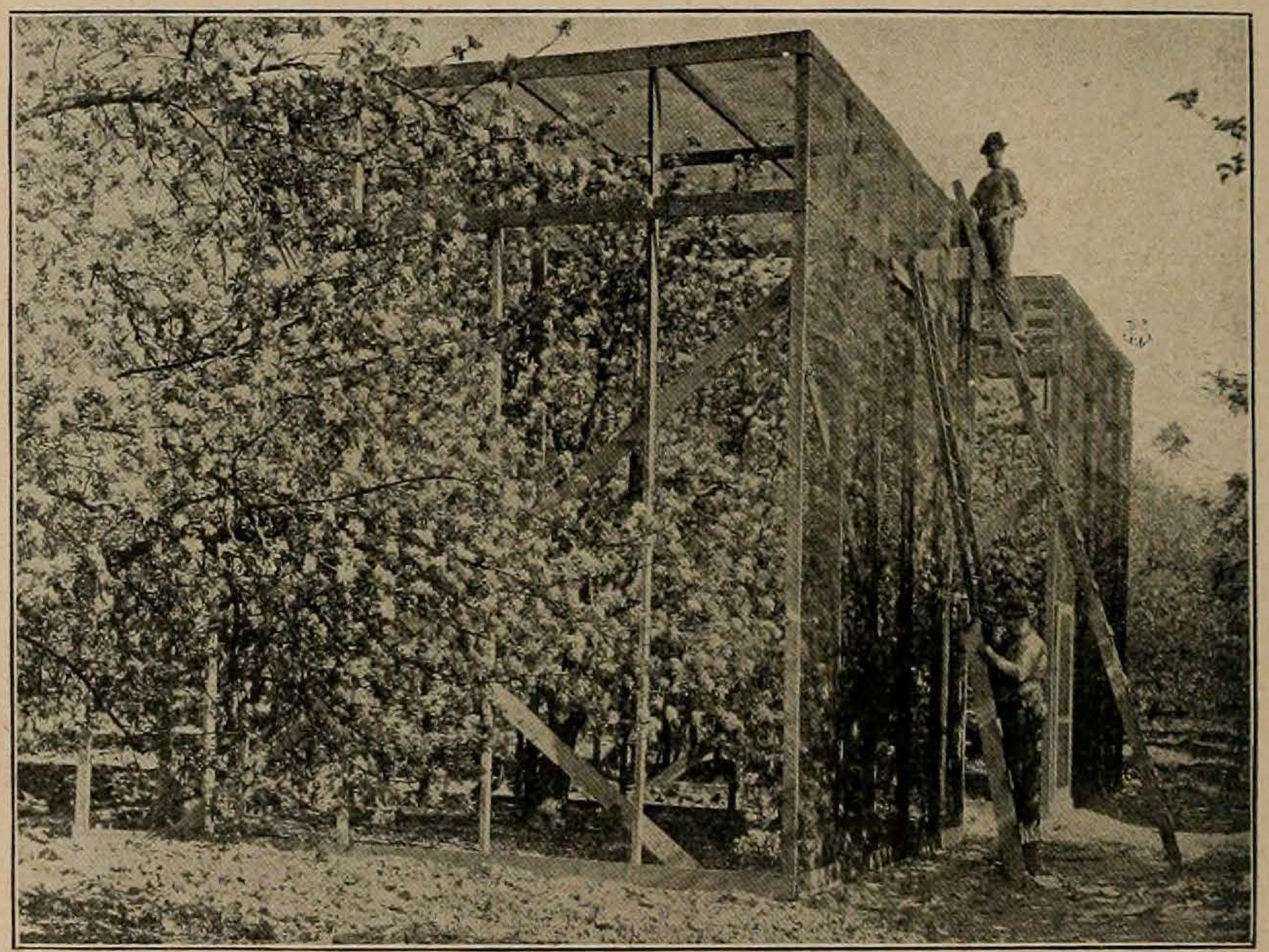

Fig. 5. Large screen-cage in course of construction.

\section{LIFE-HISTORY SERIES}

In the life-history series, pairs of adults of each of the broods were enclosed in oviposition cages (Fig. 4), and the number of eggs laid daily by each female and the dates of the death of the adults were noted.

The eggs obtained were numbered and allowed to remain on the trees until the black spot appeared. They were then removed to small vials on the bits of leaf or bark on which they had been laid, and, as they hatched, the larvæ were transferred to apples with a camel-hair brush, some to apples on the trees and others to picked fruit. The apples were tagged and each was given a number corresponding to that of the egg, and when they showed conspicuous signs of worminess they were transferred to jars, in which were placed strips of corrugated strawboard or small pupal cages in which the larvæ might spin up. The dates were noted when the larvæ left the apple, when they pupated, and when the adults emerged. 


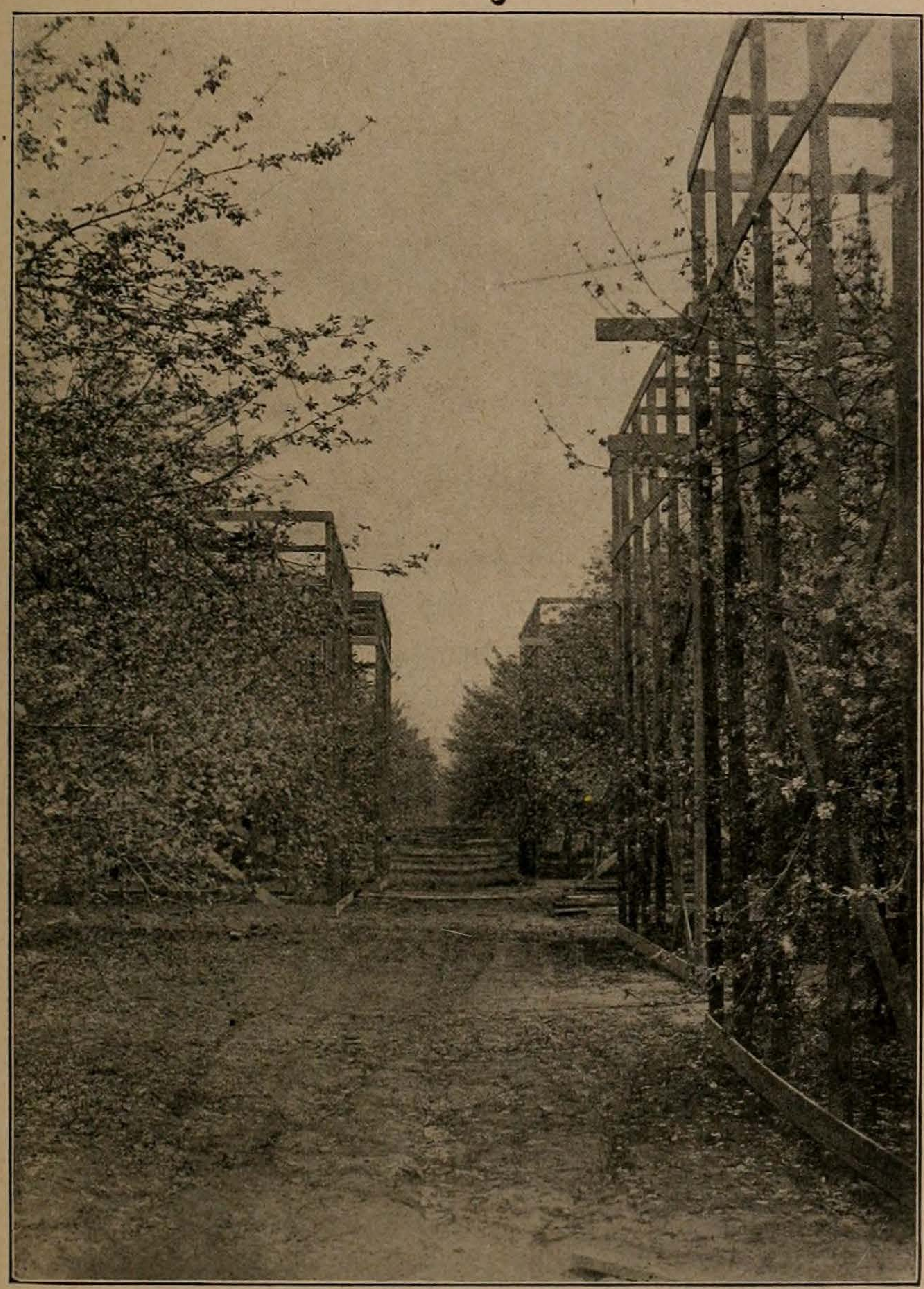

Fig. 6. View of orchard showing screened trees. 


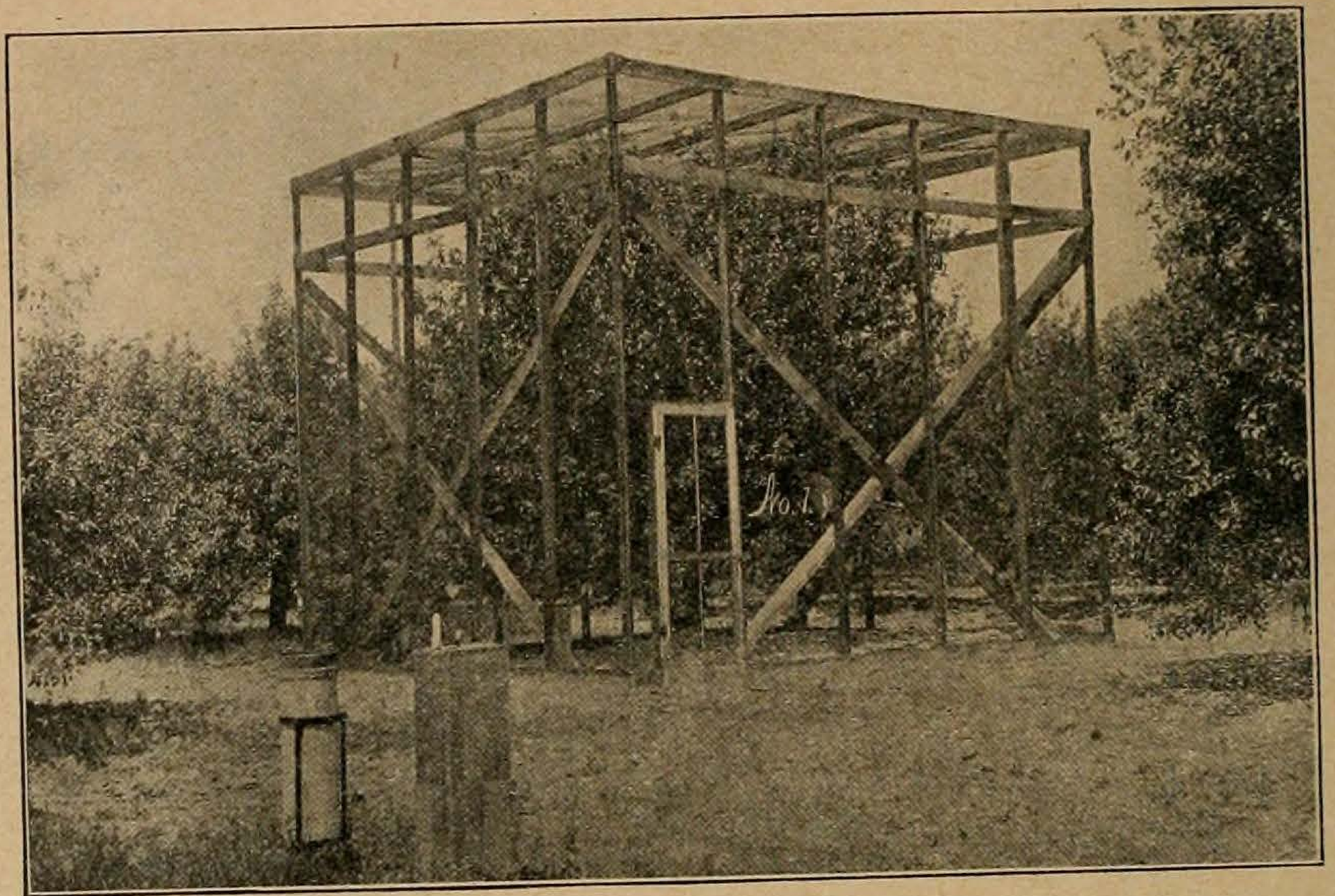

FIG. 7. Large cage completed. Rain-gage and atmometer in foreground.

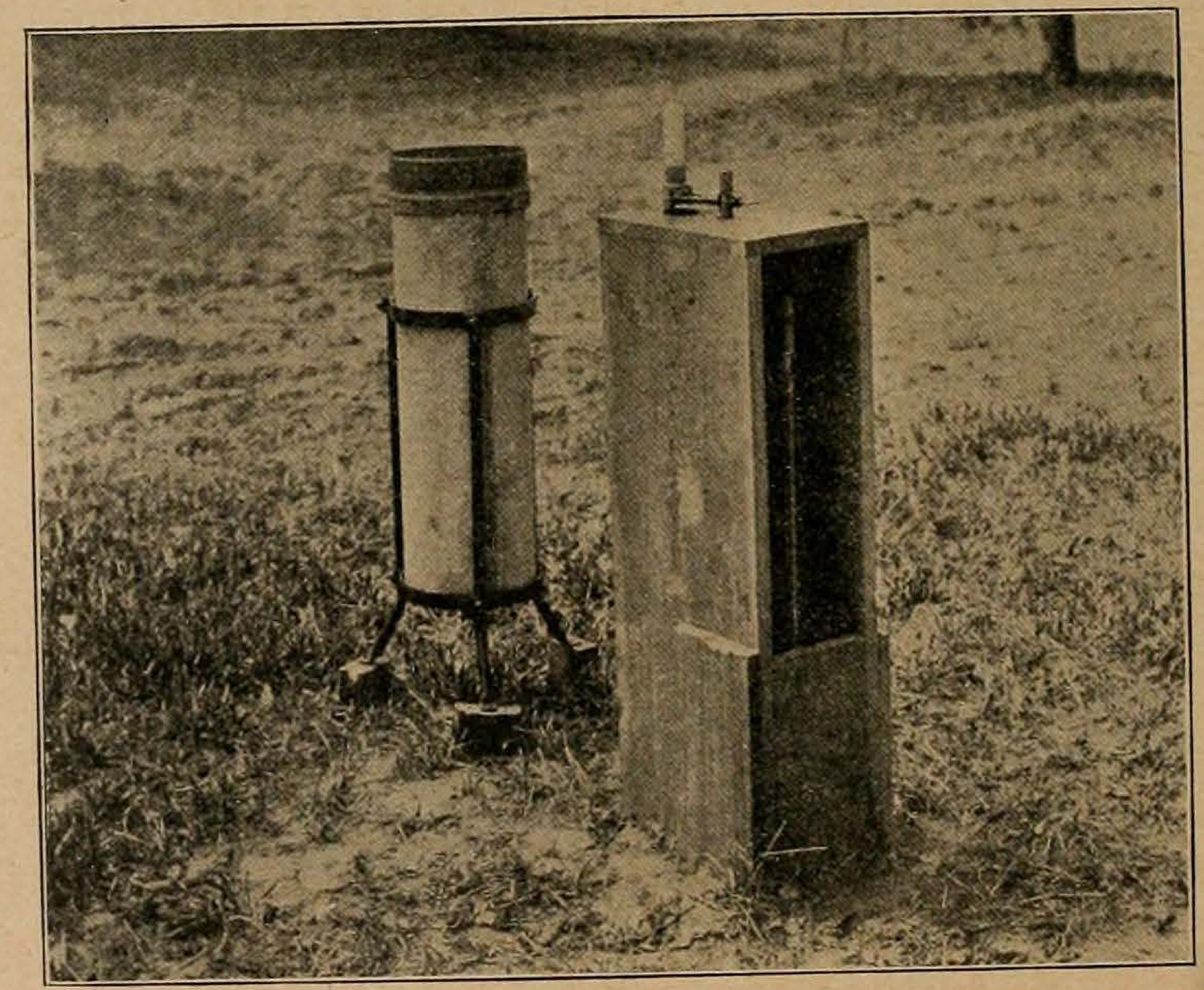

FIG. 8. Rain-gage (left), and atmometer (right) for determining rate of evaporation. 


\section{BAND-RECORD SERIES}

A number of trees were banded each year at each of the places where observations were made, both sprayed and unsprayed trees being usually included. The bands used were mostly two or three-ply burlap, about 4 inches wide. They were placed on the trees about June 1 and examined every day until the first larvæ were found; and after that every third day. A record was kept of the number of male and female larvæ collected. All the larvæ collected were placed either in pupal cages or emergence cages for further observation, and a partial record of pupation and a complete record of the emergence of moths were kept at Olney. In cases where both pupation and emergence were noted the pupal periods of those under observation were determined, and the data thus obtained were used in connection with studies on the influence of climatic conditions on the development of the pupa.

No attempt will be made to record all the details of each of these series, but the combined results of all of them have been used in our studies on climatic relations, life history, and seasonal history. Since a knowledge of the influence of climatic relations will assist in interpreting our life-history and seasonal-history data, a discussion of the climatic influence will be taken up first.

\section{Influence of Climatic Conditions on the EgG, Larva, AND PUPA OF the CODLING-Moth}

We ascertained by observations during 1915,1916 , and 1917 , that the incubation period varied from 4 to 15 days, the pupal period from 7 to 46 days, and the larval period from 18 to 45 days, and that the time of the appearance of the first brood of larvæ may vary as much as 19 days, and of the second brood 7 or 8 days in different seasons in the same locality, and that the variation in the time of the same events in various parts of the state in the same season may be as much as 20 or 25 days. These variations in length of the periods and dates of appearance of the broods are due principally to climatic conditions, and especially to temperature. It is the purpose of the present study to ascertain the relation of climatic conditions to the rate of development and to the time of the appearance of the broods and, as far as possible, to suggest methods by which these relations may be turned to practical account.

Under out-of-door conditions, temperature is the predominating factor in determining rate of development of the codling-moth. Evaporation and humidity have so little influence that they may be disregarded, except, perhaps, in seasons when there are prolonged periods of extremely high or low humidity.

The influence of temperature will be considered first. 
The fact that a certain degree of warmth is necessary for development of animal and vegetable life and that the rate of development is hastened by warm weather and retarded by cool weather has apparently always been known, but it is only within recent years that efforts have been made to ascertain the relative effects of different degrees of temperature.

\section{ZONES OF TEMPERATURE}

The entire range of temperature may be divided into six zones with respect to life and development, as shown below:

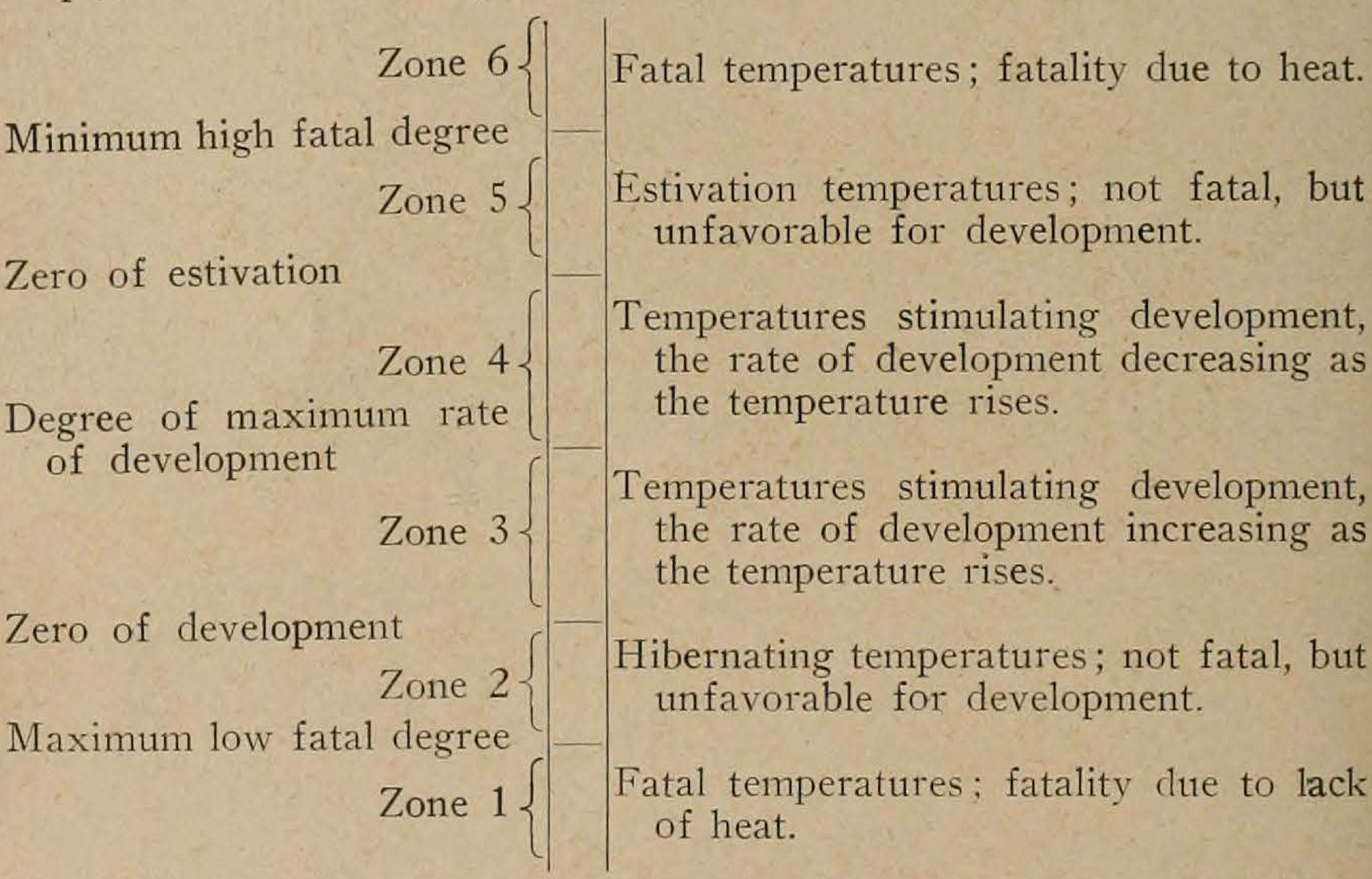

The limits of these zones differ for different species, for different stages of the same species, and for different phases of the same stage of the same species.

The limits of the zones of temperature of a species will be affected by the latitude to which it is acclimated, and it is possible that they will vary for individuals of the same species which are acclimated to different latitudes. Generally speaking, the limiting temperatures of the zones of species in low latitudes may be expected to be higher than of species in higher latitudes. The temperature at which an insect comes out of hibernation is in many cases quite different from that at which it becomes dormant in the latter part of the season, because heredity has an important influence in determining the time when insects begin the dormant period which lasts through the winter.

The temperatures on which these studies are based fall within zones 2,3 , and 4 , the purpose being to determine for the codling-moth the zero of development, or the point at which development begins in spring as 
the temperature rises; the degree at which development proceeds most rapidly ; and to find a temperature constant for each of the stages and for the whole period of development which will apply to all temperatures, and a method by which that constant can be determined.

Before proceeding very far it was discovered that the character of the climatic data obtained under field conditions was not well suited to studies of this kind, because the temperature varied constantly throughout the day, and consequently the deductions had to be made from averages of widely varying degrees of temperature. Early in the season the temperature during part of the day was in the zone of hibernating temperatures and during the remaining time in the zone of temperatures which stimulated development, and later in the season the temperatures during part of the day were in the zone in which the rate of development increased as the temperature rises and during the remainder of the day in the zone in which the rate of development decreased as the temperature rises. For scientific accuracy it is of interest to determine the relative effects of each degree of temperature, but from data of the kind here used this could not be undertaken.

It has already been pretty well established that the rate of development increases, approximately at least, as the temperature rises above the zero of development, until the temperature at which the rate of development is at its maximum is reached; and our studies have led us to conclude that as the temperature rises above the degree of the maximum rate of development the rate of development decreases directly as the temperature rises.

The day has been used as the unit of time, and the day-degree as the unit of accumulated temperatures; and it has been assumed that, within certain limits of temperature, the period expressed in days multiplied by the average daily day-degrees above the zero of development will approximate a constant, or at least that the average products of a large number of observations will approximate a constant.

GENERAL PROCEDURE IN THE STUDY OF TEMPERATURE RELATIONS

The first step taken was to prepare temperature tables from the thermographic records, showing the mean daily temperatures. In computing the mean daily temperatures, the day was regarded as beginning and ending at 5 o'clock p. m., that being the hour when observations for the day usually ended. All mean daily temperatures were ascertained by dividing the sums of the temperature readings for the even hours of the day by 12 . Mean temperatures above $50,52,85,86,87$, and 88 degrees were computed in the same way.

The second step was to tabulate in detail all the data relative to the length of the egg, pupal, and larval periods, tabulating the data for each generation and year separately and arranging the data in chronological order with respect to dates when the periods began and ended. 
The average mean daily temperatures and the length of the periods were determined for each observation. In all the groups of eggs, larvæ, and pupæ which began their periods on the same date there was a considerable variation in the recorded length of the period of individuals, due, no doubt, partly to the fact that individuals differ somewhat in respect to the amount of time required for them to develop even under identical conditions as to heat, light, moisture, and environment; and partly to errors of observation due for the most part, especially in case of eggs and pupæ, to the use of the day as the unit of time, on account of which the recorded period may, if the observations are made at the same hour each day, be almost a day longer or shorter than the true period, and, if the observations are not made at the same hour, the recorded period may be more than a day longer or shorter than the true period.

To avoid these variations the observations beginning on the same dates were thrown into groups, and the average period and average mean daily temperature were determined for each group. These smaller groups were then thrown into larger groups, using the mean daily temperature as the basis, all those passing through the period at an average mean daily temperature of 60 and 61 degrees or fraction thereof forming one group, and those passing through the period at temperatures of 62 and 63 degrees or fraction thereof forming another group, etc. The mean daily temperatures and the average length of the periods of these groups were then computed. All averages are weighted and all periods were averaged harmonically.

The detailed tables which it was necessary to prepare for the purpose of making the computations are voluminous and would not be of special interest. Only summaries made as above indicated will be given.

The reciprocal curve was used, with the mean daily temperature for the horizontal axis and the reciprocals of the periods for the vertical axis, to help locate approximately the zero of development and the degree of maximum rate of development; but this had to be supplemented by other methods on account of the displacement of points at the upper and lower ends of the line because of temperatures above the degree of the maximum rate of development and temperatures below the theoretical zero.

RELATION OF TEMPERATURE TO DEVELOPMENT OF THE EGG

The eggs used for observation were obtained from pairs of moths isolated on branches of trees in small wire cages. These cages were examined morning and evening. The day was used as the unit of time. Eggs found in the cages in the morning were recorded as having been laid the preceding day and those found in the afternoon were recorded as having been laid the same day. It is possible, therefore, that in some cases the recorded length of the period may be either almost a day longer or shorter than the actual period, but the averages of a large number of observations should approximate the true period very closely. 
table 1-Incubation Period at Olney, Illinors. Summary of Data on Relation of Temperature to DeVELopMent of Egg

\begin{tabular}{|c|c|c|c|c|c|c|c|c|c|}
\hline \multirow{2}{*}{$\begin{array}{l}\text { Temp. } \\
\text { range, } \\
\text { deg. Fah. }\end{array}$} & \multirow{2}{*}{$\begin{array}{l}\text { Number } \\
\text { of } \\
\text { observa- } \\
\text { tions }\end{array}$} & \multirow{2}{*}{$\begin{array}{l}\text { Harmonic } \\
\text { average } \\
\text { of periods } \\
\text { in days }\end{array}$} & \multirow{2}{*}{$\begin{array}{l}\text { Recipro- } \\
\text { cals of } \\
\text { periods }\end{array}$} & \multirow{2}{*}{$\begin{array}{l}\text { Mean } \\
\text { daily } \\
\text { tempera- } \\
\text { ture }\end{array}$} & \multicolumn{3}{|c|}{ Average daily day-degrees } & \multicolumn{2}{|c|}{ Total day-degrees } \\
\hline & & & & & $(50+)$ & $2(88+)$ & $\begin{array}{l}(50+)- \\
2(88+)\end{array}$ & $50+$ & $\begin{array}{l}(50+)- \\
2(88+)\end{array}$ \\
\hline $60-61$ & 46 & 14.00 & .0714 & 61.60 & 12.30 & & 12.30 & 172 & 172 \\
\hline $62-63$ & 229 & 12.67 & .0788 & 63.11 & 13.31 & & 13.31 & 170 & 170 \\
\hline $64-65$ & 622 & 10.66 & .0938 & 64.94 & 15.13 & & 15.13 & 161 & 161 \\
\hline $66-67$ & 305 & 9.35 & .1069 & 67.48 & 17.47 & & 17.47 & 163 & 163 \\
\hline $68-69$ & 595 & 8.67 & .1154 & 69.01 & 19.01 & & 19.01 & 165 & 165 \\
\hline $70-71$ & 515 & 7.72 & .1295 & 71.33 & 21.33 & , & 21.33 & 165 & 165 \\
\hline $72-73$ & 572 & 7.00 & .1425 & 73.12 & 23.12 & .01 & 23.11 & 162 & 162 \\
\hline $74-75$ & 335 & 6.60 & .1513 & 74.86 & 24.86 & .45 & 24.41 & 164 & 161 \\
\hline $76-77$ & 232 & 6.02 & .1663 & 77.43 & 27.43 & .96 & 26.47 & 165 & 159 \\
\hline $78-79$ & 249 & 5.95 & .1679 & 78.71 & 28.71 & .96 & 27.25 & 171 & 165 \\
\hline $80-81$ & 288 & 5.71 & .1750 & 80.14 & 30.14 & 1.93. & 28.21 & 172 & 161 \\
\hline $82-83$ & 141 & 5.52 & .1813 & 82.86 & 32.86 & 3.74 & 29.12 & 181 & 161 \\
\hline $84-85$ & 46 & 5.53 & .1807 & 84.00 & 34.00 & 4.66 & 29.34 & 188 & 162 \\
\hline $60-85$ & 4175 & 7.67 & .1304 & & 21.70 & & 21.33 & 166 & 163 \\
\hline
\end{tabular}


Table 1 is a final summary of the data on the relation of temperature to development of the egg. For the present we are concerned only with columns 4 and 5 -the reciprocals of the periods and the average mean daily temperatures.

In preparing Graph 1, convenient fractional parts were laid off on the vertical axis corresponding to the reciprocals of the periods and divisions were made on the horizontal axis corresponding to the mean daily temperatures, and the one was plotted against the other.

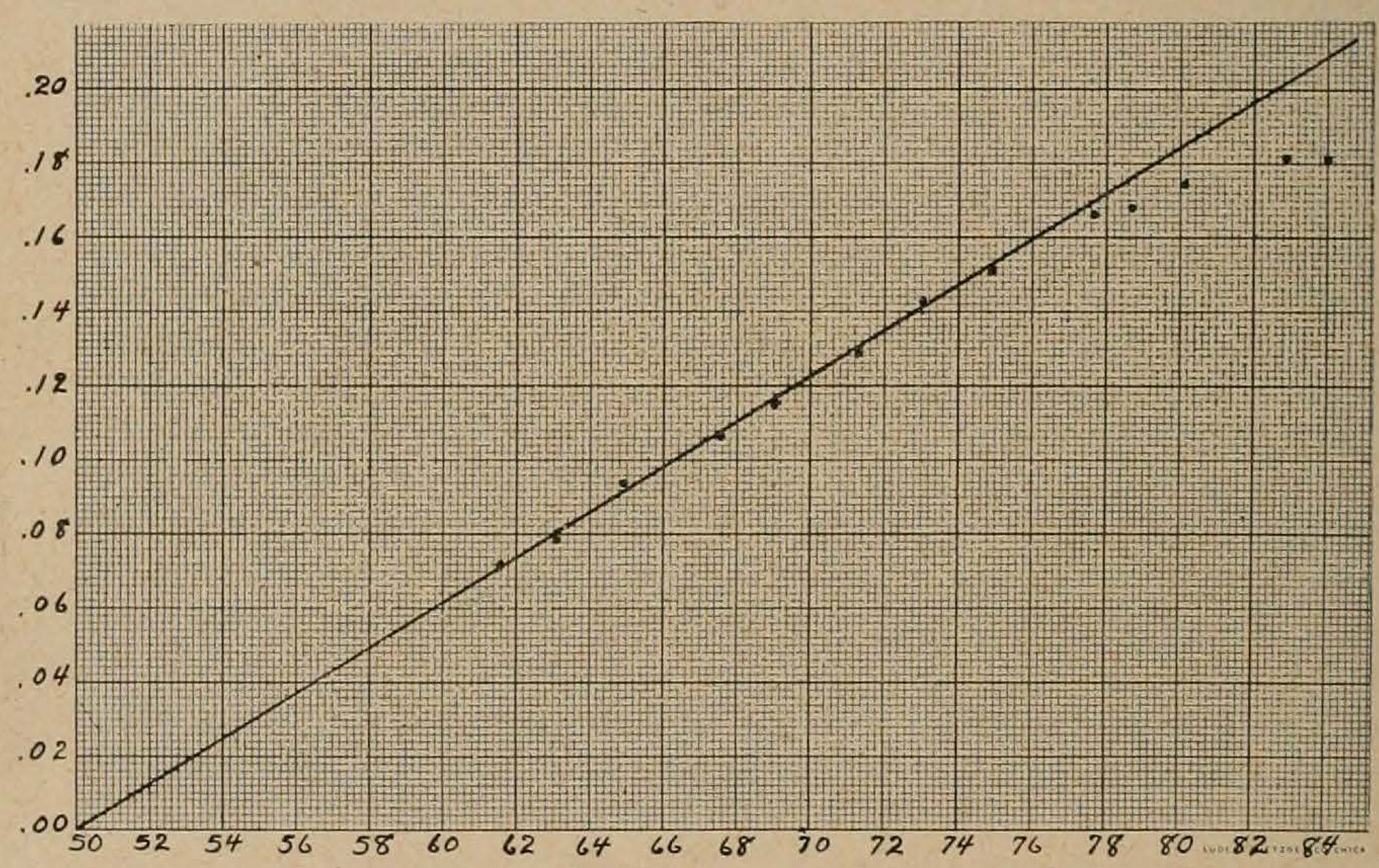

GRAPH 1. Incubation period. Reciprocals of the periods plotted against average mean daily temperatures.

The points below 76 degrees fall approximately in a straight line, those above 76 are displaced to the right because for part of the time during the periods the temperature was above the degree of maximum rate of development. The reciprocal curve determined by the points below 76 degrees crosses the temperature axis at 49.32 degrees. Since it is probable that some of these points used are somewhat displaced, the upper ones somewhat to the right on account of temperatures higher than the degree of maximum rate of development, the lower ones to the left on account of temperatures below the zero of development, this line is probably a little too flat and the true line will cross the temperature axis above $49.32^{\circ}$ rather than below it. Fifty degrees was taken as the approximate zero and was found to be nearly correct.

The average daily day-degrees above 50 degrees were computed for the different groups, and the results are recorded in column 6 of Table 1. Next, the averages in column 6 were multiplied by the length of the 
various periods (column 3 ) in order to get the total accumulation of daydegrees. These are recorded in column 9. If, in column 9, we disregard the accumulations for the first two groups at the top of the table, the accumulations are fairly constant till the 78-79 group is reached. Below this the accumulations rapidly increase, owing to the fact that the averages in column 6 contain some temperatures that are above the degree of the maximum rate of development and so retard development. It was necessary, therefore, to ascertain the degree of maximum rate of development, and to make the necessary corrections in the daily daydegrees column, so as to get only the effective day-degrees. In determining this point and making the correction, it was assumed that the products obtained by multiplying the average daily effective day-degrees by the periods would be nearly the same for all temperatures. It will not be necessary to give the details of the process by which the degree of maximum rate of development and the effects of temperatures above this point were determined. Suffice it to say that they were found by making guesses and testing the guesses out. It was found in this way that if 88 degrees were taken as the degree of maximum rate of development and twice* the number of day-degrees above this point (column 7) were subtracted from the average day-degrees above 50 degree, values for the daily effective day-degrees (column 8 ) were secured which when multiplied by the periods gave products that very nearly filled the conditions, giving about the same accumulation of effective day-degrees for the high temperatures as for the low.

By plotting the average daily day-degrees obtained in column 8 against the reciprocals of the periods, the points all fall somewhere near the established line (Graph 2). The average accumulation of effective day-degrees for the incubation period is, therefore, about 163.

No good reason can be assigned why the accumulations for the 60-61 and the 62-63 groups should be higher than the average unless it may have been that in assuming in 1915 that eggs found in the forenoon of one day were laid the previous evening, too long a period was assigned to many of them. The weather during the early part of 1915 was very cloudy and a larger per cent. of the eggs were laid during the daytime than in the other seasons.

* Twice the number of day-degrees above the degree of maximum rate of development is subtracted from the total day-degrees above the zero of development because one degree rise in temperature above the degree of maximum rate of development retards the rate of development at approximately the same rate as does one degree fall in temperature below this point. 


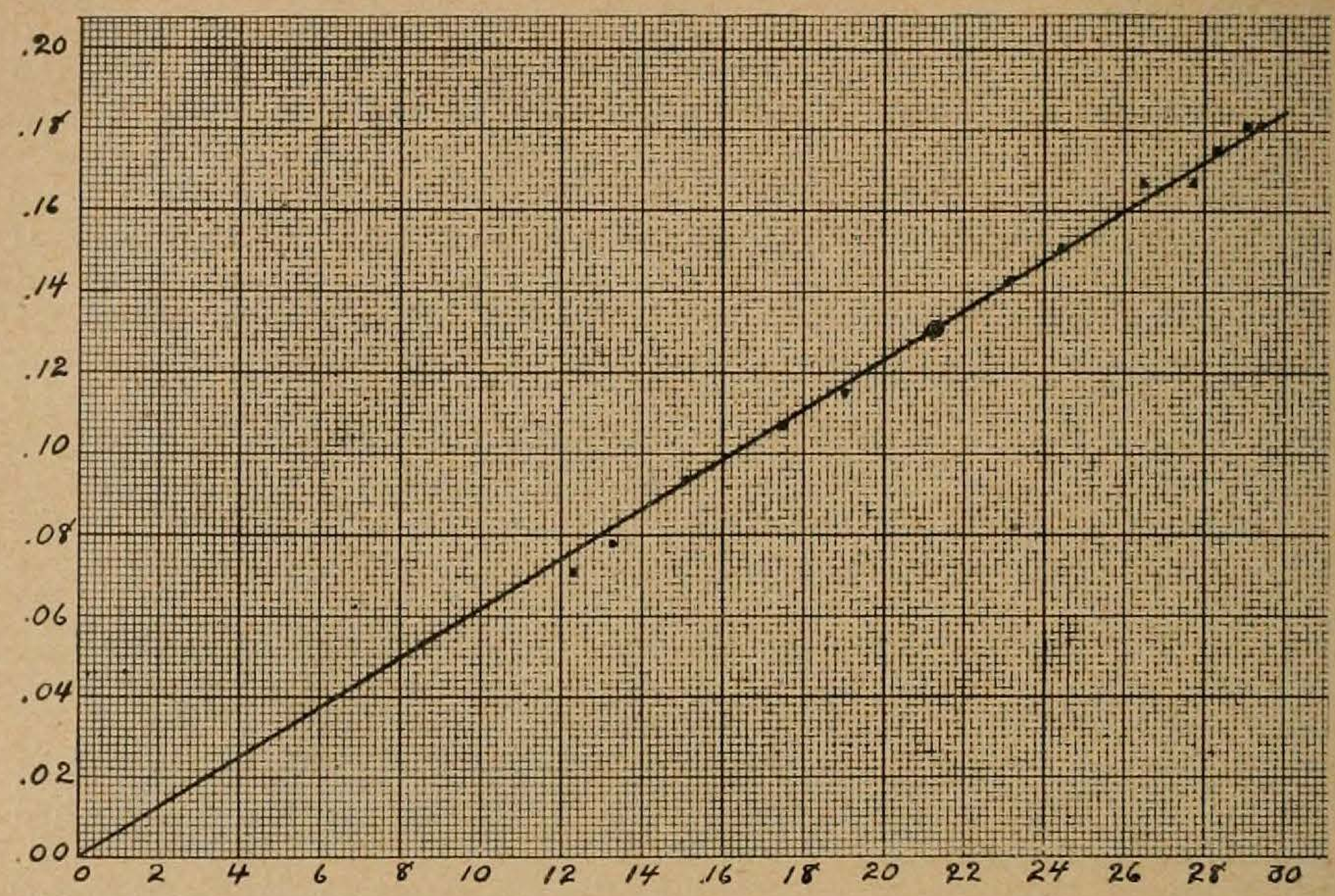

GRAPH 2. Incubation period. Reciprocals of the periods plotted against average daily effective day-degrees.

table 2-Distribution of the Eggs as to Number of the total Effective Daydegrees accumulated during the ObServed Period

\begin{tabular}{c|c}
\hline \hline Number of eggs & Range of effective day-degrees \\
\hline 35 & $119-128$ \\
29 & $129-138$ \\
1030 & $149-158$ \\
2163 & $159-168$ \\
745 & $169-178$ \\
171 & $179-188$ \\
2 & $189-198$
\end{tabular}


The variations in the total of effective day-degrees in the cases of individuals shown in the above table arises from several causes. Some of the extreme variations resulted, no doubt, from inaccuracies in observations; but for the most part they resulted from the use of the day as the unit, from which cause the recorded period may exceed or fall short of the true period by almost a day. Since in midsummer the daily accumulations may reach 27 effective day-degrees, the variations from this cause alone may be from 136 to 190 . The variations from this source are so large that they overshadow all the lesser ones and make it impossible to estimate the errors which probably arise from them. This source of error might have been avoided by using a smaller unit of time. There is also a difference in eggs as to the length of time required for the incubation period. Moisture, evaporation, and light also may have a slight influence on the rate of development.

It is probably true that variations arising from individual differences are comparatively slight and that the wide variations shown in the tables are due to the first two causes above mentioned. The extreme variations may therefore be regarded as of little significance and the average of the large number of observations may be relied upon as being approximately the true heat factor.

\section{RELATION OF TEMPERATURE TO DEVELOPMENT OF THE LARVA}

The conclusions arrived at are based upon observations made on 344 larvæ reared in apples on the tree and 214 larvæ reared in picked apples. It was found that larvæ reared in picked apples had a shorter period relative to the temperature than those reared in apples on the tree.

These data were first tabulated in detail and the periods and mean daily temperatures averaged for each of the observations, then for groups of larvæ which began the period on the same day, and lastly for groups arranged according to average mean daily temperatures. 'A summary of the results thus obtained from the observations on larvæ reared in apples on the tree is given in Table 3 . 
TABle 3-Larval Period. Summary of Data on Relation of Temperature to Development. Larvae reared in Apples on Tree.

\begin{tabular}{|c|c|c|c|c|c|c|c|c|c|}
\hline \multirow{2}{*}{$\begin{array}{l}\text { Temp. } \\
\text { range, } \\
\text { deg. Fah. }\end{array}$} & \multirow{2}{*}{$\begin{array}{c}\text { Number } \\
\text { of observa- } \\
\text { tions }\end{array}$} & \multirow{2}{*}{$\begin{array}{l}\text { Harmonic } \\
\text { average } \\
\text { of periods } \\
\text { in days }\end{array}$} & \multirow{2}{*}{$\begin{array}{l}\text { Recipro- } \\
\text { cals of } \\
\text { periods }\end{array}$} & \multirow{2}{*}{$\begin{array}{c}\text { Mean } \\
\text { daily tem- } \\
\text { perature }\end{array}$} & \multicolumn{3}{|c|}{ Daily day-degrees } & \multicolumn{2}{|c|}{ Total day-degrees } \\
\hline & & & & & $50+$ & $2(85+)$ & $\begin{array}{c}(50+)- \\
2(85+)\end{array}$ & $50+$ & $\begin{array}{l}(50+)- \\
2(85+)\end{array}$ \\
\hline $68-69$ & 32 & 35.57 & .028531 & 69.45 & 19.45 & .34 & 19.11 & 692 & 680 \\
\hline $70-71$ & 122 & 32.29 & .03091 & 70.88 & 20.88 & .30 & 20.58 & 674 & 665 \\
\hline $72-73$ & 45 & 29.20 & .03424 & 73.48 & 23.48 & .72 & 22.76 & 686 & 665 \\
\hline $74-75$ & 33 & 28.62 & .03495 & 74.53 & 24.53 & .74 & 23.79 & 702 & 681 \\
\hline $76-77$ & 14 & 27.14 & .03684 & 76.74 & 26.74 & 1.40 & 25.34 & 726 & 688 \\
\hline $78-79$ & 20 & 25.93 & .03855 & 79.39 & 29.39 & 3.62 & 25.77 & 762 & 668 \\
\hline $80-81$ & 78 & 26.33 & .03798 & 80.30 & 30.30 & 4.36 & 25.94 & 798 & 683 \\
\hline $68-81$ & 344 & 29.07 & .0338 & 74.31 & 24.31 & 1.56 & 22.75 & 719 & 673 \\
\hline
\end{tabular}


In Graph 3, fractional parts corresponding to the reciprocals of the periods (Table 3 ) were laid off on the vertical axis, and divisions were marked on the horizontal axis corresponding to the mean daily temperatures. Points were then plotted, using the reciprocals of the periods and the mean daily temperatures as the coordinates.

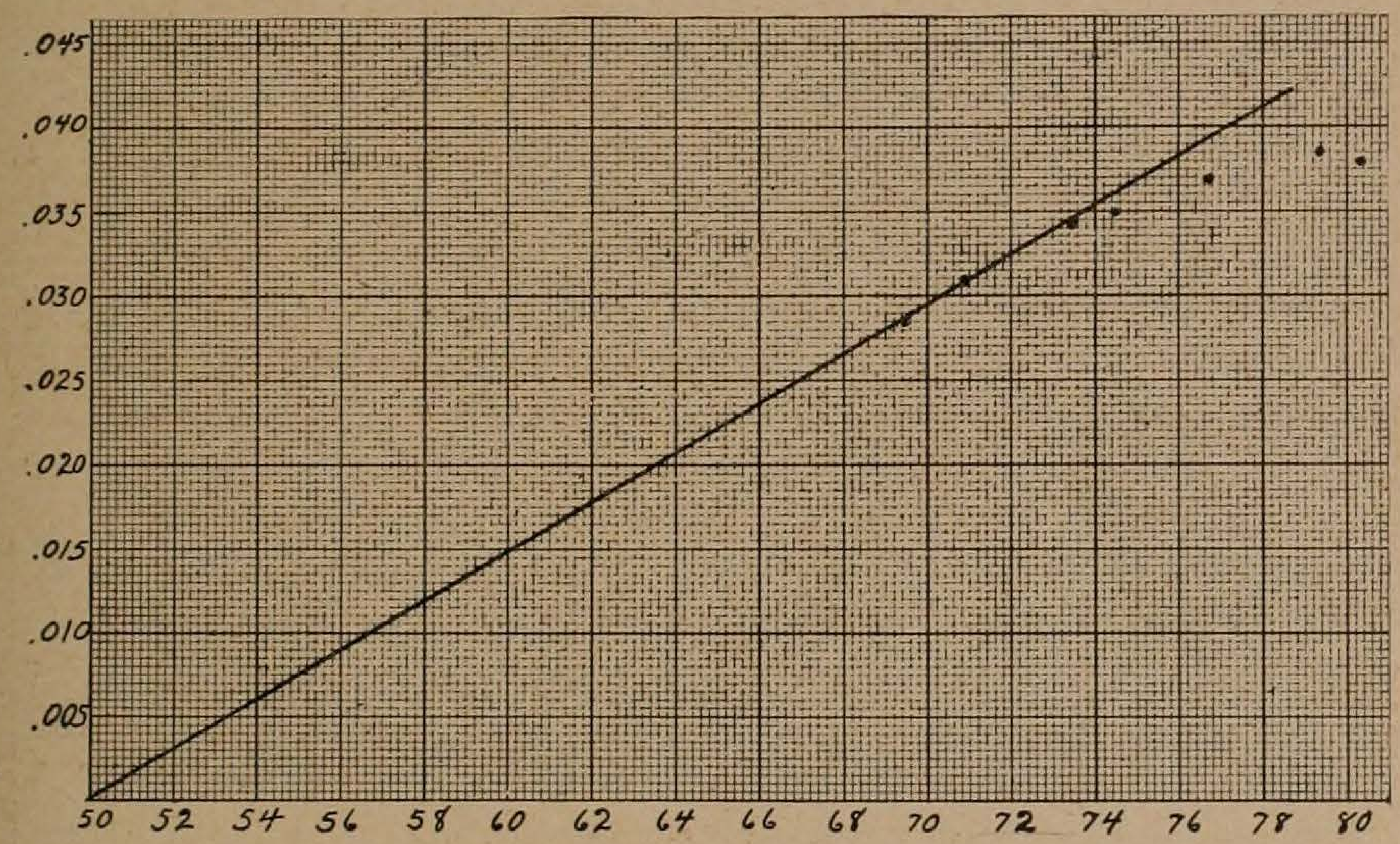

GRAPH 3. Larval period. Reciprocals of the periods plotted against mean daily temperatures. Larvæ reared in apples on tree.

These points do not lie in a straight line, and hence can not be used for the purpose of establishing the reciprocal curve accurately. The points do not lie in a straight line because in making the mean daily averages temperatures were used which are not effective or which retard development. The lower points are less affected by this cause than the other points because they represent temperatures that are intermediate between the zero of development and the degree of the maximum rate of development. A straight line determined by the two lowest points crosses the temperature axis at $52+$ degrees, a line determined by the three lowest points crosses the axis at $47+$. All points between 47 and 52 were tried out as to which best fulfilled the condition as the zero of development and 50 was selected as the one which did so most nearly.

The daily day-degrees above 50 degrees were computed and the results were entered in column 6 of Table 3 . The figures in this column multiplied by the number of days in the periods gave the total accumulations of day-degrees above 50 degrees, shown in column 9 of Table 3 . An examination of these results as well as the position of the upper points in Graph 3 leads us to conclude that the gradual increase in the accumulations and the deviation to the right of the points from the line at the 
higher temperatures were due to retarding temperatures or temperatures above the degree of maximum rate of development. To find this point guesses were made and tried out to test their correctness. It was found that by using 85 as the degree of the maximum rate of development, and subtracting twice the average day-degrees above 85 (column 7 ) from the day-degrees above 50 , we got a temperature factor (column 8 ) for each of the temperature groups which when multiplied by the period expressed in days gave the most nearly constant products (column 10) for the different groups, the average for all groups being 673 .

This average may be taken as the approximate average of accumulations of effective day-degrees for the larval period of larvæ reared in apples on the tree.

In Graph 4, the average daily effective day- degrees as recorded in column 8 of Table 3 were plotted against the reciprocals of the periods, with the result that the points in the upper part of the graph were brought nearly into line with the other points.

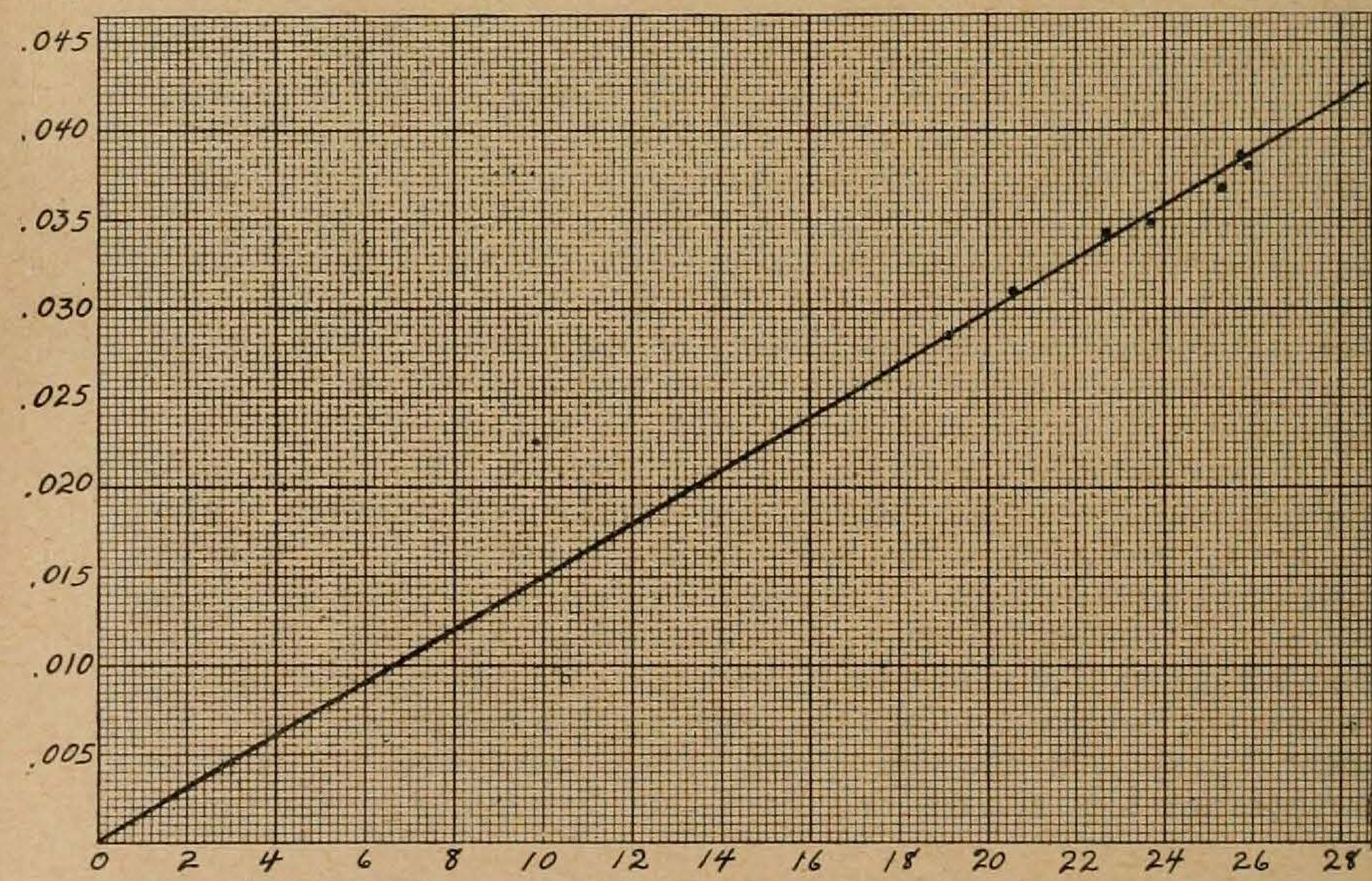

GRAPH 4. Larval period. Reciprocals of the periods plotted against mean daily effective day-degrees. Larvæ reared in apples on tree.

The data secured from observations made on 214 larvæ reared in picked apples were treated in the same manner as the data for larvæ reared in apples on the tree. A summary of the results is shown in Table 4. 
TABle 4-Larval Period. Summary of Data on Relation of Temperature to Development. Larvae reared in Picked Apples

\begin{tabular}{|c|c|c|c|c|c|c|c|c|c|}
\hline \multirow{2}{*}{$\begin{array}{l}\text { Temp. } \\
\text { range, } \\
\text { deg. Fah. }\end{array}$} & \multirow{2}{*}{$\begin{array}{c}\text { Number } \\
\text { of observa- } \\
\text { tions }\end{array}$} & \multirow{2}{*}{$\begin{array}{l}\text { Harmonic } \\
\text { average } \\
\text { of periods } \\
\text { in days }\end{array}$} & \multirow{2}{*}{$\begin{array}{l}\text { Recipro- } \\
\text { cals of } \\
\text { periods }\end{array}$} & \multirow{2}{*}{$\begin{array}{c}\text { Average } \\
\text { mean } \\
\text { daily tem- } \\
\text { perature }\end{array}$} & \multicolumn{3}{|c|}{ Average daily day-degrees } & \multicolumn{2}{|c|}{ Total day-degrees } \\
\hline & & & & & $50+$ & $2(85+)$ & $\frac{(50+)-}{2(85+)}$ & $50+$ & $\begin{array}{l}(50+)- \\
2(85+)\end{array}$ \\
\hline $66-67$ & 1 & 34.00 & .02941 & 67.90 & 17.90 & .20 & 17.70 & 608 & 601 \\
\hline $68-69$ & 15 & 33.11 & .03020 & 68.38 & 18.38 & .26 & 18.12 & 609 & 600 \\
\hline $70-71$ & 63 & 28.94 & .03456 & 71.25 & 21.25 & .46 & 20.79 & 615 & 602 \\
\hline $72-73$ & 43 & 26.68 & .03748 & 72.47 & 22.47 & .50 & 21.97 & 599 & 586 \\
\hline $74-75$ & 27 & 22.84 & .04378 & 74.82 & 24.82 & .84 & 23.98 & 567 & 548 \\
\hline $76-77$ & 25 & 23.86 & .04107 & 77.25 & 27.25 & 1.72 & 25.53 & 650 & 609 \\
\hline $78-79$ & 16 & 22.01 & .04543 & 79.08 & 29.08 & 3.00 & 26.08 & 640 & 573 \\
\hline $80-81$ & 24 & 21.54 & .04643 & 80.75 & 30.75 & 3.90 & 26.85 & 662 & 578 \\
\hline $66-81$ & 214 & 25.69 & .03892 & 74.08 & 24.08 & 1.22 & 22.86 & 619 & 587 \\
\hline
\end{tabular}


In computations for Table 4, 50 was taken as the zero of development and 85 as the degree of maximum rate of development. The results indicate that 52 degrees would have more nearly satisfied the conditions; but as the main interest in this table is that it gives an opportunity for comparing the rate of development of larvæ in apples on the tree and in picked apples, in order to have the same units of accumulation in both cases 50 was used as the zero. The average accumulation of effective day-degrees required to develop larvæ in picked apples was 587 , or 86 day-degrees less than for larvæ in apples on the tree (Table 3).

The variations in the length of the larval period are very great for individuals even when subjected to the same conditions. The following example will illustrate the extent of the variations. Of 19 larvæ hatched July 20,1916 , one pupated in 20 days, two in 23 days, three in 25 days, one in 27 days, three in 28 days, four in 29 days, three in 30 days, one in 31 days, and one in 34 days, giving a variation of 14 days in the length of the period. Individual variations are also shown by the varying number of effective day-degrees which accumulated during the larval period.

Tables 5 and 6 show the distribution of larvæ as to accumulated effective day-degrees.

TABLe 5-Distribution of Larvae reared in apples on the Treie with Reference to accumulated Effective Day-degrees during the Period

\begin{tabular}{c|c}
\hline Number of larvæ & Range of effective day-degrees \\
\hline 8 & $456-499$ \\
18 & $500-549$ \\
39 & $550-599$ \\
65 & $600-649$ \\
64 & $650-699$ \\
58 & $700-749$ \\
29 & $750-799$ \\
26 & $800-849$ \\
16 & $850-899$ \\
6 & $900-949$ \\
1 & $950-969$
\end{tabular}

The variations above 900 and below 500 are possibly due to errors in observations. The smaller variations are due in part to the use of the day as the period unit, and in part, no doubt, to the various temperatures 
to which larvæ in different parts of the tree are simultaneously subjected, to variations in the character of food, and to individual differences. How much of the variations are attributable to each of these causes can not be accurately determined from the data at hand.

The use of the day as the unit will take account of a variation of 27 day-degrees each way from the average. If we assume that variations below 500 and above 900 are due to errors of observation and make a correction of 27 on account of the use of the day as the unit, it still leaves a variation of from 527 to 873 to be attributed to other causes. Since there is no way of eliminating these causes in practical operations, we must expect under natural conditions that some larvæ will complete their period when the effective day-degrees have reached 527 and that others will not complete it until 873 effective day-degrees have accumulated, the average accumulation being 673 .

Table 6 shows the distribution of larvæ reared in picked apples as to total effective day-degrees required for their development.

TABLe 6-Distribution of Lakvae reared in Picked Apples with Reference to Accumulations of Eiffective Day-degrees during the Period

\begin{tabular}{c|c}
\hline \hline Number of larvæ & $\begin{array}{c}\text { Range of effective day-degrees } \\
3\end{array}$ \\
\hline 16 & $400-449$ \\
41 & $450-499$ \\
59 & $500-549$ \\
40 & $550-599$ \\
22 & $600-649$ \\
12 & $650-699$ \\
5 & $700-749$ \\
1 & $750-799$ \\
& $800-849$
\end{tabular}

Following the same course of reasoning with these data as with the preceding, the variations arising from factors which can not be eliminated under field conditions, range from 477 to 722 , the average being 587 .

These results are not very satisfactory for practical use. The averages are, no doubt, approximately correct; but the variations from the average are too great and the limits of the variations due to unpreventable causes are a little too uncertain to render the data entirely satisfactory for the purpose of forecasting life-history events. However, these are the nearest approximations to the actual accumulation of effective 
day-degrees for the larva and the variations therefrom that we have at the present time, and with similar data for the egg and pupa they will serve as a fairly satisfactory basis for forecasting events in the seasonal history of the codling-moth.

RELATION OF TEMPERATURE TO DEVELOPMENT OF THE PUPA

Observations were made on a total of 4348 pupæ of the codlingmoth. The data were tabulated, grouped, and averaged in the same manner as those for the other stages. A summary of the results is given in Table 7. 
table 7-Pupal Period, Olnex, Ilunois. Summary of Data on Relation of Temperature to Development

\begin{tabular}{|c|c|c|c|c|c|c|c|c|c|c|c|c|c|}
\hline \multirow{2}{*}{ 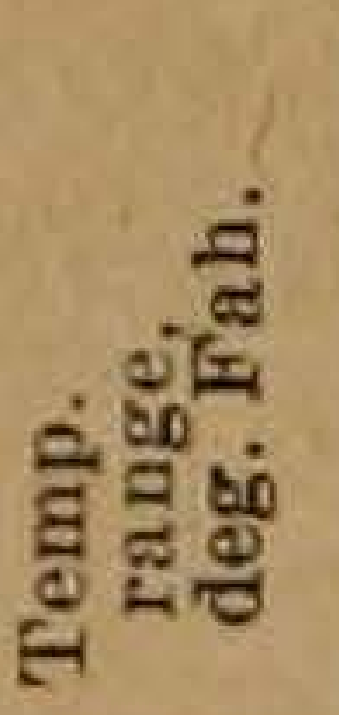 } & \multirow{2}{*}{ 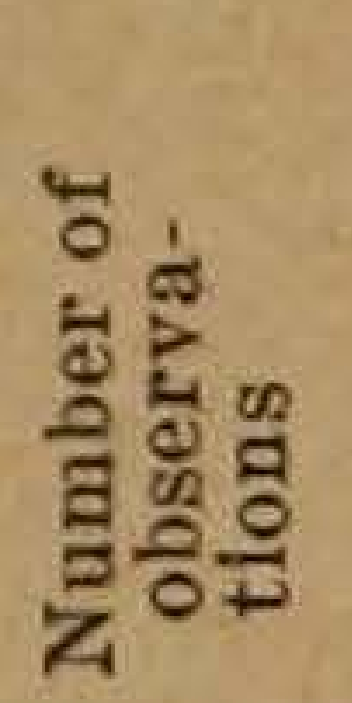 } & \multirow{2}{*}{ 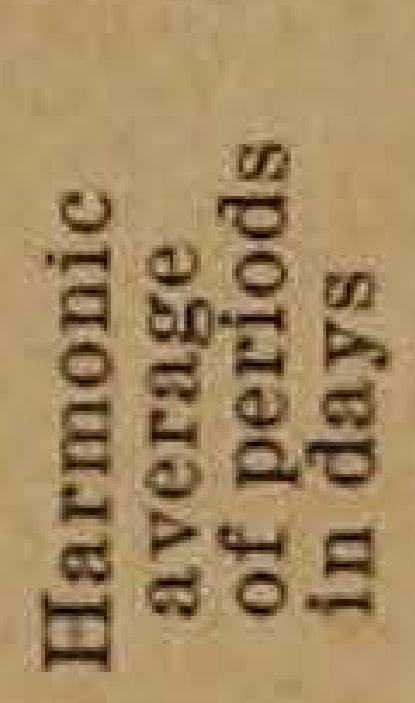 } & \multirow{2}{*}{ 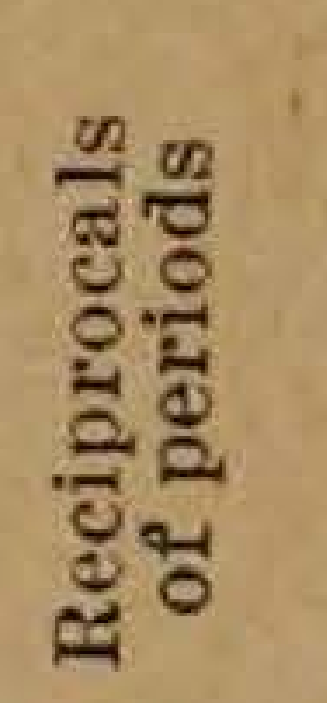 } & \multirow{2}{*}{ 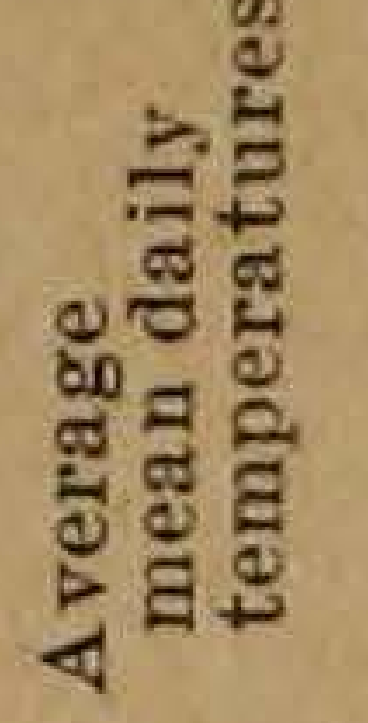 } & \multicolumn{5}{|c|}{ Average daily day-degrees } & \multicolumn{4}{|c|}{ Total day-degrees } \\
\hline & & & & & $50+$ & $52+$ & $\begin{array}{c}2(87 \\
+1\end{array}$ & $\begin{array}{l}(50+)- \\
2(87+)\end{array}$ & $\left(\begin{array}{l}52+1)- \\
2(87+)\end{array}\right.$ & $50+$ & $52+$ & $\left(\begin{array}{l}50+5)- \\
2(87+)\end{array}\right.$ & $\begin{array}{l}(52+)- \\
2(87+)\end{array}$ \\
\hline 52.53 & 2 & 45.50 & .0220 & 52.60 & 6.19 & 5.19 & & 6.19 & 5.19 & 281.6 & 236.0 & 281.6 & \\
\hline $54-55$ & 348 & 35.20 & .0284 & 55.70 & 7.85 & 6.80 & & 7.85 & 6.8 & 276.3 & 237.9 & 276.3 & \\
\hline $56-57$ & 976 & 34.01 & .0294 & 56.15 & 8.24 & 7.0 & & 8.24 & 7.08 & 280.2 & 240.8 & 280.2 & \\
\hline 58-59 & 243 & 27.77 & .0360 & 58.50 & 9.92 & 8.69 & & 9.92 & 8.6 & 275.5 & 241.3 & 275.5 & \\
\hline $60-61$ & 72 & 24.08 & .0416 & 61.13 & 11.75 & 10.39 & & 11.75 & 10.3 & 282 & 250.2 & 282.7 & \\
\hline $62-63$ & 148 & 22.21 & .0450 & 62.75 & 13.12 & 11.56 & & 13.12 & 11.5 & 291 & 256.7 & 291.4 & $256 . ?$ \\
\hline $64-65$ & 212 & 19.57 & .0511 & 65.02 & 15.46 & 13.6 & & 15.46 & 13.6 & 302.6 & 267.3 & 302.6 & 267. \\
\hline $66-67$ & 99 & 17.41 & .0574 & 66.59 & 16.90 & 15.15 & & 16.90 & & 294 & 263.8 & 294.2 & \\
\hline $68-69$ & 218 & 13.80 & .0724 & 69.28 & 19.27 & 17.3 & & 19.27 & 17.8 & 265 & 238.9 & 265 & \\
\hline 70-71 & 175 & 12.7 & $.07 \varepsilon$ & 70.79 & 20.7 & 18.7 & .04 & 20.75 & 18.75 & 264 & 238.6 & 263.5 & 23 \\
\hline $72-73$ & 221 & 11.5 & .086 & 73.11 & 23.7 & 21. & . 24 & 22 . & & $26 t$ & 242. & & \\
\hline 74-75 & 247 & 10.73 & .0932 & 74.92 & 24.92 & 22.92 & .42 & 24.50 & 22.5 & 267 & 245.9 & 262.9 & 241 \\
\hline $76-7$ & 517 & 10.02 & .09 & 76.77 & 26.7 & $24 .^{\prime}$ & .88 & 25. & 23. & 268 & 248.2 & 259.4 & 238 \\
\hline $78-79$ & 481 & 9.44 & .1059 & 79.14 & 29.13 & 27.1 & 1.63 & 27.50 & 25. & 275 & 256.1 & & \\
\hline $80-81$ & 133 & 9.43 & .1062 & 80.88 & 30.88 & 28.88 & 3.52 & 27.36 & 25. & 291.1 & 272.2 & 257.9 & \\
\hline $82-83$ & 256 & 9.24 & . 1082 & 82.68 & 32.68 & 30.68 & 4.12 & 28.56 & 26.56 & 302.0 & 283.5 & 263.9 & 245.4 \\
\hline $52-83$ & 3817 & 14.49 & .0690 & & 19.03 & 17.38 & .77 & 18.26 & 16.61 & 275.6 & 251.8 & 264.6 & $240 . ?$ \\
\hline
\end{tabular}

N. B.-The totals and averages of this table do not include the data given for $60^{\circ}-67^{\circ}$ inclusive. The pupæ noted at those temperatures being a part of the hibernating generation which pupated late, were disturbed by daily examinations which resulted in a prolongation of the normal pupal period. 
Graph 5 was prepared by plotting the reciprocals of the periods against the mean daily temperatures (columns 4 and 5, Table 7).

No three of the points in this graph appear to lie in a straight line. The points at the lower end of the line are too far to the left because in computing the mean daily temperatures temperatures below the zero of development were included, which prolongs the temperature axis too far to the left. Points at the upper end of the line are too far to the right because in computing the mean daily temperatures temperatures were included which were above the degree of the maximum rate of development and which, therefore, retarded development. The use of the mean daily degrees in this part of the graph prolonged the temperature axis too far to the right.

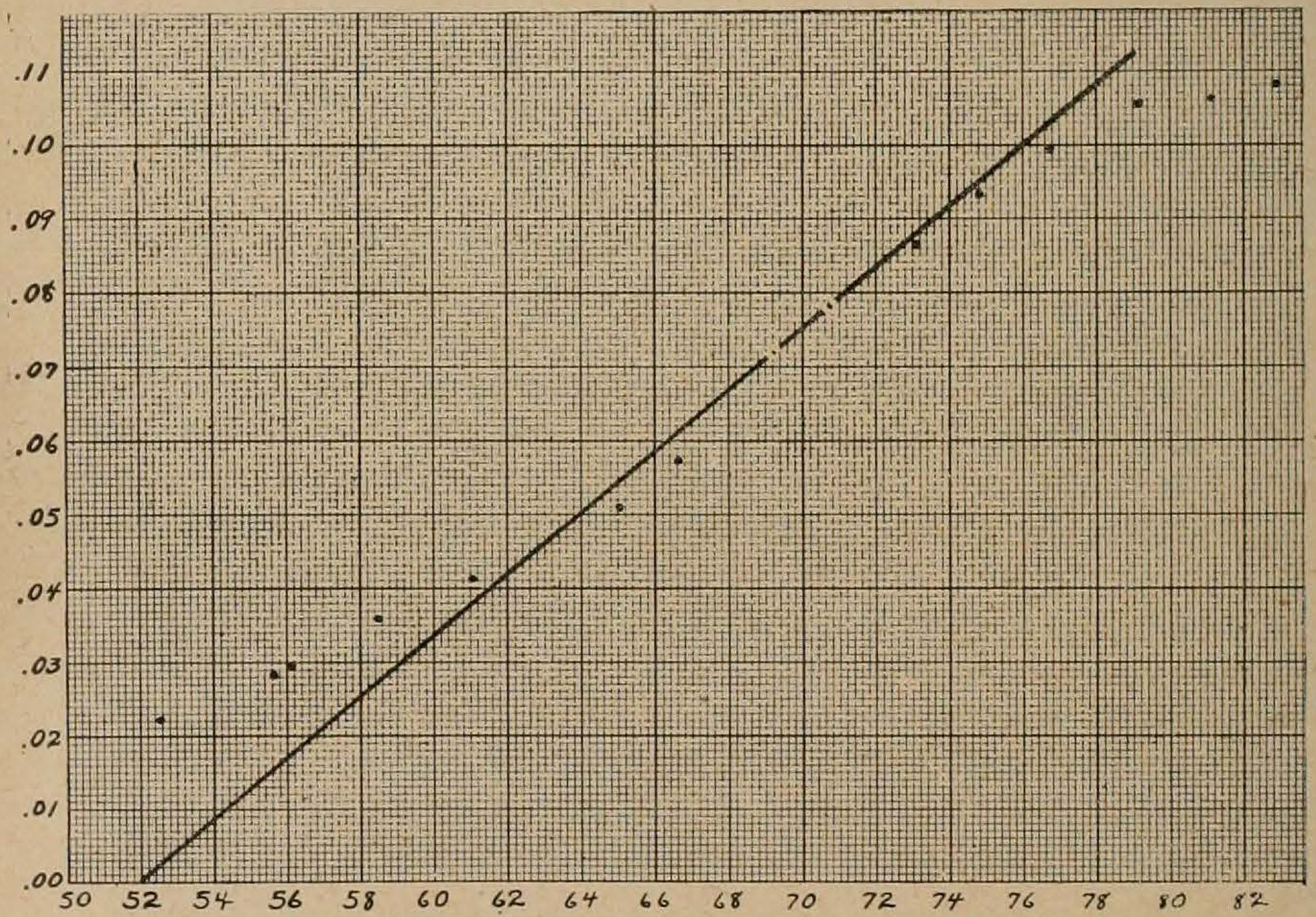

GrapH 5. Pupal period. Reciprocals of the periods plotted against average mean daily temperatures.

The points from 60 to 68 degrees are lower than they should be normally because these represent the portion of the hibernating generation which did not pupate until from two to six weeks after pupation began in the spring, and were consequently disturbed daily until they pupated by having their cocoons torn open to make observations possibie. The energy expended in repairing their cocoons daily seems to have devitalized them somewhat, on account of which a relatively longer period was required. Those which pupated last and hence were disturbed the most had the longest periods relative to the temperature. These points should, therefore, be disregarded. 
Because all the points seem to be displaced more or less on account of using ineffective and retarding temperatures in the temperature axis, they afford no means of determining the true reciprocal curve. The two points that are least affected are the middle points from 68 to 72 degrees. The line determined by these two points cuts the temperature axis at 51.92 degrees. By using $50,51,52$, and 53 degrees in turn as the possible zero of development it was found that 52 degrees more nearly satisfied the conditions than any other of the points.

To ascertain the degree of the maximum rate of development 86 , 87,88 , and 89 degrees were tried in turn. 87 most nearly satisfied the conditions.

The average daily day-degrees above 52 were computed and the results recorded in column 7 of Table 7 . Twice the average daily day-degrees above 87 degrees were computed and the results placed in column 8. By subtracting twice the average day-degrees above 87 degrees from the average daily day-degrees above 52 degrees we got the average daily effective day-degrees in column 10. By multiplying the daily effective day-degrees by the periods, we obtained the total accumulated effective day-degrees recorded in column 14. It will be noted that with the exception of that portion of the hibernating generation which pupated late, between 60 and 67 degrees inclusive, the total effective day-degrees are quite uniform for all temperatures. The retardation of the late portion of the hibernating generation due to the daily disturbance to which they were subjected is shown by the increased accumulation which was necessary to bring them through the stage. We concluded, therefore, that the effective day-degrees for the pupa might be computed by subtracting from the sum of the average daily day-degrees above 52 degrees, twice the average daily day-degrees above 87 degrees. The average of the effective daydegrees as thus determined is 240.7 .

Graph 6 was prepared by plotting the reciprocals of the periods against the average daily effective day-degrees given in columns 4 and 10 respectively of Table 7 . It will be noted that all the points with the exception of those which represent the late portion of the hibernating generation (10-16) lie nearly in a straight line, which shows that there is a quite constant relation between the average daily effective day-degrees as above determined, and the rate of development.

Observations on the pupal period were made at such a wide range of temperatures that the data exemplify very nicely the hyperbolic form of the curve of development formed by plotting the periods against the average daily effective day-degrees. Graph 7 has been prepared to show the different positions of the points occasioned by the use of different factors as coordinates and also the curve of development. 
Graph 7 combines Graphs 5 and 6, and also shows the curve of development. The circles show the position of the points when the reciprocals of the periods (Table 7 , column 4 ) are plotted against the average mean daily temperature (column 5); the dots show the position of the points when the reciprocals of the periods are plotted against the average day-degrees above $52^{\circ}$ (column 7 ); the dots with crosses show the position of the same points when the average daily effective

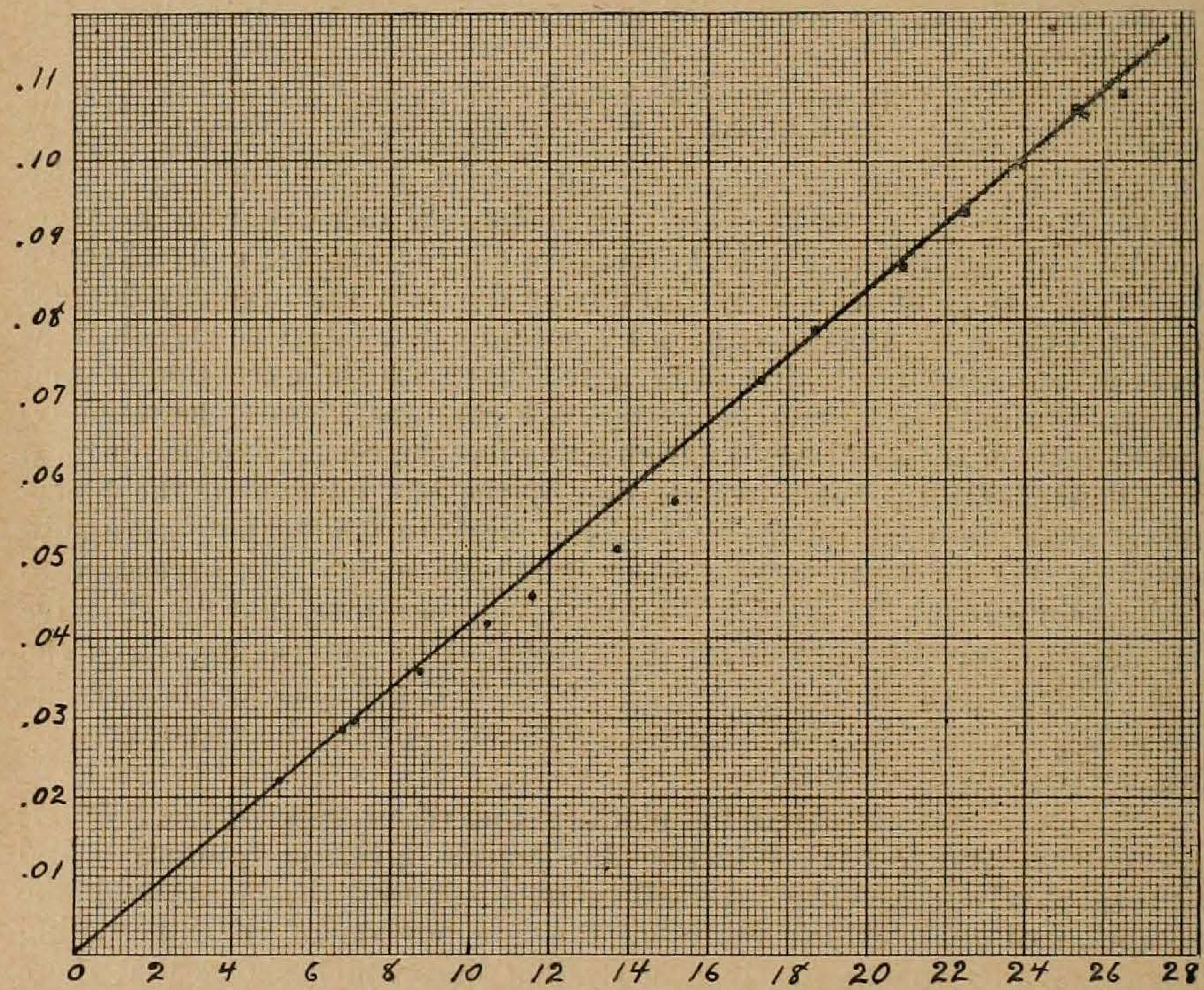

Graph 6. Pupal period. Reciprocals of the periods plotted against average daily effective day-degrees.

day-degrees after corrections were made for retarding temperatures above $87^{\circ}$ (column 10) are substituted for the average mean daily temperatures in the temperature axis; and the concentric circles show the position of the same points when the periods in days are plotted against the average daily effective day-degrees.

The latter gives an hyperbola of which the equation is $\mathrm{EP}=241$, in which $\mathrm{E}=$ average daily effective day-degrees and $\mathrm{P}=$ period in days. 


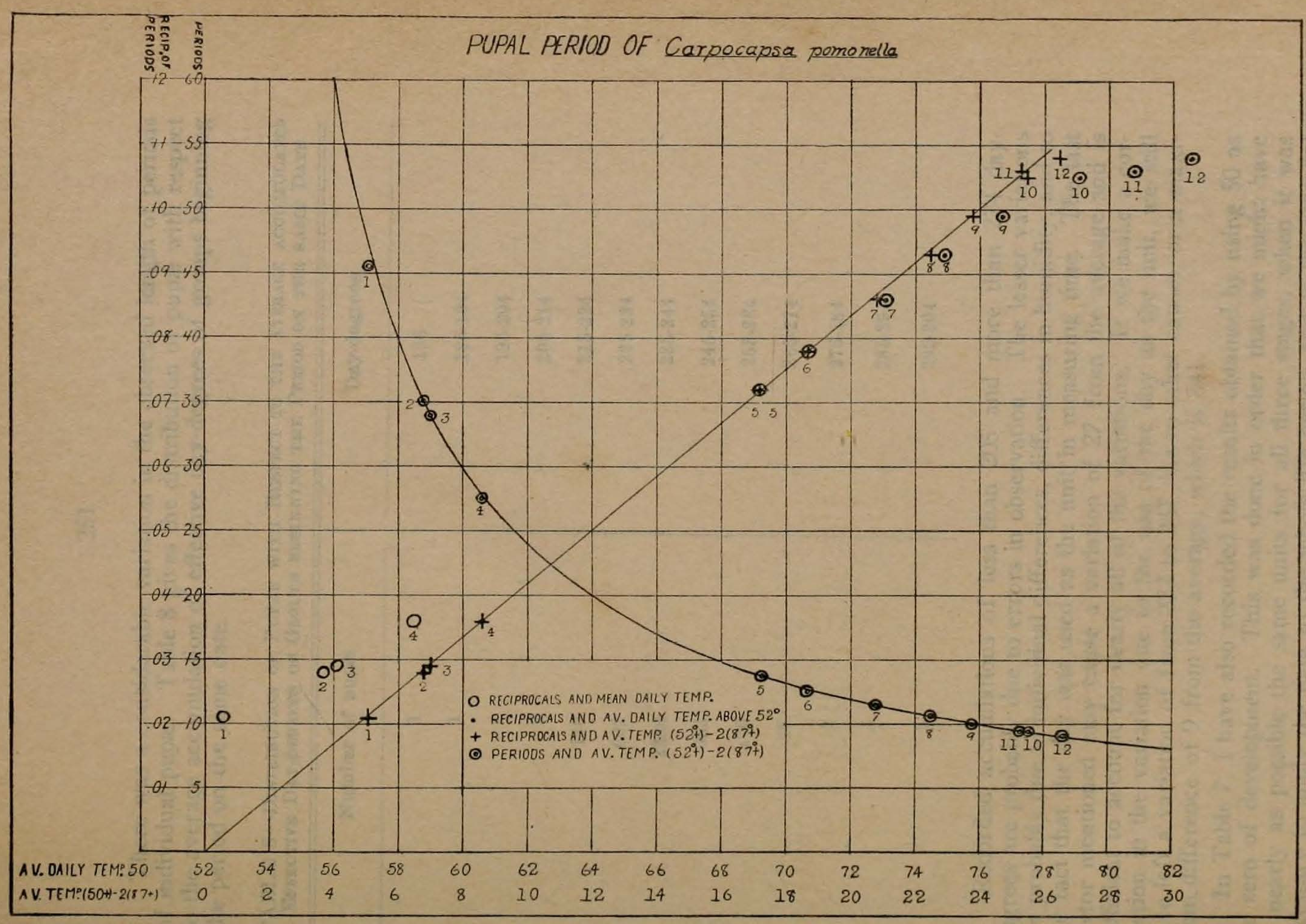

GrAPH 7. The time-temperature curve, reciprocal curve and different positions of points resulting from the use of different factors as coordinates, as indicated by the numerals under points. 


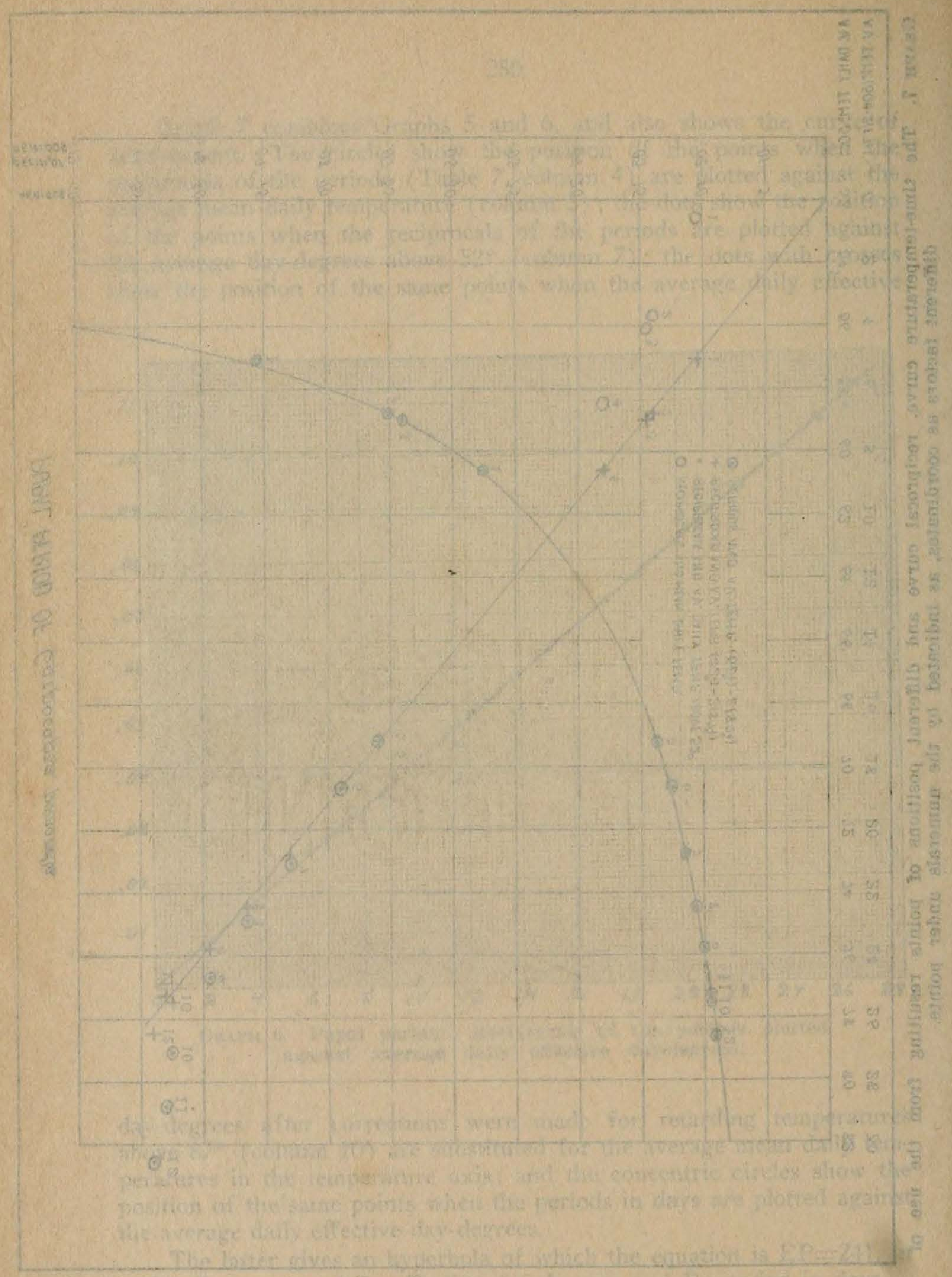

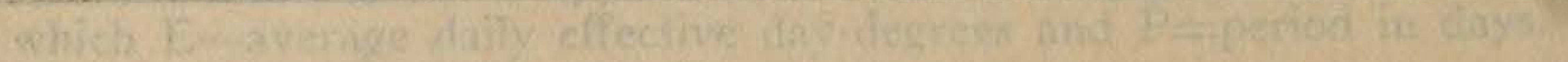


There was a considerable variation in the observed length of periods of individual pupæ. Table 8 gives the distribution of pupæ with respect to the average accumulation of effective day-degrees of groups beginning the period on the same date.

TABLE 8-Distribution of Pupae With Respect to the average accumulated Effective Day-degrees of Groups beginning the Period on the same Date

\begin{tabular}{c|c}
\hline \hline Number of pupæ & Day-degrees \\
1 & 166 \\
1 & $185-194$ \\
4 & $195-204$ \\
3 & $205-214$ \\
110 & $215-224$ \\
602 & $225-234$ \\
2238 & $235-244$ \\
353 & $245-254$ \\
170 & $255-264$ \\
18 & $265-274$ \\
7 & $275-284$ \\
7 & $285-294$ \\
2 & $295-304$
\end{tabular}

Recorded accumlations of less than 205 and more than 274 daydegrees are probably due to errors in observation. The lesser variations are probably due to individual differences, differences in humidity, and to the fact that the day was used as the unit in measuring time. The last factor mentioned may cause a variation of 27 from the average and is sufficient to account for nearly all of the variations. If we make a correction in the variation due to the use of the day as the unit, we still have left a variation of from 232 to 247 due to other causes, or a maximum difference of 9 from the average, which is 241 .

In Table 7 , I have also recorded the results obtained by using 50 as the zero of development. This was done in order that we might have as nearly as possible the same units for all three stages, when it was necessary to apply the data to the whole life period, and to the seasonal history. The average accumulation of effective day-degrees using 50 as 
the zero of development is 265 , and the variation due to causes other than the use of the day as the unit gives 256 as the minimum and 274 as the maximum.

\section{CONCLUSIONS FROM THE FOREGOING DISCUSSION}

1. There is a fairly constant relation between temperature and the rate of development of different stages of the codling-moth.

2. Only temperatures above the zero of development are effective.

3. The rate of development increases as the temperature rises above the zero of development until a temperature is reached at which the maximum rate of development takes place.

4. For the first few degrees, at least, above the degree of the maximum rate of development the rate of development decreases approximately as the temperature rises above this point.

5. The average daily effective day-degrees are found by subtracting from the average daily day-degrees above the zero of development twice the average daily day-degrees above the degree of maximum rate of development.

6. The product obtained by multiplying the average daily effective day-degrees by the period, expressed in days, or other convenient unit, is fairly constant for all temperatures.

7. Variations in the total accumulation of day-degrees recorded for individuals from the average accumulation, may result from the following causes:-1. Use of too large a unit of time. 2. Differences in humidity. 3. Individual differences. 4. Errors in observation. 5. Slight differences of temperature between the individual under observation and the recording instrument, due to difference of locality. 6. Abnormal environment and treatment of the individual.

8. The zero of development for the egg stage is about 50 degrees $\mathrm{F}$. and the degree of the maximum rate of development is about 88 . The equation of the curve of development is $\mathrm{PE}=163$, in which $\mathrm{P}=$ period in days, and $\mathrm{E}==$ average daily effective day-degrees.

9. The zero of development for the larva is about 50 degrees and the degree of the maximum rate of development is about 85 degrees. The equation of the curve of development for larvæ reared in apples on the tree is $\mathrm{PE}=673$; that for larvæ reared in picked applies is $\mathrm{PE}=587$.

10. The zero of development for the pupa is about 52 and the degree of maximum rate of development is about 87 degrees. The equation of the curve of development is $\mathrm{PE}=241$. For practical purposes it will be more convenient to use 50 as the zero of development for the pupa when applying the data to the whole life cycle, and the error that will result from so doing will amount to only a fraction of a day in the length of the period. The equation when 50 degrees is used, is $\mathrm{PE}=265$. 


\begin{tabular}{|c|c|c|c|c|c|c|c|c|c|c|c|c|c|c|c|c|c|c|c|c|c|}
\hline & & & & & & & & & & & & Life & perio & ds in 0 & ays. & & & tal effec & Ive day & legrees. & \\
\hline Sex. & $\begin{array}{l}\text { Egg } \\
\text { lald. }\end{array}$ & & $\begin{array}{c}\text { Larva } \\
\text { hatched. }\end{array}$ & & $\begin{array}{l}\text { Larv8 } \\
\text { left } \\
\text { apple }\end{array}$ & & $\begin{array}{l}\text { Larva } \\
\text { pupate }\end{array}$ & & $\begin{array}{l}\text { Adult } \\
\text { emerge }\end{array}$ & & 茏 & 염 & 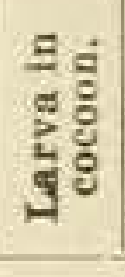 & 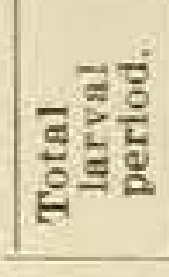 & $\stackrel{\vec{a}}{\vec{a}}$ & 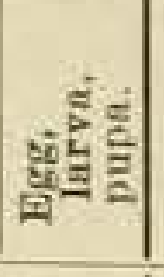 & 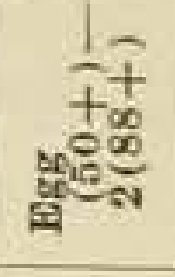 & 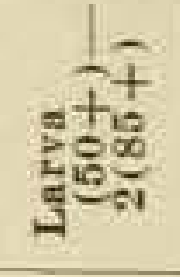 & 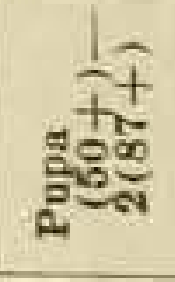 & 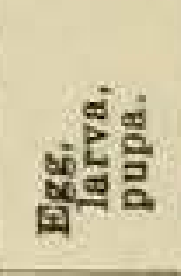 & 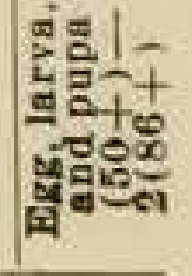 \\
\hline F: & May 2 & 21 & June & 3 & June & & June 3 & & July 1 & 13 & 13 & 26 & 1 & 27 & 13 & 53 & 174 & 559 & 255 & 988 & 989 \\
\hline M. & & 21 & & 3 & & & July & 1 & & 14 & 13 & 26 & 2 & 28 & 13 & 54 & 174 & 580 & 264 & 1018 & 1017 \\
\hline F. & $\ddot{~}$ & & ". & & & & & & "̈ & & 13 & & & & & 54 & 174 & & & 1018 & 1017 \\
\hline M. & . & 21 & 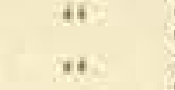 & 3 & . & & & 3 & " & 16 & 13 & & & 30 & 13 & 56 & 174 & 621 & 284 & 1079 & 1078 \\
\hline F. &.. & 21 & .. & 3. & ". & $\begin{array}{l}29 \\
30\end{array}$ & $"$. & 4 & & 16 & 13 & 26 & 5 & 31 & 12 & 56 & 174 & 636 & 269 & 1079 & 1078 \\
\hline M. & .. & 21 & $"$. & 3 & ." & 30 & & 5 & * & 16 & 13 & 27 & 5 & 32 & 11 & 56 & 174 & 648 & 258 & 1080 & 1078 \\
\hline F. & & 21 & ". & 3 & July & 1 & " & 7 & ". & 18 & 13 & 28 & 6 & 34 & 11 & 58 & 174 & 682 & 284 & 1140 & 1138 \\
\hline F. & " & 21 & "* & 2 & & 5 & * & 10 & ". & 18 & 12 & 33 & 5 & 38 & 8 & 58 & 157 & 759 & 225 & 1141 & 1138 \\
\hline F. & “. & 21 & " & 3 & & & ". & 9 & $\cdot$ & 19 & 13 & & & 36 & 10 & 59 & 174 & 719 & 274 & 1167 & 1165 \\
\hline F. & & 21 & " & 3 & June: & & " & 9 & & 19 & 13 & 27 & 9 & 36 & 10 & 59 & 174 & 719 & 274 & 1167 & 1165 \\
\hline F. & & & $"$ & 3 & July & & ". & 12 & " & & 13 & 32 & 6 & 39 & 10 & & 174 & 787 & 271 & 1232 & 1229 \\
\hline M. & & 22 & " & 3 & June: & & ". & 4 & ". & 17 & 12 & 27 & 4 & 31 & 13 & & 159 & 636 & 299 & 1094 & 1093 \\
\hline F. & 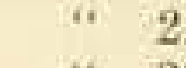 & 22 & $\ddot{*}$ & 3 & July & 7 & " & 12 & $\ddot{*}$ & 21 & 12 & 34 & 5 & 39 & 9 & & 159 & 787 & 252 & 1198 & 1195 \\
\hline M. & & & $"$ & 4 & & & $"$ & 9 & " & 19 & 12 & & & 35 & 10 & 5 & 169 & 698 & 274 & 1141 & 1139 \\
\hline M. & “. & 23 & " & 4 & ". & 4 & 4 & 10 & ". & 20 & 12 & 30 & 6 & 36 & 10 & 5 & 169 & 720 & 279 & 1168 & 1165 \\
\hline F. & " & 23 & ." & 4 & * & & “ & 14 & * & 25 & 12 & 36 & 4 & 40 & 11 & 6 & 169 & 821 & 277 & 1267 & 1267 \\
\hline F. & $*$ & 31 & $"$ & 9 & June & & " & 4 & ت & 15 & 9 & 21 & 4 & 25 & 11 & 45 & 159 & 525 & 238 & 922 & 921 \\
\hline M. & " & 31 & ". & 0 & & & " & 7 & " & 17 & 10 & & & 27 & 10 & 47 & 174 & 556 & 253 & 983 & 982 \\
\hline M. & " & 31 & ". & 0 & & & ". & 8 & "* & 18 & 10 & & & 28 & 10 & & 174 & 575 & 265 & 1014 & 1011 \\
\hline M. & " & 31 & "* & 8 & & & “ & 10 & " & 19 & 8 & & & 32 & 9 & 49 & 148 & 641 & 252 & 1041 & 1034 \\
\hline F. & " & 31 & " & 7 & July & 7 & $"$ & 12 & " & 20 & 7 & 30 & 5 & 35 & 8 & 50 & 139 & 695 & 23 & 1067 & 1065 \\
\hline M. & " & 31 & $"$ & 8 & & & $\therefore$ & 10 & " & 20 & 8 & & & 32 & 10 & 5 & 148 & 641 & 27 s & 1068 & 1065 \\
\hline F. & ". & 31 & " & 9 & & & “ & 10 & " & 20 & 9 & & & 31 & 10 & 5 & 159 & 630 & 27 & 1068 & 1065 \\
\hline M & ". & 31 & ". & 7 & ". & 6 & " & 11 & $"$ & 21 & 7 & 29 & 5 & 34 & 18 & & 139 & 671 & 27 & 1086 & 1084 \\
\hline M. & " & 31 & " & 9 & ". & 6 & ". & 11 & ". & 21 & 9 & 27 & 5 & 32 & 10 & 51 & 159 & 652 & 276 & 1087 & 1084 \\
\hline F. & ". & 31 & ". & 9 & . & 7 & " & 12 & " & 22 & 9 & 28 & 5 & 33 & 10 & 5 & 159 & 676 & 27 & 1106 & 1103 \\
\hline M. & 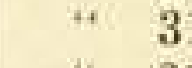 & 31 & “. & 9 & & & " & 13 & $"$ & 23 & 9 & & & 34 & 10 & & 159 & 703 & 264 & 1126 & 1123 \\
\hline F. & " & 31 & .. & 9 & & & " & 13 & ." & 23 & 9 & & & 34 & 10 & & 159 & 70 & 26 & 1126 & 1123 \\
\hline M. & . & 31 & " & 9 & ". & 13 & ". & 16 & *. & 27 & 9 & 34 & 3 & 37 & 11 & & 159 & 78 & 26 & 1217 & 1220 \\
\hline F. & " & 31 & ". & 8 & ". & 12 & " & 16 & . & 28 & 8 & 34 & 4 & 38 & 12 & & 149 & 80 & 2 ! & 1246 & 1244 \\
\hline M. & " 43 & 31 & " & 9 & " & 14 & *. & 18 & " & 29 & 9 & 35 & 4 & 39 & 1 & 5 & 159 & 84 & 26 . & 1271 & 1275 \\
\hline F. & June & 1 & ." & 1 & June : & 29 & "* & 3 & " & 15 & 10 & 18 & 4 & 22 & 12 & 44 & 182 & 47 & 25 & 908 & 906 \\
\hline F. & " & 1 & ". & & July & 3 & " & 8 & “" & 18 & 9 & 23 & 5 & 28 & 10 & 3 & 159 & 57 & $2 f$ & 999 & 996 \\
\hline M. & $"$ & 1 & “ & 1 & & 3 & " & 8 & " & 18 & 10 & 22 & 5 & 27 & 10 & & 182 & 55 & 26 & 999 & 996 \\
\hline M. & " & 1 & " & 1 & & & " & 11 & ". & 21 & 10 & & & 30 & 1. & & 182 & 61 & 27 & 1072 & 1069 \\
\hline F. & " & 1 & " & 1 & & & " & 14 & " & 25 & 10 & & & 33 & 11 & 5 & 182 & 69 & 27 & 1153 & 115 \\
\hline F. & " & 1 & " & 1 & & & " & 14 & " $\quad 2$ & 25 & 10 & & & 33 & 11 & & 182 & 69 & $2 ?$ & 1153 & 115 \\
\hline F. & " & 1 & " 1 & & " & 17 & " & 21 & Aug. & 1 & 9 & 37 & 4 & 41 & 12 & 6 & 159 & 90 & 28 & 1345 & 1345 \\
\hline M. & Tune & 2 & June 1 & & & & July & 4 & July 1 & 16 & 9 & & & 23 & $1:$ & 4 & 166 & 48 & 26 & 923 & 921 \\
\hline M. & " & 2 & " 1 & & " & 6 & . $\quad 1$ & 12 & $\pi$ & 22 & 9 & 25 & 6 & 31 & 10 & 5 & 166 & 63 & 27 & 1075 & 1072 \\
\hline F. & " & 2 & " & & & & " & 12 & $"$ & 22 & 9 & & & 31 & 10 & & 166 & 63 & 27 & 1075 & 1072 \\
\hline M. & “ & 2 & " & & " & 8 & $"$ & 13 & " & 23 & 9 & 27 & 5 & 32 & 11 & & 166 & 68 & & 1093 & 1093 \\
\hline F. & “ & 2 & " & & ". & 12 & ". & 14 & " & 25 & 9 & 31 & 2 & 33 & 11 & & 166 & 69 & 27 & 1137 & 1136 \\
\hline F. & “ & 2 & ". & & " & 14 & " & 16 & $"$ & 29 & 9 & 33 & 2 & 35 & 1 & & 16 & 75 & 32 & 1243 & 1244 \\
\hline F. & “ & 2 & " & 1 & ". & 14 & ." & 18 & " & 29 & 9 & 33 & 4 & 37 & 11 & & $16 t$ & 81 & 26 . & 1240 & 1244 \\
\hline E. & " & 2 & ". & & " & 14 & " & 19 & " & 30 & 9 & 33 & 5 & 38 & 1 & & 16 & $8:$ & & 1270 & $127:$ \\
\hline E. & ". & 2 & ". & 1 & & & “. & 20 & " 3 & 31 & 9 & & & 39 & 11 & 5 & 166 & $8 t$ & & 90 & 130 \\
\hline M. & " & 2 & " & 1 & " & 24 & " & 28 & Aug. & & 9 & 43 & 4 & 47 & 1 & & 16 & 10 & & 1473 & $147 \mathrm{~s}$ \\
\hline M. & “ & 3 & 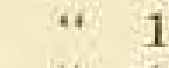 & & & & June 3 & 30 & July 1 & 13 & 9 & & & 18 & 1 & 4 & 174 & & & 815 & 81 \\
\hline F. & $*$ & 3 & " & 2 & “ & 2 & July & 7 & * 1 & 17 & 9 & 20 & 5 & 25 & 1 & 4 & 174 & 50 & & 936 & 93 \\
\hline M. & ". & 3 & " & & & & $"$ & 9 & " & 19 & 9 & & & 25 & 1 & & 174 & 5 & 27 & 994 & 99 \\
\hline M. & . & 3 & * & 2 & “ & 5 & "* & 10 & " & 20 & 9 & 23 & 5 & 28 & 10 & 4 & 174 & 56 & 27 & 1021 & 101 \\
\hline F. & " & 3 & “ & & “ & 11 & " & & “ & 24 & 9 & 29 & 3 & & & & 17 & 6 & 25 & 109 & 109 \\
\hline F. & ". & 3 & " & 1 & " & 8 & " & 14 & & 25 & 8 & 27 & 6 & 33 & 1 & & 14 & $6 !$ & 27 & $11:$ & 111 \\
\hline F. & " & 3 & " & 2 & “ & 10 & “ & & “ & 26 & 9 & $2 \varepsilon$ & 4 & & & & 17 & & 27 & 1121 & 111 \\
\hline F. & " & 3 & “ & 2 & " & 12 & " & 16 & ". & 28 & 9 & 30 & 3 & 33 & 1 & & 17 & & 24 & 11 & 111 \\
\hline F. & ." & 3 & " & 1 & " & 12 & ". & 1 & " & : & 8 & 31 & 3 & 34 & & & 14 & 7 . & 24 & 1118 & 1119 \\
\hline F. & ". & 3 & 1 & 2 & & & ". & 16 & ". & 27 & 9 & & & 34 & & & 17 & 7 & 26 & 11 & \\
\hline F. & ". & 3 & 1 & & & & “ & 1 & ". & 28 & 9 & & & 34 & 1 & & 17 & 72 & 29 & 119 & \\
\hline F. & . & 3 & 1 & 2 & ". & 16 & " & 1 ? & ". & 29 & 9 & 34 & 3 & $3 ?$ & 1 & & 17 & & & 12 & \\
\hline M. & ". & 3 & 1 & 1 & “ & 14 & " & 1 : & “. & 30 & 8 & 35 & 4 & 3 & 1 & & 14 & & & 12 & \\
\hline F. & $*$ & 3 & 1 & 3 & & & ". & 19 & $\begin{array}{llll} & . & 3\end{array}$ & 31 & 10 & & & $3 t$ & 12 & & 20 & & & 12 & \\
\hline F. & ". & 3 & “ & & $"$ & 18 & ". & 22 & Aug. & 1 & 9 & 36 & 4 & 4 & 9 & & 17 & 8 & & 131 & 13. \\
\hline F. & " & 3 & “ & 2 & " & 19 & ". & 25 & $"$ & 2 & 9 & 37 & 4 & 41 & 9 & & 17 & 8 & 26 & 7 & \\
\hline F. & " & 3 & “ & 2 & & & " 2 & 23 & “. & 2 & 9 & & & 41 & 9 & & 17. & 8 & 26 & 1337 & \\
\hline F & ." & 3 & " & & & & " & 25 & " & 4 & 9 & & & 43 & 10 & & 17 & 941 & 263 & $13 ?$ & 1381 \\
\hline F. & “ & 3 & ". & 2 & " & 21 & " & 26 & " & 6 & 9 & 39 & 5 & 44 & 11 & 64 & 174 & 967 & 272 & 1413 & \\
\hline F. & * & 4 & " & & & & & & July 1 & 8 & 8 & & & & & 44 & 153 & & & 942 & \\
\hline AI. & " & 4 & " & 2 & " & 8 & " 1 & 12 & " $\quad 2$ & & 8 & 2 & 4 & 3 & 9 & 4 & 153 & 61 & 25 & 1018 & \\
\hline F. & " & 5 & " & & " & 8 & " 1 & 18 & “ 2 & 2 & 8 & 25 & 4 & 29 & 16 & 47 & 15 & 5 & 27 & 1015 & \\
\hline M & " & 5 & ." & 3 & & & $"$ & & “ & 4 & 8 & & & 31 & 10 & 4 & 15 ? & 64 & 25 & 1054 & 10 \\
\hline $\mathrm{F}$ & ." & 5 & " 1 & 5 & & & " 1 & 16 & " 2 & 6 & 8 & & & 33 & 10 & 51 & 157 & 70 & 242 & 1099 & 109 \\
\hline $\mathrm{F}$ & " & 5 & $"$ & 4 & "̈ & 12 & $"$ & 18 & $\begin{array}{ll}\text { " } 2 & 2\end{array}$ & 7 & 9 & 28 & 6 & 34 & 9 & 52 & 179 & 73 & 208 & 1124 & 1119 \\
\hline M. & . & 5 & " 1 & 4 & “ & 26 & " 2 & 28 & Aug. & & 9 & 42 & 2 & 44 & 12 & 55 & 179 & 970 & 290 & 1439 & 1438 \\
\hline
\end{tabular}

SUMMARY OF TABLE 9

\begin{tabular}{|c|c|c|c|c|c|c|c|c|c|c|c|c|c|c|}
\hline \multirow{3}{*}{ Sex. } & \multicolumn{2}{|c|}{ Egg. } & \multicolumn{2}{|c|}{ Larva in fruit. } & \multicolumn{2}{|c|}{ Larva in cocoon. } & \multicolumn{2}{|c|}{ Larva. } & \multicolumn{2}{|c|}{ Pupa. } & \multicolumn{4}{|c|}{ Egg, larva, and pupa. } \\
\hline & \multirow{2}{*}{ No. } & \multirow{2}{*}{$\begin{array}{c}\text { Perlod } \\
\text { in } \\
\text { days. }\end{array}$} & \multirow{2}{*}{ No, } & \multirow{2}{*}{$\begin{array}{c}\text { Period } \\
\text { In } \\
\text { deys. }\end{array}$} & \multirow[b]{2}{*}{ No. } & \multirow{2}{*}{$\begin{array}{c}\text { Period } \\
\text { in } \\
\text { days. }\end{array}$} & \multirow[b]{2}{*}{ No. } & \multirow{2}{*}{$\begin{array}{c}\text { Period } \\
\text { In } \\
\text { days. }\end{array}$} & \multirow[b]{2}{*}{ No. } & \multirow{2}{*}{$\begin{array}{c}\text { Period } \\
\text { in } \\
\text { days. }\end{array}$} & \multirow[b]{2}{*}{ No. } & \multirow{2}{*}{$\begin{array}{c}\text { Period } \\
\text { in } \\
\text { days. }\end{array}$} & \multicolumn{2}{|c|}{$\begin{array}{l}\text { Total effective } \\
\text { day-degrees. }\end{array}$} \\
\hline & & & & & & & & & & & & & $\begin{array}{l}\text { Sum for } \\
\text { stnges. }\end{array}$ & $\frac{(50+)}{2(86+)}$ \\
\hline
\end{tabular}


TABLE 10-Life-history Data on First Generation, Olaney, 1916. Lafvae reared in Apples on Tree

\begin{tabular}{|c|c|c|c|c|c|c|c|c|c|c|c|c|c|c|c|c|c|c|c|}
\hline \multirow[b]{2}{*}{ ڤั } & \multirow[b]{2}{*}{$\begin{array}{l}\text { Egg } \\
\text { Iaid. }\end{array}$} & \multirow[b]{2}{*}{$\begin{array}{c}\text { Larva } \\
\text { hatehed. }\end{array}$} & \multirow[b]{2}{*}{$\begin{array}{l}\text { Larva } \\
\text { left } \\
\text { apple. }\end{array}$} & \multirow[b]{2}{*}{$\begin{array}{c}\text { Larva } \\
\text { pupated. } \\
-\end{array}$} & \multirow[b]{2}{*}{$\begin{array}{c}\text { Adult } \\
\text { emerged. }\end{array}$} & \multirow[b]{2}{*}{$\begin{array}{l}\text { Adult } \\
\text { died. }\end{array}$} & \multicolumn{8}{|c|}{ Length of life periods. } & \multicolumn{5}{|c|}{ Total effective day-degrees. } \\
\hline & & & & & & & 题 & 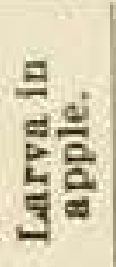 & $\begin{array}{l}\text { 틍 } \\
\text { 형 } \\
\text { 용 } \\
\text { 3्र }\end{array}$ & हैं & 范 & 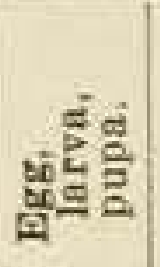 & 붕혐 & छี & 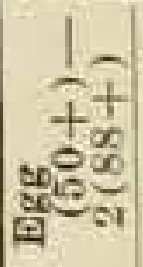 & 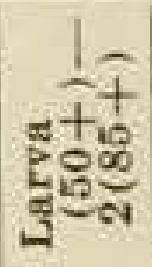 & $\frac{1}{15}$ & 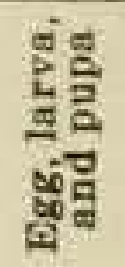 & 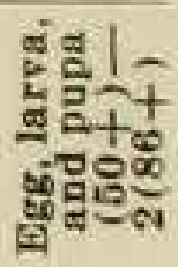 \\
\hline
\end{tabular}

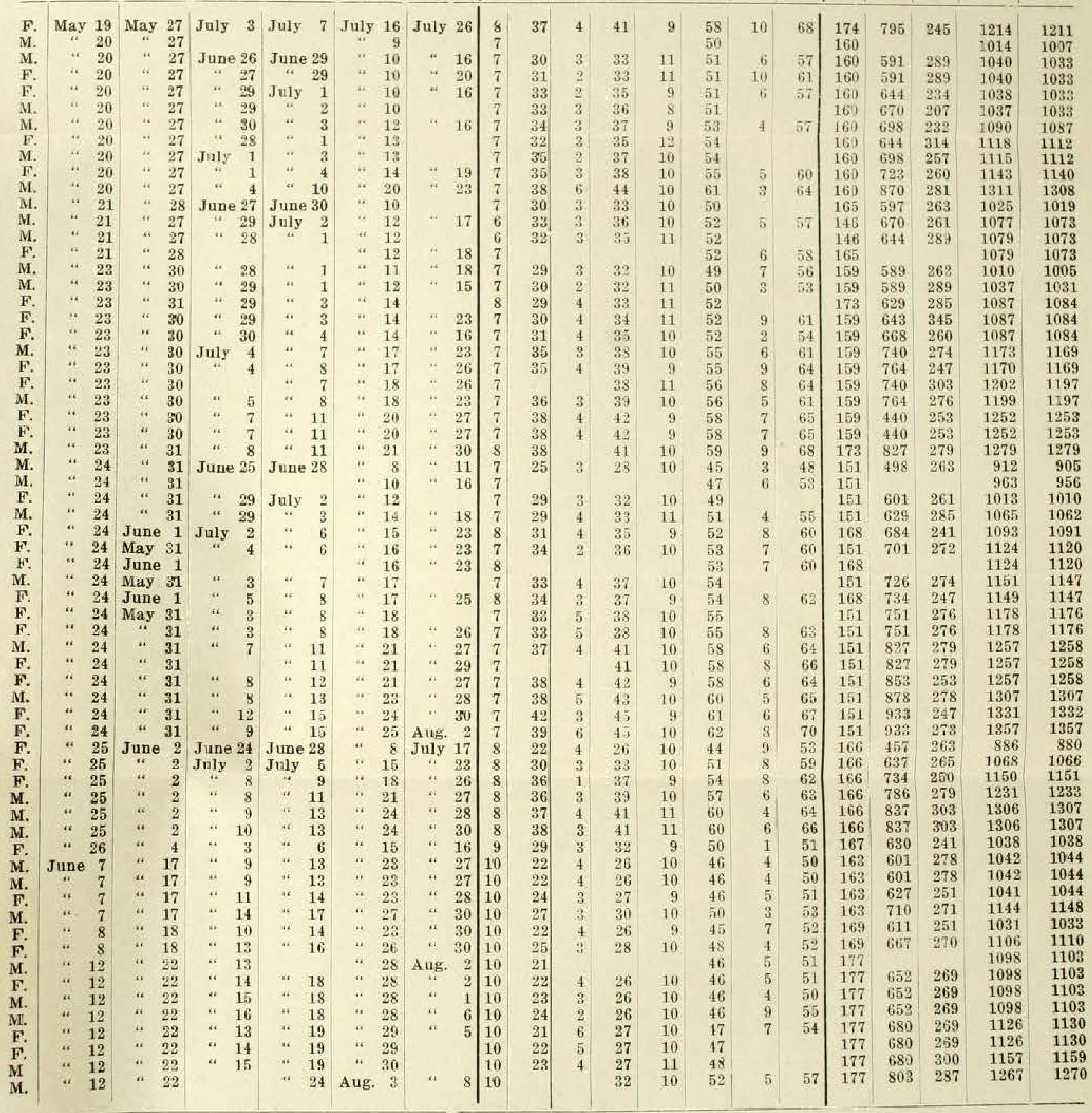

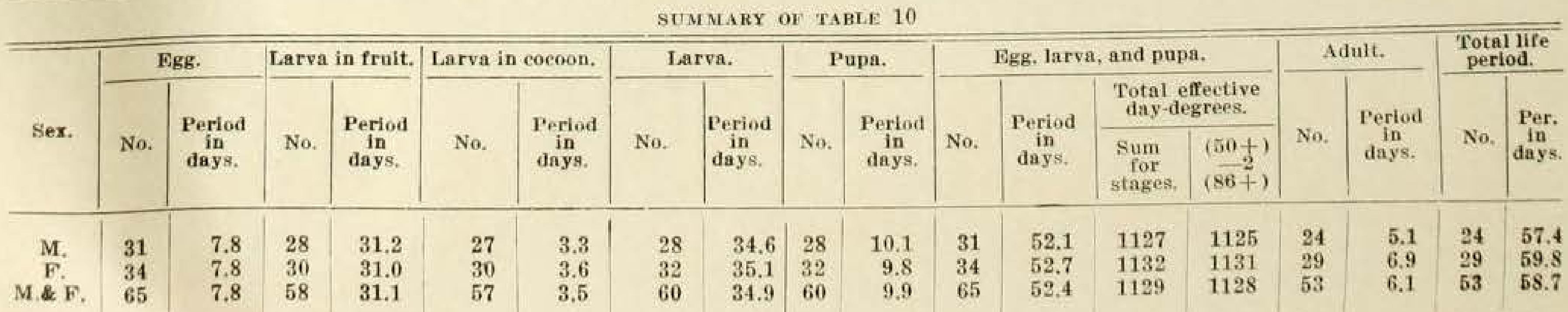




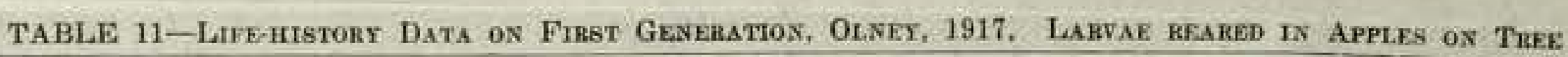

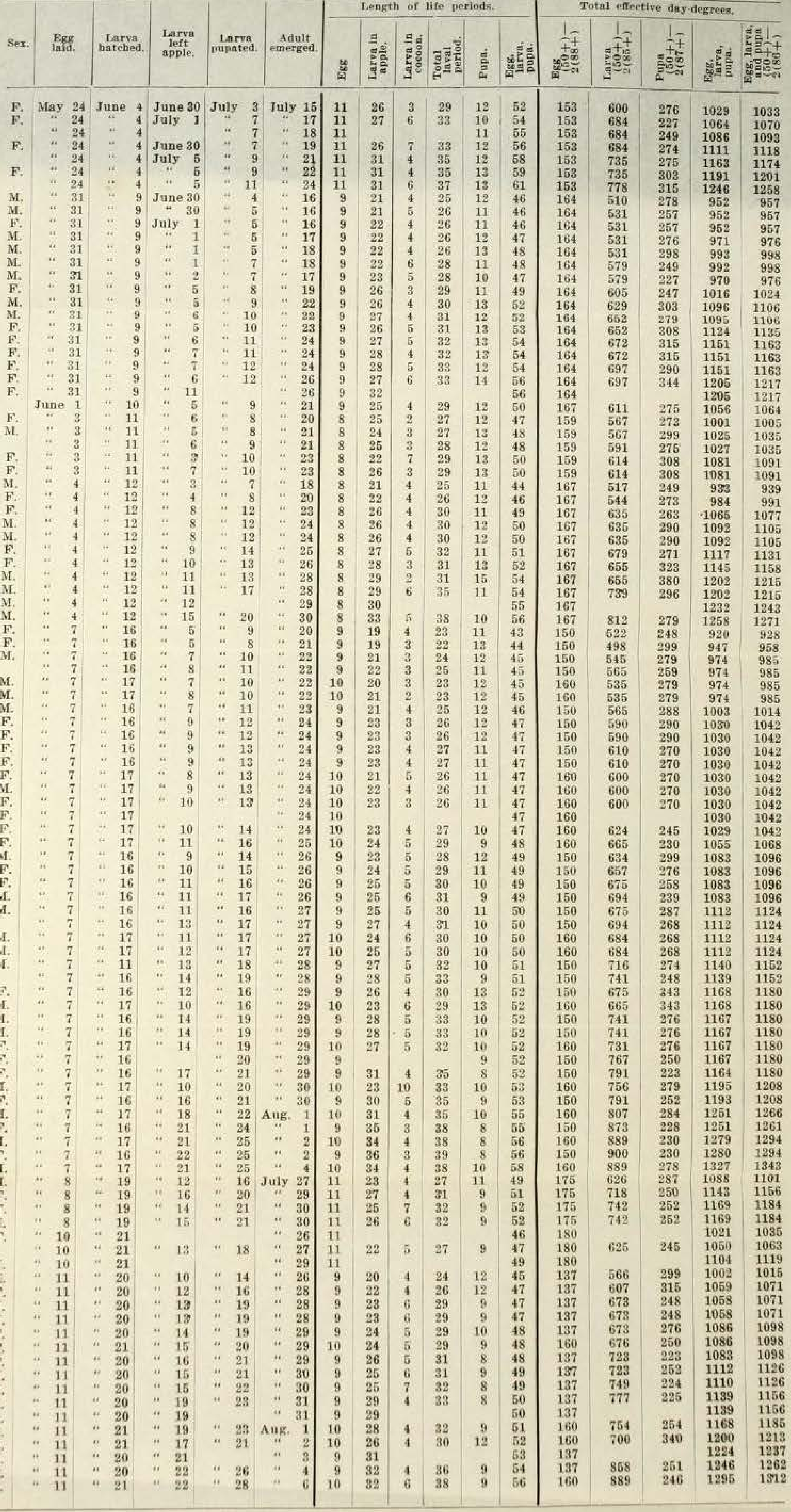

SUMMARY OF TAHER 11

\begin{tabular}{|c|c|c|c|c|c|c|c|c|c|c|c|c|c|c|}
\hline \multirow{3}{*}{ Kex. } & \multicolumn{2}{|c|}{ EkK. } & \multicolumn{2}{|c|}{ Larva is frait. } & \multicolumn{2}{|c|}{ Larva in cocoon. } & \multicolumn{2}{|c|}{ Larva. } & \multicolumn{2}{|c|}{ Fupn. } & \multicolumn{4}{|c|}{ Desg, larvia, and pupa. } \\
\hline & & & & & & & & & & & & & $\begin{array}{c}\text { Total } \\
\text { day. }\end{array}$ & $\begin{array}{l}\text { Tective } \\
\text { sreess. }\end{array}$ \\
\hline & No. & $\begin{array}{l}\text { lis. } \\
\text { days. }\end{array}$ & No. & $\begin{array}{c}\text { in } \\
\text { deys. }\end{array}$ & No. & $\begin{array}{l}I_{0} \\
\text { dinya. }\end{array}$ & No. & $\begin{array}{c}\text { In } \\
\text { anya. }\end{array}$ & No. & $\begin{array}{l}\text { In } \\
\text { diny. }\end{array}$ & No, & in ${ }_{\text {days. }}$ & $\begin{array}{l}\text { Sum for } \\
\text { atiages. }\end{array}$ & $60+5$ \\
\hline
\end{tabular}


TABle 12 - Life-histoby Data on First Generation, Olney, 1915. Labvae reaked in Picked Apples

\begin{tabular}{|c|c|c|c|c|c|c|c|c|c|c|c|c|c|c|c|c|c|c|c|c|}
\hline \multirow[b]{2}{*}{ Sex. } & \multirow{2}{*}{\multicolumn{2}{|c|}{$\begin{array}{l}\text { Egg } \\
\text { laid. }\end{array}$}} & \multirow{2}{*}{\multicolumn{2}{|c|}{$\begin{array}{c}\text { Larva } \\
\text { hatched. }\end{array}$}} & \multirow{2}{*}{\multicolumn{2}{|c|}{$\begin{array}{l}\text { Larva } \\
\text { left } \\
\text { apple. }\end{array}$}} & \multirow{2}{*}{\multicolumn{2}{|c|}{$\begin{array}{c}\text { Larva } \\
\text { pupated. }\end{array}$}} & \multirow[b]{2}{*}{$\begin{array}{c}\text { Adult } \\
\text { emerged. }\end{array}$} & \multicolumn{6}{|c|}{ Length of life periods. } & \multicolumn{5}{|c|}{ Total effective day-degrees. } \\
\hline & & & & & & & & & & $\underset{\infty}{\infty}$ & 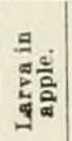 & 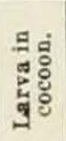 & 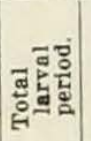 & 혈. & 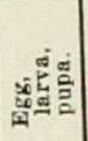 & 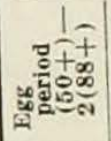 & 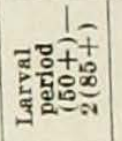 & 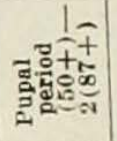 & 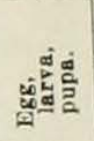 & 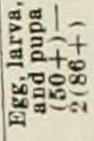 \\
\hline M. & June & 5 & June & 13 & & & July & 8 & July 18 & 8 & & & 25 & 10 & 43 & 157 & 501 & 265 & 923 & 919 \\
\hline M. & " & 5 & & 13 & & & & 8 & “ 18 & 8 & & & 25 & 10 & 43 & 157 & 501 & 265 & 923 & 919 \\
\hline F. & " & 5 & " & 13 & July & 3 & ". & 8 & 18 & 8 & 20 & 5 & 25 & 10 & 43 & 157 & 501 & 265 & 923 & 919 \\
\hline M. & ". & 5 & " & 13 & & 3 & " & 10 & 20 & 8 & 20 & 7 & 27 & 10 & 45 & 157 & 541 & 279 & 977 & 973 \\
\hline M. & “ & 5 & " & 13 & & & “ & 11 & 21 & 8 & & & 28 & 10 & 46 & 157 & 563 & 276 & 996 & 991 \\
\hline M. & .. & 5 & “. & 13 & & & " & 12 & 22 & 8 & & & 29 & 10 & 48 & 157 & 586 & 271 & 1014 & 1010 \\
\hline F. & . & 5 & ." & 13 & " & 8 & " & 12 & 22 & 8 & 25 & 4 & 29 & 10 & 47 & 157 & 586 & 271 & 1014 & 1010 \\
\hline F. & " & 5 & ". & 13 & ". & 8 & ". & 13 & 23 & 8 & 25 & 5 & 30 & 10 & 48 & 157 & 613 & 264 & 1034 & 1030 \\
\hline M. & $"$ & 5 & ". & 13 & " & 11 & " & 14 & 24 & 8 & 28 & 3 & 31 & 10 & 49 & 157 & 642 & 256 & 1055 & 1052 \\
\hline M. & ." & 5 & “ & 13 & “. & 10 & "* & 14 & 26 & 8 & 27 & 4 & 31 & 12 & 51 & 157 & 642 & 304 & 1103 & 1100 \\
\hline F. & ". & 5 & " & 13 & & & ". & 18 & 28 & 8 & & & 35 & 10 & 53 & 157 & 759 & 235 & 1151 & 1153 \\
\hline M. & ". & 7 & ". & 15 & " & 10 & “. & 13 & 23 & 8 & 25 & 3 & 28 & 10 & 46 & 151 & 572 & 264 & 987 & 984 \\
\hline F. & ". & 13 & “ & 22 & " & 13 & “ & 16 & 29 & 9 & 21 & 3 & 24 & 13 & 46 & 195 & 505 & 325 & 1025 & 1026 \\
\hline M. & " & 13 & “ & 21 & & & ." & 18 & 29 & 8 & & & 27 & 11 & 46 & 173 & 586 & 264 & 1023 & 1026 \\
\hline F. & ". & 13 & “ & 20 & & & “. & 18 & 29 & 7 & & & 28 & 11 & 46 & 148 & 610 & 264 & 1022 & 1026 \\
\hline F. & .. & 13 & ". & 21 & “. & 15 & “ & 18 & 29 & 8 & 24 & 3 & 27 & 11 & 46 & 173 & 586 & 264 & 1023 & 1026 \\
\hline F. & .. & 13 & ". & & & & “ & 19 & 30 & 8 & & & 28 & 11 & 47 & 173 & 613 & 266 & 1052 & 1055 \\
\hline M. & " & 14 & “. & 22 & & & “ & 14 & 24 & 8 & & & 22 & 10 & 40 & 173 & 447 & 256 & 876 & 874 \\
\hline F. & ". & 14 & “ & 21 & & & ". & 16 & 27 & 7 & & & 25 & 11 & 43 & 151 & 527 & 269 & 947 & 949 \\
\hline F. & ". & 14 & ". & 22 & & & “ & 17 & 28 & 8 & & & 25 & 11 & 44 & 173 & 547 & 265 & 985 & 975 \\
\hline M. & “ & 14 & " & 22 & ". & 16 & " & 18 & 30 & 8 & 24 & 2 & 26 & 12 & 46 & 173 & 576 & 293 & 1042 & 1033 \\
\hline F. & ". & 14 & “ & 23 & & & $"$ & 18 & 30 & 9 & & & 25 & 12 & 46 & 191 & 558 & 293 & 1042 & 1033 \\
\hline F. & “ & 14 & ". & 22 & “ & 16 & " & 19 & 30 & 8 & 24 & 3 & 27 & 11 & 46 & 173 & 591 & 266 & 1030 & 1033 \\
\hline F. & ." & 15 & " & 23 & " & 13 & $"$ & 16 & 26 & 8 & 20 & 3 & 23 & 10 & 41 & 172 & 488 & 242 & 902 & 903 \\
\hline M. & " & 15 & $"$ & 23 & ". & 15 & “ & 18 & 29 & 8 & 22 & 3 & 25 & 11 & 44 & 172 & 558 & 264 & 984 & 985 \\
\hline F. & . & 16 & 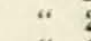 & 24 & “ & 13 & “ & 16 & 26 & 9 & 19 & 3 & 22 & 10 & 40 & 170 & 470 & 242 & 882 & 884 \\
\hline F. & " & 16 & " & 23 & & & " & 18 & 28 & 7 & & & 25 & 10 & 42 & 153 & 558 & 235 & 946 & 937 \\
\hline M. & “ & 16 & " & 23 & & & ". & 18 & " $\quad 29$ & 7 & & & 25 & 11 & 43 & 153 & 558 & 264 & 975 & 966 \\
\hline M. & “ & 16 & “ & 24 & ". & 22 & " & 26 & Aug. 6 & 8 & 28 & 4 & 32 & 11 & 53 & 170 & 710 & 272 & 1152 & 1154 \\
\hline F. & $"$ & 16 & .. & 23 & “. & 16 & “ & 19 & July 30 & 7 & 23 & 3 & 26 & 11 & 44 & 153 & 573 & 266 & 992 & 996 \\
\hline F. & $*$ & 16 & $"$ & 23 & & & " & 30 & Aug. 10 & 7 & & & 37 & 11 & 55 & 153 & 837 & 259 & 1249 & 1248 \\
\hline
\end{tabular}

SUMMABY OF TABLE 12

\begin{tabular}{|c|c|c|c|c|c|c|c|c|c|c|c|c|c|c|}
\hline \multirow{3}{*}{ Sex. } & \multicolumn{2}{|c|}{ Egg. } & \multicolumn{2}{|c|}{ Larva in fruit. } & \multicolumn{2}{|c|}{ Larva in cocoon. } & \multicolumn{2}{|c|}{ Larva. } & \multicolumn{2}{|c|}{ Pupa. } & \multicolumn{4}{|c|}{ Egg, larva, and pupa. } \\
\hline & \multirow{2}{*}{ No. } & \multirow{2}{*}{$\begin{array}{c}\text { Period } \\
\text { in } \\
\text { days. }\end{array}$} & \multirow{2}{*}{ No. } & \multirow{2}{*}{$\begin{array}{c}\text { Period } \\
\text { in } \\
\text { days. }\end{array}$} & \multirow{2}{*}{ No. } & \multirow{2}{*}{$\begin{array}{c}\text { Period } \\
\text { In } \\
\text { days. }\end{array}$} & \multirow{2}{*}{ No. } & \multirow{2}{*}{$\begin{array}{c}\text { Period } \\
\text { In } \\
\text { days. }\end{array}$} & \multirow{2}{*}{ No. } & \multirow{2}{*}{$\begin{array}{c}\text { Period } \\
\text { in } \\
\text { days. }\end{array}$} & \multirow{2}{*}{ No. } & \multirow{2}{*}{$\begin{array}{c}\text { Period } \\
\text { In } \\
\text { days. }\end{array}$} & \multicolumn{2}{|c|}{$\begin{array}{l}\text { Total effective } \\
\text { day-degrees. }\end{array}$} \\
\hline & & & & & & & & & & & & & $\begin{array}{l}\text { Sum for } \\
\text { stages. }\end{array}$ & $\begin{array}{l}(50+)- \\
2(86+) .\end{array}$ \\
\hline M. & 14 & 8.0 & 7 & 24.9 & 7 & 3.7 & 14 & 27.2 & 14 & 10.6 & 14 & 45.9 & 1002 & 999 \\
\hline F. & 17 & 7.8 & 9 & 22.4 & 9 & 3.6 & 17 & 27.1 & 17 & 10.8 & 17 & 45.7 & 1013 & 1012 \\
\hline M. F. & 31 & 7.9 & 16 & 23.5 & 16 & 3.6 & 31 & 27.1 & 31 & 10.7 & 31 & 45.8 & 1008 & 1006 \\
\hline
\end{tabular}




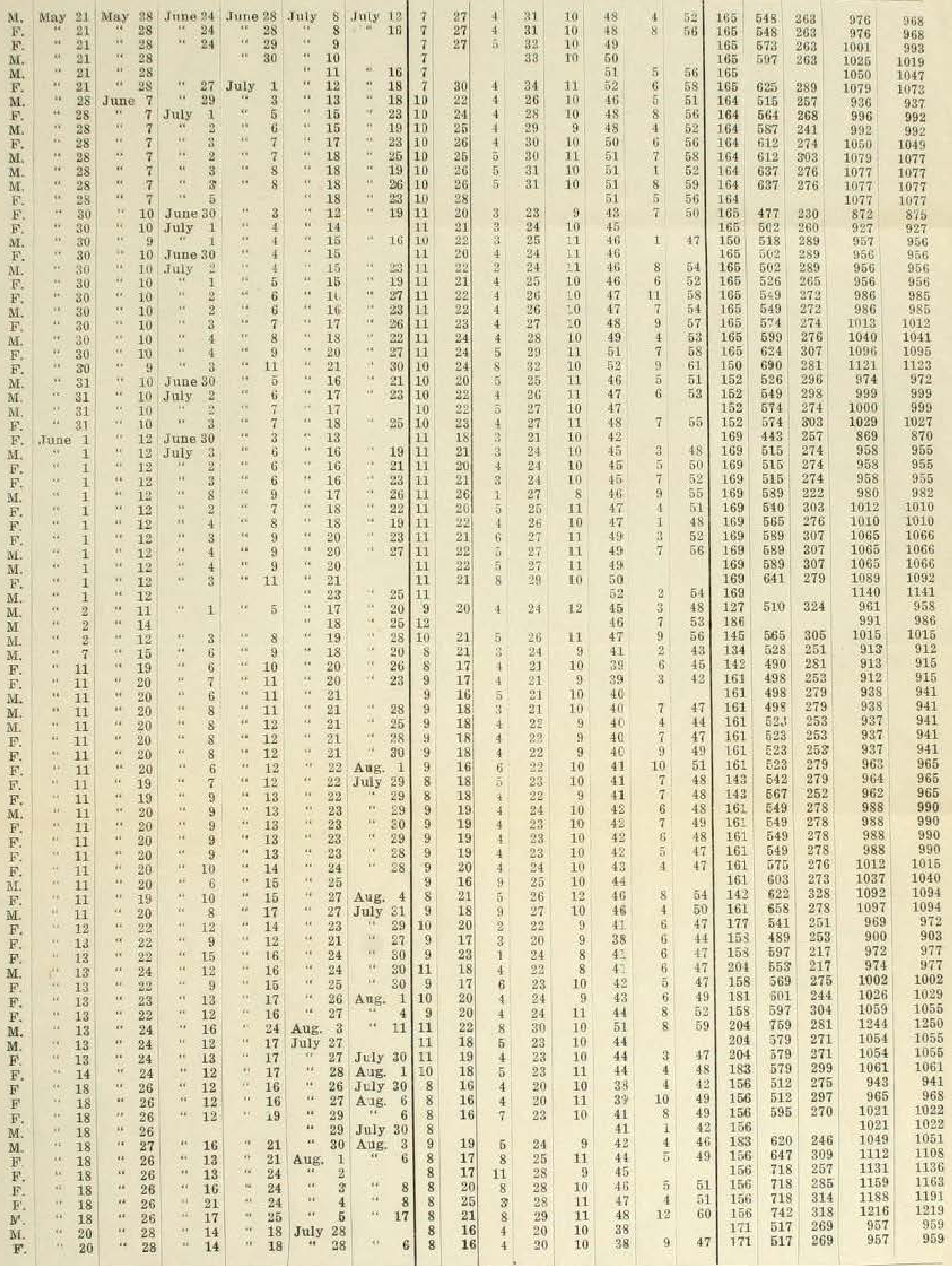

SUMMABY OF TABLE 12

\begin{tabular}{l} 
Egk. Larva in fruit, Larva in cocoon: Larva. \\
\hline
\end{tabular} 
TABLE 13 - Oviposition and Life Proiod of Auults of Flast Genebation, Olney, 1915.

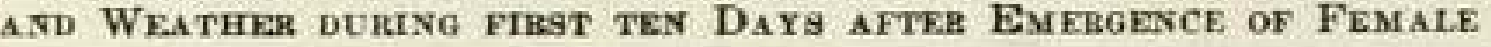

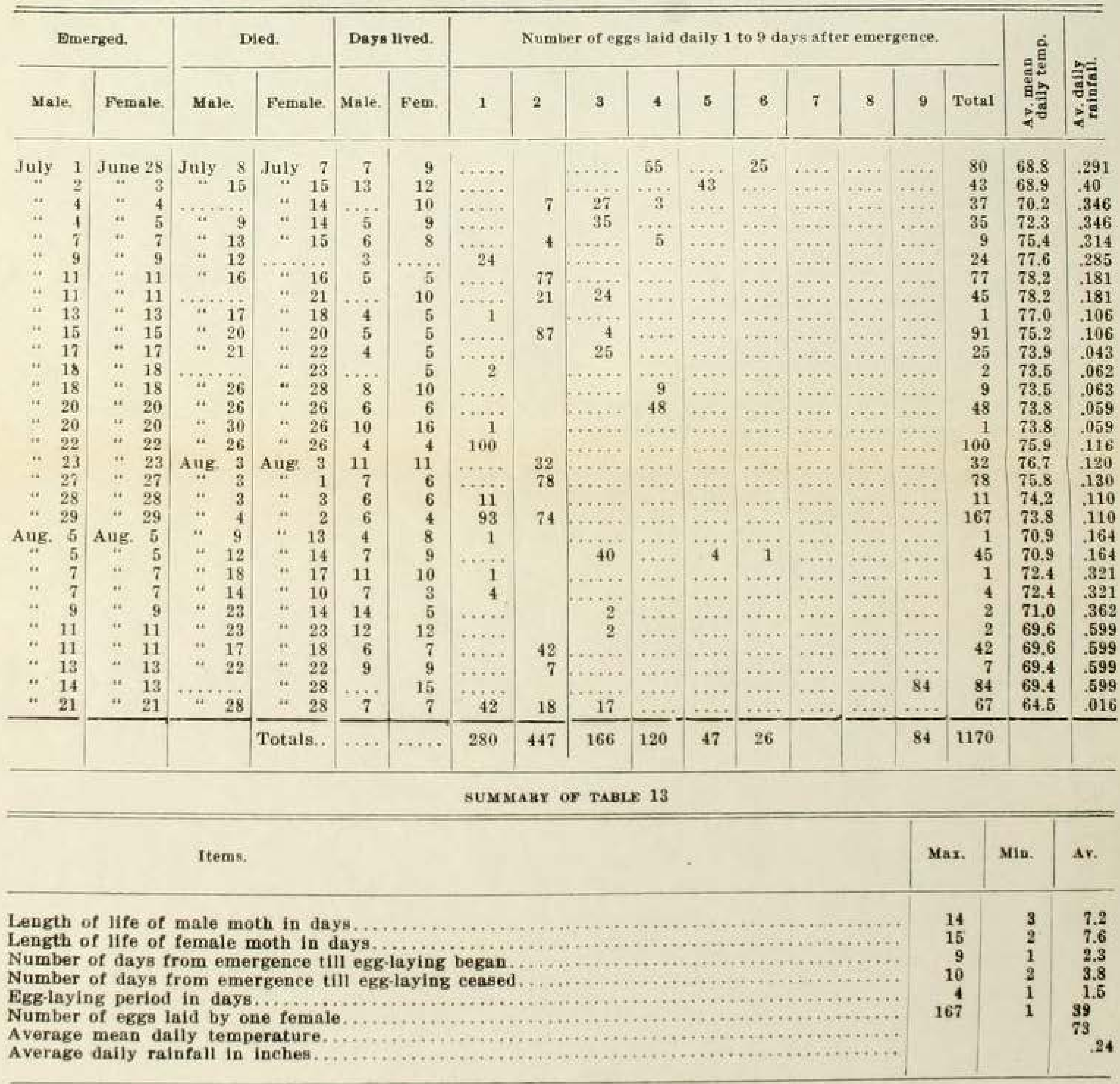




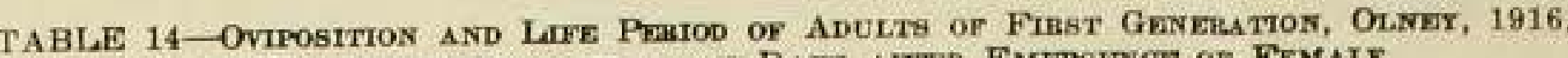
AND WEATHER DURANG FTRST TEN DAYs AYTER EMBDOENCE OF FEMALE

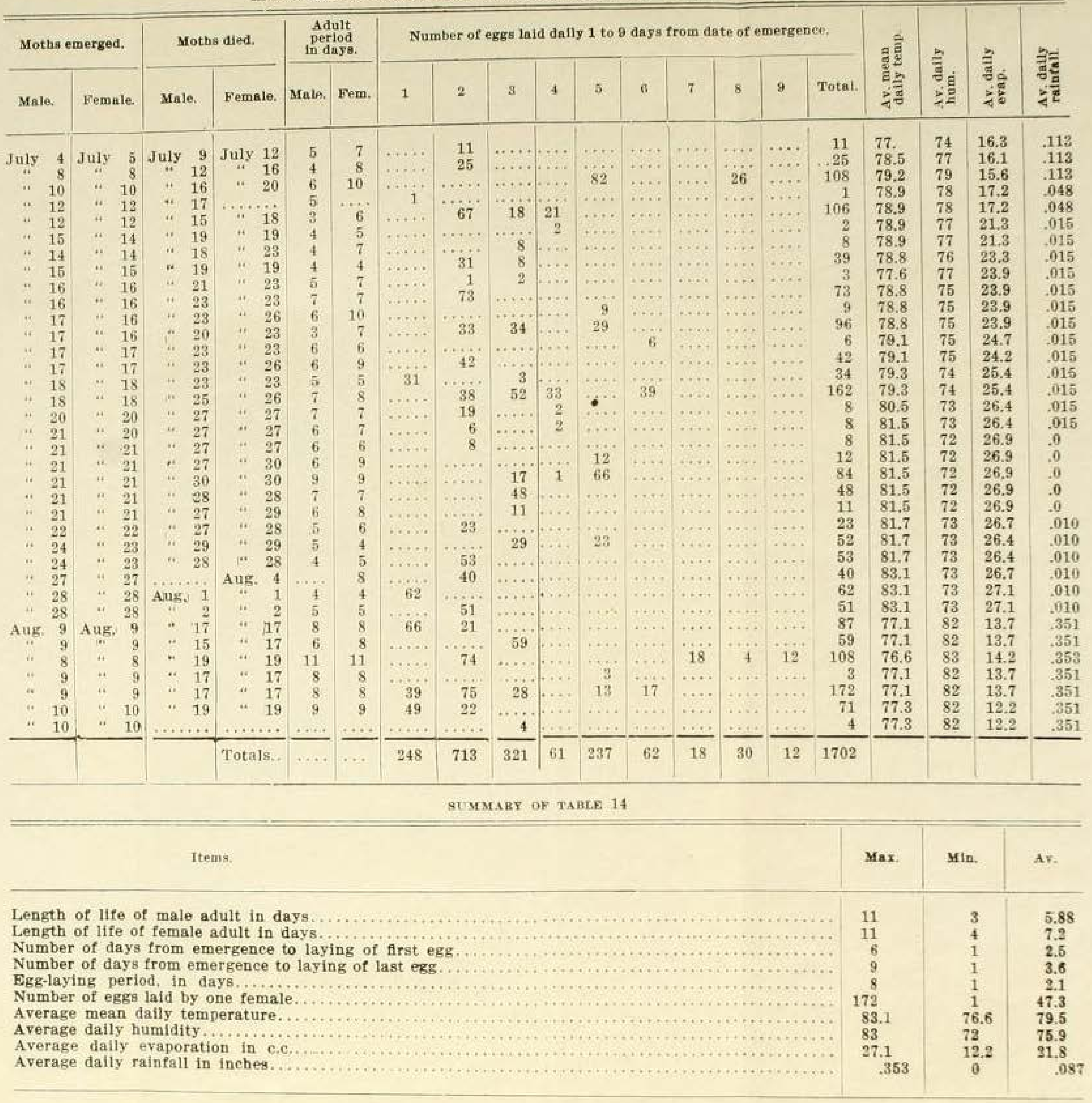


TABLe 15-Life-history Data on Portion of Second Genfration whose Larvae transformed, Olney, 1915. latrvae raeared in apples on Tree

\begin{tabular}{|c|c|c|c|c|c|c|c|c|c|c|c|c|c|c|c|c|c|c|c|c|}
\hline \multirow[b]{2}{*}{ Sex. } & \multirow{2}{*}{\multicolumn{2}{|c|}{$\begin{array}{l}\text { Egg } \\
\text { laid. }\end{array}$}} & \multirow{2}{*}{\multicolumn{2}{|c|}{$\begin{array}{c}\text { Larva } \\
\text { hatched. }\end{array}$}} & \multirow{2}{*}{\multicolumn{2}{|c|}{$\begin{array}{l}\text { Larva } \\
\text { left } \\
\text { apple. }\end{array}$}} & \multirow{2}{*}{\multicolumn{2}{|c|}{$\begin{array}{c}\text { Larva } \\
\text { pupated }\end{array}$}} & \multirow[b]{2}{*}{$\begin{array}{c}\text { Adult } \\
\text { emerged. }\end{array}$} & \multicolumn{6}{|c|}{ Length of life periods. } & \multicolumn{5}{|c|}{ Total effective day-degrees. } \\
\hline & & & & & & & & & & 离 & 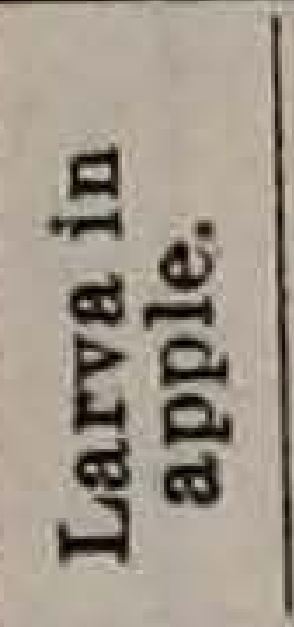 & 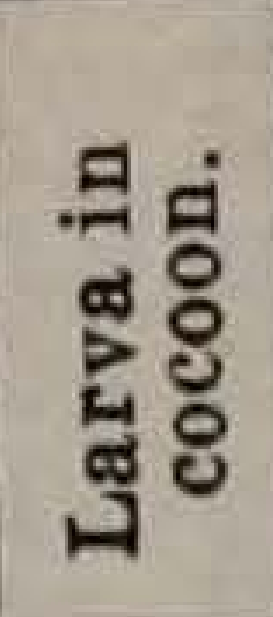 & 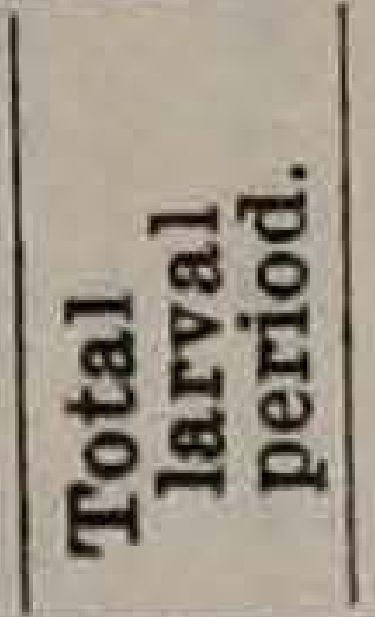 & ڤึ่ & 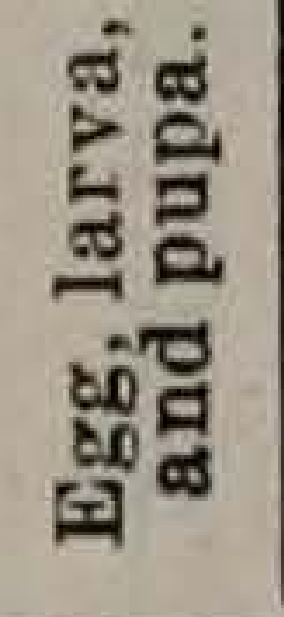 & 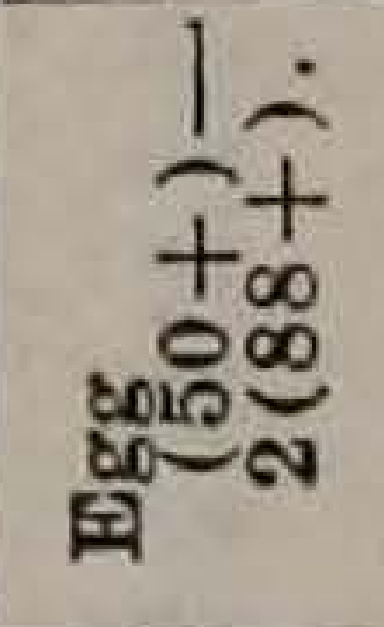 & 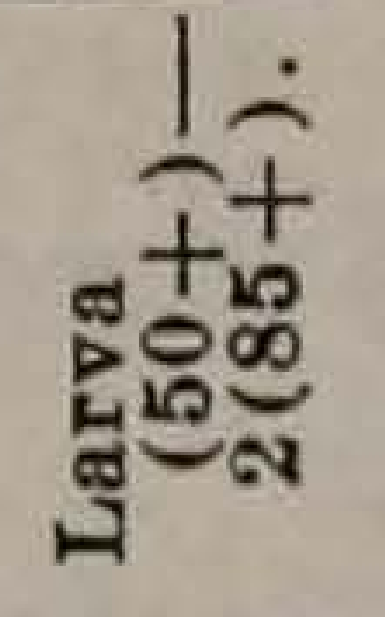 & 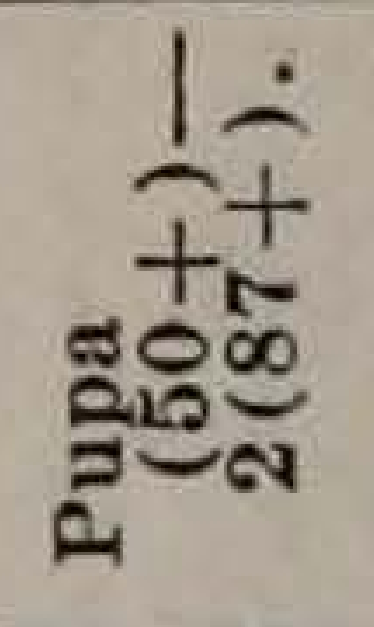 & 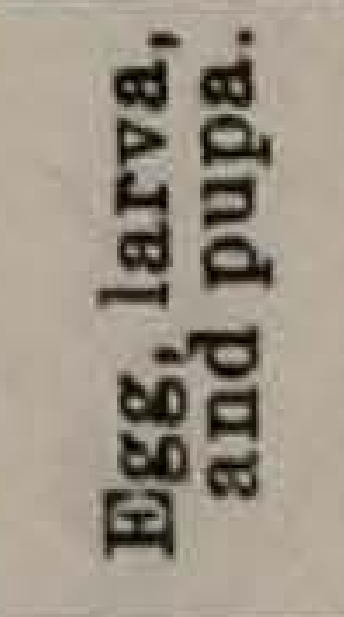 & 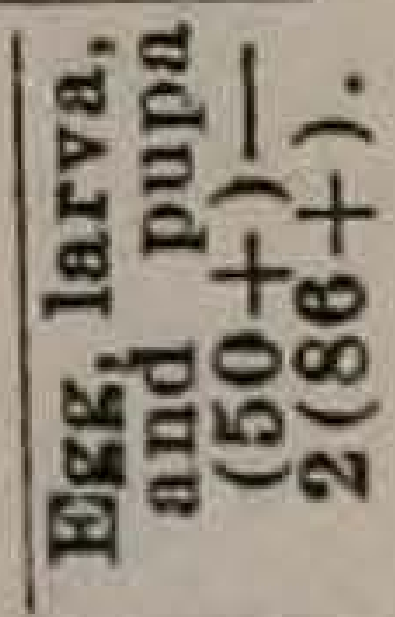 \\
\hline & July & 2 & July & 11 & July & 31 & Aug. & 5 & & 9 & 20 & 5 & 25 & & & 161 & 628 & & & \\
\hline F. & " & 7 & & 14 & Aug. & 3 & " & 8 & Aug. 19 & 7 & 20 & 5 & 25 & 11 & 43 & 162 & 616 & 231 & 1009 & 1009 \\
\hline M. & “ & 7 & & 14 & " & 7 & " & 10 & " 24 & 7 & 24 & 3 & 27 & 14 & 48 & 162 & 664 & 273 & 1099 & 1099 \\
\hline F. & ". & 7 & " & 13 & “ & 9 & " & 13 & 27 & 6 & 27 & 4 & 31 & 14 & 51 & 132 & 750 & 257 & 1139 & 1140 \\
\hline M. & “ & 7 & & 14 & & & & & 21 & 7 & & & & & 45 & 162 & & & 1041 & 1041 \\
\hline F. & “ & 7 & " & 14 & " & 5 & " & 10 & 23 & 7 & 22 & 5 & 27 & 13 & 47 & 162 & 664 & 250 & 1076 & 1076 \\
\hline M. & " & 7 & " & 14 & " & 4 & " & 10 & 24 & 7 & 21 & 6 & 27 & 14 & 48 & 162 & 664 & 273 & 1099 & 1099 \\
\hline F. & “ & 7 & “ & 14 & “ & 9 & “ & 13 & “ 27 & 7 & 26 & 4 & 30 & 14 & 51 & 162 & 721 & 257 & 1140 & 1140 \\
\hline M. & " & 7 & " & 14 & “ & 14 & “ & 17 & Sept. 4 & 7 & 31 & 3 & 34 & 18 & 59 & 162 & 815 & 277 & 1254 & 1253 \\
\hline F. & " & 8 & “ & 14 & " & 2 & “ & 3 & Aug. 16 & 6 & 19 & 1 & 20 & 13 & 39 & 144 & 520 & 270 & 934 & 934 \\
\hline$\vec{M}$. & “ & 8 & " & 14 & & & & & " 22 & 6 & & & & & 45 & 144 & & & 1039 & 1039 \\
\hline F. & " & 8 & “ & 14 & " & 9 & “ & 16 & “ 27 & 6 & 26 & 7 & 33 & 11 & 50 & 144 & 791 & 188 & 1123 & 1122 \\
\hline F. & " & 8 & " & 14 & " & 13 & “ & 17 & Sept. 2 & 6 & 30 & 4 & 34 & 16 & 56 & 144 & 815 & 238 & 1197 & 1197 \\
\hline F. & “ & 10 & “ & 17 & “ & 14 & “ & 17 & Sept. 3 & 7 & 28 & 3 & 31 & 17 & 55 & 196 & 727 & 255 & 1178 & 1175 \\
\hline
\end{tabular}

SUMMARY OF TARLE 15

\begin{tabular}{|c|c|c|c|c|c|c|c|c|c|c|c|c|c|c|}
\hline \multirow{3}{*}{ Sex. } & \multicolumn{2}{|c|}{ Egg. } & \multicolumn{2}{|c|}{ Larva in fruit. } & \multicolumn{2}{|c|}{ Larva in cocoon. } & \multicolumn{2}{|c|}{ Larva. } & \multicolumn{2}{|c|}{ Pupa. } & \multicolumn{4}{|c|}{ Egg, larva, and pupa. } \\
\hline & \multirow[b]{2}{*}{ No. } & \multirow{2}{*}{$\begin{array}{l}\text { Period } \\
\text { in } \\
\text { days. }\end{array}$} & \multirow[b]{2}{*}{ No. } & \multirow{2}{*}{$\begin{array}{c}\text { Period } \\
\text { in } \\
\text { days. }\end{array}$} & \multirow[b]{2}{*}{ No. } & \multirow{2}{*}{$\begin{array}{c}\text { Period } \\
\text { in } \\
\text { days. }\end{array}$} & \multirow[b]{2}{*}{ No. } & \multirow{2}{*}{$\begin{array}{c}\text { Period } \\
\text { in } \\
\text { days. }\end{array}$} & \multirow[b]{2}{*}{ No. } & \multirow{2}{*}{$\begin{array}{c}\text { Period } \\
\text { in } \\
\text { days. }\end{array}$} & \multirow[b]{2}{*}{ No. } & \multirow{2}{*}{$\begin{array}{c}\text { Period } \\
\text { in } \\
\text { days. }\end{array}$} & \multicolumn{2}{|c|}{$\begin{array}{l}\text { Total effective } \\
\text { day-degrees. }\end{array}$} \\
\hline & & & & & & & & & & & & & $\begin{array}{l}\text { Sum for } \\
\text { stages. }\end{array}$ & $\begin{array}{l}(50+)- \\
2(86+)\end{array}$ \\
\hline M. & 5 & 6.8 & 3 & 25.3 & 3 & 4.0 & 3 & 29.3 & 3 & 15.3 & 5 & 49 & 1106 & 1106 \\
\hline F. & 8 & 6.5 & 8 & 24.8 & 8 & 4.1 & 8 & 29.0 & 8 & 13.6 & 8 & 49 & 1100 & 1099 \\
\hline Und. & 1 & 9.0 & 1 & 20.0 & 1 & 5.0 & 1 & 25.0 & 0 & & 0 & & & \\
\hline M.\& F. & 14 & 6.8 & 12 & 24.5 & 12 & 4.2 & 12 & 28.7 & 11 & 14.1 & 13 & 49 & 1102 & 1102 \\
\hline
\end{tabular}




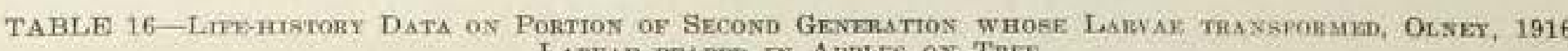

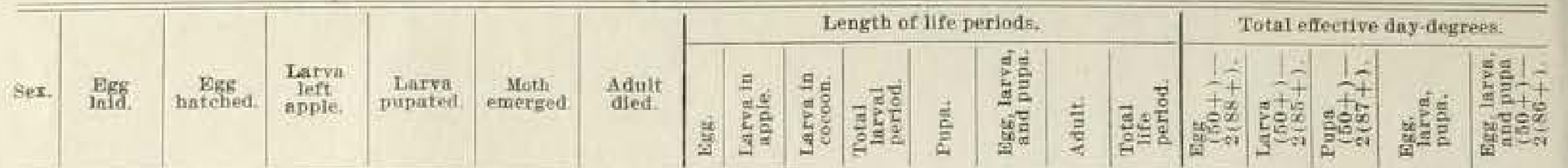

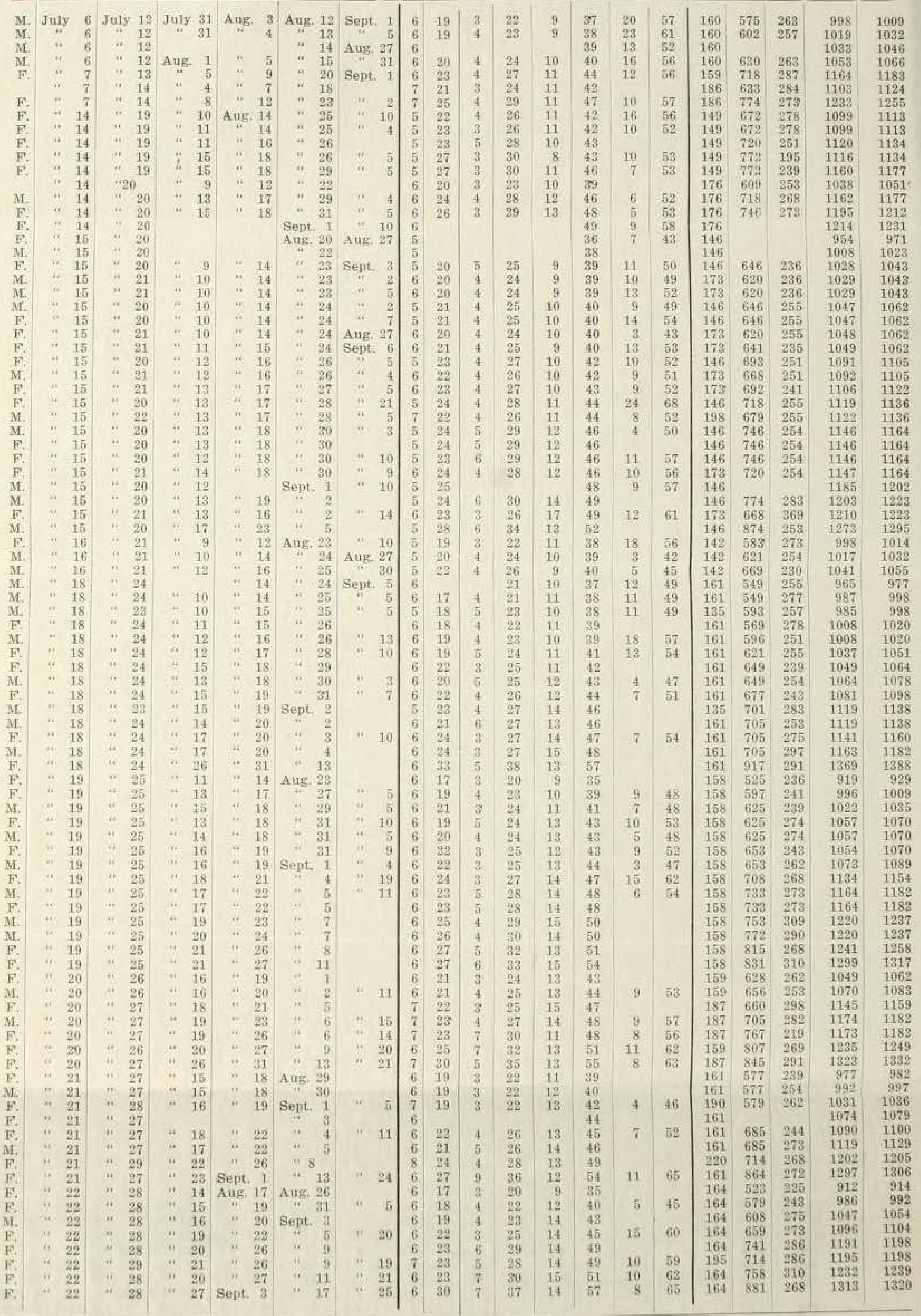

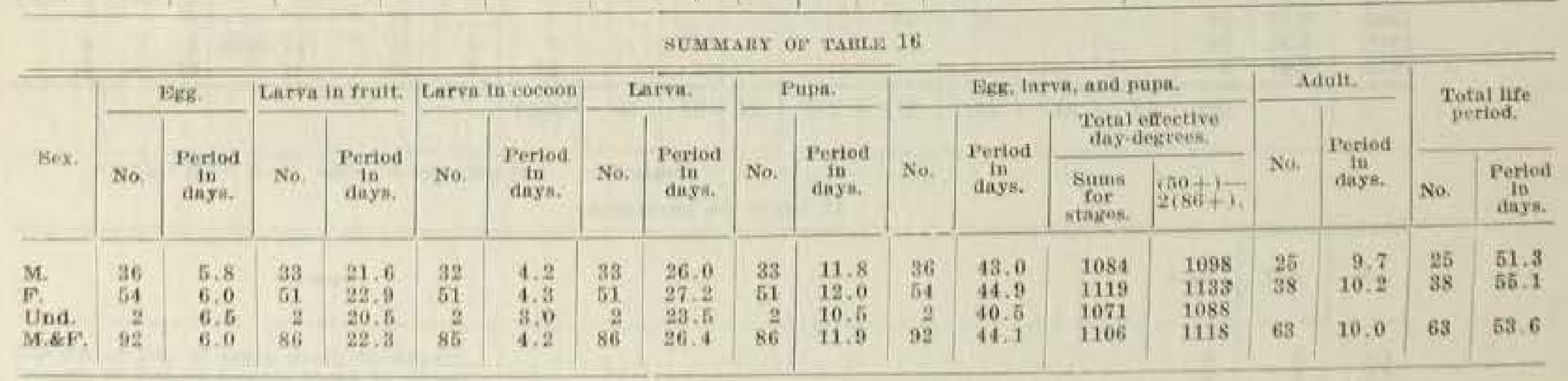


TABLe 17-Oviposition and Lafe Period of Adults of Second Generation, Olney, 1915,

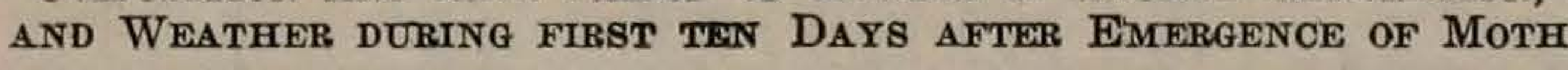

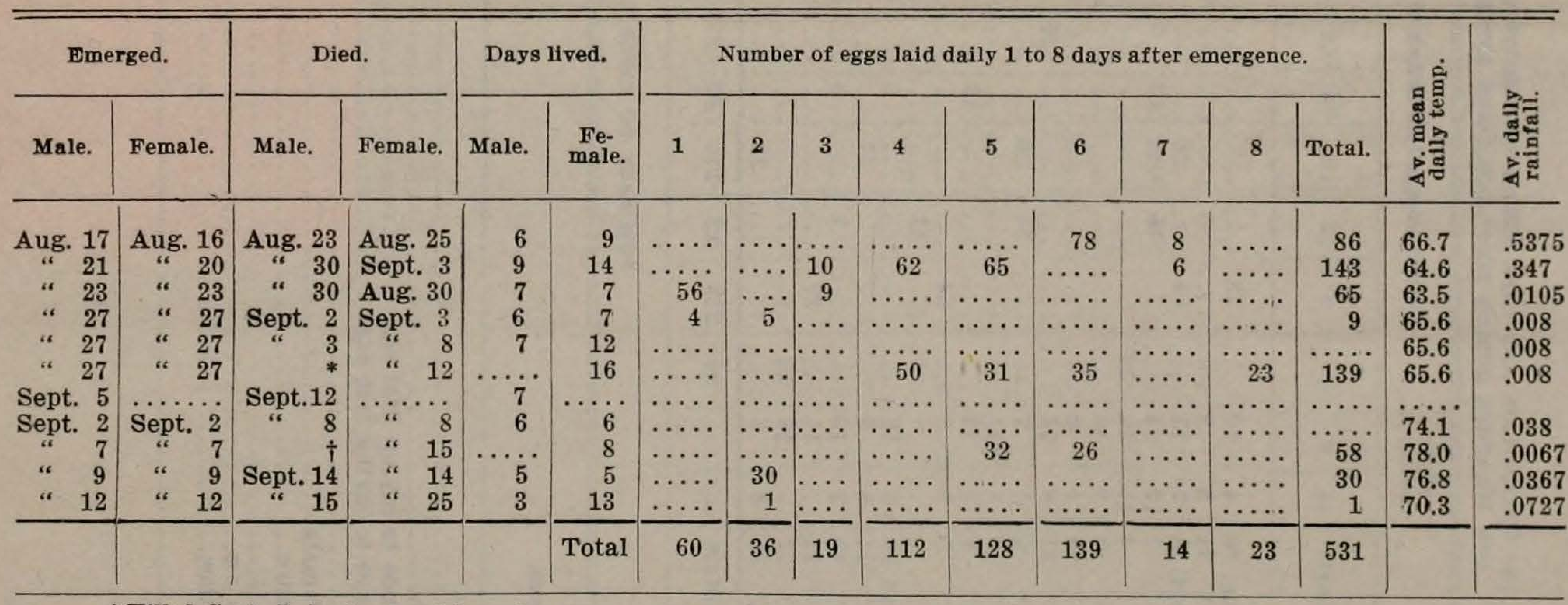

* Killed Sept. 5, by larva of lacewing fly.

$\dagger$ Escaped.

SUMMARY OF TABLE 17

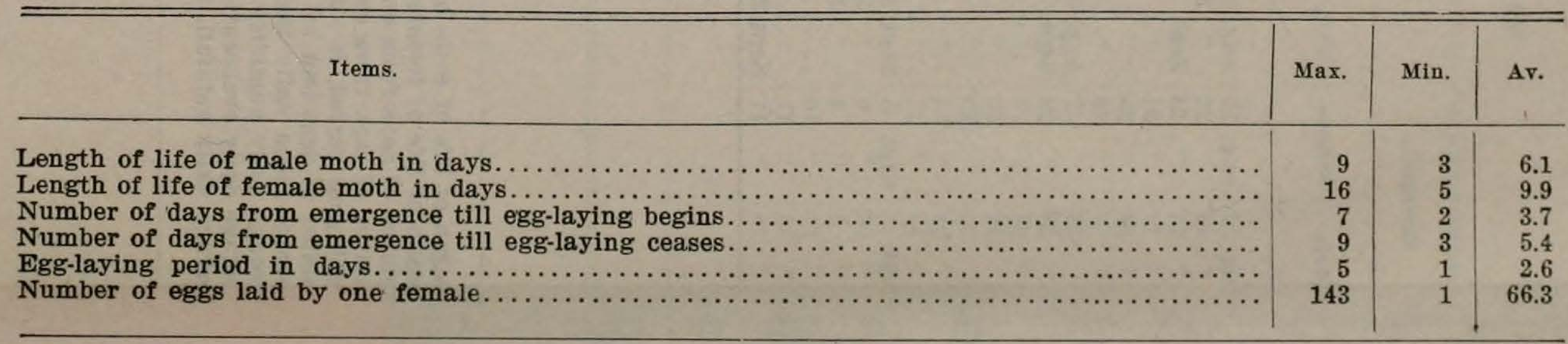


TABLE 18-Oviposition and Life Period of Aduits of Second Generation, Olney, 1916. AND WEATHEB DURING FLBST TEN DAYS AFTEB EMIFBOENCE OE FEMALP

\begin{tabular}{|c|c|c|c|c|c|c|c|c|c|c|c|c|c|c|c|c|c|c|c|c|c|c|}
\hline \multicolumn{2}{|c|}{ Emerged. } & \multicolumn{2}{|c|}{ Died } & \multicolumn{2}{|c|}{$\begin{array}{l}\text { Adult } \\
\text { period. }\end{array}$} & \multicolumn{13}{|c|}{ Number of eggs laid dally 1 to 12 days from date of emergence. } & \multirow{2}{*}{ 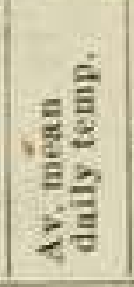 } & \multirow{2}{*}{ 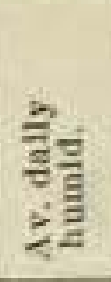 } & \multirow{2}{*}{ 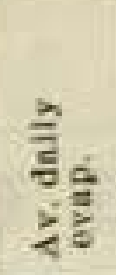 } & \multirow{2}{*}{ 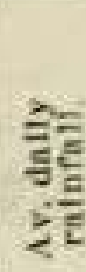 } \\
\hline Male. & Female & Male. & Female. & M. & v. & 1. & 2 & 3 & 4 & 5 & 6 & 7 & 8 & 9 & 10 & 11 & 12 & Total & & & & \\
\hline 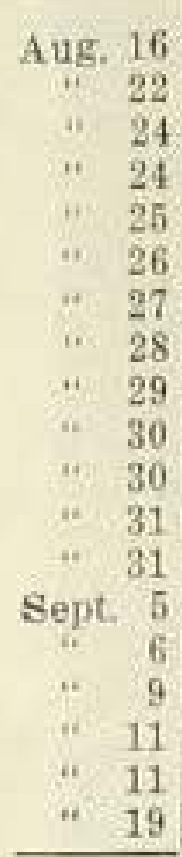 & 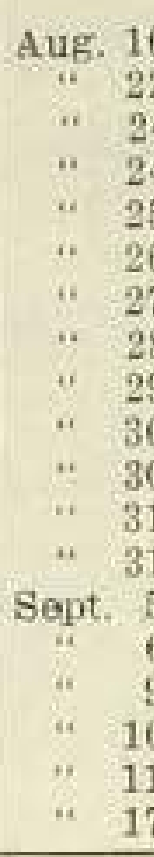 & $\begin{array}{cc}\text { Aug. } & 20 \\
\text { ". } & 31 \\
\text { Sept. } & 2 \\
\text {.. } & 5 \\
. . & 5 \\
. . & 4 \\
\text { Aug. } & 31 \\
\text { Sept. } & 5 \\
\text {. } & 5 \\
\text { ". } & 3 \\
\text {." } & 5 \\
\end{array}$ & $\begin{array}{cr}\text { Aug. } & 26 \\
\text {.. } & 31 \\
\text { Sept. } & 6 \\
. . & 7 \\
. . & 4 \\
. . & 5 \\
. . & 5 \\
. . & 21 \\
. . & 5 \\
. . & 10 \\
. . & 9 \\
. . & 5\end{array}$ & $\begin{array}{r}4 \\
9 \\
9 \\
12 \\
11 \\
9 \\
4 \\
8 \\
7 \\
4 \\
4 \\
5 \\
6 \\
6 \\
9 \\
3 \\
\ldots \\
\ldots \\
5\end{array}$ & $\begin{array}{r}10 \\
9 \\
13 \\
14 \\
10 \\
10 \\
9 \\
24 \\
7 \\
11 \\
10 \\
5 \\
15 \\
8 \\
10 \\
11 \\
12 \\
8 \\
\end{array}$ & $\begin{array}{l}\ldots \\
\ldots \\
32 \\
\ldots \\
\ldots \ldots \\
\ldots \ldots \\
\ldots \ldots \\
\ldots \ldots \\
\ldots \ldots \\
\ldots \ldots \\
53 \\
\ldots \ldots \\
\end{array}$ & $\begin{array}{c}73 \\
64 \\
\ldots \ldots \\
\ldots \ldots \\
\ldots \ldots \\
\ldots \ldots \\
\ldots \ldots \\
\ldots 4 \\
\ldots \ldots \\
\ldots \ldots \\
\ldots \\
\ldots \ldots \\
\ldots \ldots \\
\end{array}$ & $\begin{array}{c}15 \\
\ldots \\
\ldots \\
\ldots \\
65 \\
\ldots \\
\ldots \\
27 \\
\ldots \\
1 \\
\ldots \\
\ldots \\
\ldots \\
\ldots\end{array}$ & $\begin{array}{c}28 \\
46 \\
\ldots \\
\cdots \\
\cdots \\
20 \\
\cdots \\
\cdots \\
\cdots \\
57 \\
\cdots \\
\cdots \\
19 \\
19 \\
\end{array}$ & $\begin{array}{r}19 \\
\cdots \\
\vdots \\
5 \\
6 \\
\cdots \\
\cdots \\
\cdots\end{array}$ & $\begin{array}{c}\ldots \\
\ldots \\
\ldots \\
\ldots \\
\ldots \\
24 \\
\ldots \\
\ldots \\
\ldots \\
\ldots \\
\ldots \\
\ldots \\
\ldots \\
\ldots\end{array}$ & 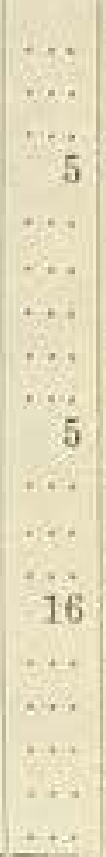 & $\begin{array}{r}2 \\
\ldots \\
\ldots \\
6 \\
20 \\
\ldots \\
10 \\
\ldots \\
\ldots \\
1 \\
1 \\
41 \\
\ldots \\
\ldots \\
\end{array}$ & $\begin{array}{c}\ldots \\
\ldots \\
\ldots \\
\cdots \\
\ldots \\
\ldots \\
\cdots \\
\cdots \\
\cdots \\
1 \\
\ldots \\
\cdots \\
\cdots \\
\cdots \\
\end{array}$ & $\begin{array}{c}\ldots \\
17 \\
\cdots \\
\cdots \\
\cdots \\
\cdots \\
\cdots \\
\cdots \\
\cdots \\
\cdots \\
\cdots \\
\cdots \\
\end{array}$ & $\begin{array}{c}\ldots \\
10 \\
\ldots \\
\ldots \\
\ldots \\
\ldots \\
\ldots \\
\ldots \\
\cdots \\
\ldots \\
\cdots \\
\cdots \\
\end{array}$ & $\begin{array}{c}\ldots . \\
\ldots . \\
10 \\
\ldots \ldots \\
\ldots \ldots \\
\ldots \ldots \\
\ldots \ldots \\
\ldots \ldots \\
\ldots \ldots \\
\ldots \ldots \\
\ldots \ldots \\
\ldots \ldots \\
\ldots \ldots \\
\end{array}$ & $\begin{array}{r}73 \\
2 \\
92 \\
120 \\
32 \\
15 \\
20 \\
24 \\
75 \\
15 \\
19 \\
54 \\
27 \\
23 \\
57 \\
48 \\
37 \\
53 \\
19 \\
\end{array}$ & $\begin{array}{l}76.5 \\
68.0 \\
68.3 \\
68.3 \\
68.3 \\
69.1 \\
70.2 \\
71.6 \\
72.5 \\
72.7 \\
72.7 \\
72.5 \\
72.5 \\
70.2 \\
67.4 \\
62.2 \\
61.0 \\
60.4 \\
61.5\end{array}$ & $\begin{array}{l}80 \\
79 \\
81 \\
81 \\
81 \\
81 \\
80 \\
81 \\
82 \\
83 \\
83 \\
83 \\
83 \\
79 \\
78 \\
74 \\
71 \\
71 \\
70\end{array}$ & $\begin{array}{l}14.8 \\
13.2 \\
11.1 \\
11.1 \\
10.7 \\
11.8 \\
13.6 \\
13.1 \\
12.3 \\
12.2 \\
12.2 \\
11.8 \\
11.8 \\
13.6 \\
13.5 \\
15.2 \\
16.0 \\
17.5 \\
20.1\end{array}$ & $\begin{array}{l}.245 \\
.182 \\
.195 \\
.195 \\
.204 \\
.151 \\
.241 \\
.283 \\
.283 \\
.283 \\
.283 \\
.142 \\
.142 \\
.000 \\
.000 \\
.000 \\
.000\end{array}$ \\
\hline & & & Totals & & & 85 & 228 & 98 & 176 & 42 & 29 & 26 & 80 & 10 & 17 & 10 & 10 & 805 & & & & \\
\hline
\end{tabular}

BUMSARY OF TABLE 18

\begin{tabular}{|c|c|c|c|}
\hline Items. & $\operatorname{Max}$. & Min: & Av. \\
\hline 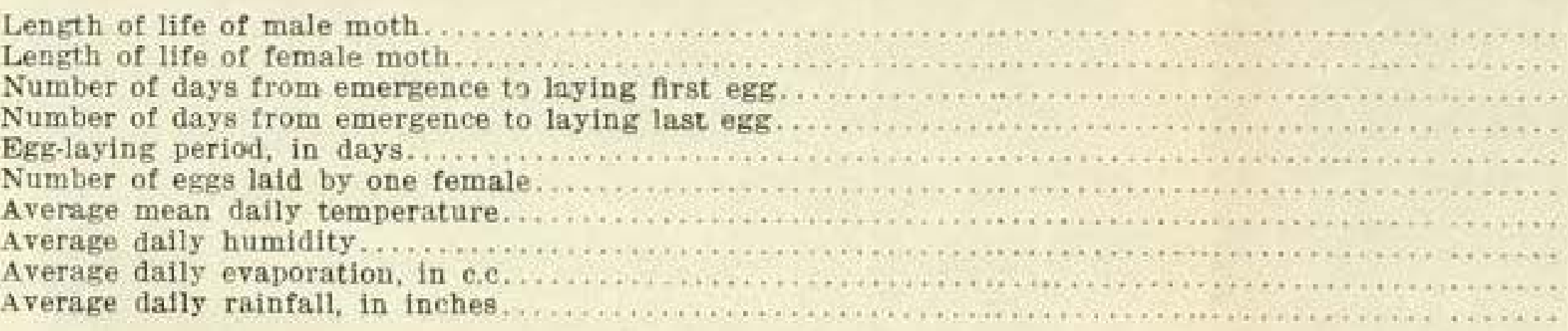 & $\begin{array}{l}12 \\
24 \\
8 \\
12 \\
10 \\
120 \\
72.7 \\
83 \\
20.1 \\
.283\end{array}$ & $\begin{array}{l}3 \\
3 \\
1 \\
1 \\
1 \\
2 \\
60.4 \\
70 \\
10.7 \\
0\end{array}$ & $\begin{array}{r}6.13 \\
11.00 \\
4.1 \\
5.2 \\
2.2 \\
42.3 \\
68.7 \\
79.0 \\
12.9 \\
17.4\end{array}$ \\
\hline
\end{tabular}




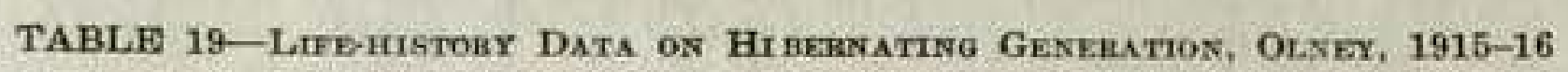

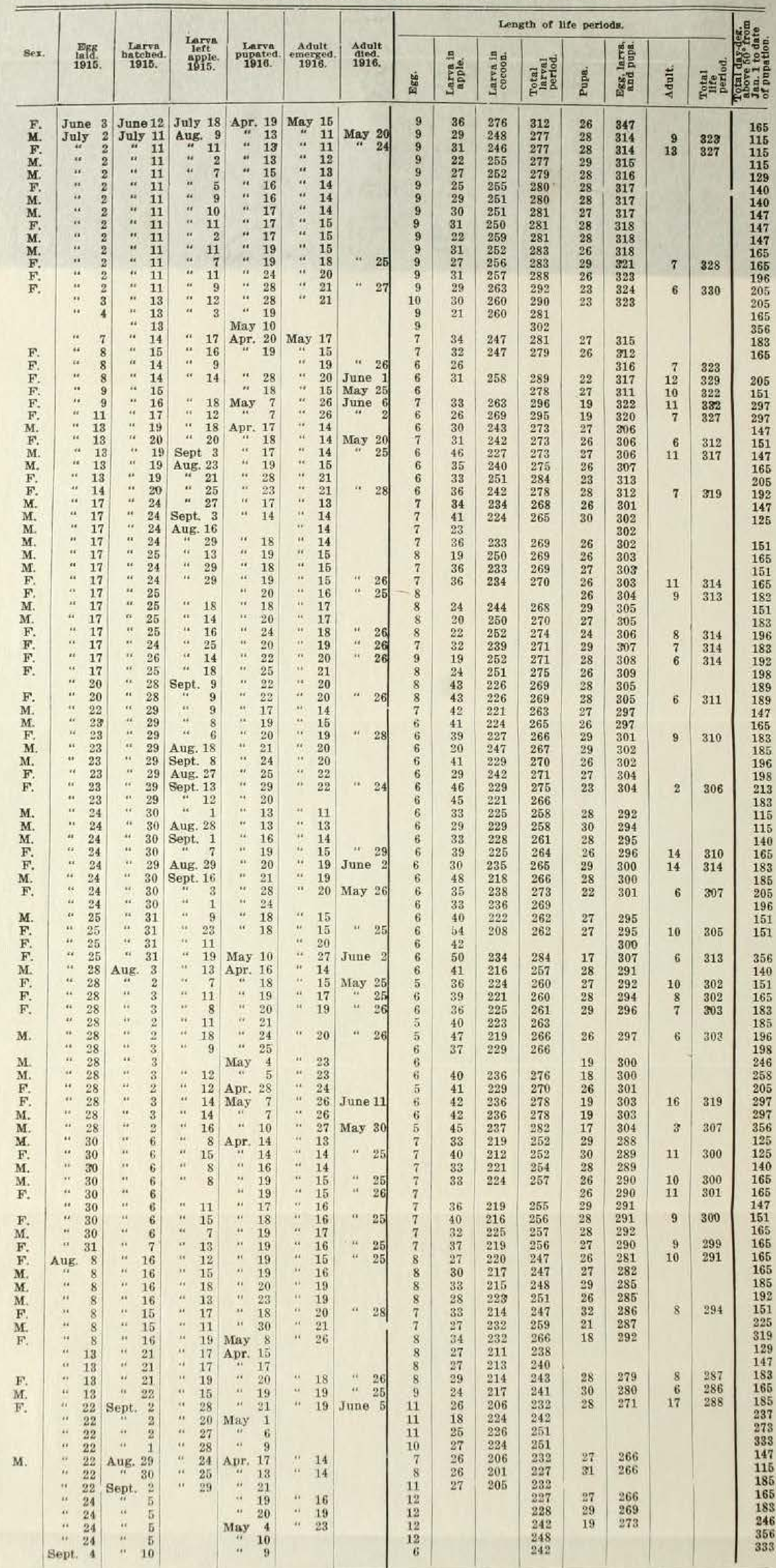

THIX

\begin{tabular}{|c|c|c|c|c|c|c|c|c|c|c|c|c|c|c|c|c|}
\hline \multirow[b]{2}{*}{ Bex. } & \multicolumn{2}{|c|}{ Fuk. } & \multicolumn{2}{|c|}{$\begin{array}{l}\text { Larra in } \\
\text { fruit: }\end{array}$} & \multicolumn{2}{|c|}{$\begin{array}{l}\text { Larva in } \\
\text { cocoon. }\end{array}$} & \multicolumn{2}{|c|}{ Larva. } & \multicolumn{2}{|c|}{ Pupa. } & \multicolumn{2}{|c|}{$\begin{array}{l}\text { Hggg, larva, } \\
\text { and pupa. }\end{array}$} & \multicolumn{2}{|c|}{ Adalt. } & \multicolumn{2}{|c|}{$\begin{array}{l}\text { Total life } \\
\text { period. }\end{array}$} \\
\hline & No. & $\left|\begin{array}{c}\text { Perioa } \\
\text { naty } \\
\text { nayn. }\end{array}\right|$ & No. & $\begin{array}{c}\text { Period } \\
\text { in } \\
\text { dayn }\end{array}$ & No. & $\begin{array}{c}\text { Period } \\
\text { dayn } \\
\text { dayn }\end{array}$ & No. & $\begin{array}{l}\text { Pertod } \\
\text { in } \\
\text { anys. }\end{array}$ & No. & $\begin{array}{l}\text { Period } \\
\text { In } \\
\text { anyn. }\end{array}$ & No. & $\begin{array}{l}\text { Ported } \\
\text { ID. } \\
\text { dayn. }\end{array}$ & $\mathrm{Na}$ & $\begin{array}{c}\text { Period } \\
\text { in: } \\
\text { ings. }\end{array}$ & No. & $\begin{array}{l}\text { Pertod } \\
\text { fit. } \\
\text { days. }\end{array}$ \\
\hline M. & $\begin{array}{l}48 \\
46\end{array}$ & 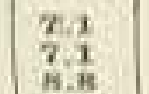 & $\begin{array}{l}43 \\
43 \\
46\end{array}$ & $\begin{array}{l}\text { 32.7 } \\
\text { 3ain } \\
\text { and. }\end{array}$ & i1 & $\begin{array}{l}232.6 \\
2377\end{array}$ & 11 & $\begin{array}{l}2065.5 \\
271.6\end{array}$ & 48 & $\begin{array}{l}\begin{array}{r}36.5 \\
25.8\end{array} \\
25.8\end{array}$ & 46 & $\begin{array}{r}299.3 \\
8004.3\end{array}$ & $35^{6}$ & $9 . \overline{1}$ & 86 & $\begin{array}{l}306.0 \\
310.9\end{array}$ \\
\hline
\end{tabular}




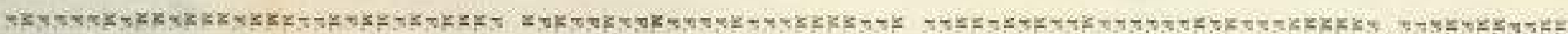

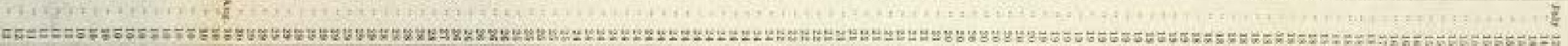
:

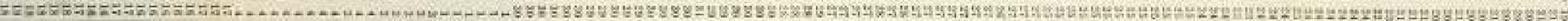

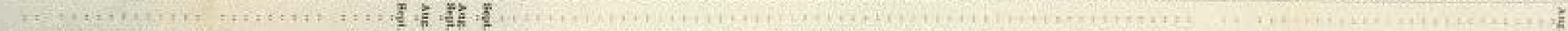

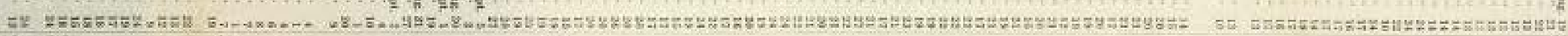
存

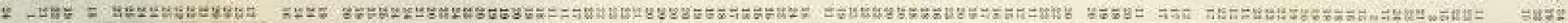

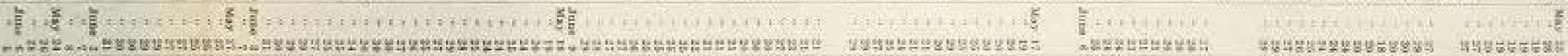

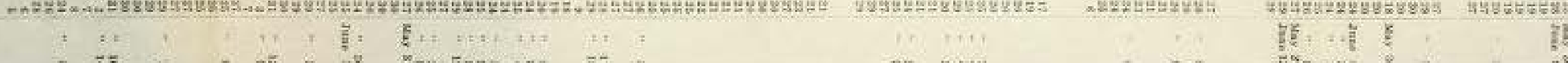

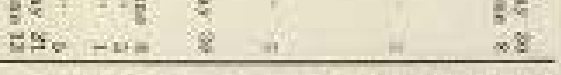
분

H:

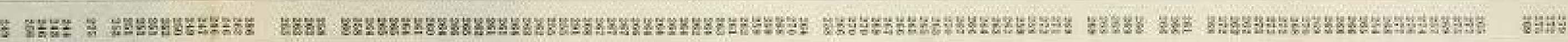

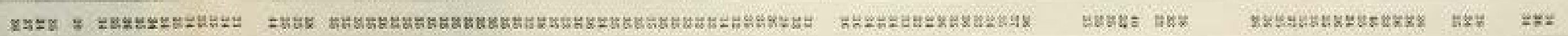

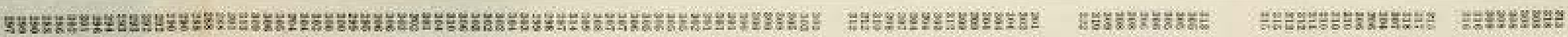

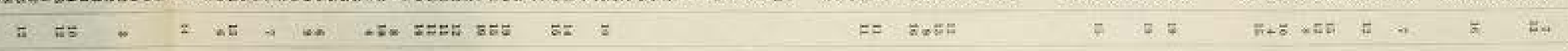

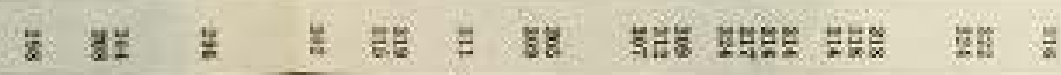

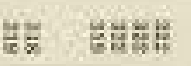
I

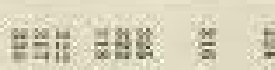
5.

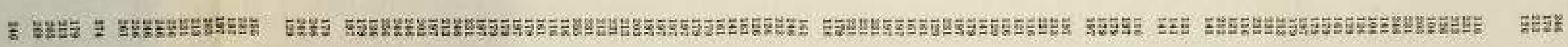


TABLE 21- Oyiposmton a sd Life Pertod of adults of the Hibernatung Genethation, 1915.

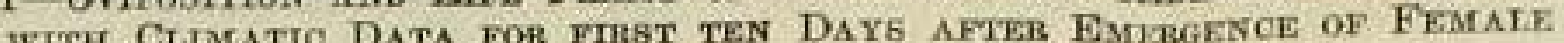

\begin{tabular}{|c|c|c|c|c|c|c|c|c|c|c|c|c|c|c|c|c|c|c|c|c|c|c|c|c|c|c|c|}
\hline \multicolumn{4}{|c|}{ Date emerged. } & \multicolumn{2}{|c|}{ Date of death. } & \multicolumn{2}{|c|}{$\begin{array}{l}\text { Life } \\
\text { period. }\end{array}$} & \multicolumn{15}{|c|}{ Number of eggs lald daily 1 to 15 days after emergence. } & \multirow[b]{2}{*}{$\frac{3}{2}$} & \multirow{2}{*}{ 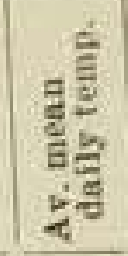 } & \multirow{2}{*}{ 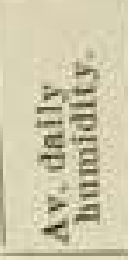 } & \multirow{2}{*}{ 总 } & \multirow{2}{*}{ 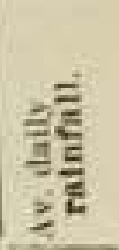 } \\
\hline Male. & & Female & & Male. & Female. & M. & F. & 1 & 2 & 3 & 4 & 5 & 6 & 7 & 8 & 9 & 10 & 11 & 12 & 13 & 14 & 15 & & & & & \\
\hline 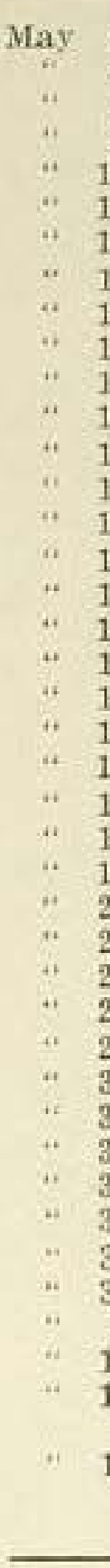 & $\begin{array}{l}3 \\
3 \\
5 \\
5 \\
17 \\
17 \\
17 \\
17 \\
17 \\
17 \\
17 \\
17 \\
17 \\
17 \\
17 \\
17 \\
18 \\
18 \\
18 \\
18 \\
18\end{array}$ & 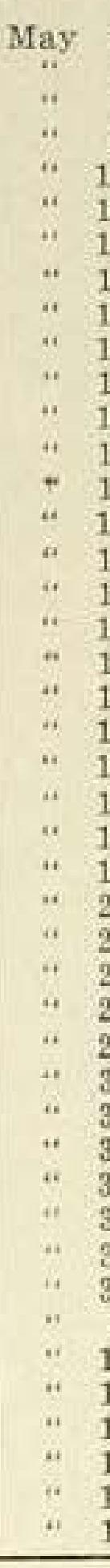 & 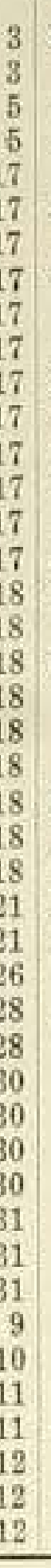 & 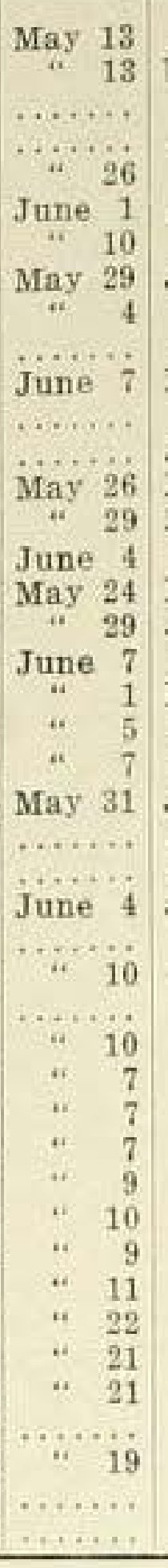 & 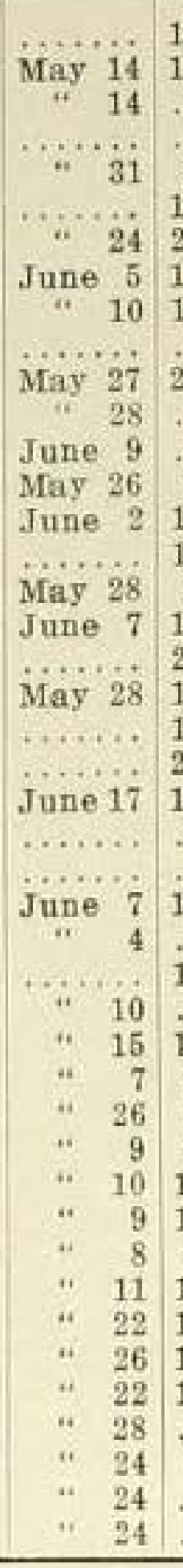 & 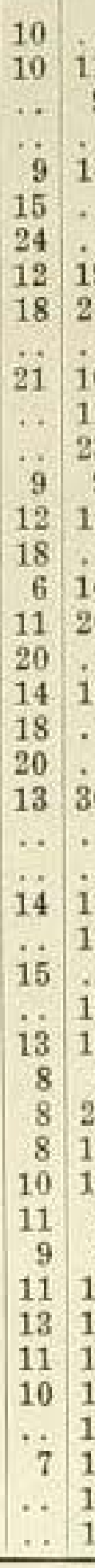 & $\left.\begin{array}{c}10 \\
\because \\
30 \\
\ldots \\
17 \\
17 \\
14 \\
\ldots \\
13 \\
18 \\
8 \\
27 \\
10 \\
12 \\
9 \\
8 \\
11 \\
13 \\
16 \\
11 \\
17 \\
12 \\
12 \\
12\end{array}\right\}$ & $\begin{array}{l}\ldots \\
\ldots \\
\ldots \\
\ldots \\
\ldots \\
32 \\
\ldots \\
\ldots \\
\ldots \\
\ldots \\
\ldots \\
. .\end{array}$ & $\begin{array}{c}\ldots \\
\ldots \\
\ldots \\
\ldots \\
\cdots \\
1 \\
3 \\
\ldots \\
32 \\
1 \\
\ldots \\
\ldots \\
\ldots\end{array}$ & $\begin{array}{r}\cdots \\
\cdots \\
\cdots \\
\ldots \\
\cdots \\
5 \\
55 \\
3 \\
1 \\
21 \\
12 \\
\ldots \\
\cdots \\
\cdots\end{array}$ & $\begin{array}{r}\ldots \\
\ldots \\
46 \\
\cdots 8 \\
31 \\
9 \\
47 \\
\ldots \\
12 \\
\ldots \\
\ldots \\
\ldots\end{array}$ & $\begin{array}{c}22 \\
\ldots \\
\ldots 8 \\
\cdots \\
\cdots \\
\cdots \\
25 \\
\cdots \\
\cdots \\
\cdots \\
\cdots \\
\cdots \\
\cdots \\
2\end{array}$ & $\begin{array}{c}\cdots \\
\cdots \\
\cdots \\
13 \\
\cdots \\
\cdots \\
\cdots \\
\cdots \\
\cdots \\
48 \\
\cdots \\
\cdots \\
\cdots \\
2\end{array}$ & $\begin{array}{l}73 \\
\ldots \\
\ldots \\
\ldots \\
\ldots \\
\cdots \\
13 \\
48 \\
\cdots \\
\cdots \\
\cdots \\
\cdots \\
\cdots \\
3\end{array}$ & \begin{tabular}{c}
$\ldots$ \\
$\ldots$ \\
$\ldots$ \\
$\ldots$ \\
$\ldots$ \\
$\cdots$ \\
$\cdots$ \\
$\ldots$ \\
$\cdots$ \\
\hdashline \\
43 \\
$\cdots$
\end{tabular} & $\begin{array}{c}\cdots \\
\cdots \\
26 \\
\cdots \\
\cdots \\
\cdots \\
\cdots \\
\ldots \\
\cdots \\
\cdots \\
3 \\
4 \\
4\end{array}$ & $\begin{array}{c}\cdots \\
\ldots \\
\cdots \\
\cdots \\
\cdots \\
\cdots \\
\cdots \\
\cdots \\
\cdots \\
\cdots \\
\cdots \\
7\end{array}$ & $\begin{array}{c}\ldots \\
\ldots \\
\ldots \\
\ldots \\
\ldots \\
\ldots \\
\ldots \\
\ldots \\
\ldots \\
30 \\
\ldots \\
\ldots\end{array}$ & $\begin{array}{c}\cdots \\
\ldots \\
\cdots \\
\cdots \\
\cdots \\
\cdots \\
\cdots \\
\cdots \\
\cdots \\
\cdots \\
\cdots \\
\ldots \\
\cdots\end{array}$ & $\begin{array}{c}\cdots \\
\ldots \\
\cdots \\
\cdots \\
\cdots \\
\cdots \\
\cdots \\
\ldots \\
\cdots \\
\cdots \\
\cdots \\
\ldots \\
\cdots\end{array}$ & $\begin{array}{c}\ldots \\
\ldots \\
\ldots \\
\ldots \\
\ldots \\
\ldots \\
\ldots \\
\cdots \\
\ldots \\
\cdots \\
\cdots \\
\cdots \\
\ldots \\
\cdots\end{array}$ & 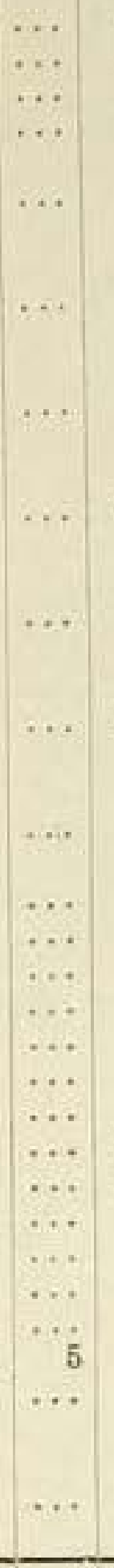 & 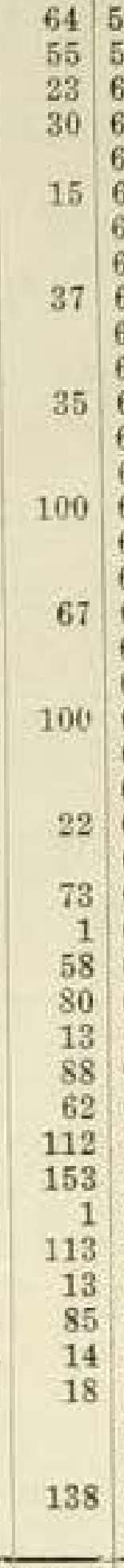 & $\begin{array}{l}59.5 \\
59.5 \\
62.0 \\
62.0 \\
60.8 \\
60.8 \\
60.8 \\
60.8 \\
60.8 \\
60.8 \\
60.8 \\
60.8 \\
60.8 \\
60.8 \\
60.8 \\
60.8 \\
61.7 \\
61.7 \\
61.7 \\
61.7 \\
61.7 \\
61.7 \\
61.7 \\
61.7 \\
61.7 \\
63.0 \\
63.0 \\
64.1 \\
67.1 \\
67.1 \\
66.9 \\
66.9 \\
66.9 \\
66.9 \\
67.0 \\
67.0 \\
67.0 \\
71.3 \\
72.4 \\
72.6 \\
72.6 \\
72.6 \\
72.6 \\
72.6 \\
\end{array}$ & $\begin{array}{l}52.5 \\
52.5 \\
51.5 \\
51.5 \\
81.1 \\
81.1 \\
81.1 \\
81.1 \\
81.1 \\
81.1 \\
81.1 \\
81.1 \\
81.1 \\
81.1 \\
81.1 \\
81.1 \\
81.3 \\
81.3 \\
81.3 \\
81.3 \\
81.3 \\
81.3 \\
81.3 \\
81.3 \\
81.3 \\
83.6 \\
83.6 \\
85.2 \\
84.3 \\
84.3 \\
82.2 \\
82.2 \\
82.2 \\
82.2 \\
81.1 \\
81.1 \\
81.1 \\
77.0 \\
77.1 \\
79.0 \\
79.0 \\
79.0 \\
79.0 \\
79.0 \\
\end{array}$ & $\begin{array}{r}25.3 \\
25.3 \\
24.2 \\
24.2 \\
24.2 \\
10.9 \\
10.9 \\
10.9 \\
10.9 \\
10.9 \\
10.9 \\
10.9 \\
10.9 \\
10.9 \\
10.9 \\
10.9 \\
10.9 \\
6.5 \\
6.5 \\
6.5 \\
6.5 \\
6.5 \\
6.5 \\
6.5 \\
6.5 \\
6.7 \\
6.7 \\
6.4 \\
8.6 \\
8.6 \\
10.6 \\
10.6 \\
10.6 \\
10.6 \\
11.6 \\
11.6 \\
17.9 \\
14.9 \\
15.2 \\
14.2 \\
14.2 \\
14.2 \\
13.1 \\
14.2 \\
\end{array}$ & $\begin{array}{l}.116 \\
.116 \\
.1305 \\
.1305 \\
.50 \\
.50 \\
.50 \\
.50 \\
.50 \\
.50 \\
.50 \\
.50 \\
.50 \\
.50 \\
.50 \\
.50 \\
.513 \\
.513 \\
.513 \\
.513 \\
.513 \\
.513 \\
.513 \\
.513 \\
.513 \\
.33 \\
.33 \\
.312 \\
.227 \\
.227 \\
.2045 \\
.2045 \\
.2045 \\
.2045 \\
.204 \\
.204 \\
.204 \\
.085 \\
.155 \\
.282 \\
.282 \\
.282 \\
.282 \\
.282 \\
\end{array}$ \\
\hline & & & & & & & otals & 91 & 126 & 186 & 356 & 249 & $128:$ & 205 & 100 & 79 & 8 & 30 & 2 & 6 & 1 & 5 & 1570 & & & & \\
\hline
\end{tabular}

SUMMABY OF TARLE 21

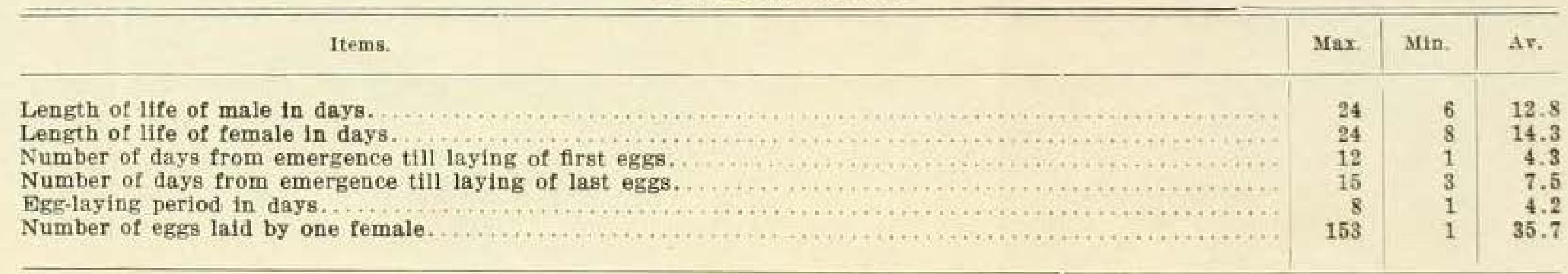


TABLe 22-Oviposition and Life Period of Hibernating Generation, OlNex, 1916,

with Chmatio Data for fikst ten Days afteil EmbBgence of Female

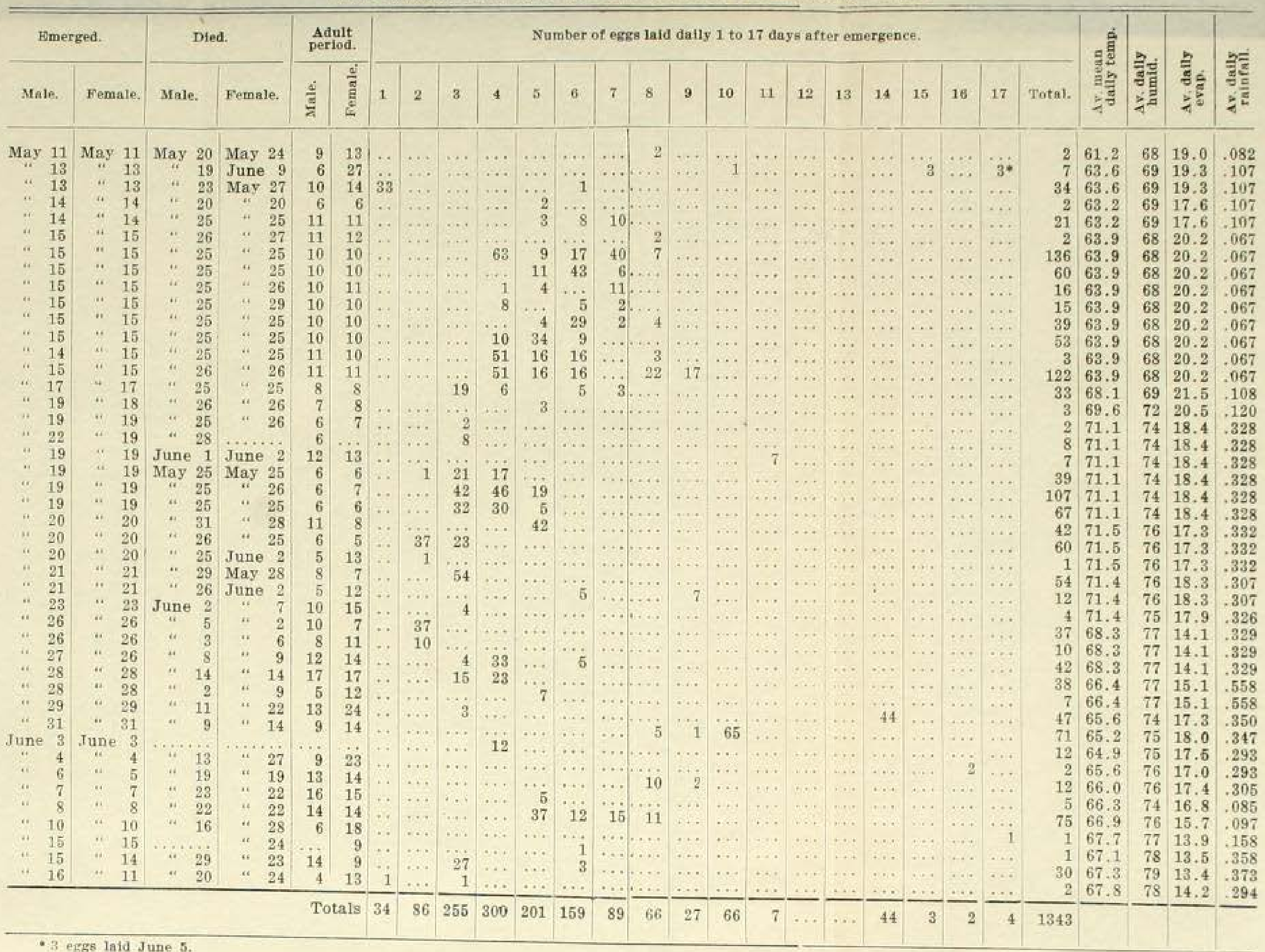

3 eggs latd June 5.

SUMMARY OF TABLE 22

Items.

Length of life of male moth in days....

Length of life of female moth in days.

Number of days from emergence to laying of first eggs.

(umger laying of last eggs.

Number of egrs laid by on

Average mean dally temperature

Average daily humidity.

Average daily evaporation in

Average daily rafneall, in inches.

Max. Min. Av.

\begin{tabular}{c|l|r|}
17 & 4 & 9.2 \\
27 & 5 & 11.9 \\
17 & 1 & 5. \\
23 & 2 & 6.9 \\
14 & 1 & 2.9 \\
136 & 1 & 30.5 \\
71.5 & 61.2 & 67.1 \\
79 & 68 & 73.2 \\
13.4 & 21.5 & 17.74 \\
.558 & .067 & .234
\end{tabular}


TABLe 23-Over and Chmatio Data for ftret ten Days aftze EMrekgence of Female

\begin{tabular}{|c|c|c|c|c|c|c|c|c|c|c|c|c|c|c|c|c|c|c|c|c|c|c|c|c|c|c|}
\hline \multicolumn{2}{|c|}{ Moths emerged. } & \multicolumn{2}{|c|}{ Moths died. } & \multicolumn{2}{|c|}{ Adult period. } & \multicolumn{17}{|c|}{ Number of eggs laid daily 1 to 16 days from date of emergence. } & \multirow{2}{*}{ 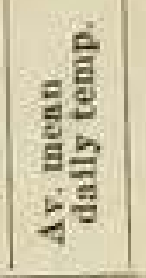 } & \multirow{2}{*}{ 送 } & \multirow{2}{*}{ 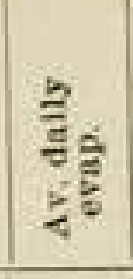 } & \multirow{2}{*}{ 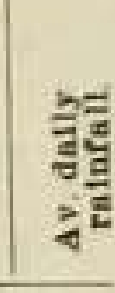 } \\
\hline Male. & Female & Male. & Female & $\frac{3}{2}$ & $\frac{\mathscr{E}}{2}$ & 1 & 2 & 3 & 4 & 5 & 6 & 7 & 8 & 9 & 10 & 11 & 12 & 13 & 14 & 15 & 16 & Total. & & & & \\
\hline $\begin{array}{cc}\text { May } & 19 \\
. & 20 \\
. & 21 \\
. & 21 \\
. & 21 \\
. & 20 \\
. & 20 \\
. & 24 \\
. & 24 \\
. & 24 \\
. & 24 \\
. & 25 \\
. & 25 \\
. & 25 \\
. & 25 \\
. " & 25 \\
. & 29 \\
. . & 28 \\
. . & 28 \\
\text { June } & 8 \\
. . & 5 \\
. . & 6 \\
. . & 9 \\
. . & 7\end{array}$ & 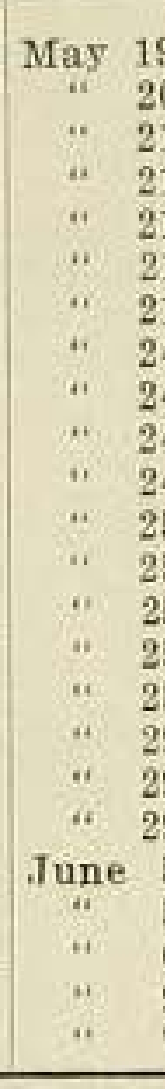 & 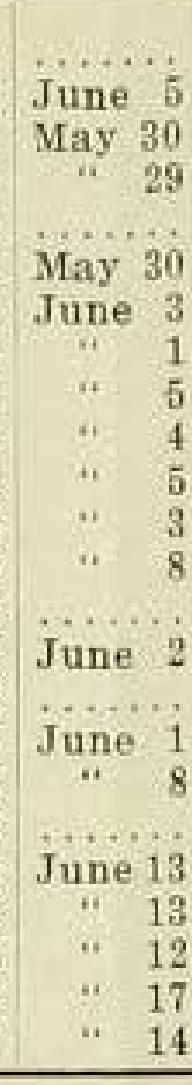 & 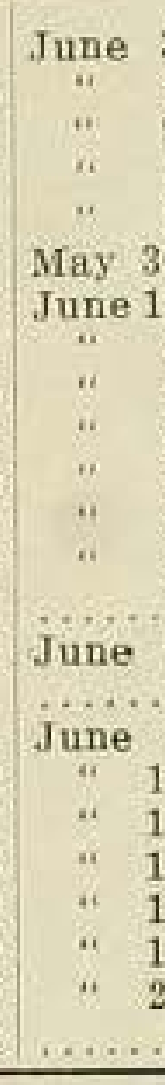 & $\begin{array}{r}16 \\
9 \\
8 \\
\ldots \ldots \\
10 \\
14 \\
8 \\
12 \\
11 \\
12 \\
9 \\
14 \\
\ldots . \\
8 \\
\ldots \\
3 \\
11 \\
\ldots \\
5 \\
8 \\
\ldots \ldots \\
\ldots . \\
7 \\
\end{array}$ & $\begin{array}{r}15 \\
16 \\
14 \\
14 \\
19 \\
9 \\
22 \\
15 \\
12 \\
15 \\
15 \\
11 \\
11 \\
\ldots 13 \\
13 \\
\ldots \\
11 \\
13 \\
15 \\
8 \\
10 \\
\ldots \ldots \\
\ldots \ldots \\
\ldots \ldots \\
\end{array}$ & $\begin{array}{l}24 \\
\ldots \\
\ldots \\
\ldots \\
\ldots \\
\ldots \\
\ldots \\
\ldots \\
\ldots \\
\ldots \\
\ldots \\
\ldots \\
\ldots \\
39 \\
\ldots \\
\end{array}$ & $\begin{array}{c}32 \\
25 \\
\ldots \\
\ldots \\
\ldots \\
\ldots \\
\ldots \\
\ldots \\
\ldots \\
\ldots \\
\ldots \\
\cdots \\
\ldots \\
\cdots \\
69 \\
\ldots \\
\ldots \\
\ldots \\
\end{array}$ & $\begin{array}{c}\cdots \\
\ldots \\
\cdots \\
\cdots \\
\cdots \\
\cdots \\
\cdots \\
38 \\
\cdots \\
\cdots \\
\cdots \\
\cdots \\
\cdots \\
28 \\
\cdots \\
\cdots \\
\end{array}$ & $\begin{array}{c}\ldots \\
\ldots \\
\ldots \\
\ldots \\
\ldots \\
\ldots \\
\ldots \\
\cdots \\
21 \\
48 \\
18 \\
7 \\
\ldots \\
\cdots \\
\ldots \\
\cdots \\
56 \\
\cdots \\
\ldots \\
\end{array}$ & 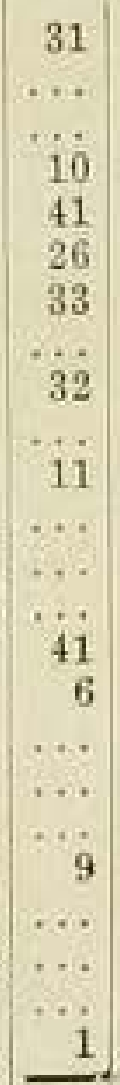 & 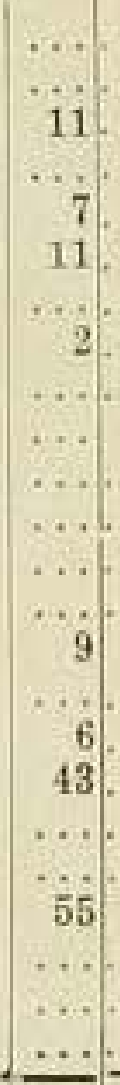 & 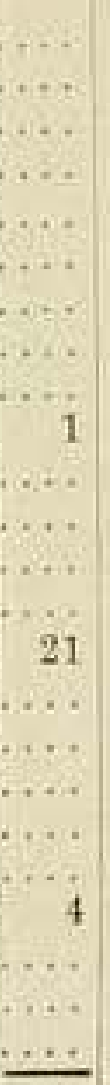 & $\begin{array}{r}\ldots \\
\ldots \\
2 \\
1 \\
\cdots 1 \\
\cdots \\
\cdots \\
\cdots \\
16 \\
\cdots \\
\cdots \\
\cdots \\
\cdots \\
\cdots \\
\cdots \\
16 \\
\cdots \\
\end{array}$ & $\begin{array}{c}9 \\
\ldots \\
\ldots \\
\ldots \\
\ldots \\
\ldots \\
\ldots \\
\ldots \\
\ldots \\
\ldots \\
\ldots \\
\ldots \\
27 \\
\ldots \\
\ldots \\
\ldots \\
\ldots \\
\end{array}$ & $\begin{array}{r}\quad 5 \\
3 \\
\ldots \\
\ldots \\
\ldots \\
\ldots \\
\ldots \\
\ldots \\
\ldots \\
\ldots \\
\ldots \\
\ldots \\
9 \\
4 \\
\ldots \\
\ldots \\
6 \\
\ldots \\
\end{array}$ & $\begin{array}{c}\ldots \\
\ldots \\
\ldots \\
\ldots \\
\ldots \\
\ldots \\
\ldots \\
\cdots \\
\ldots \\
\cdots \\
\cdots \\
\cdots \\
\cdots \\
\cdots \\
\cdots \\
50 \\
\end{array}$ & $\begin{array}{c}\ldots \\
\ldots \\
\ldots \\
\ldots \\
\ldots \\
\ldots \\
\ldots \\
\ldots \\
\ldots \\
\ldots \\
\ldots \\
\ldots \\
\ldots \\
\ldots \\
\ldots \\
\ldots \\
\cdots \\
13 \\
\end{array}$ & $\begin{array}{c}\ldots \\
\ldots \\
\ldots \\
\ldots \\
\ldots \\
\ldots \\
\ldots \\
\ldots \\
\ldots \\
\ldots \\
\ldots \\
\ldots \\
\ldots \\
\ldots \\
\ldots \\
\ldots \\
\ldots \\
\end{array}$ & 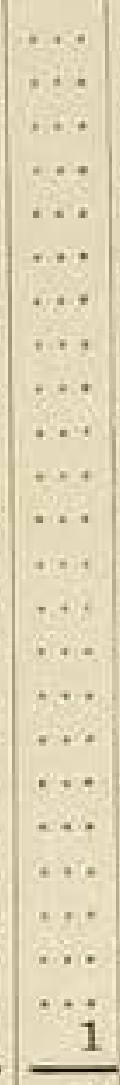 & 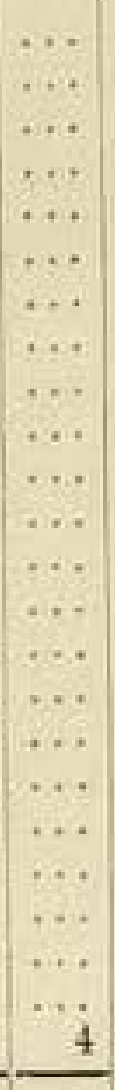 & $\begin{array}{c}\ldots \\
\ldots \\
\ldots \\
\ldots \\
\ldots \\
\ldots \\
\ldots \\
\ldots \\
\ldots \\
\ldots \\
\ldots \\
\ldots \\
\ldots \\
\ldots \\
\ldots \\
\ldots \\
\ldots \\
\ldots \\
\end{array}$ & $\begin{array}{r}96 \\
35 \\
14 \\
12 \\
49 \\
37 \\
54 \\
2 \\
32 \\
1 \\
11 \\
75 \\
48 \\
18 \\
78 \\
6 \\
15 \\
70 \\
4 \\
9 \\
156 \\
95 \\
22 \\
79 \\
\end{array}$ & $\begin{array}{l}62.1 \\
61.4 \\
61.4 \\
61.4 \\
61.4 \\
61.4 \\
61.4 \\
63.8 \\
63.8 \\
63.8 \\
63.8 \\
64.2 \\
64.2 \\
64.2 \\
64.2 \\
64.2 \\
67.8 \\
67.8 \\
67.8 \\
68.5 \\
68.5 \\
66.2 \\
64.8 \\
65.2 \\
\end{array}$ & $\begin{array}{l}72.3 \\
73.3 \\
73.9 \\
73.9 \\
73.9 \\
73.9 \\
73.9 \\
77.3 \\
77.3 \\
77.3 \\
77.3 \\
78.5 \\
78.5 \\
78.5 \\
78.5 \\
78.5 \\
76.2 \\
76.2 \\
76.2 \\
75.4 \\
75.4 \\
75.4 \\
75.1 \\
76.0 \\
\end{array}$ & $\begin{array}{r}11.3 \\
9.9 \\
11.4 \\
11.4 \\
11.4 \\
11.4 \\
11.4 \\
11.8 \\
11.8 \\
11.8 \\
11.8 \\
11.8 \\
11.8 \\
11.8 \\
11.8 \\
11.8 \\
12.2 \\
12.2 \\
12.2 \\
14.8 \\
14.8 \\
14.7 \\
16.0 \\
14.3 \\
\end{array}$ & $\begin{array}{l}.209 \\
.210 \\
.278 \\
.278 \\
.278 \\
.278 \\
.278 \\
.457 \\
.457 \\
.457 \\
.457 \\
.457 \\
.457 \\
.457 \\
.457 \\
.457 \\
.528 \\
.528 \\
.528 \\
.188 \\
.188 \\
.17 \\
.17 \\
.17 \\
\end{array}$ \\
\hline \multicolumn{6}{|c|}{ Totals } & 63 & 126 & 66 & 150 & 250 & 144 & 26 & 54 & 37 & 27 & 50 & 13 & $\ldots$ & 1 & 4 & 7 & 1018 & & & & \\
\hline
\end{tabular}

SUMMARY OF TABLE 23

\begin{tabular}{|c|c|c|c|}
\hline Items. & Max. & Min. & Av. \\
\hline 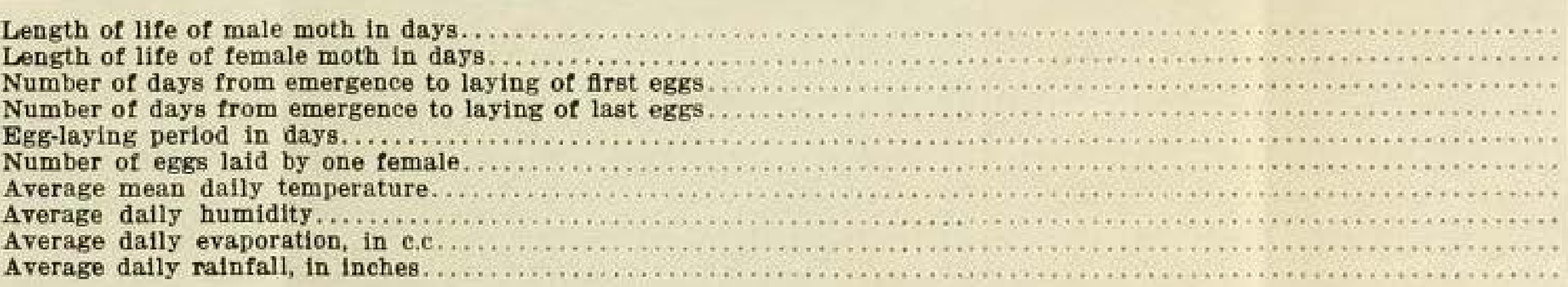 & $\begin{array}{l}16 \\
22 \\
10 \\
16 \\
12 \\
156 \\
68.5 \\
78.5 \\
16.0 \\
.528\end{array}$ & $\begin{array}{c}3 \\
8 \\
1 \\
4 \\
1 \\
1 \\
61.4 \\
72.3 \\
9.9 \\
.17\end{array}$ & $\begin{array}{r}9.2 \\
13.5 \\
4.8 \\
7.8 \\
4.0 \\
42.4 \\
64.3 \\
76.0 \\
12.3 \\
.33\end{array}$ \\
\hline
\end{tabular}




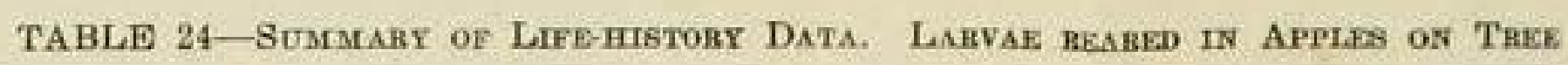

\begin{tabular}{|c|c|c|c|c|c|c|c|c|c|c|c|c|c|c|c|c|c|c|c|c|}
\hline \multirow{11}{*}{ 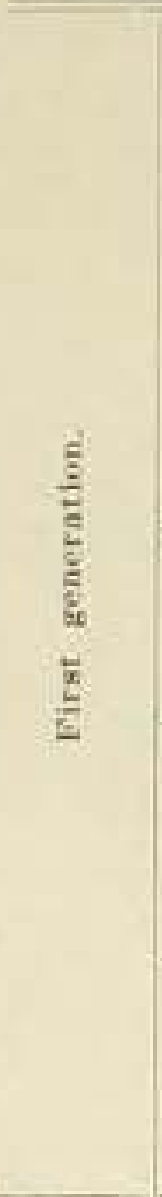 } & \multirow{3}{*}{ Year. } & \multirow{3}{*}{ Sex. } & \multicolumn{2}{|c|}{ Egg. } & \multicolumn{2}{|c|}{ Larva in fruit. } & \multicolumn{2}{|c|}{ Larva in cocoon. } & \multicolumn{2}{|c|}{ Larva. } & \multicolumn{2}{|c|}{ Pupa. } & \multicolumn{4}{|c|}{ Egg, larva, and pupa. } & \multicolumn{2}{|c|}{ Aduit. } & \multicolumn{2}{|c|}{$\begin{array}{l}\text { Total life } \\
\text { Deriod. }\end{array}$} \\
\hline & & & \multirow[b]{2}{*}{ No. } & \multirow{2}{*}{$\begin{array}{c}\text { Period } \\
\text { in } \\
\text { days. }\end{array}$} & \multirow[b]{2}{*}{ No. } & \multirow{2}{*}{$\begin{array}{l}\text { Period } \\
\text { in } \\
\text { days. }\end{array}$} & \multirow[b]{2}{*}{ No. } & \multirow{2}{*}{$\begin{array}{l}\text { Period } \\
\text { In } \\
\text { days. }\end{array}$} & \multirow[b]{2}{*}{ No. } & \multirow{2}{*}{$\begin{array}{l}\text { Period } \\
\text { in } \\
\text { days. }\end{array}$} & \multirow[b]{2}{*}{ No. } & \multirow{2}{*}{$\begin{array}{l}\text { Perlod } \\
\text { in } \\
\text { days. }\end{array}$} & \multirow[b]{2}{*}{ No. } & \multirow{2}{*}{$\begin{array}{l}\text { Period } \\
\text { In } \\
\text { days. }\end{array}$} & \multicolumn{2}{|c|}{$\begin{array}{l}\text { Total effective } \\
\text { day-degrees. }\end{array}$} & \multirow{2}{*}{ No. } & \multirow{2}{*}{$\begin{array}{c}\text { Period } \\
\text { in } \\
\text { days. }\end{array}$} & \multirow[b]{2}{*}{ No. } & \\
\hline & & & & & & & & & & & & & & & $\begin{array}{c}\text { Sum } \\
\text { for } \\
\text { stages. }\end{array}$ & $\left(\begin{array}{l}50+)- \\
2(86+)\end{array}\right.$ & & & & $\begin{array}{c}\text { Perlod } \\
\text { in } \\
\text { dayse }\end{array}$ \\
\hline & 1915 & $\begin{array}{l}\text { Male } \\
\text { Female }\end{array}$ & $\begin{array}{l}28 \\
46\end{array}$ & $\begin{array}{l}9.6 \\
9.7\end{array}$ & $\begin{array}{l}16 \\
36\end{array}$ & $\begin{array}{l}28.3 \\
28.9 \\
\end{array}$ & $\begin{array}{l}16 \\
31\end{array}$ & $\begin{array}{l}4.3 \\
4.4 \\
\end{array}$ & $\begin{array}{l}28 \\
44 \\
\end{array}$ & $\begin{array}{l}31.9 \\
34.6 \\
\end{array}$ & $\begin{array}{l}28 \\
44\end{array}$ & $\begin{array}{l}10.7 \\
10.5\end{array}$ & $\begin{array}{l}28 \\
46\end{array}$ & $\begin{array}{l}51.9 \\
54.3\end{array}$ & $\begin{array}{l}1096 \\
1154 \\
\end{array}$ & $\begin{array}{l}1095 \\
1154 \\
\end{array}$ & & & & \\
\hline & & Male and female & 74 & 9.7 & 52 & 28.7 & 47 & 4,4 & 72 & 33.5 & 72 & 10.6 & 74 & 53.4 & 1132 & 1131 & & & & \\
\hline & 1916 & $\begin{array}{l}\text { Male } \\
\text { Female }\end{array}$ & $\begin{array}{l}31 \\
34\end{array}$ & $\begin{array}{l}7.8 \\
7.8\end{array}$ & $\begin{array}{l}28 \\
30\end{array}$ & $\begin{array}{l}31.2 \\
31.0 \\
\end{array}$ & $\begin{array}{l}27 \\
30\end{array}$ & $\begin{array}{l}3.3 \\
3.6 \\
\end{array}$ & $\begin{array}{l}28 \\
32\end{array}$ & $\begin{array}{l}34.6 \\
35.1\end{array}$ & $\begin{array}{l}28 \\
32\end{array}$ & $\begin{array}{r}10.1 \\
9.8 \\
\end{array}$ & $\begin{array}{l}31 \\
34\end{array}$ & $\begin{array}{l}52.1 \\
52.7 \\
\end{array}$ & $\begin{array}{l}1127 \\
1132\end{array}$ & $\begin{array}{l}1125 \\
1131 \\
\end{array}$ & $\begin{array}{l}24 \\
29 \\
\end{array}$ & $\begin{array}{l}5.1 \\
6.9\end{array}$ & $\begin{array}{l}24 \\
29\end{array}$ & $\begin{array}{l}57.4 \\
59.8\end{array}$ \\
\hline & & $\overline{\text { Male and female }}$ & 65 & 7.8 & 58 & 31.1 & 57 & 3.5 & 60 & 34.9 & 60 & 10.0 & 65 & 52.4 & 1129 & 1128 & 53 & 6.1 & 53 & 58.7 \\
\hline & 1917 & \begin{tabular}{|l} 
Male \\
Female \\
Undetermined \\
\end{tabular} & $\begin{array}{r}44 \\
51 \\
9 \\
\end{array}$ & $\begin{array}{l}9.2 \\
9.3 \\
9.8 \\
\end{array}$ & $\begin{array}{r}43 \\
48 \\
8 \\
\end{array}$ & $\begin{array}{l}25.1 \\
26.1 \\
26.4 \\
\end{array}$ & $\begin{array}{r}41 \\
46 \\
8 \\
\end{array}$ & $\begin{array}{l}4.7 \\
4.4 \\
4.3 \\
\end{array}$ & $\begin{array}{r}41 \\
46 \\
8 \\
\end{array}$ & $\begin{array}{l}29.6 \\
30.5 \\
30.6 \\
\end{array}$ & $\begin{array}{r}41 \\
47 \\
9 \\
\end{array}$ & $\begin{array}{l}10.9 \\
10.9 \\
11.0 \\
\end{array}$ & $\begin{array}{r}44 \\
51 \\
9 \\
\end{array}$ & $\begin{array}{l}49.8 \\
50.5 \\
51.6 \\
\end{array}$ & $\begin{array}{l}1095 \\
1104 \\
1095 \\
\end{array}$ & $\begin{array}{l}1107 \\
1116 \\
1105 \\
\end{array}$ & & & & \\
\hline & & Male and female & 104 & 9.3 & 99 & 25.7 & 95 & 4.5 & 95 & 30.1 & 97 & 10.9 & 104 & 50.3 & 1100 & 1111 & & & & \\
\hline & $\begin{array}{l}1915, \\
1916,\end{array}$ & $\begin{array}{l}\text { Male } \\
\text { Female } \\
\text { Undetermined } \\
\end{array}$ & $\begin{array}{r}103 \\
131 \\
9 \\
\end{array}$ & $\begin{array}{l}8.9 \\
9.0 \\
9.8 \\
\end{array}$ & $\begin{array}{r}87 \\
114 \\
8 \\
\end{array}$ & $\begin{array}{l}27.6 \\
28.3 \\
26.4 \\
\end{array}$ & $\begin{array}{r}84 \\
107 \\
8 \\
\end{array}$ & $\begin{array}{l}4.2 \\
4.2 \\
4.5 \\
\end{array}$ & $\begin{array}{r}97 \\
122 \\
8 \\
\end{array}$ & $\begin{array}{l}31.7 \\
33.2 \\
30.6 \\
\end{array}$ & $\begin{array}{r}97 \\
123 \\
9 \\
\end{array}$ & $\begin{array}{l}10.6 \\
10.5 \\
11.0 \\
\end{array}$ & $\begin{array}{r}103 \\
131 \\
9 \\
\end{array}$ & $\begin{array}{l}51.1 \\
52.4 \\
51.6 \\
\end{array}$ & $\begin{array}{l}1104 \\
1128 \\
1095 \\
\end{array}$ & $\begin{array}{l}1109 \\
1133 \\
1105 \\
\end{array}$ & $\begin{array}{l}24 \\
29\end{array}$ & $\begin{array}{l}5.1 \\
6.9\end{array}$ & $\begin{array}{l}24 \\
29\end{array}$ & $\begin{array}{l}57.4 \\
59.8\end{array}$ \\
\hline & & Male and female & 243 & 9.0 & 209 & 27.9 & 199 & 4.2 & 227 & 32.5 & 229 & 10.6 & 243 & 51.8 & 1118 & 1122 & 53 & 6.1 & 53 & 58.7 \\
\hline & 1915 & $\begin{array}{l}\text { Male } \\
\text { Female } \\
\text { Undetermined } \\
\end{array}$ & $\begin{array}{l}5 \\
8 \\
1\end{array}$ & $\begin{array}{l}6.8 \\
6.5 \\
9.0 \\
\end{array}$ & $\begin{array}{l}3 \\
8 \\
1 \\
\end{array}$ & $\begin{array}{l}25.3 \\
24.8 \\
20.0 \\
\end{array}$ & $\begin{array}{l}3 \\
8 \\
1 \\
\end{array}$ & $\begin{array}{l}4.0 \\
4.1 \\
5.0 \\
\end{array}$ & $\begin{array}{l}3 \\
8 \\
1 \\
\end{array}$ & $\begin{array}{l}29.3 \\
29.0 \\
25.0 \\
\end{array}$ & $\begin{array}{l}3 \\
8 \\
0 \\
\end{array}$ & $\begin{array}{l}15.3 \\
13.6\end{array}$ & $\begin{array}{l}5 \\
8 \\
0 \\
\end{array}$ & $\begin{array}{l}49.0 \\
49.0\end{array}$ & $\begin{array}{l}1106 \\
1100\end{array}$ & $\begin{array}{l}1106 \\
1099\end{array}$ & & & & \\
\hline 년 & & Male and female & 14 & 6.8 & 12 & 24.5 & 12 & 4.2 & 12 & 28.7 & 11 & 14.1 & 13 & 49.0 & 1102 & 1102 & & & & \\
\hline 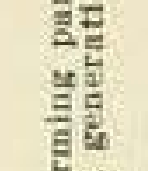 & 1916 & $\begin{array}{l}\text { Male } \\
\text { Female } \\
\text { Undetermined }\end{array}$ & $\begin{array}{r}36 \\
54 \\
2 \\
\end{array}$ & $\begin{array}{l}5.8 \\
6.0 \\
6.5\end{array}$ & $\begin{array}{r}33 \\
51 \\
2 \\
\end{array}$ & $\begin{array}{l}21.6 \\
22.9 \\
20.5 \\
\end{array}$ & $\begin{array}{r}32 \\
51 \\
2 \\
\end{array}$ & $\begin{array}{l}4.2 \\
4.3 \\
3.0 \\
\end{array}$ & $\begin{array}{r}33 \\
51 \\
2\end{array}$ & $\begin{array}{l}26.0 \\
27.2 \\
23.5 \\
\end{array}$ & $\begin{array}{r}33 \\
51 \\
2\end{array}$ & $\begin{array}{l}11.8 \\
12.0 \\
10.5 \\
\end{array}$ & $\begin{array}{r}36 \\
54 \\
2 \\
\end{array}$ & $\begin{array}{l}43.0 \\
44.9 \\
40.5 \\
\end{array}$ & $\begin{array}{l}1084 \\
1119 \\
1071\end{array}$ & $\begin{array}{l}1099 \\
1133 \\
1088 \\
\end{array}$ & $\begin{array}{l}25 \\
38\end{array}$ & $\begin{array}{r}9.7 \\
10.2\end{array}$ & $\begin{array}{l}25 \\
38\end{array}$ & $\begin{array}{l}51.3 \\
55.1\end{array}$ \\
\hline 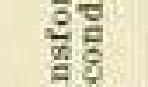 & & Male and female & 92 & 6.0 & 86 & 22.3 & 85 & 4,2 & 86 & 26.4 & 86 & 11.9 & 92 & 44.1 & 1106 & 1118 & 63 & 10.0 & 63 & 53.6 \\
\hline . & $\begin{array}{l}1915, \\
1916\end{array}$ & \begin{tabular}{|l|} 
Male \\
Female \\
Undetermined \\
\end{tabular} & $\begin{array}{r}41 \\
62 \\
3 \\
\end{array}$ & $\begin{array}{l}5.9 \\
6.1 \\
7.3 \\
\end{array}$ & $\begin{array}{r}36 \\
59 \\
3 \\
\end{array}$ & $\begin{array}{l}21.9 \\
23.1 \\
20.3 \\
\end{array}$ & $\begin{array}{r}35 \\
59 \\
3 \\
\end{array}$ & $\begin{array}{l}4.2 \\
4.3 \\
3.7\end{array}$ & $\begin{array}{r}36 \\
59 \\
3 \\
\end{array}$ & $\begin{array}{l}26.3 \\
27.4 \\
24.0 \\
\end{array}$ & $\begin{array}{r}36 \\
59 \\
2 \\
\end{array}$ & $\begin{array}{l}12.1 \\
12.2 \\
10.5 \\
\end{array}$ & $\begin{array}{r}41 \\
62 \\
2 \\
\end{array}$ & $\begin{array}{l}43.7 \\
45.4 \\
40.5 \\
\end{array}$ & $\begin{array}{l}1087 \\
1117 \\
1071 \\
\end{array}$ & $\begin{array}{l}1100 \\
1129 \\
1088 \\
\end{array}$ & $\begin{array}{l}25 \\
38\end{array}$ & $\begin{array}{r}9.7 \\
10.2\end{array}$ & $\begin{array}{l}25 \\
38\end{array}$ & $\begin{array}{l}51.3 \\
55.1\end{array}$ \\
\hline & & Male and female & 106 & 6.0 & 98 & 22.6 & 97 & 4.2 & 98 & 26.8 & 97 & 12.1 & 105 & 44.7 & 1104 & 1119 & 63 & 10.0 & 63 & 53.6 \\
\hline$\stackrel{2}{2}$ & $1915-16$ & $\begin{array}{l}\text { Male } \\
\text { Female } \\
\text { Undetermined } \\
\end{array}$ & $\begin{array}{l}43 \\
46 \\
22 \\
\end{array}$ & $\begin{array}{l}7.1 \\
7.1 \\
8.8 \\
\end{array}$ & $\begin{array}{l}42 \\
43 \\
16 \\
\end{array}$ & $\begin{array}{l}32.7 \\
33.9 \\
31.1 \\
\end{array}$ & $\begin{array}{l}41 \\
41 \\
16\end{array}$ & $\begin{array}{l}233 \\
237 \\
227 \\
\end{array}$ & $\begin{array}{l}41 \\
42 \\
22\end{array}$ & $\begin{array}{l}266 \\
272 \\
255 \\
\end{array}$ & $\begin{array}{r}42 \\
44 \\
8 \\
\end{array}$ & $\begin{array}{l}26.5 \\
25.8 \\
26.6 \\
\end{array}$ & $\begin{array}{r}43 \\
46 \\
8 \\
\end{array}$ & $\begin{array}{l}299 \\
304 \\
289 \\
\end{array}$ & & & $\begin{array}{r}6 \\
35\end{array}$ & $\begin{array}{l}7.5 \\
9.1\end{array}$ & $\begin{array}{r}6 \\
35\end{array}$ & 306 \\
\hline & & Male and female & 111 & 7.4 & 101 & 33.0 & 98 & 234 & 105 & 266 & 94 & 26.2 & 97 & 301 & & & 41 & 8.9 & 41 & 310 \\
\hline 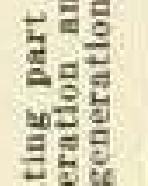 & $1916-17$ & \begin{tabular}{|l|} 
Male \\
Female \\
Undetermined \\
\end{tabular} & $\begin{array}{r}57 \\
66 \\
8 \\
\end{array}$ & $\begin{array}{l}6.1 \\
5.9 \\
6.1 \\
\end{array}$ & $\begin{array}{r}56 \\
62 \\
5 \\
\end{array}$ & $\begin{array}{l}26.4 \\
26.3 \\
25.4 \\
\end{array}$ & $\begin{array}{r}54 \\
54 \\
5 \\
\end{array}$ & $\begin{array}{l}236 \\
240 \\
244 \\
\end{array}$ & $\begin{array}{r}55 \\
56 \\
7 \\
\end{array}$ & $\begin{array}{l}262 \\
266 \\
267 \\
\end{array}$ & $\begin{array}{r}52 \\
52 \\
2 \\
\end{array}$ & $\begin{array}{r}35.0 \\
35.0 \\
34.5 \\
\end{array}$ & $\begin{array}{r}54 \\
61 \\
3 \\
\end{array}$ & $\begin{array}{l}303 \\
306 \\
307 \\
\end{array}$ & & & $\begin{array}{l}18 \\
25\end{array}$ & $\begin{array}{r}8.7 \\
12.8\end{array}$ & $\begin{array}{l}18 \\
25\end{array}$ & $\begin{array}{l}314 \\
318\end{array}$ \\
\hline 茛跑 & & Male and female & 131 & 6.0 & 123 & 26.3 & 113 & 238 & 118 & 264 & 106 & 34.3 & 118 & 305 & & & 43 & 11.1 & 43 & 317 \\
\hline 语 & $\begin{array}{c}1915-16 \\
\text { and } \\
1916-17\end{array}$ & $\begin{array}{l}\text { Male } \\
\text { Female } \\
\text { Undetermined } \\
\end{array}$ & $\begin{array}{r}100 \\
112 \\
30 \\
\end{array}$ & $\begin{array}{l}6.5 \\
6.4 \\
8.1 \\
\end{array}$ & $\begin{array}{r}98 \\
105 \\
21 \\
\end{array}$ & $\begin{array}{l}29.1 \\
29.4 \\
29.7 \\
\end{array}$ & $\begin{array}{l}95 \\
95 \\
21 \\
\end{array}$ & $\begin{array}{l}235 \\
239 \\
231 \\
\end{array}$ & $\begin{array}{l}96 \\
98 \\
29 \\
\end{array}$ & $\begin{array}{l}264 \\
269 \\
258 \\
\end{array}$ & $\begin{array}{l}94 \\
96 \\
10 \\
\end{array}$ & $\begin{array}{l}31.2 \\
30.8 \\
28.2\end{array}$ & $\begin{array}{r}97 \\
107 \\
11 \\
\end{array}$ & $\begin{array}{l}301 \\
305 \\
294 \\
\end{array}$ & & & $\frac{24}{60}$ & $\begin{array}{r}8.4 \\
10.6\end{array}$ & $\begin{array}{l}24 \\
60\end{array}$ & $\begin{array}{l}312 \\
314\end{array}$ \\
\hline & & Male and female & 242 & 6.7 & 224 & 29.3 & 211 & 236 & 223 & 265 & 200 & 30.9 & 215 & 303 & & & 84 & 10.0 & 84 & 313 \\
\hline
\end{tabular}


Table 25-Summary of Life-history Data. First Genfration Larvae rearfd in Picked Apples

\begin{tabular}{|c|c|c|c|c|c|c|c|c|c|c|c|c|c|c|c|c|c|c|c|}
\hline \multirow{2}{*}{ Year. } & \multirow{2}{*}{ Ser. } & \multicolumn{2}{|c|}{ Egg. } & \multicolumn{2}{|c|}{ Larva in fruit } & \multicolumn{2}{|c|}{ Larva in cocoon } & \multicolumn{2}{|c|}{ Larva. } & \multicolumn{2}{|c|}{ Pupa. } & \multicolumn{4}{|c|}{$\frac{\text { Egg, larva, and pupa. }}{\mid \begin{array}{l}\text { Total effective } \\
\text { day-degrees. }\end{array}}$} & \multicolumn{2}{|c|}{ Adult. } & \multicolumn{2}{|c|}{ Total life period } \\
\hline & & No. & $\begin{array}{c}\text { Perlod } \\
\text { In } \\
\text { days. }\end{array}$ & No. & $\begin{array}{c}\text { Perlod } \\
\text { in } \\
\text { days. }\end{array}$ & No. & $\begin{array}{c}\text { Perlod } \\
\text { in } \\
\text { days. }\end{array}$ & No. & $\begin{array}{c}\text { Period } \\
\text { In } \\
\text { days. }\end{array}$ & No. & $\begin{array}{l}\text { Perlod } \\
\text { in } \\
\text { days. }\end{array}$ & No. & $\begin{array}{c}\text { Perlod } \\
\text { in } \\
\text { days. }\end{array}$ & $\begin{array}{c}\text { Sum } \\
\text { for } \\
\text { stages }\end{array}$ & 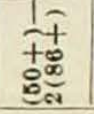 & No. & $\begin{array}{c}\text { Perlod } \\
\text { in } \\
\text { days. }\end{array}$ & No. & $\begin{array}{c}\text { Period } \\
\text { in } \\
\text { days. }\end{array}$ \\
\hline \multirow[t]{2}{*}{1915} & $\begin{array}{l}\text { Male } \\
\text { Female }\end{array}$ & $\begin{array}{l}14 \\
17\end{array}$ & $\begin{array}{l}8.0 \\
7.8\end{array}$ & $\begin{array}{l}7 \\
9\end{array}$ & $\begin{array}{l}24.9 \\
22.4\end{array}$ & $\begin{array}{l}7 \\
9\end{array}$ & $\begin{array}{l}3.7 \\
3.6\end{array}$ & $\begin{array}{l}14 \\
17\end{array}$ & $\begin{array}{l}27.2 \\
27.1\end{array}$ & $\begin{array}{l}14 \\
17\end{array}$ & $\begin{array}{l}10.6 \\
10.8\end{array}$ & $\begin{array}{l}14 \\
17\end{array}$ & $\begin{array}{l}45.9 \\
45.7\end{array}$ & $\begin{array}{l}1002 \\
1013\end{array}$ & $\begin{array}{r}999 \\
1012\end{array}$ & & & & \\
\hline & Male and female & 31 & 7.9 & 16 & 23.5 & 6 & 3.6 & 31 & 27.1 & 31 & 10.7 & 31 & 45.8 & 1008 & 1006 & & & & \\
\hline \multirow[t]{2}{*}{1916} & $\begin{array}{l}\text { Male } \\
\text { Female } \\
\end{array}$ & $\begin{array}{l}37 \\
50 \\
\end{array}$ & $\begin{array}{l}9.7 \\
9.4 \\
\end{array}$ & $\begin{array}{l}32 \\
50\end{array}$ & $\begin{array}{l}21.2 \\
20.4 \\
\end{array}$ & $\begin{array}{l}32 \\
49\end{array}$ & $\begin{array}{l}4.6 \\
4.6 \\
\end{array}$ & $\begin{array}{l}33 \\
49 \\
\end{array}$ & $\begin{array}{l}26.0 \\
24.8 \\
\end{array}$ & $\begin{array}{l}33 \\
49 \\
\end{array}$ & $\begin{array}{l}10.1 \\
10.0 \\
\end{array}$ & $\begin{array}{l}37 \\
50 \\
\end{array}$ & $\begin{array}{l}46.0 \\
44.4 \\
\end{array}$ & $\begin{array}{l}1018 \\
1005 \\
\end{array}$ & $\begin{array}{l}1018 \\
1006 \\
\end{array}$ & $\begin{array}{l}30 \\
44 \\
\end{array}$ & $\begin{array}{l}5.3 \\
6.4 \\
\end{array}$ & $\begin{array}{l}30 \\
44\end{array}$ & $\begin{array}{l}51.6 \\
50.5 \\
\end{array}$ \\
\hline & Male and female & 87 & 9.5 & 82 & 20.7 & 81 & 4.6 & 82 & 25.3 & 82 & 10.1 & 87 & 45.1 & 1011 & 1011 & 74 & 5.9 & 74 & 50.9 \\
\hline \multirow{2}{*}{$\begin{array}{l}1915 \\
\text { and } \\
1916\end{array}$} & $\begin{array}{l}\text { Male } \\
\text { Female }\end{array}$ & $\begin{array}{l}51 \\
67 \\
\end{array}$ & $\begin{array}{l}9.2 \\
9.0 \\
\end{array}$ & $\begin{array}{l}39 \\
59\end{array}$ & $\begin{array}{l}21.9 \\
20.7\end{array}$ & $\begin{array}{l}39 \\
58\end{array}$ & $\begin{array}{l}4.4 \\
4.4 \\
\end{array}$ & $\begin{array}{l}47 \\
66\end{array}$ & $\begin{array}{l}26.4 \\
25.4\end{array}$ & $\begin{array}{l}47 \\
66\end{array}$ & $\begin{array}{l}10.2 \\
10.2\end{array}$ & $\begin{array}{l}51 \\
67\end{array}$ & $\begin{array}{l}45.9 \\
44.7\end{array}$ & $\begin{array}{l}1014 \\
1007\end{array}$ & $\begin{array}{l}1013 \\
1008 \\
\end{array}$ & $\begin{array}{l}30 \\
44\end{array}$ & $\begin{array}{l}5.3 \\
6.4\end{array}$ & $\begin{array}{l}30 \\
44\end{array}$ & $\begin{array}{l}51.6 \\
50.5\end{array}$ \\
\hline & Male and female & 118 & 9.1 & 98 & 21.2 & 97 & 4.4 & 113 & 25.8 & 113 & 10.2 & 118 & 45.2 & 1010 & 1010 & 74 & 5.9 & 74 & 50.9 \\
\hline
\end{tabular}


11. Taking 50 as the zero of development for all three stages and disregarding the slight differences in the units due to the use of 88,85 , and 87 as the degree of the maximum rate of development of the egg, larva, and pupa, respectively, we have for the equation of development of the three stages $\mathrm{PE}=1101$.

Since the larval period is about equal to the egg and pupal periods combined, the use of 86 degrees as the degree of maximum rate of development for all three stages will give about the same results and will give units of the same value for all three stages.

\section{Observations on the Life History of the Codling-moth IN 1915, 1916, AND 1917}

Continuous observations were made on the life history of individuals throughout the seasons of 1915,1916 , and 1917 . The methods employed in making these observations are described on page 228 of this article.

The following tables give the principal facts as to length of the periods, oviposition, and number of effective day-degrees which accumulated during the entire period of development. In computing the effective day-degrees for the stages, 50 degrees $F$. was taken as the zero of development and 88,85 , and 87 as the degree of maximum rate of development of the egg, larva, and pupa, respectively. By reason of the slightly different degrees of the maximum rate of development used for the different stages, the effective day-degrees of the different stages are units of not quite the same size and strictly speaking can not be added, but they differ so slightly that their sums, which are recorded in next to the last column in the tables, will serve as a basis for comparison.

In order that we might have a common unit in terms of which to express the accumulated effective day-degrees of the whole period from the deposition of the eggs to the emergence of the adult, it was necessary to use as the degree of the maximum rate of development a single point instead of 85,87 , and 88 , and 86 was selected as the nearest integral degree to the weighted average of the three. Using 50 as the zero of development and 86 as the degree of maximum rate of development, the effective day-degrees which accumulated during the combined egg, larval, and pupal periods were computed and recorded in the last column in the tables. All of the tables bearing on the life history of the insect (Tables 9-25) follow.

Erratum: P. 253, last line, for "follow" read "precede this page." 


\section{INCUBATION PERIOD}

Eggs of the first generation hatch during May and June. The period varied from 13 days during the cooler period early in May to 5 days during the warmer parts of June, the average being 9 days for the 243 eggs used in the life history series. In a larger series of observations consisting of 2664 eggs the period varied from 15 days to 6 days and the average was 9.3 days.

Eggs of the second generation hatched during July, August, and the early part of September and the incubation period varied from 5 to 9 days, depending upon the temperature, the average for 106 eggs from which transforming larvæ hatched being 6 days. The eggs of the transforming larvæ hatch prior to the middle of August, during the warmest part of the season. Their incubation period is shorter than the period of those from which the hibernating larvæ hatch later in the season. The average period for 1748 second-generation eggs was 6.6 days.

Eggs of the third generation hatched during the latter half of August and during September. The period varied from 6 to 15 days, the average for 302 eggs observed being 8.2 days.

Eggs of the hibernating generation, which consists of a very small per cent. of the first generation, a larger per cent. of the second, and all of the third generation, hatched during July, August, and September. The incubation period varied from 5 to 15 days, the average for the 242 eggs used in the life-history series being 6.7 days.

There was practically no difference between the incubation period of male and of female eggs; the average period of 340 of the former belonging to all generations was 7.9 days and of 395 of the latter 7.8 days.

Early in May and late in September the incubation period is comparatively long, varying from 12 to 15 days. It decreases in length till midsummer, when it is only 5 days, and then increases in length till the end of the season. The relation of temperature to the length of the incubation period is shown in Table 1 , page 235 .

\section{LARVAL PERIOD}

Transforming larvae.-The larval period of the first generation of larvæ reared in apples on the tree varied from a maximum of 42 days in the fruit and 9 days in the cocoon to a minimum of 18 days in the fruit and 1 day in the cocoon, the average period in the fruit being for males 27.6 days, for females 28.3 days, and for both males and females 27.9 days, and in the cocoon 4.2 days for both males and females.

The larval period of larvæ reared in picked apples averaged 21.2 days in the fruit and 4.4 days in the cocoon. The average total larval period of 227 first-generation larvæ reared in apples on the tree was 32.5 days and of 113 larvæ reared in picked fruit 25.8 days, the difference being 6.7 days. It was shown in Tables 3 and 4 that the accumula- 
tion of effective day-degrees was 86 greater during the period of larvæ reared in apples on the tree than during the period of larvæ reared in picked fruit. Since the average daily accumlation of day-degrees shown in these tables is approximately 22.75 , the difference in day-dgrees represents a difference of about 4 days in the period. That is, larvæ of the first generation may be expected to mature, on an average, about 4 days earlier in picked fruit than in fruit on the tree. The difference of 6.7 days shown above is, therefore, partly due to differences in temperature as well as to difference in the condition of the fruit in which the larvae fed.

The period of transforming larvæ of the second generation varied from a maximum of 33 days in the fruit and 7 days in the cocoon to a minimum of 17 days in the fruit and 1 day in the cocoon, the average period in the fruit being 22.6 days and in the cocoon 4.2 days. The average total period for 36 males was 26.3 and for 59 females 27.4 , the average for both being 26.8 days. The larval period of the female was about one day longer than that of the male.

Hibernating larvae.-In treating the larvæ of the hibernating generation no distinction has been made between larvæ of the different generations of the season which compose it. The period of the larvæ varied from a maximum of 46 days in the fruit and 269 days in the cocoon to a minimum of 16 days in the fruit and 209 days in the cocoon, the average period in the fruit being 29.0 days and in the cocoon 236 . The averagt total period of hibernating larvæ was 264 for the males, 269 for the females and 265 for both males and females.

The length of the larval period is affected by temperature and the character of food. It also varies in length in different individuals, even when they are under the same conditions as to temperature and food. The individual variations are well illustrated by the following examples. The periods of 16 larvæ which hatched on May 31, 1917, varied from 25 to 33 days and the periods of 16 others which hatched June 11, 1917, varied from 24 to 38 days, though the environment of the individuals of each lot was as nearly the same as it was possible to have it. It is not infrequent that in lots of individuals kept under the same conditions as to light, food, heat, and moisture the variation in the period from the average for the group equals 30 per cent. of the average period.

The relation of temperatures to the length of the larval period is shown in Tables 3 and 4, pp. 240, 243. A comparison of these two tables also shows that development is more rapid in picked apples than in apples on the tree. According to Table 3 , at an average mean daily temperature of $74.31^{\circ} \mathrm{F}$., the average period is 29.07 days in apples on the tree; and according to Table 4 , at an average mean daily temperature of $74.08^{\circ} \mathrm{F}$., the period is 25.69 days in picked fruit, or 3.4 days less than the period in apples on the tree. 


\section{PUPAL PERIOD}

Pupæ of the first generation were present from about the middle of June to the middle of August and the period varied from 8 to 14 days, the average being 10.6 days. Pupæ of the second generation were present during August and the first part of September. The period varied from 9 to 18 days, the average being 12.1 days. None of the third-generation larvæ pupated. The pupal period of this generation is therefore discussed in connection with the hibernating generation.

Pupæ of the hibernating generation were present during April, May, and the first part of June. The period varied from 39 days to 18 days, the average being 30.9 days. The average pupal period of the males and the females is practically the same. The period of the hibernating generation is much longer than that of the other generations because pupæ are present during early spring when the weather is cool.

\section{COMBINED EGG, LARVAL, AND PUPAL PERIODS}

The whole period of development is of the greatest importance since it is an index to the time which elapses from the deposition of the egg to the emergence of the adult. Since the deposition of eggs frequently begins within one day after the emergence of the female, the addition of one day to the period of development will give the time which elapses from the deposition of the egg of one generation to the deposition of the first egg of the following generation. For 103 males of the first generation the period varied from 40 to 59 days, the average being 51.1 days; for 131 females, the period varied from 43 to 62 days, the average being 52.4 days. For both males and females the average was 51.8 days.

The period of development for the individuals which hatch first in the spring is much longer than for those which hatch later, the minimum period for those hatching earliest being 50 days. We may expect, therefore, the deposition of the first eggs of the second generation to follow the deposition of the first eggs of the first generation in an average of about 51 days, though the average for this same period for the entire generation is only about 53 days. The above applies to individuals whose larval period was passed in apples on the tree. The period would be about 4 or 5 days shorter for those whose larvæ fed on picked apples.

The development period of 41 transforming males of the second generation varied from 38 to 50 days, the average being 43.7 days, and of 62 females from 33 to 56 days, the average being 45.4 days, the average for males and females being 44.7 days. The minimum period for the first individuals of the second generation was 37 days. We may, therefore, expect the deposition of eggs of the third generation to begin about 38 days after the deposition of the first eggs of the second generation.

The period from the first eggs of one generation to the first eggs of the next will vary with the season. The time which elapsed between 
the deposition of the first eggs of the first and second generations was 59 days in 1915, in 1916 it was 53 days, and in 1917, 43 days. The time which elapsed between the deposition of the first eggs of the second and the first of the third generation in 1915 was 46 days, and in 1916, 39 days.

The total development period for 97 males of the hibernating generation varied from 266 to 318 days, the average being 301 days, and for 103 females from 271 to 324 days, the average being 305 days, and the average for both males and females being 303 days.

\section{ADULT PERIOD}

As shown in Tables 13 and $14, * 60$ male adults of the first generation lived a maximum of 14 days, a minimum of 3 days, and an average of 6.4 days and 63 female adults a maximum of 15 days and an average of 7.4 days - an average of 6.9 days for both male and female adults. The averages given in Table 24 are for a smaller group and the averages are somewhat smaller.

Twenty-five male adults of the second generation lived a maximum of 12 days, a minimum of 3 days, and an average of 6.1 days; and 28 female adults lived a maximum of 24 days, a minimum of 5 days, and an average of 10.3 days (Tables 17,18 ). The averages for a different and somewhat larger group shown in Table 24 are 9.7 days for the males, 10.2 days for the females, and 10 days for both males and females.

Ninety-one males of the hibernating generation, according to Tables 21,22 , and 23 , lived a maximum of 24 days, a minimum of 3 days, and an average of 10.5 days, and 95 females lived a maximum of 27 days, a minimum of 5 days, and an average of 13.05 days. The averages of a smaller group, shown in Table 24 , are smaller, being 10 days for both males and females.

\section{TOTAL LIFE PERIOD}

The total life period of 24 males of the first generation, the larvæ of which were reared in apples on the tree, varied from 40 days to 68 days, the average being 57.4 days; and that of 29 females varied from 43 days to 68 days, the average being 59.8 , and the average for both males and females being 58.7 .

The total life period of 30 males of the first generation, the larvæ of which were reared in picked apples, varied from 42 to 60 days, the average being 51.6 days, and that of 44 females varied from 42 to 58 days, the average being 50.5 days, and the average for both males and females being 50.9 days.

Of the second generation the total life period of 25 males whose larvæ transformed, varied from 42 to 61 days, the average being 51.3 days, and that of 38 females varied from 45 to 65 days, the average being 53.6 days.

* See group of tables following p. 253. 
The remaining portions of the second generation and all of the third generation belong to the hibernating generation.

Of the hibernating generation, the total life period of 24 males varied from 286 days to 329 days, the average being 312 days, and that of 60 females varied from 288 to 330 days, the average being 314 days, and the average of both males and females being 313 days.

The maximum periods are for individuals whose larvæ left the fruit early in August, and the minimum periods for those whose larvæ left the fruit late in the fall. Larvæ which left the fruit late in the fall pupated and developed into moths at the same time as those which left the fruit earlier in the season as will be shown later on.

\section{OVIPOSITION}

Most of the first-generation females began to deposit eggs on the first or second day after emerging. The maximum number of eggs were laid on the second day. Oviposition ceased after the ninth day. The average number of eggs laid by each female was 39 in 1915 and 47.3 in 1916.

Oviposition by females of the second generation began later, but a majority of the moths had begun to oviposit by the third day. The maximum number of eggs in 1915 was laid on the sixth day and in 1916 on the second day after the emergence of the adults. This difference was no doubt due to the lower temperatures which prevailed in 1915. The average number of eggs laid by each female in 1915 was 66.3 and in 1916 it was 68.7 .

Oviposition by females of the hibernating generation takes place in the spring when the weather is cool. Very few females begin to oviposit on the first day after emerging, and a majority do not begin to oviposit till the fourth day. The maximum number of eggs was laid on the fourth day after the emergence of the female in 1915 and 1916 and on the fifth day in 1917. Oviposition practically ceased after the tenth day but a few eggs were laid as late as the sixteenth day. The average number of eggs laid by each female varied from 30.5 in 1916 to 42.4 in 1917. Low temperatures and heavy precipitation delay oviposition.

Adults when confined in small cages do not act normally. They attempt to escape from the cage and soon become disabled. Doubtless in the open the females live longer and deposit a larger number of eggs than the averages above given. The maximum number of eggs laid by a single female was 172 , the next highest numbers were $167,156,143$, 136 and 120.

\section{RELATION OF TEMPERATURE TO LENGTH OF DEVELOPMENT PERIOD}

The total effective day-degrees which accumulated during the three development periods were determined in two ways: first, by adding together the total effective day-degrees of the egg, larval, and pupal periods 
as determined by the use of 50 degrees $F$. as the zero of development and 88,85 , and 87 degrees $F$. as the degrees of the maximum rate of development of the egg, larva, and pupa respectively; and, second, by using 50 as the zero of development and 86 as the degree of the maximum rate of development for the entire period. The results are recorded in the next to the last and the last column, respectively, in the tables. The average total for the first generation, as shown in Table 24 , computed by the latter method, was 1122 , or 4 more than the total computed by the former method, and for the second generation it was 1119 , or 15 more computed by the latter method than by the former method. The maximum difference amounts to less than half a day in the development period and hence the latter method may be used for practical purposes, and the following discussion will have reference to results obtained by it.

The average total day-degrees for the female is about 20 greater than for the male and 10 greater than the average for the male and the female, which indicates that the female has a slightly longer period than the male.

There is a large variation in individuals as to the length of the development period and hence in the total effective day-degrees which accumulate during the period. In individual cases these totals varied from 814 to 1475 , but less than 4 per cent. of them were below 950, and less than 2 per cent above 1350 . Since some of the extremely high and extremely low totals recorded were probably due to errors in observations, 950 and 1350 may be regarded as the approximate extremes, 1120 being the average.

The total day-degrees which accumulated during the development period of individuals whose larvæ were reared in picked fruit, as shown in Table 25, were 1010, or 109 less than the average for individuals whose larvæ were reared in apples on the tree, showing that larvæ develop more rapidly in picked fruit than in fruit on the tree.

\section{Seasonal History of the Codling-moth, 1915*}

The seasonal history of the codling-moth was determined at Olney by large-cage experiments, supplemented by data secured from band collections and the life history studies.

\section{LARGE-CAGE SERIES}

The cages (Fig. 5, 6, 7) used in these experiments were large enough to cover entire trees and were used for the purpose of keeping the generations under observation separate, so that the number of generations, the time of beginning, and the time of ending could be definitely determined. The events especially noted were dates when the first and the last moths

* A preliminary report on the observations and experiments of the year 1915 was published in the 29th report of the State Entomologist of Illinois, issued in January, 1916, and also in the annual report of the State Horticultural Society for 1915. That paper ("On the Life History of the Codling-moth," by Stephen A. Forbes and Pressley A. Glenn) contains some data and discussions not repeated in the present article. 
were liberated in their respective cages, dates when the first and the last larvæ left the fruit, when they pupated, and when the moths emerged.

First generation, 1915.-The first moths which emerged from hibernating larvæ were liberated in cage No. 1, as follows:

\begin{tabular}{|c|c|c|c|}
\hline \multicolumn{2}{|c|}{ Date } & Males & Females \\
\hline May & 1 & 4 & 1 \\
\hline & 2 & 2 & 0 \\
\hline " & 3 & 8 & 4 \\
\hline “ & 4 & 0 & 1 \\
\hline " & 5 & 4 & 2 \\
\hline " & 6 & 12 & 6 \\
\hline
\end{tabular}

The first eggs were found in the cage on the morning of May 6 . They had probably been laid during the evening of the preceding day. These eggs hatched May 17. There was a heavy June drop of apples all over the orchard and the drop was especially heavy from some of the trees caged. In cage No. 1, 275 apples were picked up May 20 and placed in battery jars. They averaged only about one-half inch in diameter. June 4, 800 were picked up, June 9, 700, June 12, 300, and June 19, 150. At this time only a few apples remained on the tree. Larvæ began to leave the fruit in this cage June 22 .

In another cage; in which moths had been liberated on May 12 , or 12 days later than in cage No. 1, larvæ began to leave the fruit June 22. Judging from this, had normal conditions prevailed in cage No. 1, larvæ should have begun to leave the fruit 11 days earlier than they did-that is, on June 11. This date corresponds very well with the date when the first larvæ were taken under bands, which was June 12 .

The last moths of the hibernating generation were liberated in cage No. 2, as follows:

\begin{tabular}{|c|c|c|c|}
\hline \multicolumn{2}{|c|}{ Date } & Males & Females \\
\hline $\mathrm{MaJ}$ & 31 & 11 & 9 \\
\hline June & 1 & 4 & 5 \\
\hline & 2 & 3 & 4 \\
\hline " & 3 & 5 & 8 \\
\hline " & 8 & 6 & 12 \\
\hline “ & 9 & 2 & 3 \\
\hline " & 10 & 1 & 1 \\
\hline “ & 11 & 1 & 3 \\
\hline “" & 12 & 2 & 3 \\
\hline “ & 13 & 1 & 4 \\
\hline & 15 & & \\
\hline
\end{tabular}

Larvæ began to leave the fruit in cage No. 2 on July 6 and continued to leave the fruit till August 1. Pupation continued till August 4, and moths continued to emerge till August 17. Two larvæ which left the fruit July 17 and 19 respectively, hibernated. All other larvæ kept under observation transformed or died. 
Second generation, 1915.- The first moths from cage No. 1 were liberated in cage No. 3 , to rear the first of the second generation, as follows :

\begin{tabular}{cccc}
\multicolumn{2}{c}{ Date } & Males & Females \\
July & 1 & 2 & 3 \\
" & 3 & 1 & 1 \\
“ & 5 & 11 & 11 \\
. & 6 & 5 & 10
\end{tabular}

The first eggs were laid July 3 , the first larvæ hatched July 11, the first larvæ left the fruit August 2 (these larvæ were lost), the first pupæ were found August 7, and the first moths emerged August 17.

The first larvæ to hibernate, left the fruit August 9. All larvæ which left the fruit after August 15, hibernated. The last pupa was obtained August 19 and the last moth September 2.

The last adults of the first generation to emerge from materials reared in cage No. 2 were liberated in cage No. 4, to rear the last of the second generation, as follows:

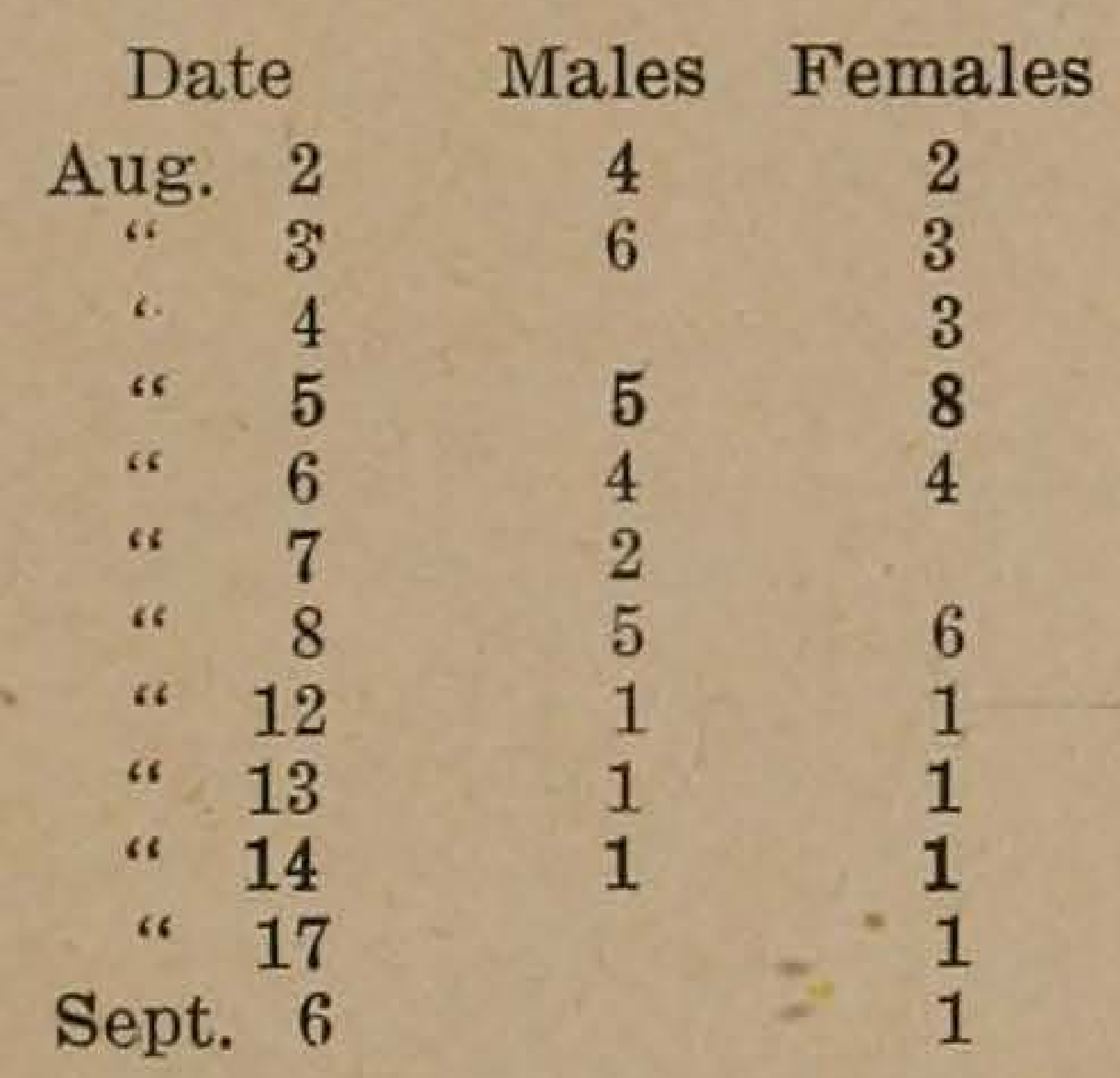

The last eggs were laid September 12. The last of these eggs hatched September 17. Larvæ began to leave the fruit in this cage September 12 and continued to leave the fruit till November 1, the date of the last observation. All of the larvæ reared in this cage hibernated.

Third generation, 1915.- To rear the third generation, moths which were reared in cage No. 3 were liberated in cage No. 5, as follows:

$\begin{array}{ccc}\text { Date } & \text { Males } & \text { Females } \\ \text { Aug. } 22 & 2 & 2 \\ \text { ". } 23 & 4 & 4 \\ \text { “ } 24 & 7 & 4 \\ \text { “ } 25 & 2 & 1 \\ \text { ". } 27 & 2 & 1 \\ \text { “ } 29 & 1 & 1 \\ \text { Sept. } 3 & & 2 \\ \text { " } 5 & & 1\end{array}$

The first eggs were observed August 26 and the first larvæ left the fruit September 28. The last egg observed was laid September 1 and hatched September 9. Since adults of the second generation continued 
to emerge till September 5, oviposition no doubt continued till about September 15 and hatching till about September 25. Larvæ continued to leave the fruit till November 1, the date of the last observation. All the larvæ reared in cage No. 5 hibernated.

\section{BAND COLLECTIONS}

Hibernating generation, 1914-1915.-Hibernating larvæ were collected at Centralia and Olney April 12, 13, and 14. About 1400 were kept at Olney and the remaining 600 were kept under observation at Ozark. From 5 to 10 per cent. of the larvæ collected on the above dates had pupated. Judging from the time the first moths emerged, pupation began about April 9. Pupation was very irregular owing to variable weather conditions. It was at its maximum at Olney from April 22 to April 29, and continued till June 2. Larvæ kept under observation at Urbana, Illinois, 100 miles north of Olney, began to pupate April 20 and continued to pupate till May 24.

The first moths emerged at Olney April 27, the maximum emergence occurred May 14 and 15, and moths continued to emerge till June 26. A record of pupation and emergence of adults at Olney will be found in Table 26.

Collections made in 1915.- The bands used at Olney were two-ply burlap bands about 4 inches wide. They were placed on the trees about June 1 and examined daily until the first larvæ were found. After that they were examined every third day. When collected the number of males and females were recorded, and, at Olney, they were preserved for the purpose of observing pupation and emergence (Table 26).

Larvæ of the first generation began to leave the fruit June 12. The maximum number were leaving the fruit about June 25 . The larvæ of the first and second generations overlapped during the last week in July and the first week in August. Development was greatly retarded during August on account of unusually cool weather, and was greatly accelerated during the second and third weeks of September on account of unusually warm weather. These weather influences are indicated in the band records by the relatively small number of larvæ collected during August and the large number during September. The falling off in band collections after September 21 was due largely to the fact that apple-picking began at about that date.

A daily record of the band collections, life-history observations, and climatic conditions at Olney in 1915 have been brought together in Table 26 for ready reference. 
TABLE 28-Seasonal Histoby of the Codling-MOTH at OLNEY in 1915

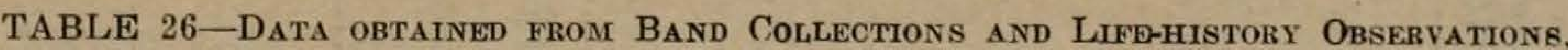
with accompanying Climatic Data, Olney, 1915

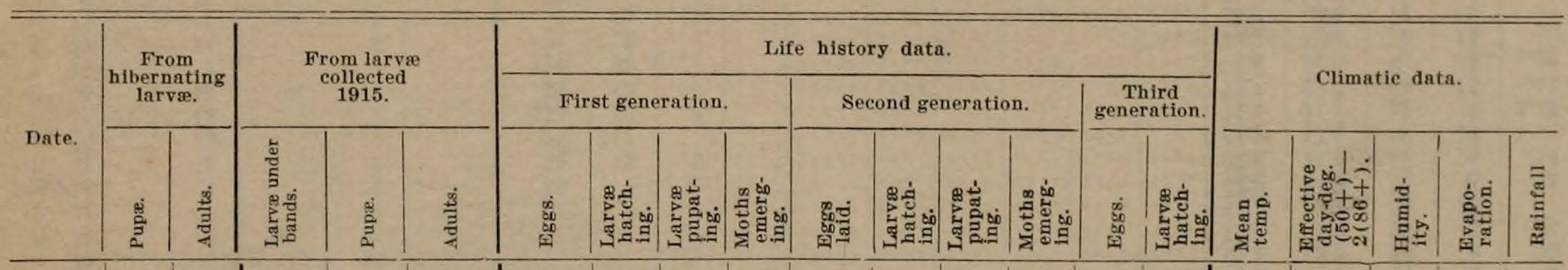

\begin{tabular}{|c|c|c|c|}
\hline Apr. & 1 & & \\
\hline " & 2 & $\ldots$ & $\ldots$ \\
\hline & 3 & $\ldots$ & $\ldots$ \\
\hline " & 4 & $\ldots$ & $\ldots$ \\
\hline " & 5 & $\ldots$ & $\ldots \ldots$ \\
\hline “ & 6 & $\ldots$ & $\ldots \ldots$ \\
\hline " & 7 & $\ldots$ & $\ldots$ \\
\hline “ & 8 & $\ldots$ & $\ldots$ \\
\hline$"$ & 9 & $\ldots \ldots$ & $\ldots$ \\
\hline “ & 10 & $\ldots$ & $\ldots$ \\
\hline “ & 11 & $\cdots$ & $\cdots$ \\
\hline “" & 12 & $\cdots \cdots$ & $\cdots$ \\
\hline$"$ & 13 & 1 & $\cdots$ \\
\hline " & 14 & $\cdots$ & $\cdots$ \\
\hline " & 15 & 1 & $\cdots$ \\
\hline "، & 16 & 3 & $\cdots$ \\
\hline " & $\begin{array}{l}17 \\
18\end{array}$ & $\begin{array}{l}4 \\
1\end{array}$ & $\cdots$ \\
\hline " & 19 & 6 & $\ldots$ \\
\hline$"$ & 20 & 8 & ... \\
\hline$"$ & 21 & 9 & $\ldots$ \\
\hline$"$ & 22 & 10 & $\ldots$ \\
\hline " & 23 & 13 & $\ldots$ \\
\hline$"$ & 24 & 13 & $\ldots$ \\
\hline " & 25 & 8 & $\ldots$ \\
\hline$"$ & 26 & 22 & $\ldots$ \\
\hline$"$ & 27 & 9 & 1 \\
\hline " & 28 & 6 & 2 \\
\hline$"$ & $\begin{array}{c}99 \\
29\end{array}$ & 14 & \\
\hline
\end{tabular}
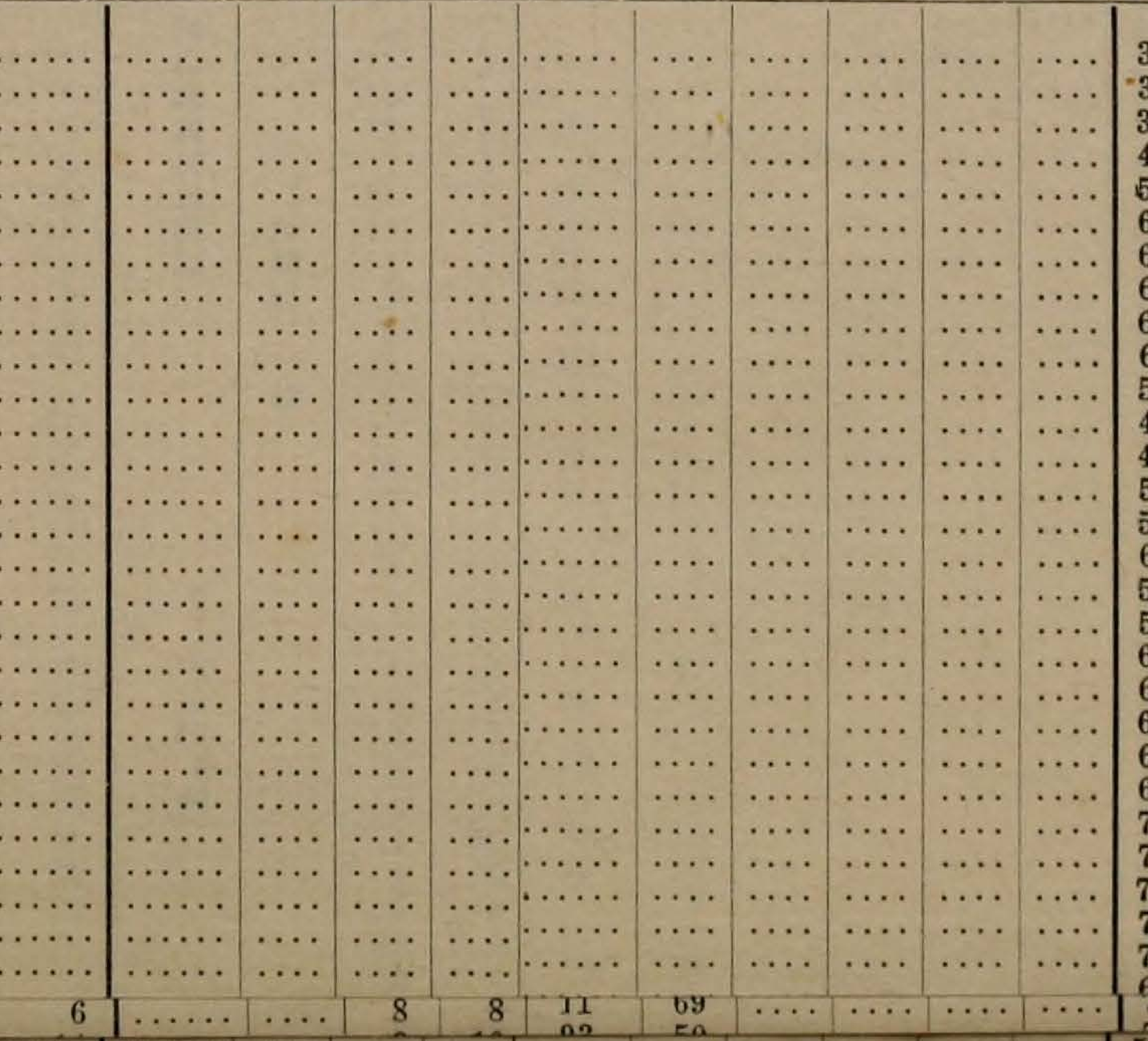

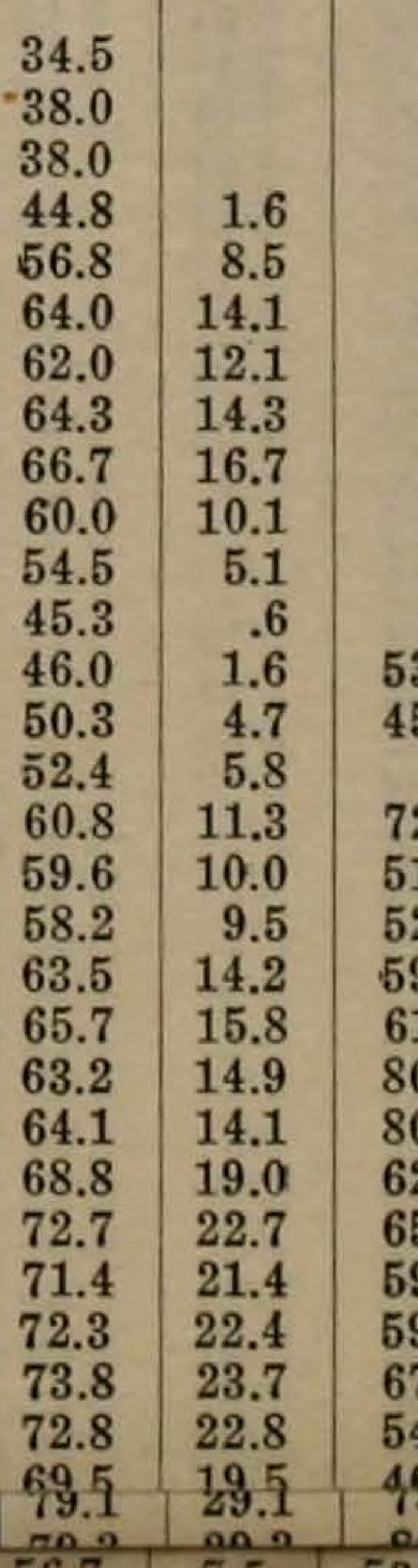




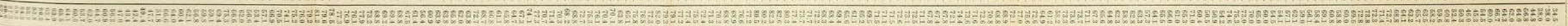

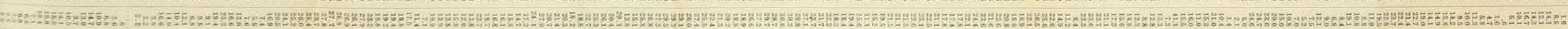

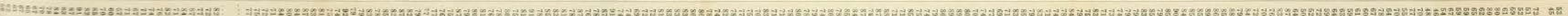

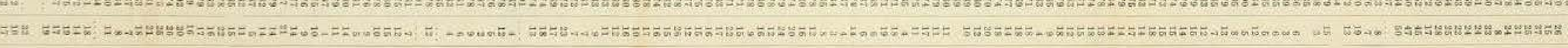


TABLE 27-NUMBER OF LARVAE COLLECTED ON VABIOUS DATES IN 1915. NUMBFR TRANSFORMING AND NUMBER HIBERNATTNG.

\begin{tabular}{|c|c|c|c|c|c|c|c|c|c|c|c|c|}
\hline \multirow{2}{*}{\multicolumn{2}{|c|}{$\begin{array}{l}\text { Date of } \\
\text { leaving } \\
\text { applee. }\end{array}$}} & \multicolumn{4}{|c|}{$\begin{array}{c}\text { Number of } \\
\text { larve collected. }\end{array}$} & \multirow{2}{*}{ 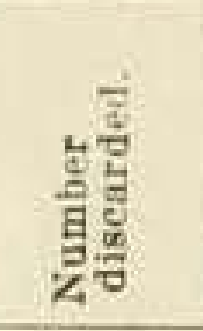 } & \multicolumn{2}{|c|}{$\begin{array}{l}\text { Number } \\
\text { transforming, }\end{array}$} & \multicolumn{2}{|c|}{$\begin{array}{l}\text { Per cent, } \\
\text { tranaforming }\end{array}$} & \multicolumn{2}{|c|}{$\begin{array}{l}\text { Number } \\
\text { of advitis } \\
\text { emerglug. }\end{array}$} \\
\hline & & $\begin{array}{l}\text { Total } \\
\text { number. }\end{array}$ & $\begin{array}{l}\text { Average } \\
\text { number } \\
\text { per day. }\end{array}$ & कू & $\begin{array}{l}\text { Number } \\
\text { of each } \\
\text { Bex. }\end{array}$ & & 1915. & 1916. & 1915. & 1916. & 1915. & 1918. \\
\hline \multicolumn{2}{|c|}{ June $11-16$} & 18 & 5 & $\begin{array}{l}\text { M. } \\
\text { F. }\end{array}$ & $\begin{array}{r}10 \\
8\end{array}$ & $\begin{array}{l}2 \\
2\end{array}$ & $\begin{array}{l}8 \\
6\end{array}$ & & $\begin{array}{l}100 . \\
100 .\end{array}$ & & $\begin{array}{l}7 \\
4\end{array}$ & \\
\hline " & $17 \cdot 19$ & 48 & 16 & $\mathrm{M}$. & 26 & 1 & 25 & .......... & 100. & & 21 & \\
\hline .. & & & 10 & F & 22 & 7 & 15 & & 100. & & 14 & \\
\hline & $20-22$ & 96 & 32 & $\begin{array}{l}\text { M. } \\
\text { F. }\end{array}$ & $\begin{array}{l}50 \\
46\end{array}$ & $\begin{array}{r}6 \\
16\end{array}$ & $\begin{array}{l}44 \\
30\end{array}$ & $\ldots \ldots \ldots$ & $\begin{array}{l}100 . \\
100 .\end{array}$ & & $\begin{array}{l}41 \\
24\end{array}$ & \\
\hline . & $23-25$ & 211 & 70 & M. & 77 & 14 & 63 & .......... & 100. & …..... & $\begin{array}{l}24 \\
63\end{array}$ & \\
\hline & & & & F. & 134 & 34 & 100 & $\ldots \ldots$ & 100. & & 99 & \\
\hline ". & $26-28$ & 189 & 63 & $\begin{array}{l}\mathrm{M} \text {. } \\
\mathrm{F}\end{array}$ & $\begin{array}{r}86 \\
103\end{array}$ & $\begin{array}{l}26 \\
35\end{array}$ & $\begin{array}{l}60 \\
68\end{array}$ & … & 100. & $\ldots \ldots \ldots$ & 50 & \\
\hline . & 29-July 1 & 113 & 38 & M. & 46 & $\begin{array}{r}50 \\
6\end{array}$ & $\begin{array}{l}68 \\
40\end{array}$ & & $\begin{array}{l}100 . \\
100 .\end{array}$ & $\ldots \ldots \ldots$ & $\begin{array}{l}66 \\
40\end{array}$ & \\
\hline & & & 38 & F. & 67 & 15 & 52 & & 100. & & 52 & \\
\hline July & y. 2.3 & 101 & 51 & M. & 48 & 8 & 40 & $\ldots \ldots \ldots$ & 100. & ..... & 40 & \\
\hline .. & 4. 7 & 133 & & M. & $\begin{array}{l}56 \\
57\end{array}$ & $\begin{array}{l}6 \\
5\end{array}$ & 49 & 3 & $\begin{array}{r}100 . \\
94.2\end{array}$ & 5.8 & $\begin{array}{l}47 \\
49\end{array}$ & 3 \\
\hline & & & 33 & $\mathrm{~F}$. & 76 & 15 & 59 & 2 & 95.1 & 3.3 & 59 & 2 \\
\hline " & $8-10$ & 66 & 22 & M. & 29 & 15 & 14 & & 100. & ....... & 14 & \\
\hline & $11-13$ & 37 & & M. & $\begin{array}{l}37 \\
19\end{array}$ & $\begin{array}{l}8 \\
7\end{array}$ & 29 & ........ & $\begin{array}{l}100 . \\
100\end{array}$ & & 26 & \\
\hline & & & 12 & F. & 18 & 6 & 12 & …........ & 100. & …..... & 12 & \\
\hline$"$ & $14-16$ & 46 & 15 & M. & 23 & 3 & 20 & $\ldots \ldots \ldots$ & 100. & & 20 & \\
\hline. & & & & F. & 23 & 6 & 17 & ......... & 100. & & 17 & \\
\hline " & $17-19$ & 39 & 13 & $\begin{array}{l}\text { M. } \\
\text { F. }\end{array}$ & $\begin{array}{l}18 \\
21\end{array}$ & $\begin{array}{r}3 \\
11\end{array}$ & $\begin{array}{l}15 \\
10\end{array}$ & $\ldots \ldots \ldots$ & $\begin{array}{l}100 . \\
100 .\end{array}$ & $\ldots \ldots \ldots$ & $\begin{array}{l}12 \\
10\end{array}$ & \\
\hline .. & $20-22$ & 40 & 13 & M. & 20 & 4 & 16 & $\ldots \ldots \ldots$ & 100. & $\ldots \ldots$ & 16 & \\
\hline & & & & F. & 20 & 1 & 19 & ......... & 100. & ......... & 19 & \\
\hline " & $23-24$ & 31 & 16 & M. & 14 & $\begin{array}{l}0 \\
4\end{array}$ & $\begin{array}{l}14 \\
13\end{array}$ & $\ldots \ldots$ & 100. & $\ldots \ldots \ldots$ & 14 & \\
\hline a & $25-28$ & 40 & 10 & M. & 19 & 6 & 13 & $\ldots \ldots$ & $\begin{array}{l}100 . \\
100 .\end{array}$ & $\ldots \ldots \ldots$ & $\begin{array}{l}13 \\
13\end{array}$ & \\
\hline & & & 10 & F. & 21 & 6 & 14 & 1 & 93.3 & 6.7 & 14 & 1 \\
\hline “ & $29-31$ & 19 & 6 & $\begin{array}{l}\mathrm{M} \text {. } \\
\mathrm{F} \text {. }\end{array}$ & 9 & 4 & 5 & $\ldots \ldots \ldots$ & 100. & ........ & 3 & \\
\hline Ang. & $1-3$ & 21 & 7 & $\begin{array}{l}\text { F. } \\
\text { M. }\end{array}$ & $\begin{array}{r}10 \\
6\end{array}$ & $\frac{1}{0}$ & $\begin{array}{l}9 \\
6\end{array}$ & $\ldots \ldots \ldots$ & $\begin{array}{l}100 . \\
100\end{array}$ & $\ldots \ldots \ldots$ & 8 & \\
\hline 40 & & & & F. & 15 & 6 & 9 & & 100. & …...... & 4 & \\
\hline "* & 4. 6 & 65 & 22 & $\begin{array}{l}\text { M. } \\
\text { F }\end{array}$ & $\begin{array}{l}32 \\
32\end{array}$ & 4 & 26 & 2 & 92.9 & 7.1 & 22 & 2 \\
\hline. & 7- 9 & 100 & 33 & M. & $\begin{array}{l}33 \\
46\end{array}$ & $\begin{array}{r}17 \\
8\end{array}$ & $\begin{array}{l}15 \\
34\end{array}$ & 1 & $\begin{array}{l}93.8 \\
89.5\end{array}$ & 6.2 & 11 & 1 \\
\hline & & & & F. & 54 & $\begin{array}{l}8 \\
9\end{array}$ & 45 & 4 & $\begin{array}{l}89.5 \\
100 .\end{array}$ & 10.5 & $\begin{array}{l}31 \\
45\end{array}$ & 4 \\
\hline ." & $10-12$ & 82 & 27 & M. & 40 & 12 & 17 & 11 & 60.7 & 39.3 & 17 & 11 \\
\hline & & & & F. & 42 & 16 & 20 & 6 & 77.1 & 22.9 & 20 & 6 \\
\hline ." & $13-14$ & 54 & 27 & M. & $\begin{array}{l}26 \\
28\end{array}$ & 7 & $\begin{array}{l}13 \\
13\end{array}$ & 6 & 68.4 & 31.6 & 13 & 6 \\
\hline .. & $15-18$ & 145 & 26. & M. & 78 & $\begin{array}{l}12 \\
34\end{array}$ & 7 & $\ddot{3}$ & $\begin{array}{l}81.3 \\
17.1\end{array}$ & 18.7 & 13 & 3 \\
\hline 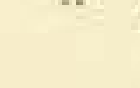 & & 145 & 36 & F. & 70 & $\begin{array}{l}34 \\
36\end{array}$ & 10 & $\begin{array}{l}34 \\
24\end{array}$ & 29.4 & $\begin{array}{l}81.9 \\
70.6\end{array}$ & 6 & $\begin{array}{l}31 \\
20\end{array}$ \\
\hline " & $19-21$ & 136 & 45 & M. & 74 & 18 & 3 & 53 & 5.4 & $\begin{array}{l}70.6 \\
94.6\end{array}$ & $\begin{array}{l}4 \\
3\end{array}$ & $\begin{array}{l}20 \\
53\end{array}$ \\
\hline & & & & F. & 62 & 19 & 6i: & 37 & 14.0 & $\begin{array}{l}94.6 \\
86.0\end{array}$ & 6 & 37 \\
\hline ". & $22-24$ & 174 & 58 & M. & 89 & 40 & 1 & 48 & 2.0 & 98.0 & 1 & 48 \\
\hline.. & & & & $F$. & 85 & 39 & $\frac{2}{2}$ & 44 & 4.4 & 95.6 & 2 & 44 \\
\hline " & $25-27$ & 161 & 54 & $\begin{array}{l}\text { M. } \\
\text { F. }\end{array}$ & $\begin{array}{l}75 \\
86\end{array}$ & $\begin{array}{r}8 \\
18\end{array}$ & 0 & $\begin{array}{l}65 \\
68\end{array}$ & 3. & 97. & 2 & 51 \\
\hline$"$ & $28-30$ & 139 & & $\mathrm{M}$. & $\begin{array}{l}86 \\
71\end{array}$ & $\begin{array}{l}18 \\
25\end{array}$ & 0 & $\begin{array}{l}68 \\
46\end{array}$ & $, \ldots, \ldots$ & 100. & 0 & 45 \\
\hline & & & 46 & F. & $\begin{array}{l}71 \\
68\end{array}$ & $\begin{array}{l}25 \\
23\end{array}$ & 0 & $\begin{array}{l}46 \\
45\end{array}$ & , $\ldots \ldots$ & 100. & 0 & 22 \\
\hline$"$ & 31-Sept. 2 & 143 & 48 & M. & 69 & 18 & 0 & $\begin{array}{l}45 \\
51\end{array}$ & $\ldots, \ldots$ & 100. & $\begin{array}{l}0 \\
0\end{array}$ & $\begin{array}{l}30 \\
20\end{array}$ \\
\hline & & & & F. & 74 & 10 & 0 & 64 & $\ldots \ldots+\ldots$ & $\begin{array}{l}100 . \\
100 .\end{array}$ & 0 & 28 \\
\hline Sept. & - $3-4$ & 176 & 88 & M. & 83 & 18 & 0 & 65 & $\ldots \ldots \ldots$ & 100. & 0 & 34 \\
\hline$\because$ & & & & $F$ & 93 & 34 & 0 & 59 & $\cdots$ & 100. & 0 & 22 \\
\hline$"$ & $5-8$ & 434 & 109 & M. & 193 & 46 & 1 & 146 & .7 & 99.3 & 1 & 124 \\
\hline " & $9-11$ & 369 & & F. & 241 & 89 & $\frac{2}{0}$ & 150 & 1.3 & 98.7 & 2 & 127 \\
\hline & & & 123 & $\begin{array}{l}\text { M. } \\
\mathrm{F}\end{array}$ & $\begin{array}{l}148 \\
221\end{array}$ & $\begin{array}{l}31 \\
66\end{array}$ & $\begin{array}{l}0 \\
0\end{array}$ & 117 & $\ldots \ldots \ldots$ & 100. & 0 & 89 \\
\hline ." & $12-14$ & 393 & 121 & M. & 166 & $\begin{array}{l}66 \\
44\end{array}$ & 0 & 155 & $\ldots \ldots+\cdots$ & 100. & 0 & 107 \\
\hline & & & & F. & 227 & 59 & 0 & $\begin{array}{l}122 \\
168\end{array}$ & $\ldots \ldots \ldots$ & $\begin{array}{l}100 . \\
100\end{array}$ & $\begin{array}{l}0 \\
0\end{array}$ & \\
\hline " & $15-17$ & 395 & 132 & M. & 176 & 53 & 0 & 123 & tents & 100. & 0 & $\begin{array}{r}139 \\
62\end{array}$ \\
\hline & & & & E. & 219 & 88 & 0 & 131 & $, \ldots, \ldots, \ldots$ & 100. & 0 & \\
\hline " & 18.20 & 232 & 77 & M. & 103 & 34 & 0 & 69 & , n..., & 100. & 0 & 57 \\
\hline & & & & F. & 129 & 47 & 0 & 82 & n...n & 100. & 0 & 57 \\
\hline " & $21-23$ & 118 & 39 & M. & 61 & 7 & 0 & 54 & $\ldots \ldots \ldots$, & 100. & 0 & 40 \\
\hline * & 24.25 & 111 & & F. & 57 & 6 & 0 & 51 & .......... & 100. & 0 & 37 \\
\hline & $-7=28$ & & 37 & M. & $\begin{array}{l}52 \\
59\end{array}$ & $\begin{array}{l}3 \\
9\end{array}$ & $\begin{array}{l}0 \\
0\end{array}$ & $\begin{array}{l}49 \\
50\end{array}$ & $\ldots \ldots \ldots \ldots$ & 100. & 0 & 43 \\
\hline * & $26-29$ & 205 & 51 & $\mathrm{M}$ & 103 & $\begin{array}{r}9 \\
29\end{array}$ & $\theta$ & $\begin{array}{l}50 \\
74\end{array}$ & $\ldots \ldots \ldots$ & 100. & 0 & 40 \\
\hline & & & & F. & 102 & 17 & 0 & $\begin{array}{l}74 \\
85\end{array}$ & $\ldots \ldots \ldots \ldots$ & $\begin{array}{l}100 . \\
100\end{array}$ & $\begin{array}{l}0 \\
0\end{array}$ & $\begin{array}{l}48 \\
35\end{array}$ \\
\hline 18 & $30.0 \mathrm{ct} .5$ & 169 & 28 & M. & 89 & 17 & 0 & 72 & $\ldots \ldots \ldots$ & $\begin{array}{l}100 . \\
100 .\end{array}$ & 0 & 60 \\
\hline & & & & F. & 80 & 5 & $\theta$ & 75 & …, & 100. & 0 & 64 \\
\hline Oet. & $6-11$ & 67 & 11 & M. & 37 & 3 & $\theta$ & 34 & .......... & 100. & 0 & 29 \\
\hline$*$ & & & & E. & 30 & 1 & 0 & 29 & & 100. & 0 & 25 \\
\hline 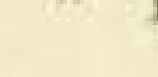 & $12-18$ & 128 & 18 & $\mathrm{M}$. & $\begin{array}{l}54 \\
74\end{array}$ & 16 & 0 & 38 & $\ldots \ldots \ldots$ & 100. & 0 & 32 \\
\hline 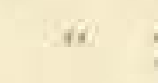 & $19-25$ & 79 & & M. & 33 & $\begin{array}{r}12 \\
9\end{array}$ & $\begin{array}{l}0 \\
0\end{array}$ & $\begin{array}{l}62 \\
24\end{array}$ & (......... & 100 & $0_{0}^{0}$ & $\begin{array}{l}53 \\
24\end{array}$ \\
\hline & & & 11 & $F$ & 46 & 14 & 0 & 32 & ……. & $\begin{array}{l}100 . \\
100\end{array}$ & 0 & $\begin{array}{l}24 \\
32\end{array}$ \\
\hline$"$ & 26-Nov, 1 & 48 & 7 & M. & 28 & 2 & 0 & 26 & ............ & 100. & 0 & 22 \\
\hline & & & & F: & 20 & ....... & 0 & 20 & .......... & 100. & 0 & 15 \\
\hline & Totain & 5371 & & $\underset{M}{\frac{M}{N}}$ & $\begin{array}{l}2480 \\
2891 \\
5371\end{array}$ & $\begin{array}{r}596 \\
826 \\
1421\end{array}$ & $\begin{array}{r}548 \\
692 \\
1170\end{array}$ & $\begin{array}{l}1336 \\
1444 \\
2780\end{array}$ & $\begin{array}{l}29.09 \\
30.04 \\
39.54\end{array}$ & $\begin{array}{l}70.91 \\
69.96 \\
70.46\end{array}$ & $\begin{array}{r}515 \\
589 \\
1104\end{array}$ & $\begin{array}{l}1009 \\
1031 \\
2040\end{array}$ \\
\hline
\end{tabular}


TABLE 28-Seasonal History of the Codling-moth at OLNey in 1915

\begin{tabular}{|c|c|c|c|c|c|c|}
\hline Generation & Period & Earliest & $\begin{array}{l}\text { Relatively } \\
\text { numerous }\end{array}$ & Maximum & $\begin{array}{l}\text { Relatively } \\
\text { numerous }\end{array}$ & Latest \\
\hline \multirow{4}{*}{ Hibernating } & Pupæ & Apr. 9 & Apr. 19 & Apr. 26 & May & June 2 \\
\hline & Adults & " 27 & May 10 & May 15 & “ 22 & " 24 \\
\hline & Eggs & May 5 & " 16 & & " 31 & “ $30^{*}$ \\
\hline & Young larvæ & “ 17 & “ 26 & & June 12 & July $9 \div$ \\
\hline \multirow[t]{5}{*}{ First } & Mature larvæ & June 12 & June 19 & June 25 & July 7 & Aug. 9 \\
\hline & Pupæ & " 17 & “ 25 & “ 29 & “ 10 & “ 17 \\
\hline & Adults & “ 28 & July 10 & July 12 & 22 & Sept. 6 \\
\hline & Eggs & July 3 & " 13 & & “ 28 & " 12 \\
\hline & Young larvæ & “ 11 & “ 13 & & Aug. 3 & " 17 \\
\hline \multirow[t]{4}{*}{ Second } & Mature larvæ & Aug. 2 & Aug. 18 & Sept. 17 & 20 & -7 \\
\hline & Pupæ & “ & & Aug. 10 & & Aug. $18 \S$ \\
\hline & Adults & “ 16 & & & & Sept. $5 \S$ \\
\hline & Eggs & " 22 & & & & " 13 \\
\hline \multirow[t]{2}{*}{ Third } & Young larvæ & " 29 & & & & “ 19 \\
\hline & Mature larvæ & Sept. 22 & & & & $-\ddagger$ \\
\hline
\end{tabular}

- The last eggs observed were laid June 26.

† The latest hatching-date record is June 27, the egg having been laid June 18 .

‡ Larvæ were collected Nov. 1, the date of the last observation. These dates are from materials collected from cages, but larvæ from the much larger band-collections continued to pupate till
A ug. 30, and moths to emerge till Sept. 16. No doubt egg-laying continued till about Sept. 26 and hatching of larva till about Oct. 7 . 
Data based on the band collections give in a general way the distribution as to relative numbers of individuals of each stage throughout the season; the life history data indicate only approximately the general periods during which the different stages of each generation occurred. Since only a small number of individuals were used in the life-history observations as compared with the number of individuals in the whole orchard, activities in the orchard, no doubt, began somewhat earlier and continued somewhat later than the life history data indicate. This is especially true in regard to the date of the deposition of the last eggs, the hatching of the last larvæ, etc., of each generation, because usually the last individuals were very few in number and were mostly females, many of which died without oviposition or laid infertile eggs.

Hibernation, 1915.-The first larvæ to hibernate left the fruit between July 3 and July 7 . There was a period of three cool days, including July 4, 5, and 6 , when the mean daily temperatures were $65.2,61.1$ and 65.5 degrees F. respectively. For the remainder of July the temperature was much higher. The cool days no doubt account for the early hibernation of some of the larvæ which left the fruit from July 3 to July 7. Hibernation proper began with larvæ which left the fruit between August 3 and August 6 , after which the per cent. that hibernated increased rapidly until August 20. Practically all larvæ maturing after this date hibernated. The weather during the second week in September was unusually warm and it was expected that larvæ would again begin to transform, but only three out of about 1000 which were under observation responded to this increased temperature.

Of the total number of larvæ collected, 46.2 per cent. were males and 53.8 per cent. were females; 29.1 per cent. of the males and 30.1 per cent. of the females collected, transformed; and 70.9 per cent. of the males and 69.9 per cent. of the females hibernated.

SUMMARY OF THE SEASONAL HISTORY, 1915

From the foregoing data the seasonal history of the codling-moth at Olney in 1915 may be constructed quite accurately (Table 28, preceding this page). 
In Chart 1, these same details of the seasonal history are shown. An attempt is made here to show in graphic form the relative numbers of pupæ, adults, eggs, young larvæ, and mature larvæ that appeared from day to day, all of which have been averaged for three-day periods. The chart is divided vertically into columns for the months from April to October and longitudinally by lines representing elevations above certain base lines, the degree of elevation being represented by the figures in the column to the left of the chart. The average numbers of pupæ, adults, eggs, young larvæ and mature larvæ which appeared daily during each of the three-day periods are represented by the black vertical columns. At the top of the chart the three broods of pupæ are represented, next comes the three broods of moths, then the eggs, then young larvæ, and lastly, the larvæ leaving the fruit. The three broods are indicated very distinctly in the parts of the chart representing different stages. In the lower part of the chart the mean daily temperatures above $50^{\circ}$ are represented, also averaged for three-day periods.

\section{RECORDS FOR OTHER POINTS THAN OLNEY}

Thus far the discussion has been confined almost entirely to the Olney records. A few words additional will suffice for the less complete records at Ozark, Plainview, and Princeton. The codling-moth was so scarce at the last-mentioned place as to render the records of little value. A distinct third generation was detected at Ozark the same as at Olney. Since only band records were kept at Plainview no evidence of the third generation was noticed there, though it doubtless occurred and would have been detected if a daily, or even a weekly, record of the emergence of moths from the larvæ collected under bands had been kept.

The first moths observed at Ozark emerged April 28, or one day later than at Olney. The first larvæ of the first generation were taken under bands at Ozark June 2, at Olney June 12, and at Plainview June 25. The first of the second-brood moths emerged at Ozark June 20, or 8 days before the first moths of this brood emerged at Olney, and the first of the second-brood larvæ hatched at Ozark July 2, or 9 days before they hatched at Olney.

\section{GENERAL REMARKS ON THE SEASON}

March and April, 1915, were dry months and much warmer than normal, causing the pupation of the hibernating larvæ to begin early, but during May, June, July, and August the temperature averaged from 4 to 7.5 degrees cooler than normal, and the rainfall for these months was much above normal. These months were characterized by many cool, cloudy, rainy days, all of which tended to retard the development of the codling-moth, and to reduce the numbers of each generation that survived. This threw the beginning of the pupation of the first third-brood pupæ as late as August 7, which was only two weeks before pupation ended for the season. For this reason the third generation was very small. 


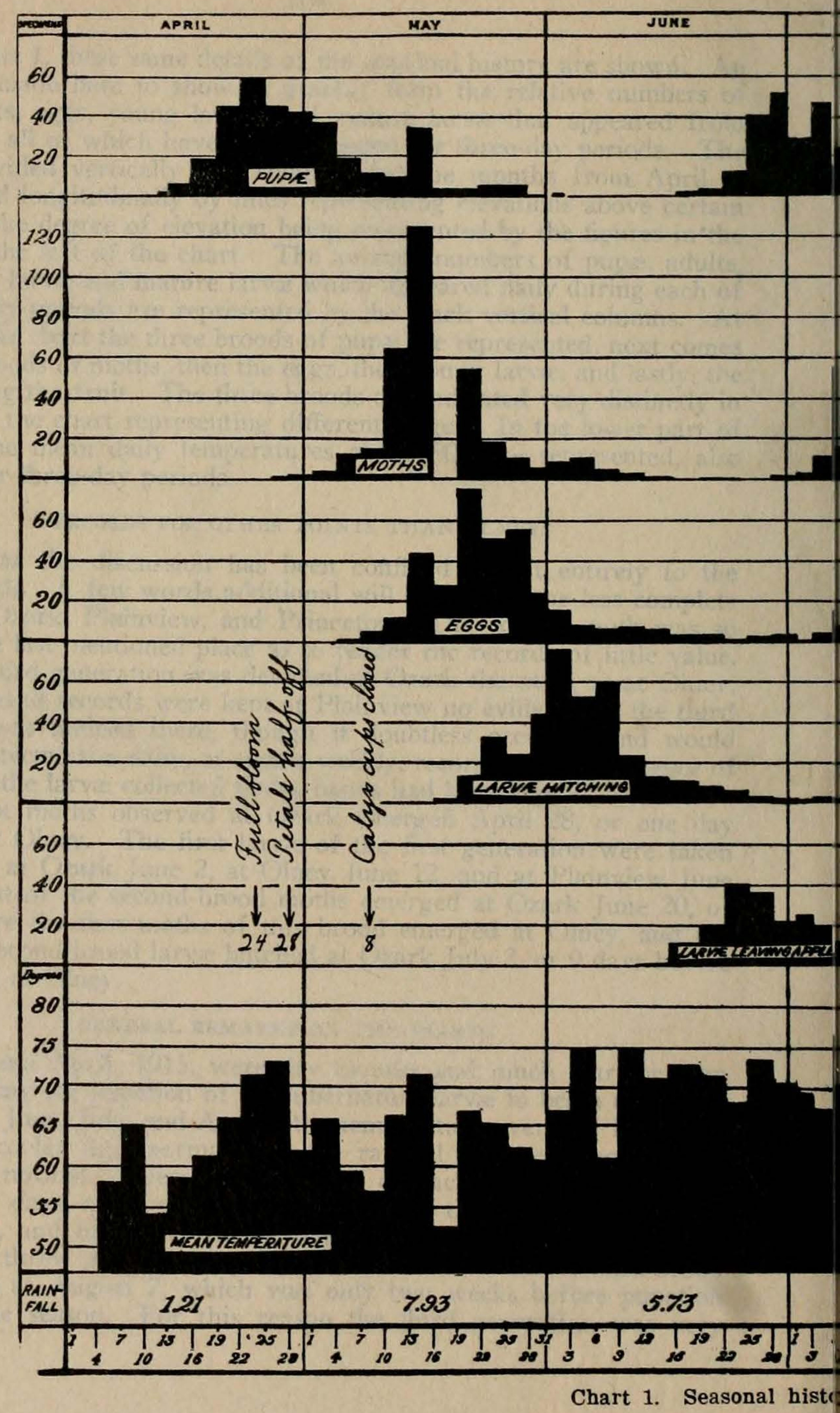




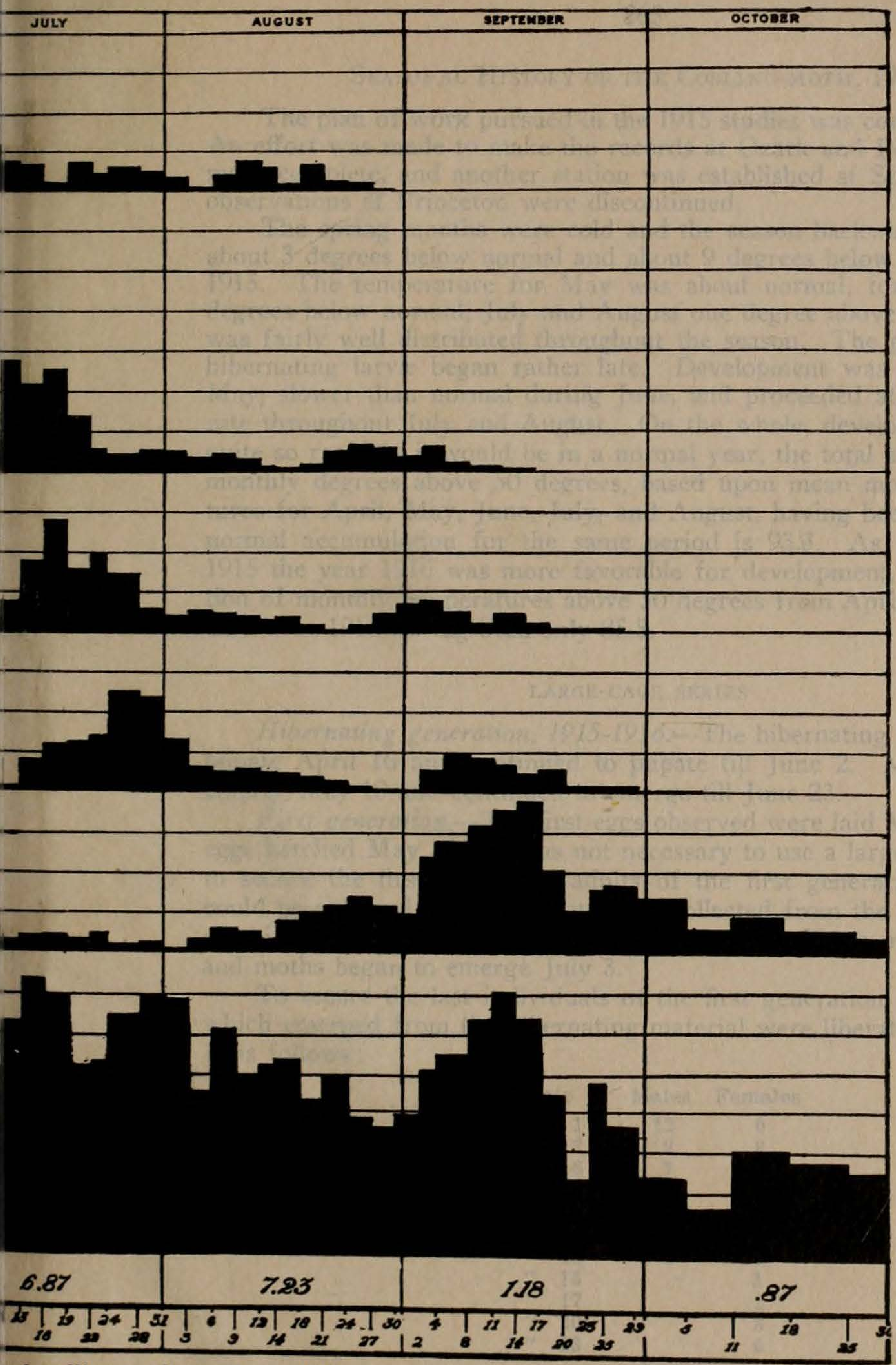

of codling-moth at Olney, 1915. 
Seasonal History of the Codling-moth, 1916

The plan of work pursued in the 1915 studies was continued in 1916. An effort was made to make the records at Ozark and Plainview a little more complete, and another station was established at Springfield. The observations at Princeton were discontinued.

The spring months were cold and the season backward. April was about 3 degrees below normal and about 9 degrees below what it was in 1915. The temperature for May was about normal, for June about 5 degrees below normal, July and August one degree above normal. Rain was fairly well distributed throughout the season. The pupation of the hibernating larvæ began rather late. Development was normal during May, slower than normal during June, and proceeded at the maximum rate throughout July and August. On the whole, development was not quite so rapid as it would be in a normal year, the total accumulation of monthly degrees above 50 degrees, based upon mean monthly temperatures for April, May, June, July, and August, having been 89 while the normal accumulation for the same period is 93.3. As compared with 1915 the year 1916 was more favorable for development, the accumulation of monthly temperatures above 50 degrees from April to August inclusive in 1915 having been only 85.5 .

\section{LARGE-CAGE SERIES}

Hibernating generation, 1915-1916.-The hibernating larvæ began to pupate April 16 and continued to pupate till June 2. Moths began to emerge May 10 and continued to emerge till June 23.

First generation.-The first eggs observed were laid May 14. These eggs hatched May 25. It was not necessary to use a large cage in order to secure the first pupæ and adults of the first generation, since they could be obtained from the first larvæ collected from the bands. Larvæ of this generation began to leave the fruit June 20, to pupate June 24 , and moths began to emerge July 3 .

To secure the last individuals of the first generation, the last adults which emerged from the hibernating material were liberated in cage No. 1 , as follows:

\begin{tabular}{|c|c|c|c|}
\hline \multicolumn{2}{|c|}{ Date } & Males & Females \\
\hline June & 1 & 12 & 0 \\
\hline & 3 & 9 & 8 \\
\hline " & 6 & 1 & 3 \\
\hline " & 8 & 6 & 3 \\
\hline " & 10 & 5 & 4 \\
\hline " & 12 & 11 & 11 \\
\hline " & 13 & 1 & 3 \\
\hline “ & 15 & & 1 \\
\hline " & 17 & & 2 \\
\hline " & 20 & & 3 \\
\hline “ & 23 & & 6 \\
\hline
\end{tabular}


Larvæ began to mature and leave the fruit July 11 and continued to leave the fruit till August 9. The last pupation occurred August 14, and the last emergence on August 20. All the larvæ of this generation transformed.

Second generation.-For the purpose of getting the earliest individuals of the second generation, the first moths of the first generation were liberated in large-cage No. 2, as follows:

\begin{tabular}{|c|c|c|c|}
\hline \multicolumn{2}{|c|}{ Date } & Males & Females \\
\hline July & 6 & 3 & 3 \\
\hline & 7 & 1 & 1 \\
\hline “ & 8 & 1 & 3 \\
\hline " & 9 & 2 & 1 \\
\hline " & 10 & 3 & 2 \\
\hline " & 12 & 5 & 7 \\
\hline " & 14 & 13 & 8 \\
\hline " & 16 & 2 & 12 \\
\hline
\end{tabular}

July 20, many apples in the cage were showing signs of worminess. Larvæ began to leave the fruit August 3, to pupate August 7, and moths began to emerge August 16.

To rear the last individuals of the second generation, moths which emerged from cage No. 1 on or after August 10 were liberated in cage No. 3 as follows:

\begin{tabular}{|c|c|c|}
\hline Date & Males & Females \\
\hline Aug. 16 & 4 & 4 \\
\hline " 11 & 8 & 3 \\
\hline 12 & 4 & 3 \\
\hline $1:$ & 1 & 1 \\
\hline 15 & 1 & 1 \\
\hline 17 & 1 & \\
\hline 18 & 2 & \\
\hline 20 & & 1 \\
\hline
\end{tabular}

Larvæ began to leave the fruit September 20 and continued to leave it until observations closed, October 12. All the larvæ reared in this cage hibernated.

Third generation.-To rear the third generation, moths which emerged from cage No. 2 were liberated in cage No. 4 as follows:

\begin{tabular}{|c|c|c|}
\hline Date & Males & Females \\
\hline Aug. 16 & 1 & 1 \\
\hline “ 18 & 2 & \\
\hline " 20 & 1 & 1 \\
\hline “ 23 & 5 & 1 \\
\hline " 24 & 1 & 3 \\
\hline “ 25 & & 4 \\
\hline “ 26 & & 1 \\
\hline “ 27 & & 2 \\
\hline " 30 & 1 & 1 \\
\hline Sept. 2 & 1 & 2 \\
\hline “ 8 & 2 & 1 \\
\hline
\end{tabular}

The adults liberated September 8 were from the second generation of the life-history series. 


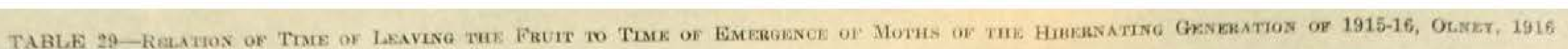

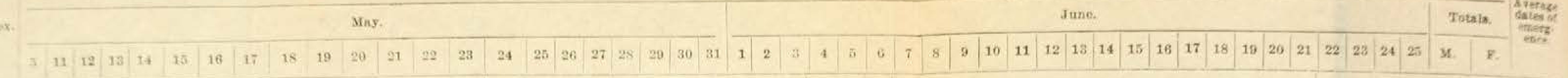

1915

Juty 3. ? M. ? 
The first eggs were observed August 19. These hatched August 23. The first mature larvæ left the fruit between September 26 and October 3 , and larva continued to leave the fruit till October 23--the date of the last observation.

Thus the presence of a partial third brood was again demonstrated this year at Olney. Larvæ of the first generation were entering the fruit from May 25 to about July 4, and larvæ of the second generation from about July 13 to September 1, and larvæ of the third generation from August 23 to about September 15.

\section{BAND COLLECTIONS}

Hibernating generation, 1915-1916.--Pupation of the hibernating larvæ collected from bands in 1915 began at Ozark April 10, at Olney, Plainview, and Springfield April 13, and closed at Ozark May 31, at Olney June 17, and at Springfield May 26.

Moths began to emerge at Ozark and at Olney May 11 and at Springfield May 10. They continued to emerge at Ozark till June 19, at Olney till June 30 (Table 29, foot-note), and at Springfield till June 28. Pupation was very irregular on account of changes in the weather conditions.

The dates when the larvæ left the fruit had no appreciable influence on the time of pupation and emergence the following spring. The average date of the emergence of males was May 21, and of the females May 23. We should naturally expect that hibernating larvæ maturing in July would develop into moths earlier in the spring than those maturing later in the season, but this is not the case. The male moths in three collections emerged an average of from one to five days later thais the females; in two collections the average dates of emergence of both sexes were the same; and in the other eighteen collections the males emerged an average of from one to seven days earlier than the females. For the whole series the average date of emergence of the males was 2.6 days earlier than that of the females.

Collections made in 1916.-Band collections were made at Ozark, Olney, and Springfield. The results are shown in Table 30.

The first eggs were observed August 19. These hatched August 23.

While the first moths of the hibernating generation observed at the three places emerged at about the same time, the first larvæ of the first generation matured 10 days later at Olney than at Ozark and 9 days later at Springfield than at Olney. The first pupæ were observed at Olney June 24, and at Springfield July 2; the first adult at Olney emerged July 3, and at Springfield July 11 (Table 31 ). The second and third broods of pupæ and moths are very clearly indicated in these pupation and emergence records. The pupation of the second brood at Olney began June 24 and continued till the first or second week in August, at which time the beginning of the pupation of the third brood is indicated by the increase in the number of pupx. The second brood of moths began to emerge July 3 and continued to emerge until about the second 
week in August. The beginning of the emergence of the third brood is indicated by the increase in the number of moths emerging daily after the 16th of August. Judging from the above data the third brood was about equal in number to the second brood.

TABLE 30-Record of Larvae collected under Bands at Ozark, Olney, and SPRINGFIELD IN 1916

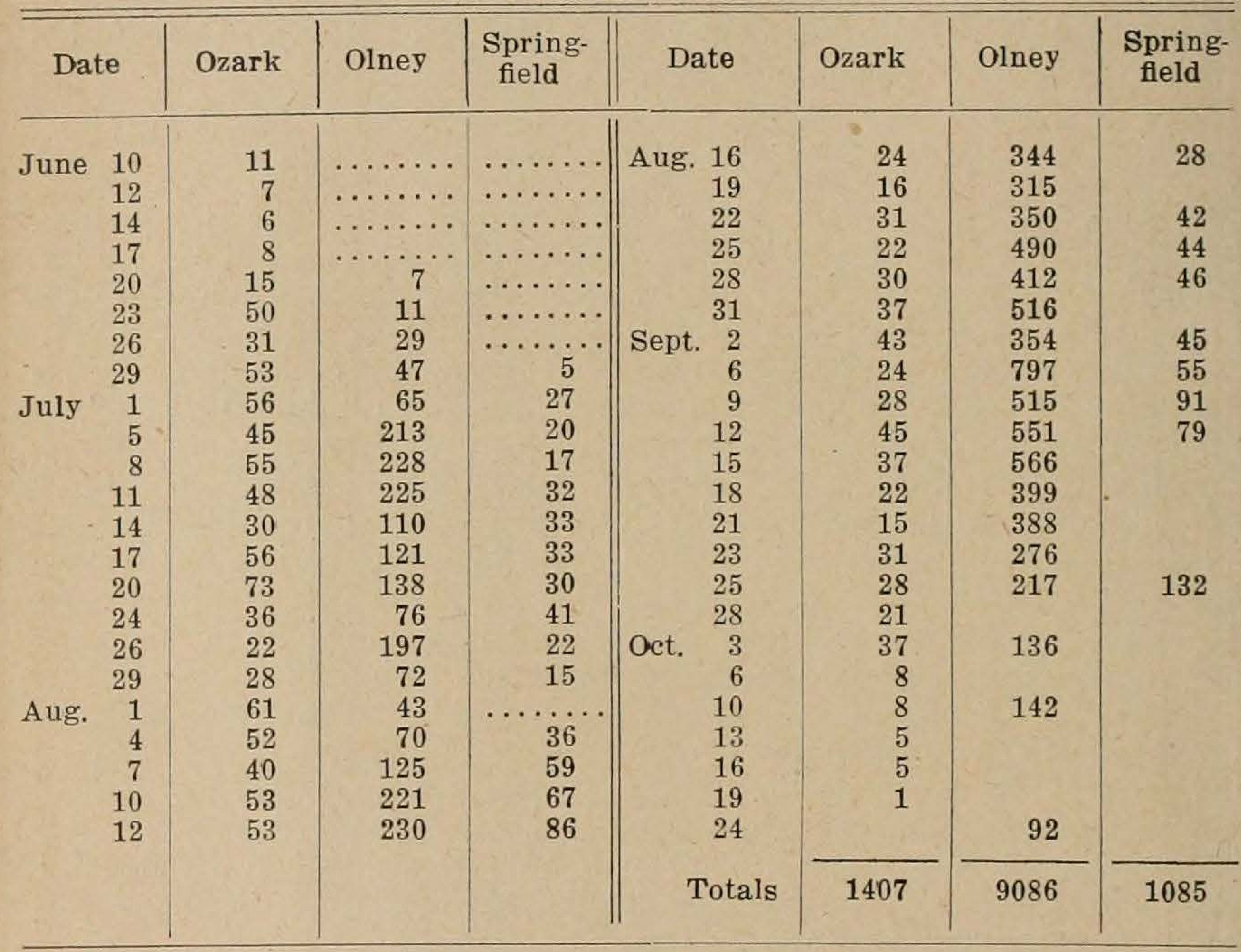

Hibernation, 1916.-Table 32 shows the dates between which the transition was made from transforming to hibernating larvæ.

Hibernation began with larvæ which left the fruit between August 4 and August 7, but hibernation in considerable numbers began with larvæ collected between August 16 and August 19. Practically all larvæ collected after August 28 hibernated. This was fully a week later than the date on which active hibernation began in 1915. It will be seen, then, that a large per cent. of the larvæ which left the fruit during the first three weeks in August transformed; it will also be seen by referring to Table 30 that larvæ of the second generation were leaving the fruit in large numbers during this period. This accounts for the relatively large size of the third generation in 1916.

Of the 9088 larvæ collected at Olney, 4100 , or about 45 per cent., were males, and 4988 , or about 55 per cent., were females. 


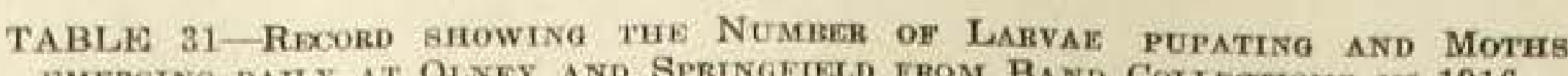

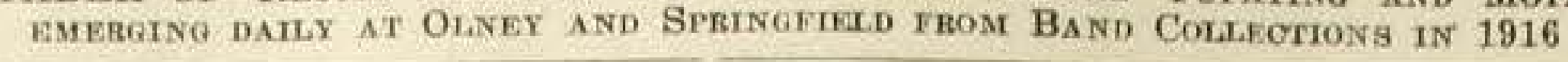

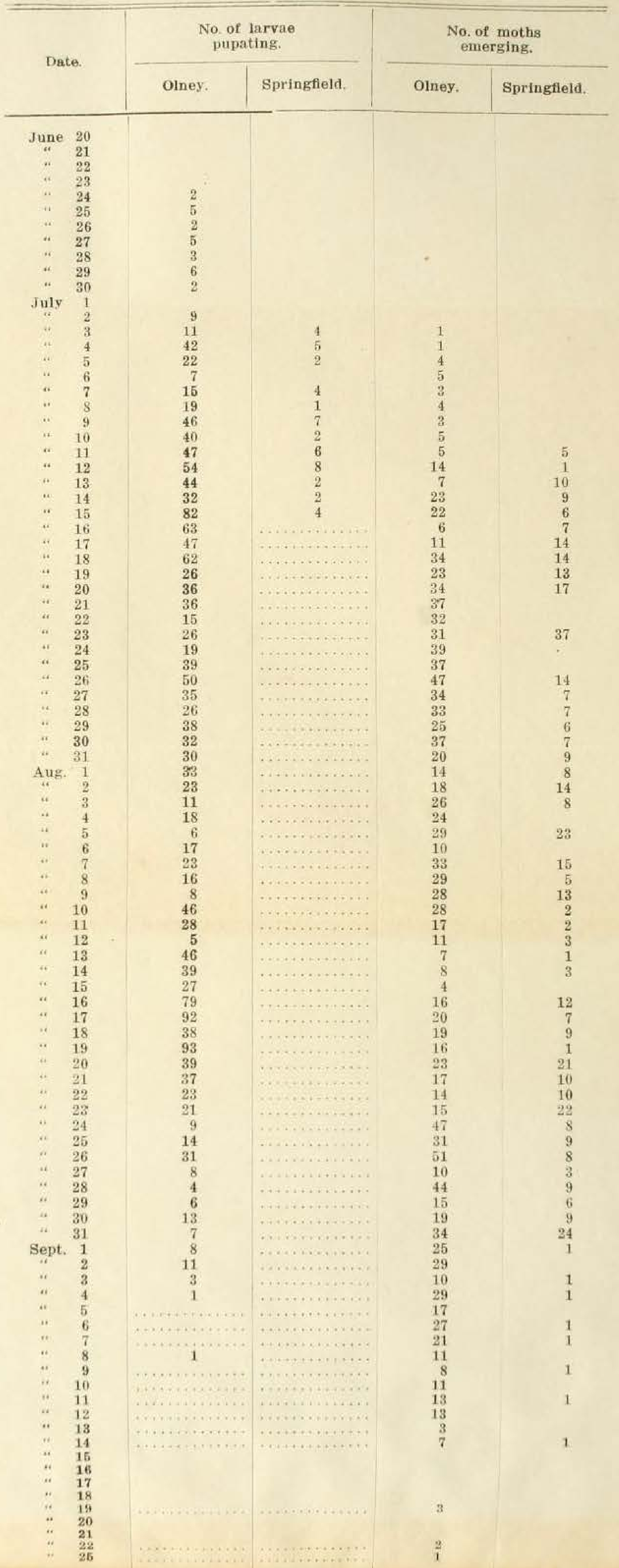


TABLE 32-NuMBER OF LARVAE COLlected on VARIous DATES in 1916. NUMBER TRANAFORMTING AND NTMBER HUERNATING

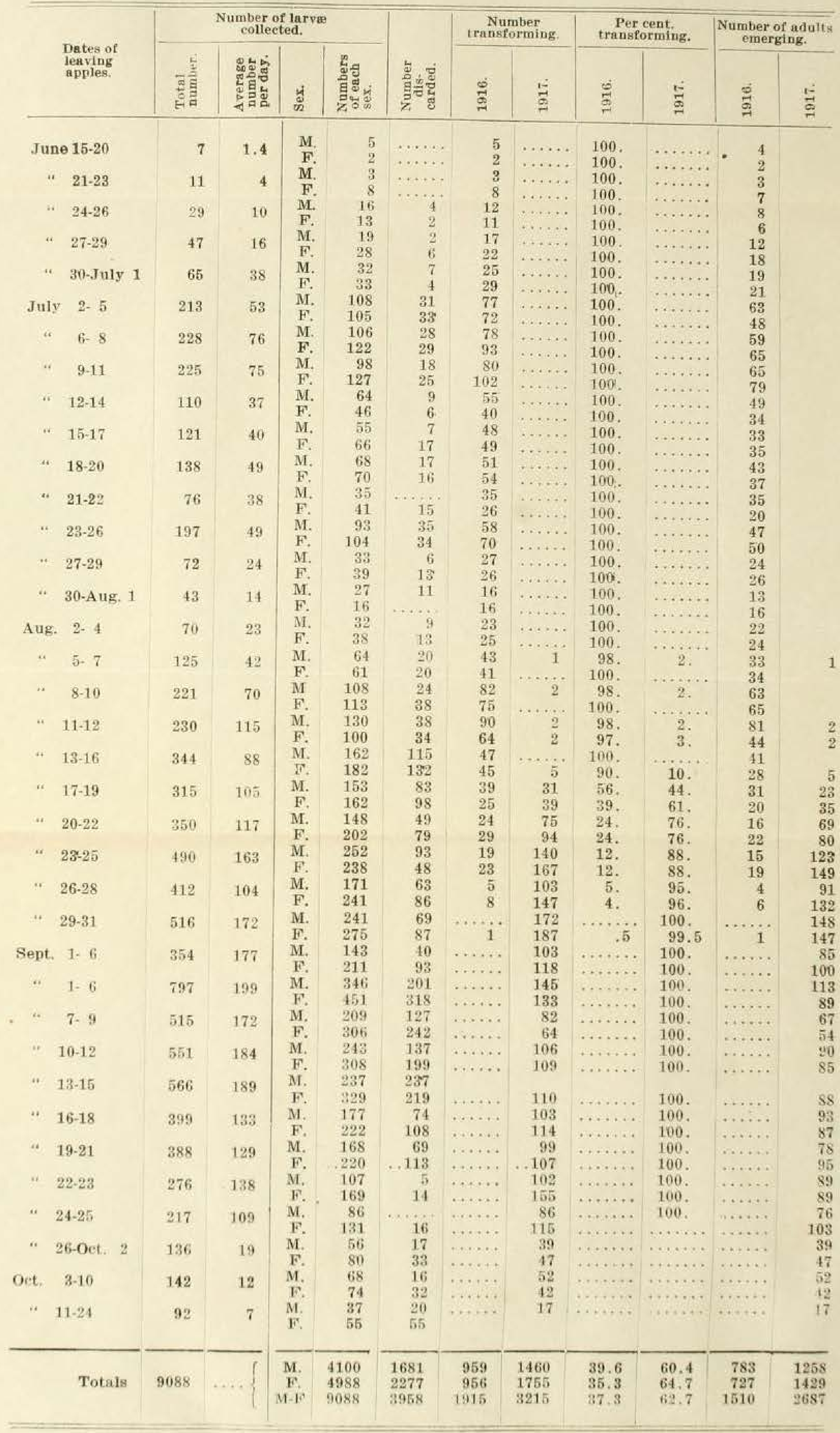




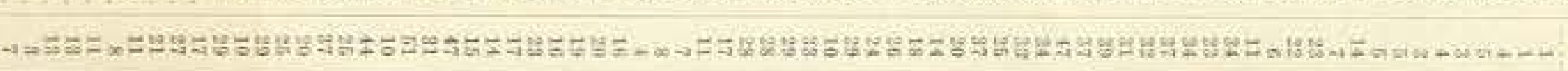

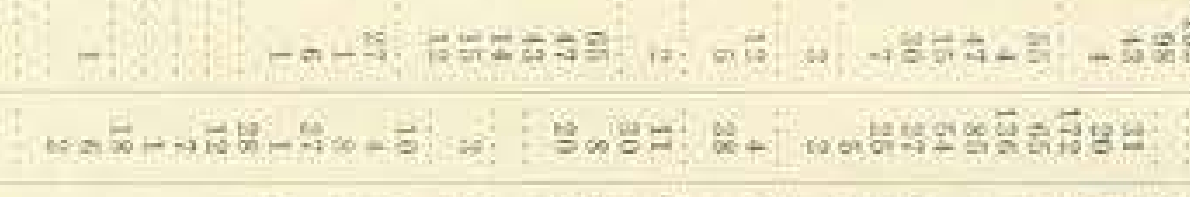

โ⿻上丨

\begin{tabular}{|c|c|c|}
\hline 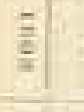 & : $5: 55$ & 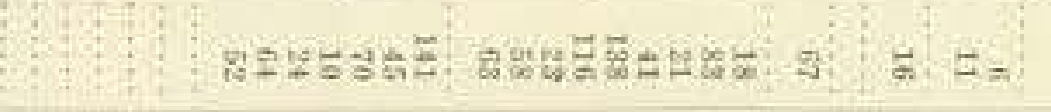 \\
\hline 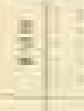 & Af: & 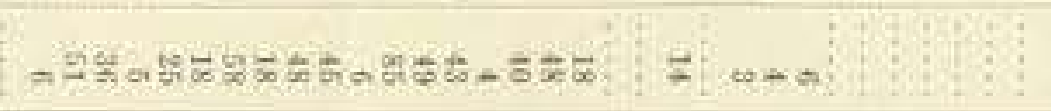 \\
\hline 立 & 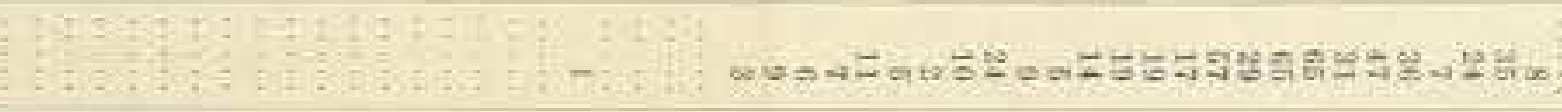 & \\
\hline 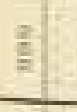 & 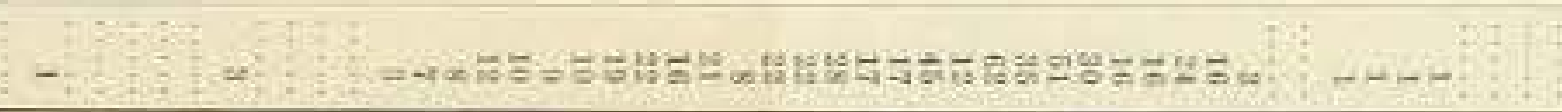 & \\
\hline
\end{tabular}

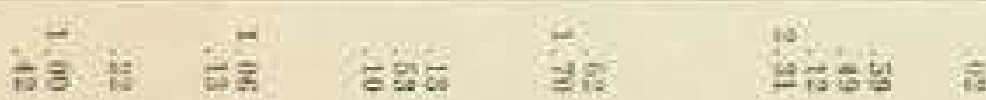

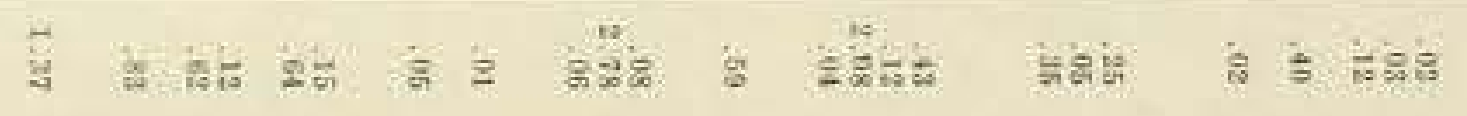

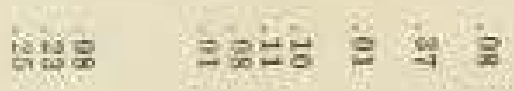


Of the males, 39.6 per cent., and of the females, 35.3 per cent. transformed, 60.4 per cent. of the males and 64.7 per cent. of the females hibernated. Altogether, 37.3 per cent. of those collected transformed and 62.7 per cent. hibernated. Undoubtedly a much larger per cent. hibernated than the above figures indicate, since the collections do not include all the hibernating larvæ that left the fruit late in the season, after collections had ceased. It is probably true that three fourths or more of the larvæ which develop during a given season hibernate. This being the case it is very evident that if a very large per cent. of them did not perish during the winter, the first generation in the spring would be much larger than it usually is.

A daily record of the band collections, life-history observations, and climatic conditions at Olney in 1916 have been brought together in Table 33 for ready reference.

\section{SUMMARY OF THE SEASONAL HISTORY, 1916}

From the foregoing data and an occasional reference to original notes we can summarize quite accurately the seasonal history of the codling-moth for 1916 (Table 34).

Chart 2 gives a graphic representation of the seasonal history of the codling-moth in 1916, the daily temperatures above 50 degrees F., and the monthly rainfall.

The number of pupations observed daily are shown in the top section of the chart, the number of moths emerging daily in the second section from the top, the number of eggs deposited in the third, the number of larvæ hatched in the fourth, and the number of larvæ leaving the fruit in the fifth. The daily temperatures above 50 degrees $F$. are shown in the bottom section, beneath which is given the rainfall for each month.

The order of events in the seasonal history is indicated by the numbers from 1 to 12 in the chart.

Thus: 1 indicates the first brood of pupæ

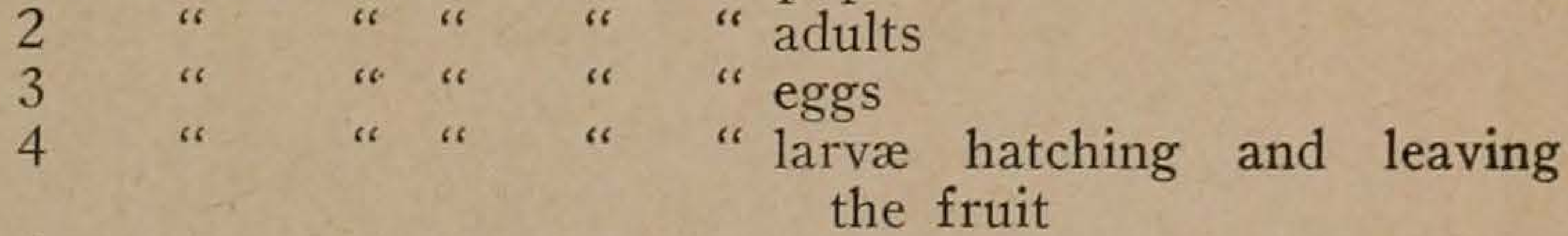

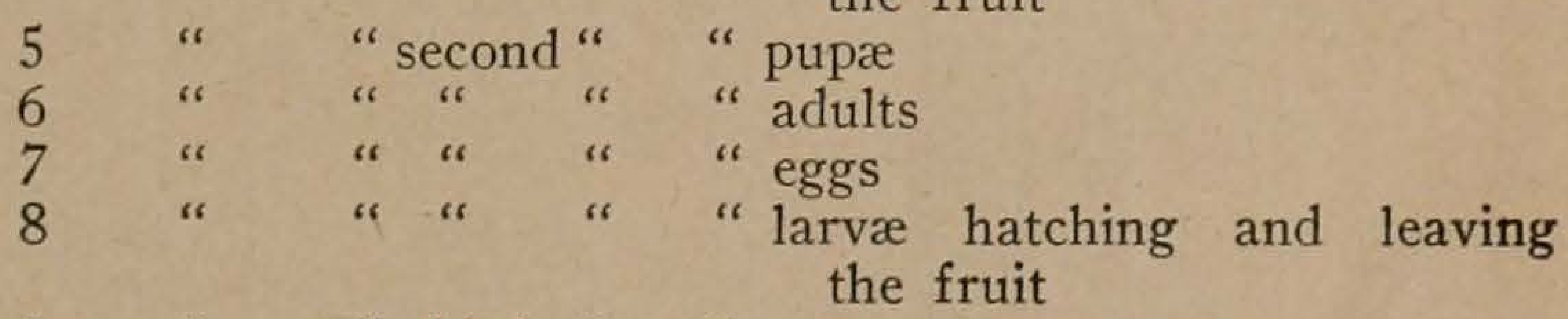

$$
\begin{aligned}
& \begin{array}{r}
9 . \\
10
\end{array} \\
& 11 \text { " " " " " " " " " " " " " " } \\
& 12 \text { " " " " "larvæ hatching and leaving } \\
& \text { the fruit. }
\end{aligned}
$$


TABLE 34-Seasonal History of the Codling-moth at Olney, in 1916

\begin{tabular}{|c|c|c|c|c|c|c|c|c|c|c|c|}
\hline Generation & Period & \multicolumn{2}{|c|}{ Earliest } & \multicolumn{2}{|c|}{$\begin{array}{l}\text { Relatively } \\
\text { numerous }\end{array}$} & \multicolumn{2}{|c|}{ Maximum } & \multicolumn{2}{|c|}{$\begin{array}{l}\text { Relatively } \\
\text { numerous }\end{array}$} & \multicolumn{2}{|c|}{ Latest } \\
\hline Hibernating & $\begin{array}{l}\text { Pupæ } \\
\text { Adults }\end{array}$ & $\begin{array}{l}\text { Apri } \\
\text { May }\end{array}$ & $\begin{array}{l}13 \\
11\end{array}$ & $\begin{array}{l}\text { April } \\
\text { May }\end{array}$ & $\begin{array}{l}16 \\
14\end{array}$ & $\begin{array}{l}\text { April } \\
\text { May }\end{array}$ & $\begin{array}{l}20 \\
15\end{array}$ & $\begin{array}{l}\text { May } \\
\text { May }\end{array}$ & $\begin{array}{l}11 \\
27\end{array}$ & $\begin{array}{l}\text { June } \\
\text { June }\end{array}$ & $\begin{array}{l}17 \\
30\end{array}$ \\
\hline First & $\begin{array}{l}\text { Eggs } \\
\text { Young larvæ } \\
\text { Mature larvæ } \\
\text { Pupæ } \\
\text { Adults }\end{array}$ & $\begin{array}{l}\text { May } \\
\text { May } \\
\text { June } \\
\text { June } \\
\text { July }\end{array}$ & $\begin{array}{r}14 \\
25 \\
20 \\
20 \\
3\end{array}$ & $\begin{array}{l}\text { May } \\
\text { May } \\
\text { June } \\
\text { July } \\
\text { July }\end{array}$ & $\begin{array}{r}19 \\
26 \\
29 \\
4 \\
14\end{array}$ & $\begin{array}{l}\text { May } \\
\text { June } \\
\text { July } \\
\text { July } \\
\text { July }\end{array}$ & $\begin{array}{r}24 \\
2 \\
8 \\
15 \\
26\end{array}$ & $\begin{array}{l}\text { June } \\
\text { June } \\
\text { July } \\
\text { Aug. } \\
\text { Aug. }\end{array}$ & $\begin{array}{r}1 \\
9 \\
26 \\
2 \\
10\end{array}$ & $\begin{array}{l}\text { July } \\
\text { July } \\
\text { Aug. } \\
\text { Aug. } \\
\text { Aug. }\end{array}$ & $\begin{array}{c}10^{*} \\
18^{*} \\
9 \\
14 \\
24^{*}\end{array}$ \\
\hline Second & $\begin{array}{l}\text { Eggs } \\
\text { Young larvæ } \\
\text { Mature larvæ } \\
\text { Pupæ } \\
\text { Adults }\end{array}$ & $\begin{array}{l}\text { July } \\
\text { July } \\
\text { July } \\
\text { Aug. } \\
\text { Aug. }\end{array}$ & $\begin{array}{r}6 \\
12 \\
31 \\
3 \\
12\end{array}$ & $\begin{array}{l}\text { July } \\
\text { July } \\
\text { Aug. } \\
\text { Aug. } \\
\text { Aug. }\end{array}$ & $\begin{array}{r}15 \\
21 \\
8 \\
11 \\
15\end{array}$ & $\begin{array}{l}\text { July } \\
\text { Aug. } \\
\text { Sept. } \\
\text { Aug. } \\
\text { Aug. }\end{array}$ & $\begin{array}{r}26 \\
1 \\
10 \\
17 \\
26\end{array}$ & $\begin{array}{l}\text { Aug. } \\
\text { Aug. } \\
\text { Sept. } \\
\text { Sept. } \\
\text { Sept. }\end{array}$ & $\begin{array}{r}13 \\
21 \\
25 \\
22 \\
7\end{array}$ & $\begin{array}{l}\text { Sept. } \\
\text { Sept. } \\
\text { Oct. } \\
\text { Sept. } \\
\text { Sept. }\end{array}$ & $\begin{array}{c}4 \\
21 \\
23 \dagger \\
3 \\
14^{*}\end{array}$ \\
\hline Third & $\begin{array}{l}\text { Eggs } \\
\text { Young larvæ } \\
\text { Mature larvæ }\end{array}$ & $\begin{array}{l}\text { Aug. } \\
\text { Aug. } \\
\text { Oct. }\end{array}$ & $\begin{array}{l}14^{*} \\
20^{*} \\
1\end{array}$ & $\begin{array}{l}\text { Aug. } \\
\text { Aug. }\end{array}$ & $\begin{array}{l}18 \\
28\end{array}$ & $\begin{array}{l}\text { Aug. } \\
\text { Sept. }\end{array}$ & $\begin{array}{l}29 \\
12\end{array}$ & $\begin{array}{l}\text { Sept. } \\
\text { Sept. }\end{array}$ & $\begin{array}{l}10 \\
13\end{array}$ & $\begin{array}{l}\text { Sept. } \\
\text { Sept. } \\
\text { Oct. }\end{array}$ & $\begin{array}{l}21 \\
15 \\
23 \dagger\end{array}$ \\
\hline
\end{tabular}

* Dates computed.

† Last observation. 


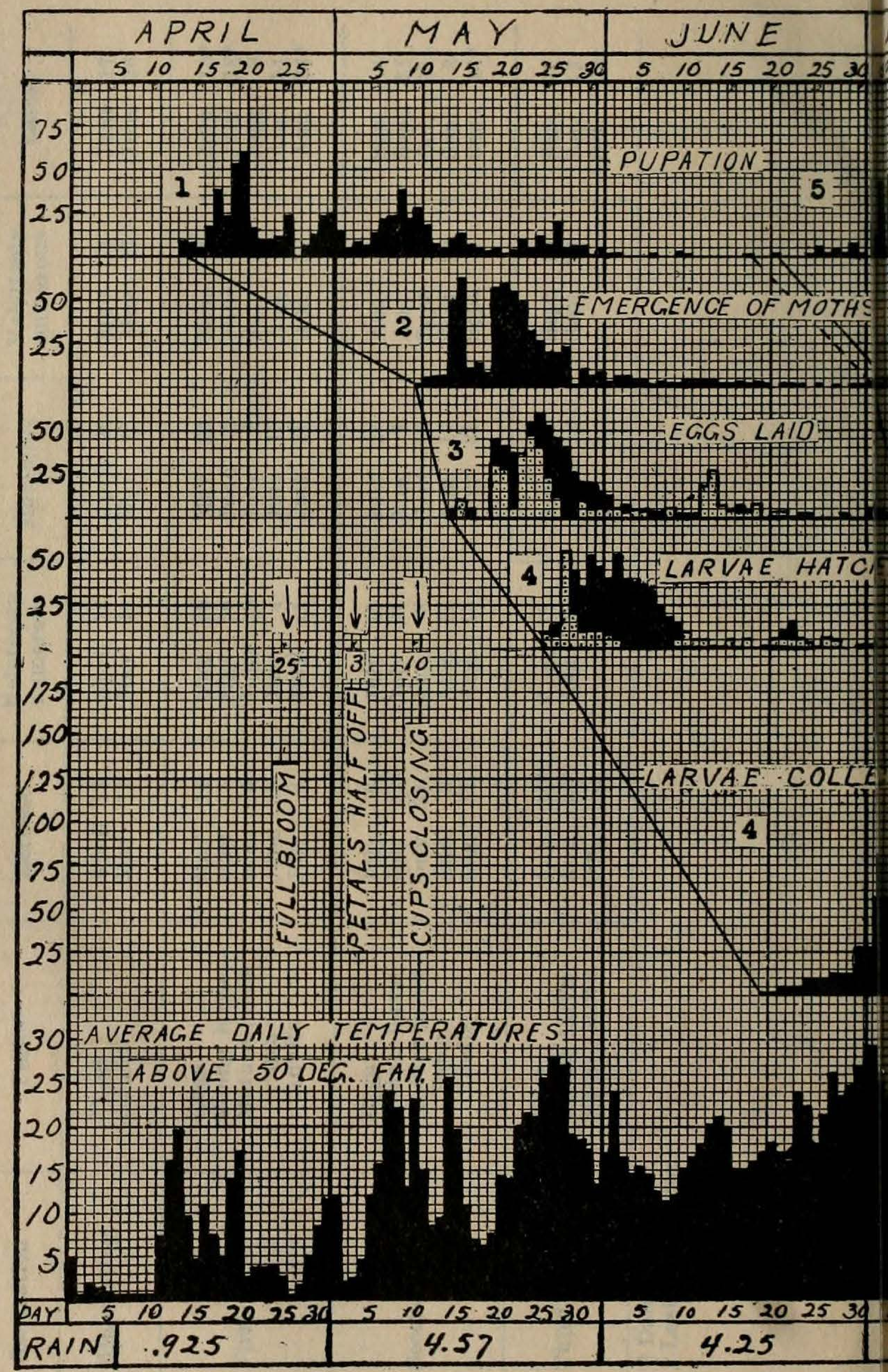

Chart 2. Seasonal histor 


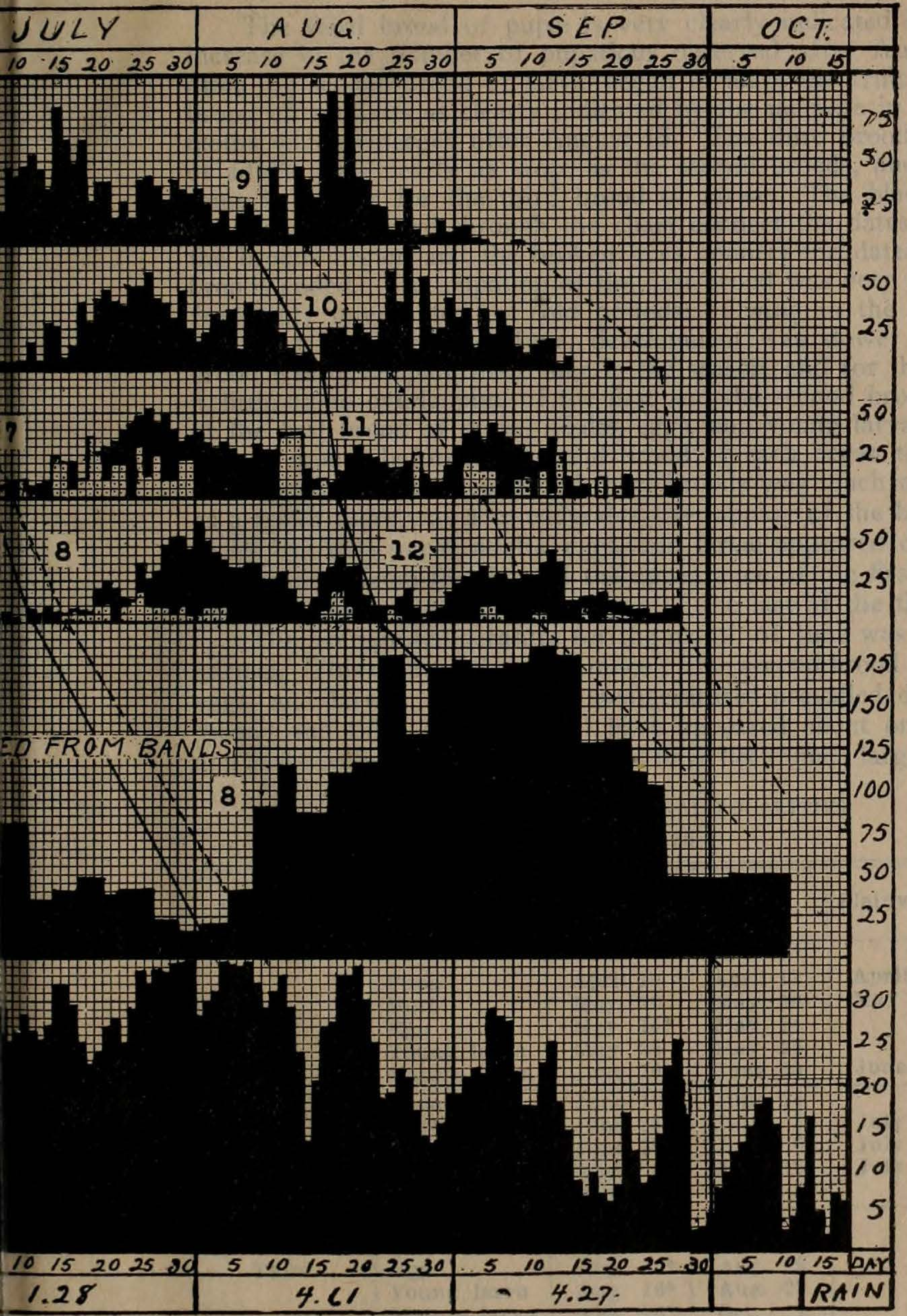

: codling-moth at Olney, 1916. 
The third brood of pupæ is very clearly indicated by the marked increase in the number of pupations observed after August 8 or just after the second brood of larvæ began to leave the fruit, and the third brood of adults is as clearly indicated by the increase in the number of noths which emerged after August 15. The third broods of pupæ and of adults were nearly as large as the second broods, and no doubt the same held true for the third brood of larvæ. The black lines drawn obliquely downward through the chart connect the dates when each of the broods began, and the broken lines connect the dates on which the broods ended. The pupation of the first brood was very irregular, being frequently interrupted by cold periods. Owing to the cooler weather during April, May, and June, development was slower while the first brood was developing than later in the season, and for this reason there is little or no overlapping of the first and the second broods. The bulk of the first brood of pupæ, adults, eggs, and young larvæ had appeared fully a month before the second brood of each began to appear. The development of the second and third broods was much more rapid, and as a result there was a considerable overlapping of the broods, so much so in fact that pupæ, adults, eggs, and larvæ appeared daily, with little interruption, from the date of the appearance of the first of the second brood to the date of the appearance of the last of the third brood.

The average temperature for the month of June was about $5^{\circ}$ below normal. This delayed the appearance of the second brood of pupæ, adults, eggs, and larvæ. Had normal temperatures prevailed during June the second and third broods would have appeared about one week earlier, and as a result the third brood would have been much larger.

RECORDS FOR SEVERAL LOCALITIES

TABLE 35

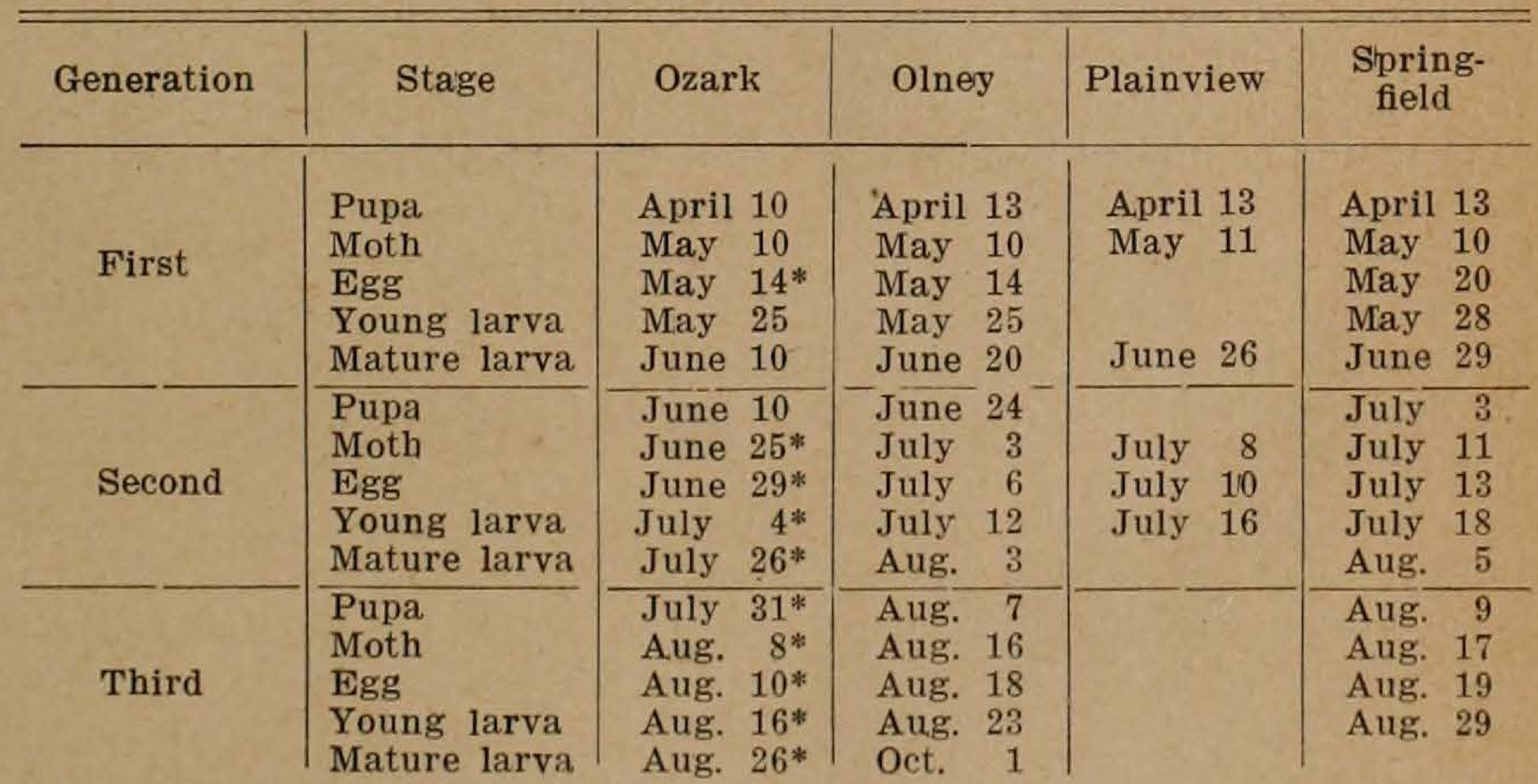

* Dates followed by an asterisk were computed. 
Dates of the appearance of the different stages at Olney and other points in 1916 are given in Table 35 , immediately preceding.

Olney is 75 miles, Plainview 108 miles, and Springfield 150 miles north of Ozark. The overwintering larvæ began to pupate at Ozark April 10, at Olney, Plainview, and Springfield April 13. The first moths emerged and the first eggs hatched on nearly the same dates at the four places. Larvæ of the second generation began to hatch about 8 days later at Olney, 12 days later at Plainview, and 14 days later at Springfield than at Ozark, and larvæ of the third generation began to hatch 7 days later at Olney and 13 days later at Springfield than at Ozark. Three generations were recorded at all four points. The third generation at Springfield was small as compared with the third generation at Olney.

\section{Seasonal History of the Codling-moth, 1917}

The methods of work pursued during 1915 and 1916 were continued in 1917. The work at Olney was closed in July, so complete records were not kept of the second and third generations at that place.

The season was not favorable for rapid development. While the mean temperature for April was about normal the temperature during the first half of the month was much below normal. May and June were cold months. The total accumulation of monthly degrees above 50 for April, May, June, July, and August was 86.9, the normal being 93.3.

\section{LARGE-CAGE SERIES}

First generation.-The material collected from cages in 1916 was not preserved, hence no record of the pupation and emergence of hibernating material was secured from cages. Hibernating larvæ collected from bands in 1916 began to pupate April 3. Moths began to emerge May 17 and continued to emerge till June 24. The first moths to emerge were not liberated in a large cage because the dates when the first eggs, larvæ, pupæ, and adults of the first generation appeared could be easily observed without using a cage; but the last moths to emerge from the hibernating band material were liberated in a large screen-cage to get the dates when the last eggs, larvæ, pupæ, and adults of the first generation appeared.

The first eggs of the first generation were laid May 19 and hatched June 1. The first mature larvæ left the fruit June 23 and pupated June 26 , and the first moths emerged July 7 . The last eggs of the first generation were noted in the large cage June 27. They were probably laid two or three days earlier. They hatched July 1. Mature larvæ were found under the band on the tree in this cage from July 13 to August 13. No observations were made to ascertain the dates of the last pupæ and adults. 
Second generation.-The first moths of the first generation were liberated in cage No. 2 to rear the first individuals of the second genera tion.

Moths were liberated in cage No. 2, as follows:

\begin{tabular}{crcc}
\multicolumn{2}{c}{ Date } & Males & Females \\
July & 8 & 1 & 1 \\
“ & 9 & 1 & 1 \\
“" & 10 & 4 & 3 \\
“" & 11 & 4 & 1 \\
“" & 13 & 8 & 1 \\
“ & 14 & 9 & 6 \\
& 14 & 9 & 12
\end{tabular}

Eggs were found July 11 and young larvæ July 17 . Daily observations ceased before larvæ began to leave the fruit in this cage.

Third generation.-The first eggs probably were laid about August 16 and hatched about August 22.

\section{BAND COLLECTIONS}

Hibernating generation, 1916-1917- - Of the 2687 adults of the hibernating generation emerging in 1917, 1258 were males and 1429 were females (Table 36). The average date of the emergence of the males was May 25, of the females May 27, and of the entire brood May 26. This was five days after the date of the maximum emergence. There was practically no difference between the dates of emergence of moths from larvæ which left the fruit in August and the emergence of those from larvæ which left the fruit later.

Collections made in 1917 (see Table 37).-The first mature larvæ were taken under bands at Springfield only two days later than at Olney. The second generation of larvæ began to leave the fruit at Olney between July 23 and July 30, but, unfortunately, there was a period of seven days here between the records, and the exact date can not be accurately determined. The bands at Springfield were torn from the trees some time between August 4 and August 12 by some one unknown, and hence the date when the second generation began to leave the fruit at Springfield is also uncertain.

-The falling off in the number of larvæ taken at Springfield after October 6 was due largely to the fact that the apples were picked from half of the trees soon after that date.

Complete records of pupation, and the emergence of moths from band material were not preserved. At Ozark the first larvæ left the apples June 18 and the first moth emerged July 1. At Olney the first larvæ left the fruit June 23, and pupated June 26, the first moths emerging July 7. At Springfield the first larvæ left the fruit June 25, and pupated June 28 , the first moths emerging July 9. (Table 40, p. 277.) 
Observations ceased too early to get complete data on pupation and emergence of the second generation, or on the third generation. There is no doubt, however, that there was a third generation at Olney. Larvæ of the second generation began to leave the fruit about August 4, and since transforming of larvæ usually continues till the end of August, no doubt many of the first larvæ of the second generation transformed and produced a partial third generation. The first eggs of the third generation were laid about August 23 and hatched about August 31.

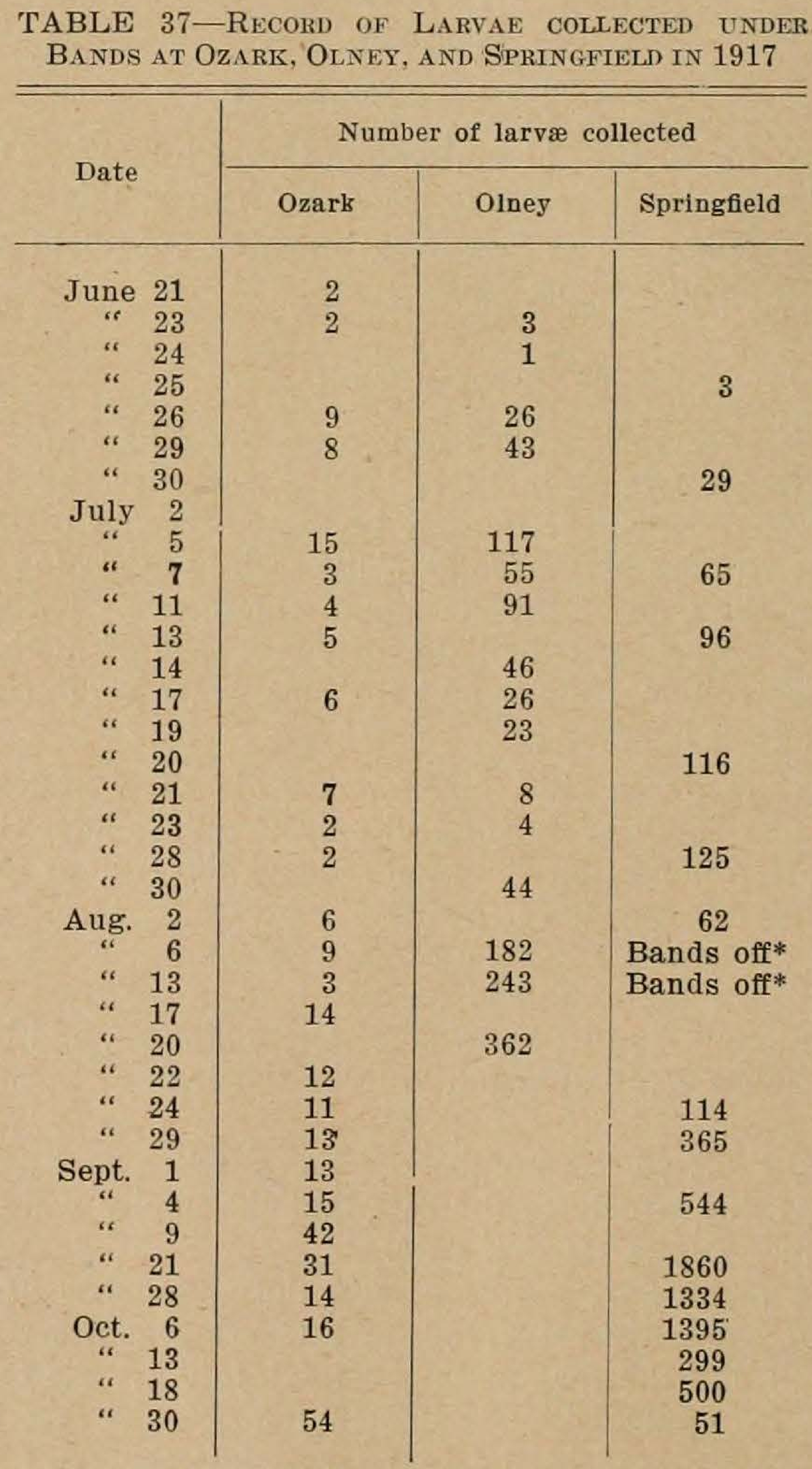

* See p. 273 , line 13 from bottom. 


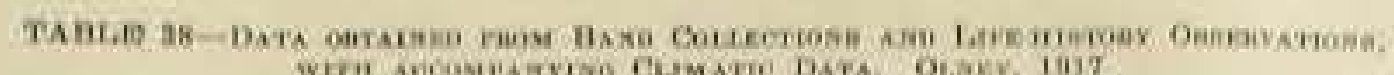

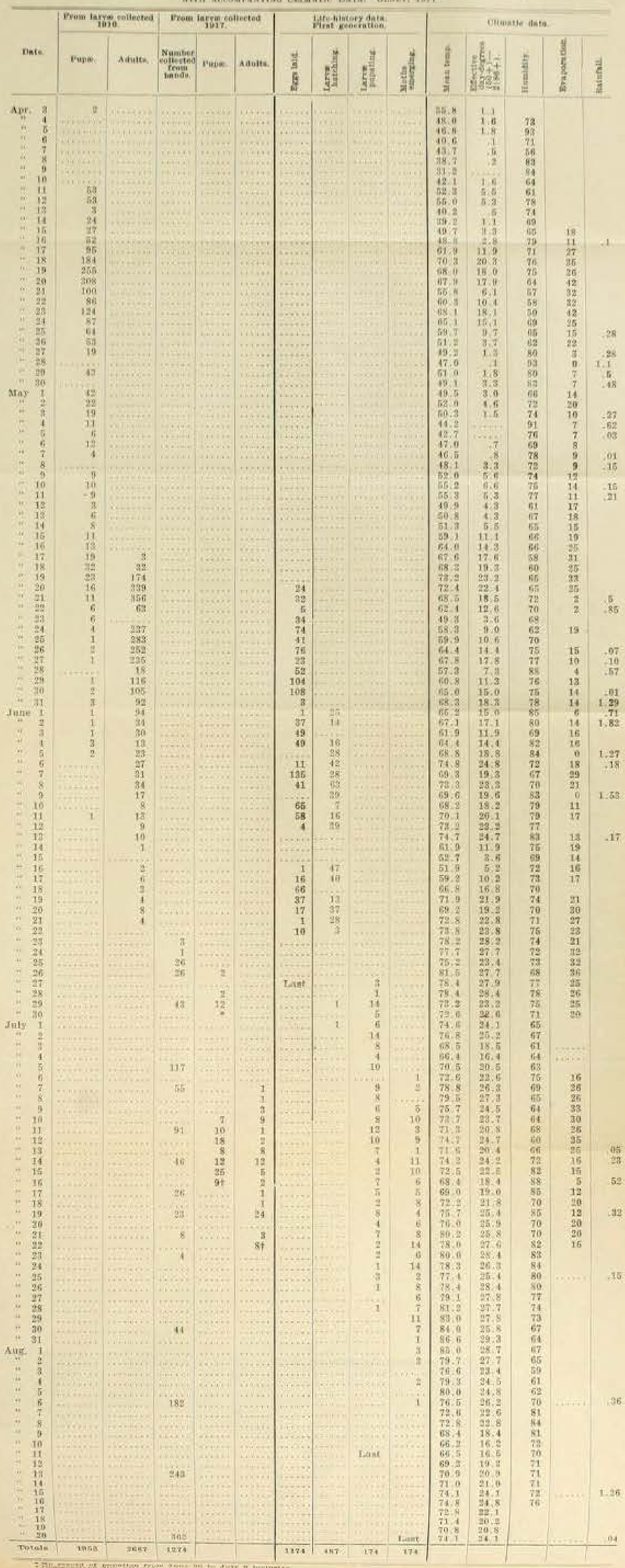


A daily record of the band collections, life-history observations, and climatic conditions at Olney in 1917 have been brought together in Table 38 for ready reference.

\section{SUMMARY OF THE SEASONAL HISTORY, 1917}

From the foregoing data, the seasonal history of the codling-moth at Olney can be quite accurately determined (Table 39).

Chart 3 represents in graphic form, the data given in Table 39. These data are disconnected and meager for the second and third generations. The position and form of the curved lines in the chart, representing the relative number of eggs and young larvæ of the first generation and the number of the different stages of the second and third generations, are based upon estimates only, since complete daily records were not kept.

The bulk of the hibernating larvæ pupated during the unusually warm period in the third week of April; the bulk of the adults emerged during the last two weeks of May; the bulk of the eggs of the first generation were laid during the last ten days of May and the first week in June; and the bulk of the larvæ hatched during the first three weeks of June. There was no overlapping of the first and second generations except in the larval stage.

\section{Relation of Temperature to the Seasonal History OF THE CODLING-MOTH}

It has already been shown that there is quite a constant relation between the temperatures above 50 degrees when corrected for retarding temperatures above 86 degrees and the length of time required to bring the insect through the egg, larval, and pupal periods. This applies to all the periods except the larval period of the hibernating larvæ. The length of time the hibernating larvæ remain in the cocoon is independent of the day-degrees which accumulate between the time when hibernation begins and the winter months. It is found, however, that there is a more or less definite relation between the average daily temperatures above 50 degrees in the spring months and the dates when the first pupæ and the first adults appear. There is a very great individual variation in this respect, as will be seen by referring to the last column of Tables 19 and 20.* In Table 19, it will be seen that the hibernating larvæ began to pupate when the sum of the day-degrees above 50 degrees $\mathrm{F}$. after January 1 amounted to 115 ; the accumulations for the others ranged from 115 to 356 , the average being 186 . In Table 20 it will be seen that the hibernating larvæ of 1916-1917 began to pupate when the sum of the day-degrees above 50 degrees $F$. amounted to 104 . The others varied from 104 to 355 , the average being 190 .

\footnotetext{
*Tables 9-25 follow p. 253.
} 
TABLE 39-Seasonal History of the Coding-moth at OLney, 1917

\begin{tabular}{|c|c|c|c|c|c|c|}
\hline Generation & Period & Earliest & $\begin{array}{l}\text { Relatively } \\
\text { numerous }\end{array}$ & Maximum & $\begin{array}{l}\text { Relatively } \\
\text { numerous }\end{array}$ & Latest \\
\hline Hibernating & $\begin{array}{l}\text { Pupæ } \\
\text { Adults }\end{array}$ & $\begin{array}{l}\text { April } 3 \\
\text { May } 17\end{array}$ & $\begin{array}{l}\text { April } 11 \\
\text { May } 19\end{array}$ & $\begin{array}{l}\text { April } 20 \\
\text { May } 21\end{array}$ & $\begin{array}{l}\text { April } 26 \\
\text { June } 1\end{array}$ & $\begin{array}{l}\text { June } 11 \\
\text { June } 21\end{array}$ \\
\hline First & $\begin{array}{l}\text { Eggs } \\
\text { Young larvæ } \\
\text { Mature larvæ } \\
\text { Pupæ } \\
\text { Adults }\end{array}$ & $\begin{array}{lr}\text { May } & 20 \\
\text { June } & 1 \\
\text { June } & 23 \\
\text { June } & 27 \\
\text { July } & 7\end{array}$ & $\begin{array}{lr}\text { May } & 21 \\
\text { June } & 2 \\
\text { June } & 26 \\
\text { June } & 30 \\
\text { July } & 10\end{array}$ & $\begin{array}{lc}\text { May } & 25 \\
\text { June } & 8^{*} \\
\text { July } & 6 \\
\text { July } & 10^{*} \\
\text { July } & 20^{*}\end{array}$ & $\begin{array}{lc}\text { June } & 3 \\
\text { June } & 10 \\
\text { July } & 19 \\
\text { July } & 22 * \\
\text { Aug. } & 2\end{array}$ & $\begin{array}{lr}\text { June } & 27 \\
\text { July } & 1 \\
\text { Aug. } & 6^{*} \\
\text { Aug. } & 11^{*} \\
\text { Aug. } & 20^{*}\end{array}$ \\
\hline Second & $\begin{array}{l}\text { Eggs } \\
\text { Young larvæ } \\
\text { Mature larvæ } \\
\text { Pupæ } \\
\text { Adults }\end{array}$ & $\begin{array}{l}\text { July } 11 \\
\text { July } 17 \\
\text { Aug. } 4 * \\
\text { Aug. } 8^{*} \\
\text { Aug. } 21^{*}\end{array}$ & & - & & Aug. 23* \\
\hline Third & $\begin{array}{l}\text { Eggs } \\
\text { Young larvæ }\end{array}$ & $\begin{array}{l}\text { Aug. 23* } \\
\text { Aug. } 31^{*}\end{array}$ & & & & \\
\hline
\end{tabular}

* Computed. 


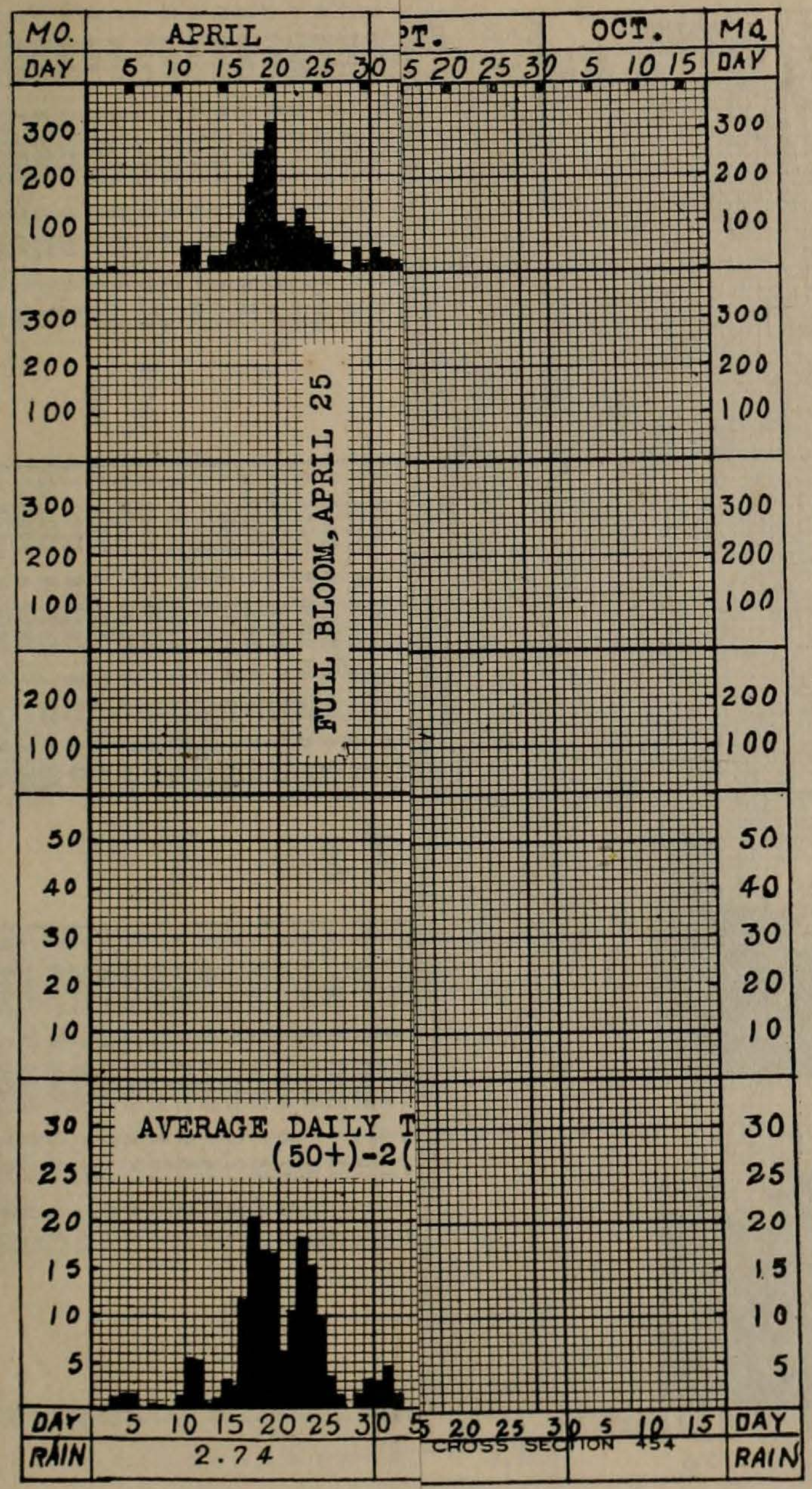




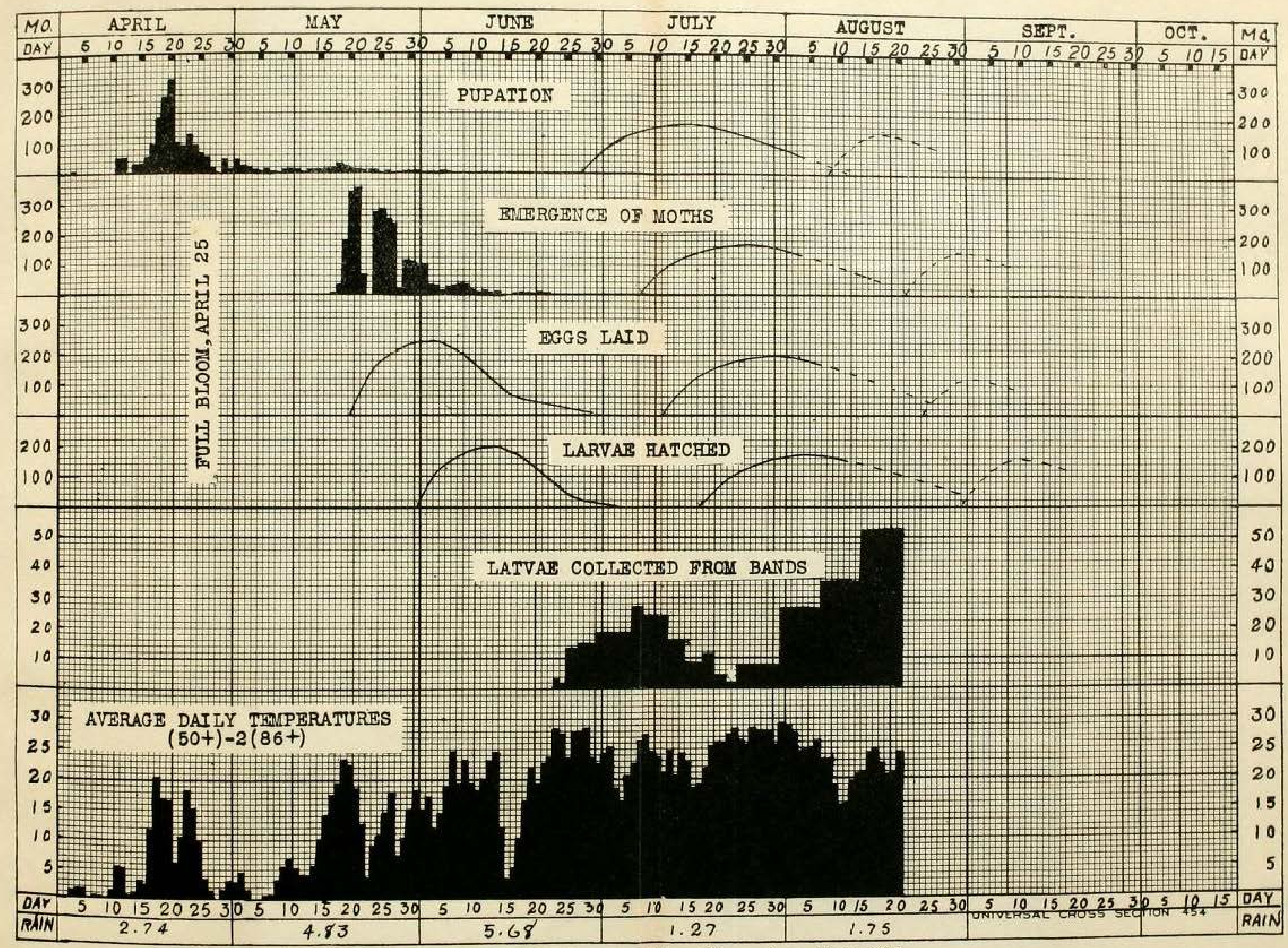

CHART 3. Seasonal history of the codling-moth at Olney, 1917. 


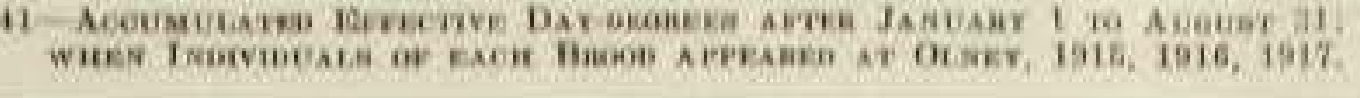

\begin{tabular}{|c|c|c|c|c|c|c|c|c|c|c|c|c|c|c|c|}
\hline \multirow{3}{*}{ Doste } & \multicolumn{5}{|c|}{1015} & \multicolumn{5}{|c|}{6810.} & \multicolumn{5}{|c|}{$1617 \%$} \\
\hline & \multirow[b]{2}{*}{ stake } & \multicolumn{2}{|c|}{$\begin{array}{l}\text { Dalis } \\
\text { danxdogerese }\end{array}$} & \multicolumn{2}{|c|}{ 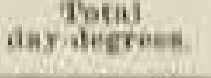 } & \multirow[b]{2}{*}{$\operatorname{stagen}$} & \multicolumn{2}{|c|}{$\begin{array}{l}\text { Daily } \\
\text { any deskrews }\end{array}$} & \multicolumn{2}{|c|}{$\begin{array}{l}\text { Total } \\
\text { ing fleareen }\end{array}$} & \multirow[b]{2}{*}{ Striav: } & \multicolumn{2}{|c|}{$\begin{array}{l}\text { balls } \\
\text { any dekrenten }\end{array}$} & \multicolumn{2}{|c|}{$\begin{array}{l}\text { Totai } \\
\text { Asycidgreten }\end{array}$} \\
\hline & & $\frac{7}{8}$ & 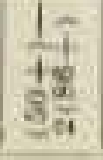 & 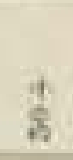 & $\frac{15}{\frac{1}{8}}$ & & $\frac{1}{3}$ & $\frac{\frac{1}{1}}{\frac{1}{8}}$ & $\frac{1}{3}$ & $\frac{-7}{4 x}$ & & $\frac{1}{5}$ & $\frac{1}{5}$ & a & $\frac{1}{\frac{1}{3}}$ \\
\hline
\end{tabular}

Mar,
Apr.

Pupa

\begin{tabular}{ll|l|l|}
\hline 2.1 & 12.1 & 66.7 & 66.7 \\
\hline 4.3 & 14.3 & 71.0 & 71.
\end{tabular}

\begin{tabular}{|r|r|r|r|}
\hline 16.7 & 16.7 & 87.7 & 87.7 \\
\hline 10.1 & 10.1 & 97.8 & 97.8 \\
5.1 & 5.1 & 103.9 & 102.9 \\
\hline & 6 & 103.5 & 103. \\
\hline
\end{tabular}

\begin{tabular}{l|l|l|l|}
1.6 & 1.6 & 105.1 & 105.1 \\
4.7 & 1.7 & 109.8 & 109.8 \\
1.8 & 5.8 & 115.8 & 15.8
\end{tabular} \begin{tabular}{lllll}
11.3 & 11.3 & 126.9 & 126. \\
\hline
\end{tabular} \begin{tabular}{cccc}
10.0 & 10.0 & 1.66 .9 & 136.9 \\
\hline
\end{tabular} \begin{tabular}{|l|l|l|l|}
\hline 14.2 & 14.2 & 160.6 & 160. \\
15.8 & 15.8 & 176.4 & 176 \\
14.6 &
\end{tabular} $\begin{array}{llll}14.9 & 14.9 & 191.3 & 191.3 \\ 14.1 & 14.1 & 205.4 & 20.5\end{array}$ \begin{tabular}{|l|l|l|l|}
\hline 19.0 & 19.0 & 234.4 & 224.4 \\
22.7 & 22.7 & 247.1 & 247.1 \\
\hline
\end{tabular}

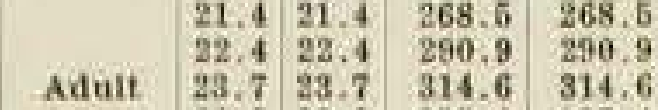

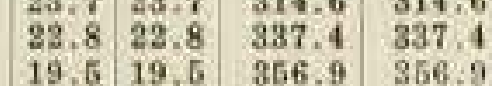

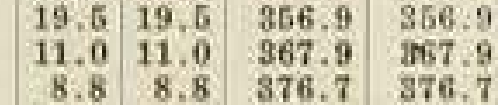

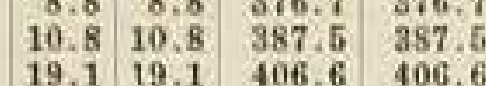

Egg \begin{tabular}{|lll|l|}
8.4 & 8.4 & 415.0 & 415.0 \\
6.8 & 6.8 & 421.8 & 421.8 \\
9.0 & 9.0 & 430.8 & 430.8 \\
\hline & & &
\end{tabular} \begin{tabular}{rrr|r}
9.0 & 9.0 & 430.8 & 430.8 \\
12.1 & 12.1 & 44.9 & 42.9 \\
7 & 7.5 & 45.2 & 45.9
\end{tabular} \begin{tabular}{lll|l|l|}
6.2 & 5.2 & 455.6 & 456 \\
7.0 & 7.0 & 462.6 & 462. \\
\hline & 0.0 & &
\end{tabular}

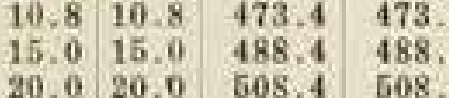
\begin{tabular}{|l|l|l|l|}
\hline 20.0 & 200.0 & 580.4 & 508 \\
23.0 & 23.0 & 531.4 & 531 \\
\hline 2.5 &
\end{tabular}

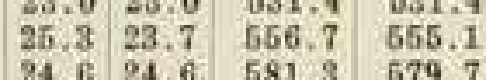

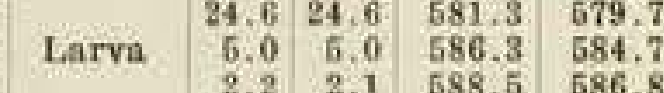
\begin{tabular}{llll|l|}
2.2 & 2.1 & 588.5 & 586.8 \\
1.4 & 1.4 & 589.9 & 588.8 \\
\hline
\end{tabular} \begin{tabular}{|r|r|r|r|}
1.4 & 1.4 & 589.9 & 588.2 \\
10.4 & 10.4 & 600.3 & 598.6 \\
\hline 1.0 & 0.4 & 621.5 & 569.8
\end{tabular} $\begin{array}{llll}11.0 & 21.0 & 621.3 & 619.6 \\ 15.3 & 15.3 & 636.6 & 634.8 \\ 11.0 & 11.0 & 647.8 & 0.5\end{array}$ $\begin{array}{lllll}16.0 & 16.0 & 647.6 & 645 . \\ 16.8 & 661.9\end{array}$ \begin{tabular}{|l|l|l|l|}
\hline 15.5 & 15.5 & 679.1 & 677.4 \\
14.1 & 14.1 & 693.2 & 691.6 \\
\hline 13.5 & 13.5 & 713.8 &
\end{tabular} \begin{tabular}{|c|c|c|c|}
\hline 14.3 & 14.1 & 69.2 & 691.5 \\
7.3 & 7.3 & 700.5 & 698.8 \\
\hline
\end{tabular} \begin{tabular}{|l|l|l|l|}
\hline 13.3 & 13.3 & 713.8 & 712.1 \\
12.8 & 12.8 & 726.6 & 724.9 \\
\hline 1.8 & 19.8 & 79.4 & 74.8
\end{tabular} \begin{tabular}{rrrr|r}
8.8 & 8.8 & 735.4 & $733 . ?$ \\
12.3 & 12.3 & 747.7 & 746.8
\end{tabular} \begin{tabular}{|l|l|l|l|}
\hline 14.8 & 14.8 & 762.5 & 760.8 \\
15.6 & 15.6 & 778.1 & 776.4 \\
\hline
\end{tabular} $\begin{array}{llll}17.1 & 21.1 & 816.5 & 814.8 \\ 23 & 83.1 & 0.8\end{array}$

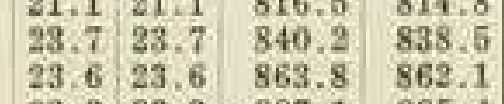

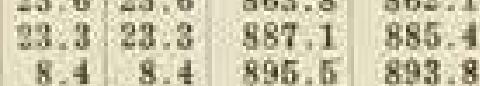

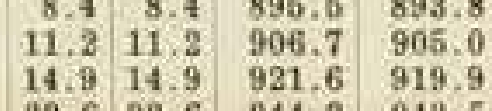

Mature
larva 23.6 .

Pupa

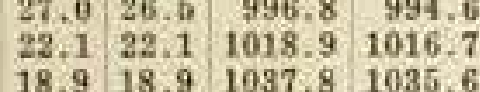

$\begin{array}{lllll}18.8 & 18.8 & 1056.6 & 1054 .\end{array}$ $\begin{array}{llll}22.6 & 22.6 & 1100.0 & 1097.8 \\ 21 & & \end{array}$ $\begin{array}{llll}22.6 & 2.6 & 1121.6 & 1119 \\ 23.6 & 23.6 & 1145.2 & 1143\end{array}$

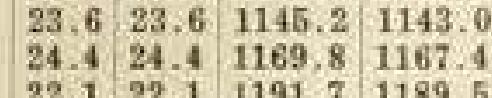
$\begin{array}{lllll}17.8 & 17.8 & 1209.5 & 1207.3\end{array}$

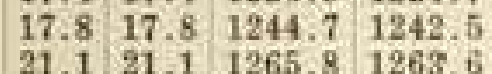

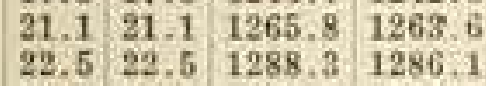

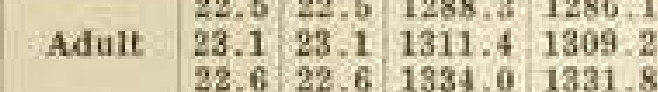
\begin{tabular}{|lllll}
21.5 & 21.5 & 1355 & 1363.3 \\
21.1 & 21.1 & 1376.6 & 1374. \\
\hline
\end{tabular}

Esg $\begin{array}{lllll}19.5 & 19.5 & 1417.6 & 1415.4 \\ 15.2 & 15.2 & 1432.8 & 1430\end{array}$ 11.111 .1 1443.9 1441. $\begin{array}{llll}19.4 & 19.4 & 1478.9 & 1476\end{array}$ $\begin{array}{lllll}18 . & 18.4 & 1497.3 & 1495 . \\ 18.3 & 18.3 & 1516.6 & 1513 .\end{array}$ Larvi $\begin{array}{lllll}23.7 & 23.7 & 1569.5 & 1557 . \\ 23.753 .2 & 1581 .\end{array}$ $\begin{array}{llll}27.4 & 27.1 & 1610.6 & 1608 .\end{array}$ $\begin{array}{llll}30.3 & 28.8 & 1640.9 & 1636.9 \\ 32.6 & 30.3 & 1673.5 & 1667 . \\ 3 & 39.8 & 1736.9 & 198 .\end{array}$ $32.3 \quad 30.61706 .81697$ $\begin{array}{lllll}30.5 & 29.7 & 1766.5 & 1757.2\end{array}$ $\begin{array}{lllll}26.6 & 26.6 & 1820.3 & 1811 .\end{array}$ 18.9 18.9 1859.2 1829 $20.2 \quad 20.2 \quad 1878.2 \quad 1868$ $\begin{array}{lllll}21.3 & 21.3 & 1899.5 & 1890.2\end{array}$ $\begin{array}{lllll}22.4 & 22.4 & 1921.9 & 1912 . \\ 25.5 & 25.5 & 1947.4 & 1938 .\end{array}$ 27.4 27.4 $1974.8 \quad 1965$. $29.129 .12031 .1 \quad 2020$. Mature
larva $\begin{array}{llll}32.0 & 29.5 & 2092.4 & 2079.4 \\ \text { larva }\end{array}$

\begin{tabular}{rrrrr}
27.3 & 27.3 & 2119.7 & 2106.7 \\
\hline & 26.8 & 26.8 & 2146.5 & 2133.5 \\
\hline
\end{tabular} $\begin{array}{llllll}26.8 & 26.8 & 2146.5 & 2185 . \\ 25.8 & 25.8 & 2172.3 & 2159 .\end{array}$ \begin{tabular}{|lll|l|l|}
\hline 15.1 & 15.1 & 2187.4 & 2174. \\
13.8 & 13.8 & 2201.2 & 2188.
\end{tabular} 20.1 20.1 2221.32208 . $\begin{array}{lllll}20.4 & 20.4 & 2241.7 & 2228 . \\ 36-1 & 26.1 & 2267.8 & 2254\end{array}$ $\begin{array}{llll}22.7 & 22.7 & 2315.8 & 2302.8\end{array}$ \begin{tabular}{lllll}
18.8 & 18.8 & 2334.6 & 2321 \\
18.8 & 18.2 & 2352.8 & 2339 \\
\hline
\end{tabular}

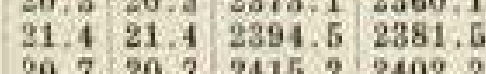
$\begin{array}{lllll}27.3 & 20.7 & 2415,2 & 2402 . \\ 2442.6 & 2429 .\end{array}$ 7.5
16.7
16.5

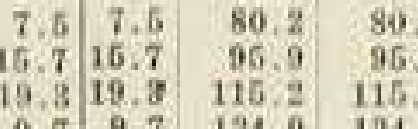
\begin{tabular}{r|ccc}
9.7 & 9.7 & 12.9 & 124. \\
4.3 & 4.3 & 129.2 & 129 \\
\hline & 9.9 & &
\end{tabular} $\begin{array}{llll}10.9 & 10.9 & 140.1 & 140\end{array}$ 4.8 4.8 152.1 152. \begin{tabular}{l|lll|l}
7.3 & 17.3 & 183.2 & 183 \\
2.3 & 2.3 & 185.5 & 186 \\
3.8 & 3.8 & 195. &
\end{tabular} \begin{tabular}{l|l|l|l|}
3.8 & 3.8 & 193.1 & 193 \\
\hline
\end{tabular} \begin{tabular}{|l|ll|l|}
\hline 3.3 & 3.2 & 196.4 & 196. \\
2.6 & 2.6 & 199.0 & 199. \\
\hline & & &
\end{tabular} \begin{tabular}{l|lll}
1.4 & 1.4 & 200.7 & 200 \\
\hline 5.2 & 8 & 205 & 205
\end{tabular} \begin{tabular}{|c|c|c|c|}
\hline 5.0 & 5.0 & 205.7 & 205.7 \\
8.3 & 8.3 & 214.0 & 214 \\
\hline
\end{tabular}

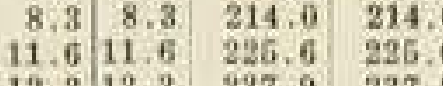
\begin{tabular}{rr|r|r|r}
12.3 & 12.3 & 237.9 & 237 \\
1.8 & 1.8 & 239.7 & 239 \\
\hline
\end{tabular} \begin{tabular}{rrrr}
3.2 & 2.2 & 241.9 & 24.9 \\
4.6 & 4.6 & 246.5 & 24 \\
\hline & &
\end{tabular}

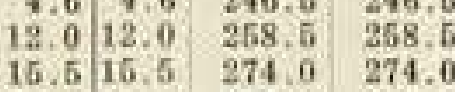
$\begin{array}{llll}24.0 & 24.0 & 298.0 & 298 \\ 23.0 & 22.0 & 320.0 & 320\end{array}$

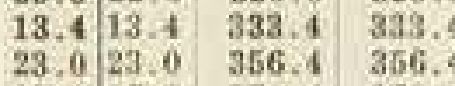
$\begin{array}{lllll}8.5 & 8.6 & 379.9 & 371 \\ 879 & \end{array}$ $\begin{array}{lllll}9.5 & 9.5 & 389.4 & 389.4\end{array}$

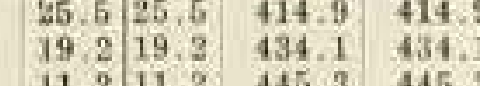
\begin{tabular}{l|lll}
5.8 & 5.8 & 451,1 & 451
\end{tabular} \begin{tabular}{|l|l|l|l|}
5.5 & 5.5 & 456.6 & 456.6 \\
8.4 & 8.4 & 465.0 & 465.0
\end{tabular} \begin{tabular}{llll}
14.1 & 14.1 & 479.1 & 479.1 \\
13.13 & 13.9 & 493 & 493.0 \\
\hline
\end{tabular} $20.490 .4513 .4 \quad 513.4$ $\begin{array}{llll}21.3 & 21.3 & 556.2 & 556.2\end{array}$

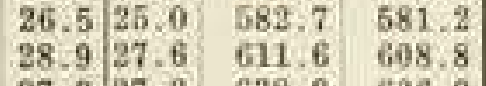

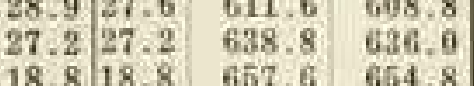
\begin{tabular}{cc|c|c|}
18.8 & 18.8 & 657.6 & 654.8 \\
18.6 & 18.6 & 676.2 & 673.4 \\
\hline
\end{tabular} $\begin{array}{lllll}18.6 & 18.6 & 676.2 & 673 . \\ 17.8 & 17.8 & 694.0 & 691\end{array}$ \begin{tabular}{llll}
17.0 & 24,1 & 748.6 & 745 \\
\hline
\end{tabular} \begin{tabular}{|l|l|l|l|}
\hline 24.1 & 17.0 & 724.5 & 721. \\
\hline 16.3 & 16.3 & 764.9 & 762. \\
\hline
\end{tabular} $\begin{array}{llll}14.0 & 14.0 & 78.9 & 776 .\end{array}$ \begin{tabular}{|l|l|l|l|}
\hline 15.6 & 15.6 & 794.5 & 791. \\
\hline 14.6 & 14.5 & 809.0 & 806 \\
\hline
\end{tabular}

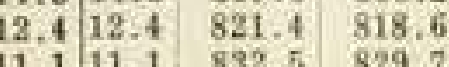
\begin{tabular}{|l|l|l|l|}
\hline 11.2 & 11.7 & 832.6 & 829.7 \\
11.2 & 11.7 & 844.2 & 841.4 \\
15.2 & 15.2 & 859.4 & 856.6 \\
\hline
\end{tabular} \begin{tabular}{l|l}
.6 & 872.8
\end{tabular} \begin{tabular}{|l|l|l|l|}
\hline 18.0 & 18.0 & 893.6 & 890.8 \\
20.2 & 20.2 & 913.8 & 911.0 \\
\hline
\end{tabular}

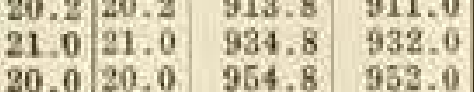
Mature
larva \begin{tabular}{l|l|l|l}
15.0 & 15.0 & 969.8 & 967.0
\end{tabular} \begin{tabular}{llll}
16.6 & 16.6 & 1001.4 & 998.6 \\
\hline
\end{tabular} 17. 017.0 1018.4 1015. $\begin{array}{llll}17.4 & 17.4 & 1054.3 & 1051.5 \\ 16.6 & 16.6 & 1070.9 & 1068.1\end{array}$ \begin{tabular}{|l|l|l|l|}
22.4 & 22.4 & 1093.3 & 1090.5 \\
\hline
\end{tabular} 18.8 18.8 1134.41131 .6 21.631 .61156 .0001153$.

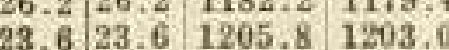
\begin{tabular}{ll|lll}
24.9 & 24.9 & 1230.7 & 1227.9 \\
27.8 & 25.5 & 1258.6 & 1253.4
\end{tabular}

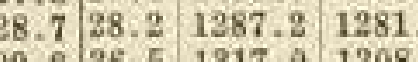
$30.329 .0 \quad 1347.31337$. \begin{tabular}{l|llll}
25.1 & 25.1 & 1372.4 & 1362. \\
23.6 & 23.6 & 1396.0 & 1385.
\end{tabular} $\begin{array}{lllll}23.9 & 23.9 & 1419.9 & 140 . \\ 26.8 & 25.3 & 1446.7 & 1436 \\ 28 & & \end{array}$ $\begin{array}{lllll}28.2 & 26.2 & 1474.9 & 1461 . \\ 25.0 & 25.0 & 1499.9 & 1486 .\end{array}$ \begin{tabular}{lllll}
28.4 & 26.1 & 1554.5 & 1539. \\
26.5 & 27.5 & 1581.0 & 1566 \\
\hline
\end{tabular} \begin{tabular}{l|lll}
26.5 & 26.5 & 1581.0 & 1566. \\
25.7 & 25.4 & 1606.7 & 1591 \\
31.5 & 28.5 &
\end{tabular} $\begin{array}{llll}31.6 & 28.6 & 1665.5 & 1647\end{array}$ $\begin{array}{lllll}32.2 & 28.9 & 1697.7 & 1676 .\end{array}$ $\begin{array}{lllll}31.1 & 37.4 & 1728.8 & 1708 \\ 31.3 & 28.4 & 1760.1 & 1731\end{array}$ 30.1 28.61790 .21760$. $\begin{array}{lllll}27.7 & 26.9 & 1817.9 & 1787.4 \\ 26.4 & 26.4 & 1844.3 & 1813.8\end{array}$ $\begin{array}{llll}25.6 & 24.4 & 1869.9 & 1838 .\end{array}$ \begin{tabular}{ll|llll}
28.3 & 24.6 & 1898.2 & 1862 \\
29.2 & 25.0 & 1927.4 & 1887 \\
&
\end{tabular} $31.025 .01958 .4 \quad 1912$ 33.926 .42024 .6596$. $\begin{array}{llll}35.6 & 27.0 & 3058.2 & 1993 . \\ 27.4 & 2093.8 & 2020 \\ 35 & \end{array}$

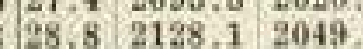
\begin{tabular}{|l|l|l|l|}
35.7 & 29.7 & 2163.8 & 2079.9 \\
27.8 & 27.8 & 2191.5 & 2107.0 \\
\hline
\end{tabular} \begin{tabular}{l|lll|l}
27.8 & 27.8 & 2191.5 & 2107.0 \\
199.0 & 27.4 & 2220.5 & 2134.4 \\
31.3 & 3.4 & 205.5 & 2161.2
\end{tabular}

34.828 .926 .62199

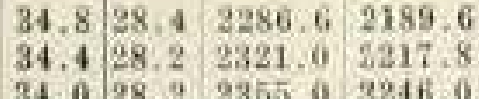

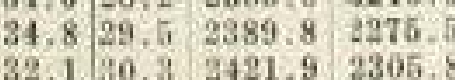
\begin{tabular}{lllll}
32.1 & 30.3 & 2421.9 & 2305.8 \\
28.7 & 28.2 & 2450.6 & 2334.0 \\
\hline
\end{tabular}

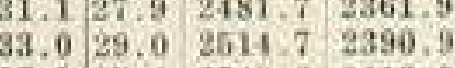
23.4 23.42560 .02441$.

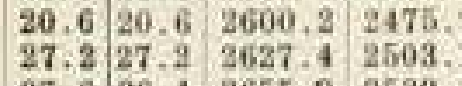
33.1 28.92688 .12658

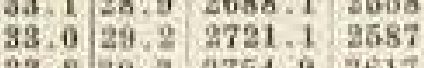

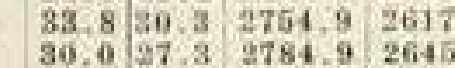
(4)

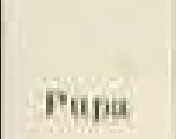

$\begin{array}{llll}2 & 7.2 & 95.9 & 05.8\end{array}$ $\begin{array}{llll}1.1 & 103.2 & 103.2 \\ 1.1 & 104.3 & 104.3\end{array}$ $\begin{array}{lllll}1.6 & 1.6 & 105.9 & 105.9 \\ 1.8 & 1.8 & 107.7 & 107 \\ 1 & .8 & 107\end{array}$

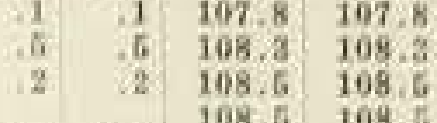

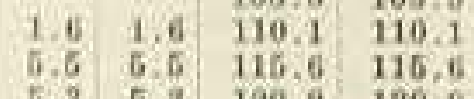
a 5121.4121 $\begin{array}{lllll}1.1 & 1.1 & 122.5 & 122.6 \\ 8.3 & 3.3 & 125.8 & 125.8 \\ 3.3 & 3.8 & 128.8 & 120.8\end{array}$ $\begin{array}{llll}2.8 & 2.8 & 128.6 & 128.6 \\ 11.9 & 11.9 & 140.6 & 140.6\end{array}$

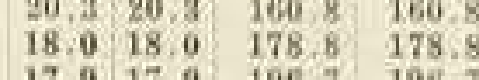
$\begin{array}{rrrrrr}17.9 & 17.9 & 196.7 & 196.7 \\ 6.1 & 6.1 & 902.8 & 202.8\end{array}$ 18.1 18.1 231. \begin{tabular}{rr|r|r|}
15.1 & 15.1 & 246.4 & 246.4 \\
9.7 & 9.7 & 256.1 & 256.1 \\
\hline & 3.7 & &
\end{tabular} $\begin{array}{rrrr}3.7 & 3.7 & 259.8 & 259.8 \\ 1.7 & 1 . & 265 & 0.8\end{array}$

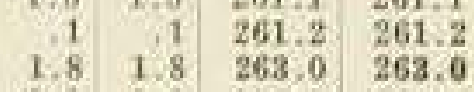
\begin{tabular}{lllll}
3.0 & 3.0 & 266.3 & 266.3 \\
\hline & 4.6 & 269.3 & 2.3
\end{tabular} \begin{tabular}{|l|l|l|l}
4.6 & 4.6 & 273.9 & 273.9 \\
1.5 & 1.5 & 275.4 & 275.4
\end{tabular}

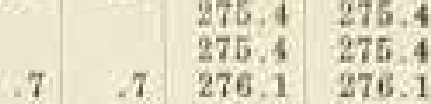

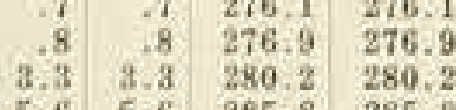
\begin{tabular}{l|l|l|l}
5.6 & 5.6 & 285.8 & 285.8 \\
6.6 & 6.6 & 292.4 & 292.4 \\
\hline & 5.3 & 297.4 & $29 .$.
\end{tabular} $\begin{array}{llll}4.3 & 4.3 & 302.0 & 302\end{array}$

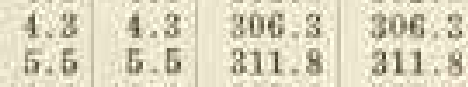
$\begin{array}{llll}4.3 & 14.3 \quad 337.2 \quad 337.2\end{array}$ \begin{tabular}{l|l|l|l|l} 
Adult & 17.6 & 17.6 & 354.8 & 354.8 \\
& 19.3 & 19.3 & 374.1 & 374.1
\end{tabular}

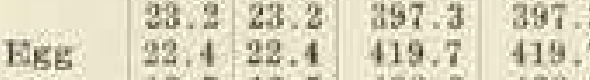
\begin{tabular}{l|l|l|l}
18.5 & 18.6 & 438.2 & 438.2 \\
12.6 & 12.6 & 450.8 & 450.8 \\
\hline
\end{tabular} $\begin{array}{llllll}3.6 & 2.6 & 454.4 & 45 . \\ 9.0 & 9.0 & 463,4 & 463.4\end{array}$ $\begin{array}{lllll}14.4 & 14.4 & 488.4 & 488 .\end{array}$ $\begin{array}{llll}17.8 & 17.8 & 506.2 & 506.2\end{array}$ 11.3
11.3
15.0

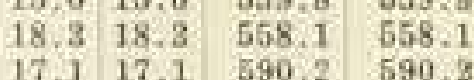
\begin{tabular}{|ll|l|l|}
\hline 17.1 & 17.1 & 590.2 & 590.2 \\
15.0 & 15.0 & 673.1 & 573.1
\end{tabular}

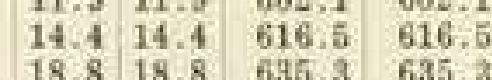
\begin{tabular}{rrrr|r}
24.8 & 24.8 & 660.1 & 660.1 \\
19.3 & 19.3 & 679.4 & 679.4
\end{tabular} \begin{tabular}{lllll}
\hline 3.3 & 23.3 & 702.7 & 702.
\end{tabular} \begin{tabular}{|l|l|l|l}
19.6 & 19.6 & 722.3 & 722. \\
\hline 8.2 & 18.2 & 740.5 & 740.
\end{tabular} \begin{tabular}{l|l|l|l}
22.2 & 23.2 & 783.8 & 783.
\end{tabular} \begin{tabular}{r|r|r|r}
\hline 24.7 & 24.7 & 808.5 & 808.5 \\
11.9 & 11.9 & 820.4 & 820.4 \\
3.6 & 3.5 & 824.0 & 824.8
\end{tabular} \begin{tabular}{r|r|r|r|}
5.2 & 5.2 & 829.2 & 829.2 \\
10.2 & 10.2 & 839.4 & 839.4
\end{tabular} $\begin{array}{llll}16.8 & 16.8 & 856.2 & 856 .\end{array}$ $\begin{array}{lllll}41.9 & 21.9 & 878.1 & 878.1\end{array}$ \begin{tabular}{rl|l|l}
22.8 & 22.8 & 920.1 & 920.1 \\
23.8 & 23.8 & 943.9 & 943.9
\end{tabular} Mature
larvi \begin{tabular}{l|l|l|l}
28.2 & 28.2 & 972.1 & 972.1 \\
\hline 27 & &
\end{tabular}

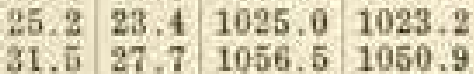
$\begin{array}{lllll}28.4 & 27.9 & 1084.9 & 1078.8\end{array}$ $\begin{array}{llll}23.2 & 23.2 & 1136.5 & 1130.4 \\ 22.6 & 22.6 & 1159.5 & 1153.0\end{array}$ $\begin{array}{llll}24.6 & 24.6 & 1183.7 & 1177 .\end{array}$ $\begin{array}{lllll}18.5 & 18.5 & 1229.0 & 1220.8\end{array}$ $\begin{array}{lllll}16.4 & 16.4 & 1245.4 & 1237.2 \\ 20.5 & 20.5 & 1265.9 & 1257.7\end{array}$ $22.6 \quad 22.6 \quad 1288.5 \quad 1280.3$ $\begin{array}{llll}28.8 & 26.3 & 1317.3 & 1306.6 \\ 29.6 & 27.3 & 1346.8 & 1333 .\end{array}$ $\begin{array}{llllll}25.7 & 24.5 & 1372.5 & 1358.4\end{array}$ $\begin{array}{llll}21.3 & 20.8 & 1417.5 & 1402\end{array}$ $\begin{array}{lllll}24.7 & 24.7 & 1442.2 & 1427.6 \\ 21.6 & 20.4 & 1463.8 & 1448.0\end{array}$ $\begin{array}{llll}4.2 & 24.2 & 1488.9 & 1472 .\end{array}$ $\begin{array}{lllll}18.4 & 18.4 & 1628.9 & 1513 .\end{array}$ $\begin{array}{lllll}42.2 & 21.8 & 1570.1 & 1553.9\end{array}$ $\begin{array}{lllll}25.7 & 25.4 & 1595.8 & 1579.8 \\ 26.0 & 25.9 & 1621.8 & 1605.2\end{array}$ \begin{tabular}{lllll}
0.2 & 25.8 & 1602.0 & 1651. \\
\hline 8.0 & 27.6 & 16800 & 1658.
\end{tabular} $\begin{array}{lllll}30.0 & 28.4 & 1710.0 & 1687.0\end{array}$ 27.425 .4 1765. 1738 $\begin{array}{llll}9.1 & 27.8 & 1829.2 & 1794 .\end{array}$ $\begin{array}{llll}3.0 & 27.8 & 1887.4 & 1850 .\end{array}$ $\begin{array}{lllll}36.6 & 39.3 & 1958.0 & 1905 .\end{array}$ $\begin{array}{lllll}85.0 & 28.7 & 1993.0 & 1934.2 \\ 29.7 & 27.7 & 2022.7 & 1961.9 \\ 2029.7 & & \end{array}$ Mature 29.3 $24.6 \quad 2078.6$ 2009.8

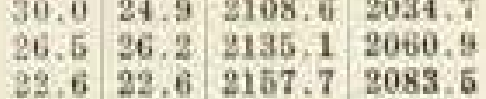
22.832 .82180 .52106$. \begin{tabular}{r|rrrrr}
16.2 & 16.2 & 2215.1 & 2140. \\
16.0 & 16.5 & 2231.6 & 2157 \\
19 & &
\end{tabular} $\begin{array}{llll}19.3 & 19.2 & 2250.8 & 3176 .\end{array}$ 21.0 21.02292 .7 2218. $\begin{array}{lllll}94.1 & 24.1 & 2316.8 & 2242 . \\ 24.8 & 24.8 & 2341.6 & 2267 .\end{array}$

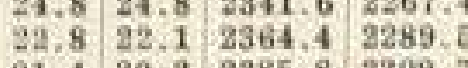
$\begin{array}{lllll}21.4 & 20.2 & 2385.8 & 2309.7 \\ 20.8 & 20.8 & 2406.6 & 3390.6 \\ 24.1 & 94.1 & 2430.7 & 2354.4 \\ 24 & \end{array}$ 
TABLE 40-Dates of the Appearance of the First Individuals of the Broods OF THE CODLING-MOTH AT OZARK, OLNEY, AND SPRINGFIELD IN 1917

\begin{tabular}{|c|c|c|c|c|c|}
\hline Generation & Stage & Ozark & Olney & $\begin{array}{r}\text { Sprin } \\
\text { field }\end{array}$ & \\
\hline Hibernating & Adults & May 12 & May 17 & May & 17 \\
\hline First & $\begin{array}{l}\text { Eggs } \\
\text { Larvæ } \\
\text { Mature larvæ } \\
\text { Adults }\end{array}$ & $\begin{array}{lr}\text { May } & 19 \\
\text { May } & 27 \\
\text { June } & 18 \\
\text { July } & 1\end{array}$ & $\begin{array}{lr}\text { May } & 20 \\
\text { June } & 1 \\
\text { June } & 23 \\
\text { July } & 7\end{array}$ & $\begin{array}{l}\text { June } \\
\text { July }\end{array}$ & $\begin{array}{r}25 \\
9\end{array}$ \\
\hline Second & $\begin{array}{l}\text { Eggs } \\
\text { Larvæ }\end{array}$ & & $\begin{array}{ll}\text { July } & 11 \\
\text { July } & 17\end{array}$ & & \\
\hline
\end{tabular}

To show the relation between the time of the appearance of the first individuals of each of the broods throughout the season at Olney Table 41 has been prepared.

The same data were worked out for Ozark in 1915, Springfield in 1916 and 1918, and Siloam Springs, Arkansas, for 1908, and the results are summarized in Table 42.

From the averages in the last column of Table 42 we find, by a little calculation, that the average accumulation of effective day-degrees from the time of the appearance of the first individuals of the first brood of pupæ, adults, eggs, and larvæ to the time of appearance of the first individuals of the second brood of the same is 979, and that the average accumulation between the time of appearance of the first individuals of the second brood of pupæ, adults, eggs, and larvæ and the time of appearance of the first individuals of the third brood of the same is 1082, the average accumulation for the complete life cycle, that is, for the period between the time of the appearance of the first eggs, larvæ, pupæ, or adults of one generation and the first eggs, larvæ, pupæ, or adults of the next generation, being 1030 .

From studies on the relation of temperature to the development of the different stages and to the whole development period in the life history, we know that there is practically no difference between the number of effective day-degrees which accumulate during these periods in the different generations. The differences shown by the data in the table are therefore undoubtedly due to the comparatively small number of observations on which the averages were based and to the fact that some of the observations were made in connection with breeding experiments in which comparatively small numbers of individuals were used. The dates on which the first larvæ of the first generation entered the fruit were determined by observations on individuals reared from hibernating larvæ, and since the number of individuals thus used was very small as compared with the whole number in the orchard, no doubt larvæ began to enter the fruit in the orchard before the first larvæ were secured in the experiments. The dates when the first larvæ of the second genera- 
TABLE 42-Accumulated Effective Day-degree at the Time of the Observed Appearance of the First Individuals of each Brood

\begin{tabular}{|c|c|c|c|c|c|c|c|c|c|}
\hline \multirow{2}{*}{ Generation } & \multirow{2}{*}{ Stage } & \multicolumn{3}{|c|}{ Olney } & Ozark & \multicolumn{2}{|c|}{ Springfield } & \multirow{2}{*}{$\frac{\begin{array}{c}\text { Siloam } \\
\text { Springs, } \\
\text { Ark.* }\end{array}}{1908}$} & \multirow[t]{2}{*}{ Average } \\
\hline & & 1915 & 1916 & 1917 & 1915 & 1916 & 1918 & & \\
\hline Hibernating & $\begin{array}{l}\text { Pupa } \\
\text { Adult }\end{array}$ & 315 & $\begin{array}{l}115 \\
356\end{array}$ & $\begin{array}{l}104 \\
355\end{array}$ & 364 & $\begin{array}{l}108 \\
313\end{array}$ & $\begin{array}{l}110 \\
345\end{array}$ & 324 & $\begin{array}{r}109 \\
339\end{array}$ \\
\hline First & $\begin{array}{l}\text { Egg } \\
\text { Larva } \\
\text { Mature larva } \\
\text { Pupa } \\
\text { Adult }\end{array}$ & $\begin{array}{r}422 \\
585 \\
968 \\
1075 \\
1309\end{array}$ & $\begin{array}{r}415 \\
581 \\
967 \\
1034 \\
1337\end{array}$ & $\begin{array}{r}420 \\
573 \\
972 \\
1079 \\
1307\end{array}$ & $\begin{array}{r}534 \\
860 \\
1248\end{array}$ & $\begin{array}{r}400 \\
574 \\
1082 \\
1169 \\
1399\end{array}$ & $\begin{array}{l}1066 \\
1272\end{array}$ & $\begin{array}{r}371 \\
623 \\
1024 \\
1285\end{array}$ & $\begin{array}{r}406 \\
578 \\
970 \\
1074 \\
1308\end{array}$ \\
\hline Second & $\begin{array}{l}\text { Egg } \\
\text { Larva } \\
\text { Mature larva } \\
\text { Pupa } \\
\text { Adult }\end{array}$ & $\begin{array}{l}1415 \\
1557 \\
2079 \\
2168 \\
2429\end{array}$ & $\begin{array}{l}1410 \\
1566 \\
2079 \\
2161 \\
2418\end{array}$ & $\begin{array}{l}1403 \\
1532 \\
2009 \dagger \\
2106 \dagger \\
2377 \dagger\end{array}$ & $\begin{array}{l}1518 \\
1999 \\
2100 \\
2321\end{array}$ & $\begin{array}{l}1454 \\
1602 \\
2134 \\
2246 \\
2430\end{array}$ & 2302 & $\begin{array}{l}1389 \\
1528 \\
2034 \\
\\
2423\end{array}$ & $\begin{array}{l}1414 \\
1551 \\
2065 \\
2169 \\
2387\end{array}$ \\
\hline Third & $\begin{array}{l}\text { Egg } \\
\text { Larva }\end{array}$ & $\begin{array}{l}2471 \\
2645\end{array}$ & $\begin{array}{l}2455 \\
2618\end{array}$ & $\begin{array}{l}2424 \dagger \\
2576 \dagger\end{array}$ & 2568 & $\begin{array}{l}2490 \\
2688\end{array}$ & & $\begin{array}{l}2477 \\
2643\end{array}$ & $\begin{array}{l}2471 \\
2632\end{array}$ \\
\hline
\end{tabular}

* Data in this column were obtained by the use of the 1908 records of the U. S. Weather Bureau for Fayetteville, Arkansas, and the seasonal history records of the codling-moth as given by E. L. Jenne in Bulletin No. 80, Part I, of the Bureau of Entomology,

$\dagger$ Numbers followed by a dagger were computed. 
tion entered the fruit was determined from observations on individuals secured from band collections. These were abundant enough to give ts the dates with a fair degree of accuracy. The number of effective daydegrees, 1551 , or, to use round numbers, 1550, given for this event in the table may be considered as approximately correct and will be used as a basis for making corrections in the table. The dates when the first larvæ of the third generation entered the fruit were determined from a comparatively small number of individuals isolated for the purpose of life history studies, and for this reason it is quite certain that the dates thus obtained were somewhat later than the true dates.

It is impossible to estimate with any degree of accuracy how much of an error was made in determining dates from a too limited amount of material, but we can, by using data procured by studies on the relation of temperature to the different stages and to the complete development period, determine the approximate minimum effective day-degrees which accumulate during the complete life cycle; and by using 1550 as the total effective day-degrees at the time when the first larvæ of the second generation appear we can determine the total effective day-degrees for the date on which the first egg, larvæ, pupæ, and adults of the three generations may be expected to appear.

From our studies on the relation of temperature to the development of the three stages we concluded (see page 280) that the minimum accumulation for the three stages was about 939, the average 1101, and the maximum about 1317; and from our studies on the life history of 348 individuals we concluded that the minimum accumulation for the period of development was about 950 , the average 1120 , and the maximum about 1350 (see page 259). To get the total accumulation for the complete life cycle, it will be necessary to add to these mimima, averages, and maxima about 50,75 , and 250 , respectively, because the deposition of the first eggs, the time of maximum oviposition, and the laying -of the last eggs do not occur until about the first, the third, and the tenth day, respectively, after the emergence of the female. (Table 43.)

None of the minima, 989,1000 , and 1030 , obtained for the complete life cycle by the different methods was determined with absolute accuracy, but they were based on actual observations, and the fact that they do not differ very widely indicates that they are approximately correct. For practical purposes either one might be used as the minimum, but the second minimum, 1000 , being nearly the average of the three is perhaps more nearly correct than the others, and it will be used as the basis for the discussion which follows.

By dividing the 1000 effective day-degrees into parts proportional to the minimum effective day-degrees required for the egg, larval, and pupal periods, making allowance for the period elapsing between the time of the emergence of the adult female and the deposition of the first eggs, and by taking 1550 (Table 42) as approximately the correct accumulation at the time when the larvæ of the second generation begin 
to enter the fruit, we can construct a consistent table showing the approximate accumulations which indicate the time when the first pupæ, adults, eggs, and larvæ of the three generations may be expected to appear. Table 44, however, for the present, must be regarded as only a somewhat close approximation to the correct accumulations. It may be necessary to make some changes as a result of further observations.

TABLE 43-Accumulation of Effective DaY-degrees during Stages, duRing Entire Development Period, and during the Complete Life Cycle

\begin{tabular}{|c|c|c|c|c|}
\hline & \multirow{2}{*}{ Period } & \multicolumn{3}{|c|}{ Accumulation } \\
\hline & & Minimum & Average & Maximum \\
\hline \multirow{3}{*}{ Stages } & $\begin{array}{l}\text { Egg } \\
\text { Larva } \\
\text { Pupa }\end{array}$ & $\begin{array}{l}156 \\
527 \\
256\end{array}$ & $\begin{array}{l}163 \\
673 \\
265\end{array}$ & $\begin{array}{l}170 \\
873 \\
274\end{array}$ \\
\hline & Adult to egg & $\begin{array}{r}939 \\
50\end{array}$ & $\begin{array}{r}1101 \\
75\end{array}$ & $\begin{array}{r}1317 \\
250\end{array}$ \\
\hline & Life cycle & 989 & 1176 & 1567 \\
\hline \multirow{2}{*}{ Life history } & $\begin{array}{l}\text { Development period } \\
\text { Adult to egg }\end{array}$ & $\begin{array}{r}950 \\
50\end{array}$ & $\begin{array}{r}1120 \\
75\end{array}$ & $\begin{array}{r}1350 \\
250\end{array}$ \\
\hline & Life cycle & 1000 & 1195 & 1600 \\
\hline Seasonal history & Life cycle & 1030 & & \\
\hline
\end{tabular}

TABLe 44-Accumulated Effective Day-degrees which Indicate the Trme when the First Eggs, Larvae, Pupae, and Adults may be EXPECTED TO APPEAR

\begin{tabular}{|c|c|c|}
\hline Generation & Stage & Day-degrees \\
\hline Hibernating & $\begin{array}{l}\text { First pupæ } \\
\text { First adults }\end{array}$ & $\begin{array}{r}82 \\
340\end{array}$ \\
\hline First generation & $\begin{array}{l}\text { First eggs } \\
\text { First larvæ } \\
\text { First pupæ } \\
\text { First adults }\end{array}$ & $\begin{array}{r}392 \\
550 \\
1082 \\
1342\end{array}$ \\
\hline Second generation & $\begin{array}{l}\text { First eggs } \\
\text { First larvæ } \\
\text { First pupæ } \\
\text { First adults }\end{array}$ & $\begin{array}{l}1392 \\
1550 \\
2082 \\
2342\end{array}$ \\
\hline Third generation & $\begin{array}{l}\text { First eggs } \\
\text { First larvæ }\end{array}$ & $\begin{array}{l}2392 \\
2550\end{array}$ \\
\hline
\end{tabular}


table 45-Average Normal Daty Effeotive Day-degrees, Acoumurations of the Same, and Dates whFi the First Individuals of the Different Stages of eagh Generation aray be expeotfi to appear at Olney in a Nobmal Season

\begin{tabular}{|c|c|c|c|c|c|c|c|c|c|c|c|c|c|c|c|c|c|c|}
\hline \multirow[b]{2}{*}{$\stackrel{g}{g}$} & \multicolumn{3}{|c|}{ March. } & \multicolumn{3}{|c|}{ April. } & \multicolumn{3}{|c|}{ May. } & \multicolumn{3}{|c|}{ June. } & \multicolumn{3}{|c|}{ July. } & \multicolumn{3}{|c|}{ A ugust. } \\
\hline & 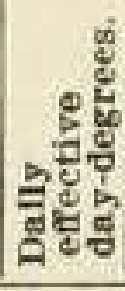 & 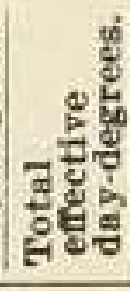 & 离 & 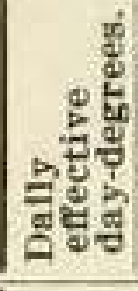 & 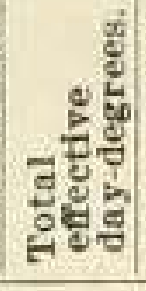 & 竞 & 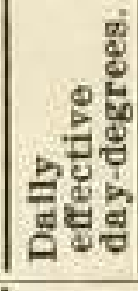 & 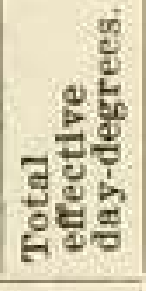 & 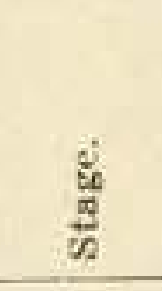 & 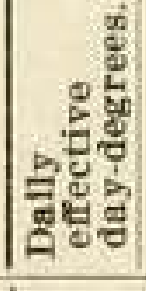 & 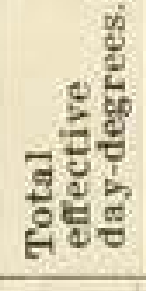 & $\frac{\text { of }}{5}$ & 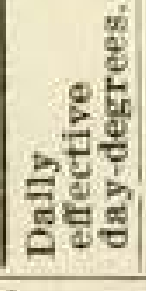 & 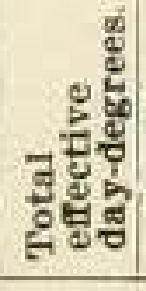 & क्ष & 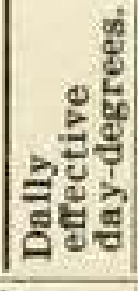 & 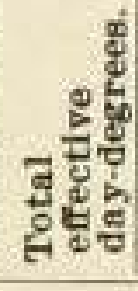 & $\sum_{0}^{0}$ \\
\hline 1 & 1 & 1 & & 5 & 91 & & 11 & 296 & & 20 & 789 & & 25 & 1465 & & 26 & 2264 & \\
\hline 2 & 1 & 2 & & 5 & 96 & & 11 & 307 & & 20 & 809 & & 25 & 1490 & & 26 & 2290 & \\
\hline 3 & 1 & 3 & & 5 & 101 & & 12 & 319 & & 20 & 829 & & 25 & 1515 & & 26 & 2316 & \\
\hline 4 & 1 & 4 & & 5 & 106 & & 12 & 331 & & 20 & 849 & & 25 & 1540 & Larva & 26 & 2342 & Adult \\
\hline 5 & 1 & 5 & & 5 & 111 & & 13 & 344 & Aduit & 20 & 869 & & 25 & 1565 & & 26 & 2368 & \\
\hline i & 1 & 6 & & 5 & 116 & & 13 & 357 & & 20 & 899 & & 25 & 1590 & & 25 & 2393 & Egg \\
\hline 7 & 1 & 7 & & 5 & 121 & & 13 & 370 & & 20 & 909 & & 26 & 1616 & & 25 & 2418 & \\
\hline 8 & 2 & 9 & & 5 & 126 & & 14 & 384 & & 20 & 929 & & 26 & 1642 & & 25 & 2443 & \\
\hline 9 & 2 & 11 & & 5 & 131 & & 14 & 398 & Egg & 21 & 950 & & 26 & 1668 & & 25 & 2468 & \\
\hline 10 & 2 & 13 & & 5 & 136 & & 14 & 412 & & 21 & 971 & & 26 & 1694 & & 25 & 2493 & \\
\hline 11 & 2 & 15 & & 5 & 141 & & 14 & 426 & & 21 & 992 & & 26 & 1720 & & 25 & 2518 & \\
\hline 12 & 2 & 17 & & 5 & 146 & & 15 & 441 & & 21 & 1013 & & 26 & 1746 & & 25 & 2543 & Larva \\
\hline 13 & 3 & 20 & & 6 & 152 & & 15 & 456 & & 22 & 1035 & & 27 & 1773 & & 25 & 2568 & \\
\hline 14 & 3 & 23 & & 6 & 158 & & 15 & 471 & & 22 & 1057 & & 27 & 1800 & & 25 & 2593 & \\
\hline 15 & 3 & 26 & & 6 & 164 & & 15 & 486 & & 22 & 1079 & Pupa & 27 & 1827 & & 26 & 2619 & \\
\hline 16 & 3 & 29 & & 6 & 170 & & 15 & 501 & & 22 & 1101 & & 27 & 1854 & & 26 & 2645 & \\
\hline 17 & 3 & 32 & & 6 & 176 & & 16 & 517 & & 22 & 1123 & & 27 & 1881 & & 26 & 2671 & \\
\hline 18 & 3 & 35 & & 7 & 183 & & 16 & 533 & & 23 & 1146 & & 27 & 1908 & & 26 & 2697 & \\
\hline 19 & 3 & 38 & & 7 & 190 & & 16 & 549 & Larva & 23 & 1169 & & 27 & 1935 & & 26 & 2723 & \\
\hline 20 & 4 & 42 & & 7 & 197 & & 17 & 566 & & 23 & 1192 & & 26 & 1961 & & 25 & 2748 & \\
\hline 21 & 4 & 46 & & 7 & 204 & & 17 & 583 & & 24 & 1216 & & 26 & 1987 & & 24 & 2772 & \\
\hline 22 & 4 & 50 & & 8 & 212 & & 17 & 600 & & 24 & 1240 & & 26 & 2013 & & 24 & 2796 & \\
\hline 23 & 4 & 54 & & 8 & 220 & & 18 & 618 & & 25 & 1265 & & 25 & 2038 & & 24 & 2720 & \\
\hline 24 & 4 & 58 & & 9 & 229 & & 18 & 636 & & 25 & 1290 & & 25 & 2063 & & 24 & 2844 & \\
\hline 25 & 4 & 62 & & 9 & 238 & & 18 & 654 & & 25 & 1315 & & 25 & 2088 & Pupa & 24 & 2868 & \\
\hline 26 & 4 & 66 & & 9 & 247 & & 18 & 672 & & 25 & 1340 & Adult & 25 & 2113 & & 23 & 2891 & \\
\hline 27 & 4 & 70 & & 9 & 256 & & 19 & 691 & & 25 & 1365 & & 25 & 2138 & & 22 & 2913 & \\
\hline 28 & 4 & 74 & & 9 & 265 & & 19 & 710 & & 25 & 1390 & Egg & 25 & 2163 & & 22 & 2935 & \\
\hline 29 & 4 & 78 & & 10 & 275 & & 19 & 729 & & 25 & 1415 & & 25 & 2188 & & 22 & 2957 & \\
\hline 30 & 4 & 82 & Pupa & 10 & 285 & & 20 & 749 & & 25 & 1440 & & 25 & 2213 & & 22 & 2979 & \\
\hline 31 & 4 & 86 & & & & & 20 & 769 & & & & & 25 & 2238 & & 22 & 3001 & \\
\hline
\end{tabular}




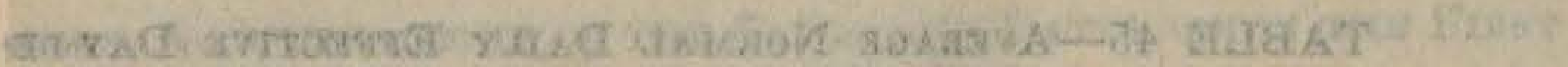

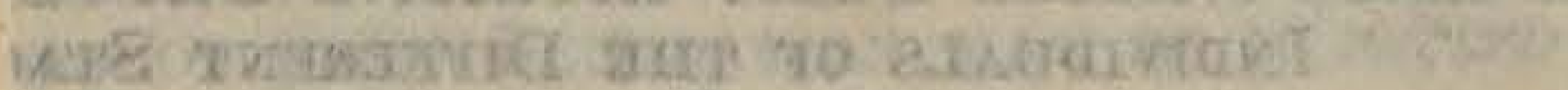

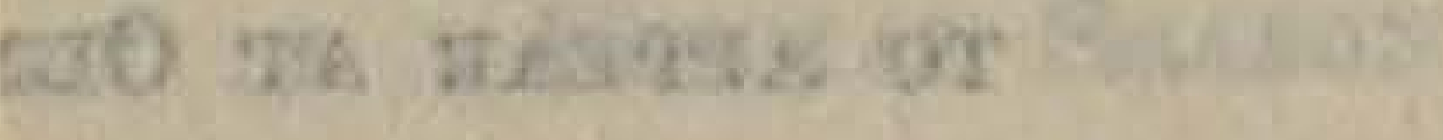

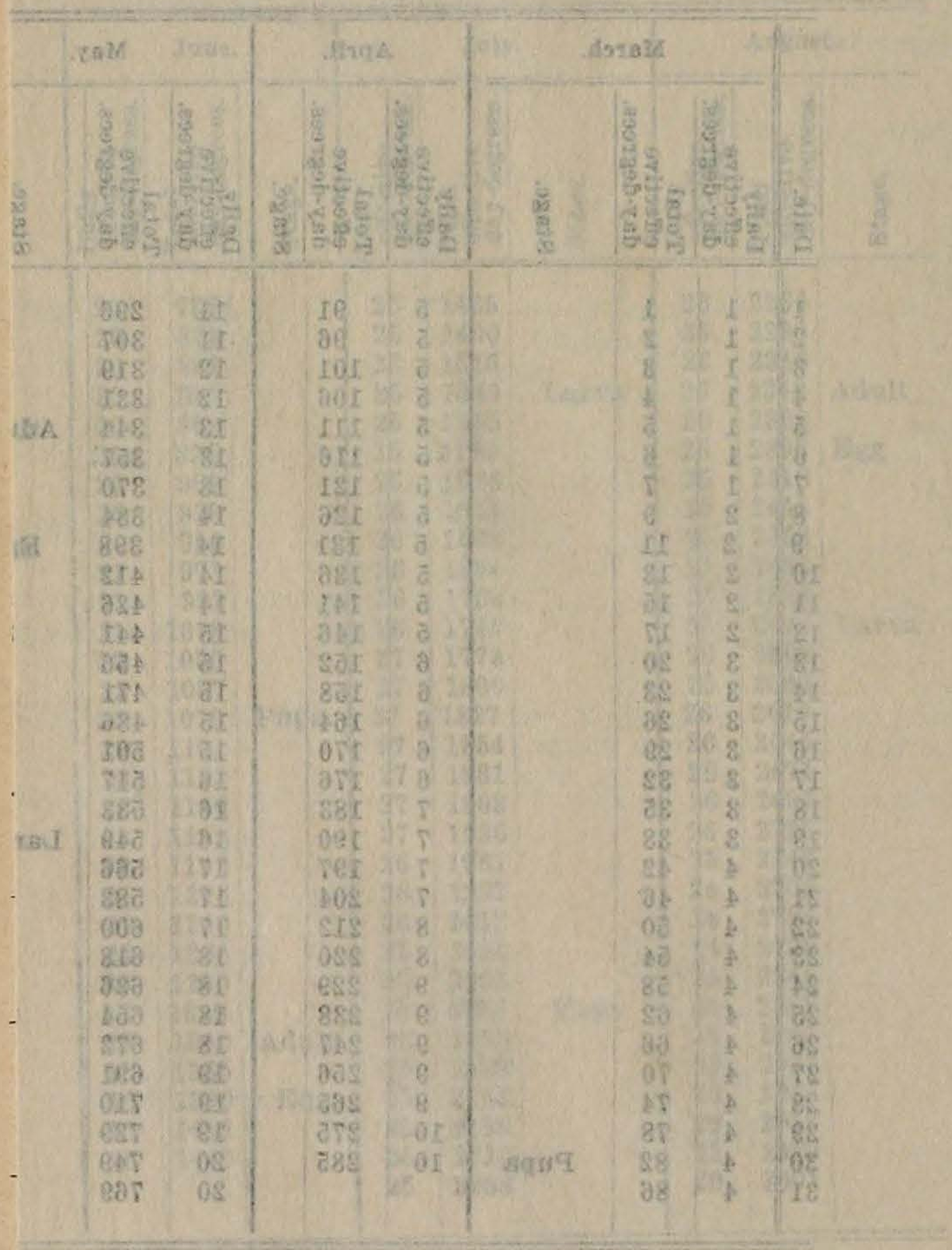




\section{Method of Forecasting Dates when the Different Stages OF THE SEVERAL GENERATIONS MAY BE EXPECTED TO APPEAR at any given Place in a Normal Season}

By ascertaining the normal daily effective day-degrees at any point in the state throughout the season and ascertaining the total sums of the daily accumulations for each day of the season and comparing the sums on the different dates with Table 44, we can ascertain the approximate dates when the different stages of each generation may be expected to appear. Table 45 gives the normal daily effective day-degrees, the total effective day-degrees, and the dates on which the first individuals of the stages of each generation normally appear at Olney, Illinois.

This table was computed from the maximum and minimum readings of the U. S. Weather Bureau at Olney from 1901 to 1916 inclusive by the formulæ given on page 283 . In all of these computations the daily effective day-degrees were taken as the average degrees above $50^{\circ}$ F. diminished by twice the average degrees above 86 .

It is not to be expected that the first eggs, larvæ, pupæ, and adults will appear in any locality on the exact dates shown in a table of the normal accumulations of effective day-degrees for that locality. Such tables will give only the approximate dates. There are many factors which may influence the time of appearance: there are always periods during every season when the temperatures are not normal; there are situations in every locality in which the temperatures differ from those of the place where the recording instrument is located, such as steep northern and steep southern slopes, and differences in altitudes in hilly countries; and pupæ on the south side of the trunks of trees will be subjected to greater heat than those on the north side.

There is no practical method by which all the local factors may be taken into account, but the seasonal variations may be anticipated by keeping a daily record of the total accumulation of effective day-degrees in each of the fruit-growing sections; and dates when the first larvæ of each generation may be expected to enter the fruit may be anticipated however abnormal the weather may be. When the accumulations amount to 390,1290 , and 2280 the first larvæ of the first, second, and third generations, respectively, may be expected to enter the fruit in about 10 days. (See Table 51, p. 289.)

Table 43 (p. 280) shows that the average accumulation of effective day-degrees for the complete life cycle, as ascertained by a study of the different stages, is 1176 , the maximum being about 1567 , and that by a study of the life history the average accumulation is 1195 , the maximum being about 1600 . These differences arise partly from the fact that in the former case 86,85 , and 87 degrees $F$. were used as the degrees of maximum rate of development of the egg, larva, and pupa, respectively, whereas in the latter case 86 was used for each of the stages. Since it is much more convenient to use 86,1195 should be used as the approximate average. 
It follows that since the first and the second generations are almost complete in the southern half of Illinois, if the larvæ of the first generation are observed to be entering the fruit in large numbers on a certain date, larvæ of the second generation will be entering the fruit in large numbers after an interval during which the accumulated effective daydegrees have reached 1195, unless delaying factors, such as parasites, rainy weather at hatching time, or other cause which might occasion great mortality of eggs, larvæ, or pupæ, should intervene. This would not hold true for the third generation, since it is not complete, for, at best, only a small proportion of the larvæ of the second generation transform.

The maximum number of effective day-degrees which may accumulate between the deposition of an egg of one generation and the deposition of an egg of the next generation is about 1600 . If a number of eggs are deposited on the same day the deposition of eggs by females developed from them will be extended over a period during which the accumulation of effective day-degrees ranges from 1000 to 1600 , which corresponds to a period of about 20 days. This accounts largely for the overlapping of the second and third generations notwithstanding the fact that the first and second do not overlap.

\section{How to compute Effective Day-Degrees}

One of the main objects of this investigation was to find, if possible. a single factor in terms of which the progress of the seasonal history of the insect could be followed and even predicted long enough beforehand to be of practical value. Apparently we have found that factor in temperature expressed in terms of effective day-degrees as the unit. Humidity, evaporation, and rainfall may affect the rate of development to some extent, but in the case of the codling-moth these factors have such a small influence under out-of-door conditions that for practical purposes they may be disregarded, leaving temperature as the single factor to be taken into account.

The temperatures that are to be taken into account are the temperatures above 50 degrees and those above 86 . To ascertain the number of effective day-degrees that have accumulated during any given day, first find the average degrees above 50 degrees $\mathrm{F}$., then the average degrees above 86 degrees, multiply the latter by two and subtract the product from the former. The result will be the effective day-degrees.

To compute the effective day-degrees, accurate thermograph records should be kept. The average daily temperatures above 50 degrees $F$. can be computed by adding together the hourly readings above 50 and dividing the sum by 24 , and the average daily temperatures above 86 can be computed in the same manner. If the drum type of thermograph be used the temperature curve above 50 and above 86 may be integrated by means of a planimeter, expressing the result as hourly-degree units, then by dividing the totals for each day by 24 , the average hourly-degrces above 50 and 86 may be ascertained, which are the day-degrees in each case. Subtracting from the day-degrees above 50 twice the day-degrees above 86 gives the number of effective day-degrees. 
In case thermograph records are not available the effective daydegrees can be determined approximately from maximum and minimum readings by the use of the following formulæ proposed by Dr. S. A. Forbes.

Formulae for computing the average day-degrees above any given temperature, using the maximum temperature, and the minimum temperatures preceding and following it.

$a=$ Number of degrees that the first minimum is above or below the given temperature.

$b=$ Number of degrees that the second minimum is above or below the given temperature.

$c=$ Number of degrees that the maximum is above the given temperature.

$x=$ Number of day-degrees above the given temperature.

I. When both minima are at or above the given temperature,

$$
x=\frac{a+b+2 c}{4} .
$$

II. When the first minimum is below the given temperature,

$$
x=\frac{b+c}{4}+\frac{c^{2}}{4(a+c)} .
$$

III. When the second minimum is below the given temperature,

$$
x=\frac{a+c}{4}+\frac{c^{2}}{4(b+c)} .
$$

IV. When both minima are below the given temperature,

$$
x=\frac{c^{2}}{4(a+c)}+\frac{c^{2}}{4(b+c)} .
$$

When the thermograph is used it should be kept correct by checking it up daily with the maximum and minimum thermometers, and the recording instrument should be kept in a place which has the same conditions as to temperature as the orchards to which the records are to be applied. It is very evident that the maximum and minimum readings taken in the heart of a city would not give readings that would apply to adjacent country districts.

\section{Relation of Temperature to Time when larvae Hibernate}

In the spring, hibernating larvæ will begin to transform as soon as temperatures rise above $52^{\circ} \mathrm{F}$., but after midsummer, soon after the temperatures begin to fall, maturing larvæ cease to transform long before the temperatures fall below the 52 point. 
Generally, larvæ that mature prior to August 1 transform; those that mature after September 1, hibernate; and of those that mature during August some hibernate and some transform, the percentage of those that hibernate usually increasing as the month advances, the rate of increase depending upon the temperatures.

The time when hibernation takes place is determined largely by the habit of the species, but it may be somewhat modified by temperatures. While at Olney hibernation did not usually take place until August, a few unusually cool days caused some larvæ to hibernate as early as the first week in July in 1915, and in 1916 hibernation was delayed till the middle of August by continuous warm weather. Some larvæ hibernate any time after midsummer when the minimum daily temperatures fall to $60^{\circ}$ or below and the mean daily temperatures to about $70^{\circ}$ or below; other larvæ do not hibernate until such temperatures have prevailed for two or three weeks. During the latter half of August hibernation is induced much more readily than earlier in the month, and with rare exceptions larvæ maturing after September 1 hibernate irrespective of temperatures.

Table 46 illustrates what has just been said concerning hibernation.

Note that in 1915, 4.4 per cent. of the larvæ which matured during the period from July 4 to July 7 , at the close of a week of unusually cool weather, hibernated. Another period of cool weather ended with July 25 , and 5 per cent. of the larvæ maturing during the period from July 25 to July 28 hibernated. The next period of cool weather began August 4 , and 7 per cent. of the larvæ maturing during the period from August 4 to August 7 hibernated. From this time on, the mean daily temperatures remained near $70^{\circ}$ or below, and the percentage of hibernating larvæ increased very rapidly until August 27. All larvæ under observation maturing after this date hibernated with the exception of two. The two larvæ which transformed, matured during a period of twelve exceptionally warm days following September 7.

In 1916 there were no cool days in July and none in August until the 14th. No larvæ hibernated in July. A few that hibernated prior to August 14, hibernated apparently purely from habit. The two cool days of August 14 and 15 may have hastened hibernation, but the percentage of those that hibernated increased rapidly from the 16th to the $22 \mathrm{~d}$, although the weather was unusually warm, showing that after the middle of August larvæ, will yield to their habit regardless of temperatures. The delay in the time of hibernation in 1916, as compared with 1915, was undoubtedly due to the higher temperatures in 1916 during August.

\section{Relation of Humidity and Evaporation to the Rate of Development of the Codling-moth}

When we began the preliminary study of our accumulated data on this subject they indicated clearly that temperature was the controlling factor in determining the rate of development of the stages of the codling-moth, and that humidity and evaporation had at most only a very 


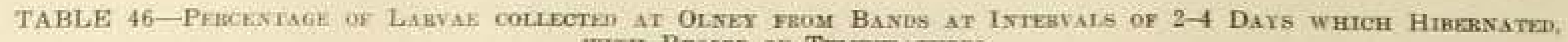
WTIH RECORD OF TRAPERATURES

\begin{tabular}{|c|c|c|c|c|c|c|c|c|c|c|c|c|c|}
\hline \multirow{2}{*}{\multicolumn{2}{|c|}{$\begin{array}{l}\text { Date of } \\
\text { collection. }\end{array}$}} & \multicolumn{6}{|c|}{1915.} & \multicolumn{6}{|c|}{1916.} \\
\hline & & & Tempers & & Per & cent: hiberna & ted. & & Tempern & & Per & t. bib & nated \\
\hline & & $\underset{90}{\operatorname{Max}}$ & Min. & Mean & Male & Female & $\mathrm{ML} \& \mathrm{~F}$. & $\operatorname{Max}$. & Min: & Mean & M. & F: & M.\& F \\
\hline " w & $\frac{1}{2}$ & $\begin{array}{l}99 \\
83\end{array}$ & $\begin{array}{l}64 \\
61\end{array}$ & $\begin{array}{l}71 \\
72\end{array}$ & $\ldots-x+\ldots$ & $\ldots+n+\cdots=$ & $x+42, \ldots$ & $\begin{array}{l}87 \\
92\end{array}$ & $\begin{array}{l}69 \\
67\end{array}$ & $\begin{array}{l}79 \\
80\end{array}$ & & & \\
\hline w & 3 & 72 & 63 & 70 & & $28250=24$ & 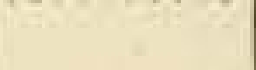 & 89 & 71 & 80 & & & \\
\hline w & 4 & 77 & 58 & $65^{\circ}$ & & & 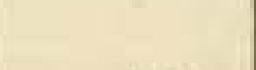 & 83 & 65 & 75 & & & \\
\hline$\ddot{*}$ & 5 & 74 & 50 & 61 & 5.8 & 3.3 & & 85 & 61 & 74 & & & \\
\hline$\ddot{*}$ & 6 & 77 & 52 & 66 & & 0.0 & 7,7 & 86 & 61 & 74 & & & \\
\hline$\ddot{u}$ & 7 & & 62 & 69 & & & & 89 & 64 & 77 & & & \\
\hline 4 & 8 & 69 & 64 & $68^{1}$ & & & & 90 & 66 & 78 & & & \\
\hline$\because 4$ & 9 & 73 & 60 & 68 & $\cdots \cdots$ & $\cdots+\infty$ & \% & 86 & 66 & 75 & & & \\
\hline $\begin{array}{ll}* & 1 \\
* & 1\end{array}$ & 10 & 84 & 67 & 72 & & & & 87 & 65 & 76 & & & \\
\hline 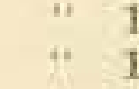 & $\begin{array}{l}11 \\
12\end{array}$ & $\begin{array}{l}86 \\
81\end{array}$ & $\begin{array}{l}65 \\
68\end{array}$ & 74 & $\ldots \ldots \ldots$ & $\therefore$ & 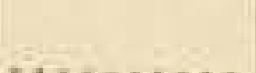 & & 68 & $\begin{array}{l}78 \\
77\end{array}$ & & & \\
\hline 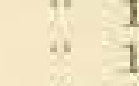 & 13. & 87 & 69 & 771 & 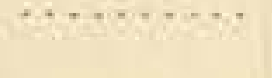 & & $\cdots+x+2$ & $\begin{array}{l}78 \\
88\end{array}$ & $\begin{array}{l}73 \\
68\end{array}$ & $\begin{array}{l}77 \\
76\end{array}$ & & & \\
\hline " & 14 & 90 & 72 & 80 & & & & 87 & 69 & 77 & & & \\
\hline$H$ & 15 & 91 & 74 & 83 & $\ldots+\ldots$ & W.2. & n. & 91 & 72 & 82 & & & \\
\hline " & 16 & 90 & 75 & 82 & & & & 94 & 71 & $\$ 2$ & & & \\
\hline 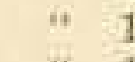 & 17 & 88 & 75 & $80^{\circ}$ & 60 & & & 94 & 70 & 81 & & & \\
\hline$\%$ & 18 & 89 & 73 & 81 & $\ldots \ldots$ & $\cdots$ & nat. & 93 & 71 & 81 & & & \\
\hline$"$ & 19 & 86 & 71 & 78 & & & & 92 & 71 & $\begin{array}{l}80 \\
78\end{array}$ & & & \\
\hline $\begin{array}{l}\text { "1. } 2 \\
\text { *. } 2\end{array}$ & $\begin{array}{l}20 \\
21\end{array}$ & $\begin{array}{l}80 \\
78\end{array}$ & $\begin{array}{l}71 \\
58\end{array}$ & $\begin{array}{l}77 \\
69\end{array}$ & $\ldots \ldots$ & & . & $\begin{array}{l}90 \\
87\end{array}$ & 70 & $\begin{array}{l}78 \\
76\end{array}$ & & & \\
\hline .) 2 & 22 & $\begin{array}{l}78 \\
79\end{array}$ & $\begin{array}{l}58 \\
58\end{array}$ & $\begin{array}{l}69 \\
69\end{array}$ & & & & $\begin{array}{l}87 \\
90\end{array}$ & & $\begin{array}{l}76 \\
76\end{array}$ & & & \\
\hline$\mu$ & 23 & 82 & 58 & $70\}$ & $\ldots \ldots$ & $+\pi$ & $\ldots \ldots \ldots$ & 94 & $\begin{array}{l}61 \\
65\end{array}$ & $\begin{array}{l}76 \\
79\end{array}$ & & & \\
\hline “ & 24 & 82 & 61 & $71\}$ & & & & 94 & 65 & 79 & & & \\
\hline 4 & 25 & 84 & 64 & 72 & & & & 96 & 67 & $81\}$ & & & \\
\hline " & 26 & 86 & 70 & 76 & $\ldots \ldots, \ldots \ldots$ & 6.7 & 5. & 97 & 68 & $82\}$ & & & \\
\hline w & 27 & 87 & 70 & 77 & 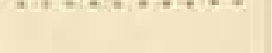 & & $0 .+$ & 99 & 70 & 84 & & & \\
\hline$*$ & 28 & 90 & 70 & 77 & & & & 97 & 70 & 84 & & & \\
\hline " & 29 & 82 & 72 & 79 & & & & 98 & 75 & 86 & & & \\
\hline 4 & 30 & 87 & 73 & 79 & $\cdots \cdots, \cdots$ & $\cdots, \cdots \cdots \cdots$ & $\ldots \ldots \ldots \ldots$ & 95 & 73 & 84 & & & \\
\hline (" 3 & 31 & 91 & 74 & 82 & & & & 99 & 76 & 86 & & & \\
\hline Aug. & 1 & 86 & 72 & 77 & & & & 83 & 71 & 77 & & & \\
\hline$\theta$ & 2 & 84 & 71 & 76 & $\cdots+\cdots \cdots$ & $\cdots \cdots$ & n....... & 91 & 70 & 79 & & & \\
\hline$\ddot{*}$ & $\begin{array}{l}3 \\
4\end{array}$ & $\begin{array}{l}80 \\
70\end{array}$ & 73 & $\begin{array}{l}76 \\
65\end{array}$ & & & & 95 & 70 & $\begin{array}{l}81 \\
84\end{array}$ & & & \\
\hline$\ddot{4}$ & $\frac{4}{5}$ & $\begin{array}{l}70 \\
75\end{array}$ & 61 & $\left.\begin{array}{l}65 \\
64\end{array}\right\}$ & 7. & 1. & 7. & 98 & 73 & $\begin{array}{l}84 \\
84\end{array}$ & & & \\
\hline 4 & 6 & $\begin{array}{l}75 \\
82\end{array}$ & $\begin{array}{l}56 \\
60\end{array}$ & $\left.\begin{array}{l}64 \\
70\end{array}\right\}$ & & & & $\begin{array}{l}97 \\
97\end{array}$ & 73 & $\begin{array}{l}84 \\
84\end{array}$ & 2 & & \\
\hline & 7 & $\begin{array}{l}82 \\
84\end{array}$ & $\begin{array}{l}60 \\
57\end{array}$ & 70 & & & & & 74 & $\begin{array}{l}84 \\
85\end{array}$ & & & 1 \\
\hline$\because$ & 8 & 85 & 68 & 76 & 11. & $\cdots \cdots$ & 5. & $\begin{array}{l}97 \\
90\end{array}$ & $\begin{array}{l}73 \\
72\end{array}$ & $\begin{array}{l}80 \\
82\end{array}$ & & & \\
\hline$\cdots$ & 9 & 81 & 65 & 75 & & & & 89 & 70 & 78 & 2 & & 1 \\
\hline ." & 0 & 72 & 71 & 72 & & & & 93 & 73 & 81 & & & \\
\hline$*$ & 1 & 71 & 67 & 68 & 39. & 23. & 27. & 96 & 72 & $83\}$ & 2 & 3 & 3 \\
\hline H & 2 & 77 & 60 & 69 & & & & 87 & 72 & 783 & 2 & 0 & a \\
\hline 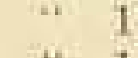 & 3 & 83 & 60 & 70 & 32. & 19. & 25. & 73 & 67 & 73 & & & \\
\hline H 1 & 4 & 74 & 67 & 71 & & ter. & so. & 71 & 56 & 64 & & 10 & is \\
\hline * 1 & 5 & 83 & 61 & $\begin{array}{l}71 \\
77\end{array} \mid$ & & & & 79 & 67 & 70 & & - 0 & \\
\hline 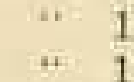 & 6 & 88 & $\begin{array}{l}70 \\
69\end{array}$ & $\left.\begin{array}{l}77 \\
74\end{array}\right\}$ & 83. & 71. & 77. & $\begin{array}{l}86 \\
90\end{array}$ & $\begin{array}{l}70 \\
66\end{array}$ & $\begin{array}{l}77 \\
77\end{array}$ & & & \\
\hline $\begin{array}{l}* 1 \\
\text { *. } 1\end{array}$ & $\begin{array}{l}7 \\
8\end{array}$ & $\begin{array}{l}82 \\
65\end{array}$ & $\begin{array}{l}69 \\
62\end{array}$ & 67 & & & & 94 & $\begin{array}{l}66 \\
74\end{array}$ & $\left.\begin{array}{l}77 \\
83\end{array}\right\}$ & 44 & 61 & 53 \\
\hline $\begin{array}{l}* \\
\cdots \\
\cdots\end{array}$ & 18 & 72 & 59 & 64 & & & & 94 & 74 & $83\}$ & & 01 & 53 \\
\hline $\begin{array}{l}1 \\
\text { H. }\end{array}$ & 80 & 71 & 62 & 65 & 95. & 86. & 91. & 95 & 74 & 84 & & & \\
\hline$*$ & 21 & 74 & 61 & 66 & & & & 93 & 72 & 80 & 76 & 76 & 76 \\
\hline$*$ & 22 & 78 & 57 & 67 & & & & 86 & 71 & 74 & & & 10 \\
\hline$"$ & 23 & 79 & 60 & 69 & 98. & 96. & 97. & 78 & 59 & $70^{\circ}$ & & & \\
\hline$" *$ & 24 & 80 & 67 & 73 & & & & 83 & 56 & 70 & 88 & 88 & 88 \\
\hline w & 85 & 72 & 54 & 63 & & & & 87 & 62 & 87 & & & \\
\hline$\ddot{*}$ & 26 & 69 & 53 & 62 & 97. & 100. & 98. & 80 & 63 & 71 & & & \\
\hline$\ddot{\theta}$ & 27 & 74 & 61 & 66 & & & & 70 & 62 & 67 & 95 & 92 & 94 \\
\hline $\begin{array}{l}14 \\
4 \quad 2\end{array}$ & 28 & 75 & 53 & 64 & & & & 75 & 53 & 64 & & & \\
\hline$\because 2$ & 29 & 71 & 59 & 64 & 100. & 100. & 100. & 77 & 50 & 63 & & & \\
\hline 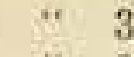 & 30 & 65 & 55 & 60 & & & & 80 & 53 & 65 & 100 & 99 & 99 \\
\hline * 3 & 31 & 70 & 46 & 56 & & & & 85 & 57 & 69 & & & \\
\hline Sept. & 1 & 74 & 49 & 61 & 100. & 100 & 100. & 76 & 63 & 69 & 100 & 100 & 100 \\
\hline ". & $\frac{2}{3}$ & $\begin{array}{l}82 \\
80\end{array}$ & 56 & $\begin{array}{l}68 \\
69\end{array}$ & & & & 82 & 65 & 71 & & & \\
\hline$\ddot{*}=$ & $\begin{array}{l}3 \\
4\end{array}$ & $\begin{array}{l}80 \\
81\end{array}$ & $\begin{array}{l}58 \\
62\end{array}$ & $\left.\begin{array}{l}69 \\
69\end{array}\right\}$ & 100. & 100. & 100. & $\begin{array}{l}82 \\
85\end{array}$ & 62 & 72 & & & \\
\hline 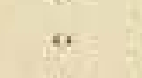 & 5 & 75 & $\begin{array}{l}62 \\
63\end{array}$ & 69 & & & & $\begin{array}{l}85 \\
89\end{array}$ & $\begin{array}{l}61 \\
71\end{array}$ & $\begin{array}{l}72 \\
79\end{array}$ & 100 & 100 & 100 \\
\hline 4 & 6 & 80 & 61 & 69 & 99. & 99. & & 90 & 68 & 74 & & & \\
\hline 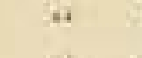 & 7 & 84 & 67 & 73 & . & & 99. & 87 & 72 & 78 & & & \\
\hline " & 8 & 87 & 68 & 77 & & & & 78 & 68 & 72 & 100 & 100 & 100 \\
\hline 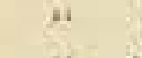 & 9 & 85 & 67 & 76 & & & & 78 & 56 & 68 & & & \\
\hline " 1 & 10 & 87 & 68 & 77 & 100. & 100. & 100. & 82 & 58 & $68^{\circ}$ & & & \\
\hline " 1 & 11 & 89 & 67 & 77 & & & & 85 & 65 & 73 & 100 & 100 & 100 \\
\hline $\begin{array}{ll}11 & 1\end{array}$ & 12 & 89 & 70 & 79 & & & & 82 & 68 & 75 & & & \\
\hline$"$ & 13 & 90 & 71 & 79 & 100. & 100. & 100. & 70 & 62 & 68 & & & \\
\hline "1. 1 & 14 & 90 & 75 & 81 & & & & 75 & 60 & 65 & 100 & 100 & 100 \\
\hline ". 1 & 15 & 89 & 74 & $79^{\circ}$ & & & & 63 & 47 & 58 & & & \\
\hline "1 1 & 16 & 89 & 72 & 76 & 100. & 100. & 100 . & 68 & 35 & 50 & & & \\
\hline+1 & 17 & 82 & 70 & 75 & & & & 76 & 47 & 59 & 100 & 100 & 100 \\
\hline " 1 & 18 & 85 & 69 & 74 & & & & 64 & 40 & 55 & & & \\
\hline .. 1 & 19 & 84 & 40 & 71 & 100. & 100. & 100. & 70 & 35 & $\begin{array}{l}51\} \\
56\}\end{array}$ & 100 & 100 & 100 \\
\hline ". & 20 & 70 & 41 & 67 & & & & 77 & 41 & 567 & & & \\
\hline
\end{tabular}


slight influence. Our plan, therefore, was to make a study of our data with reference to temperature alone, and find as nearly as possible the temperature constant for each of the stages; then to study the data with reference to humidity and evaporation and ascertain what effects different humidities or different evaporation rates had on the temperature constant. Several statements have already been made to the effect that the influence of humidity and evaporation is too slight to make it necessary to take them into account. It remains now only to present the data upon which this conclusion was based.

It is hardly to be expected that the degree of humidity of the atmosphere or the rate of evaporation could have any effect on the larva, surrounded as it is by the juicy substance of the apple; therefore the data bearing upon the relation of these factors to the rate of larval development have not been brought together; but the data bearing upon their relation to the rate of development of the egg and pupa are summarized in the following tables (47-50). The ranges of temperature are given at the top of the tables and the ranges of humidity or the rate of evaporation in the first column at the left, and then, in their proper places, are inserted the average effective day-degrees of all the individuals whose periods were observed at the respective temperatures and humidities or rates of evaporation.

In the right-hand column are given the average effective day-degrees for the different degrees of humidity or evaporation regardless of temperature; and at the bottom of the tables, the average effective day-degrees for the different temperatures, regardless of humidity or evaporation. The smaller figures, above the numbers indicating the average effective day-degrees, record the number of observations on which the averages are based.

Tables $47-50$ show that there is no consistent variation in the average total effective day-degrees corresponding to the different degrees of humidity or rates of evaporation. The variations are rather hit and miss, apparently depending more upon the number of individuals upon which the averages were based than upon any other factor. In all cases where the number of observations on which the averages were based is large, the average effective day-degrees vary only slightly from the total average. Possibly there is some slight indication that incubation proceeds at a greater rate at humidities between 70 and 74 than at higher or lower humidities and at rates of evaporation between 20 and 24 than at lower rates; but we are hardly warranted in basing any definite conclusion on this indication, and if we were, the variations are so slight that for practical purposes they could be disregarded, since the extreme variations where the number of observations from the average are 500 or more would amount to a variation of less than one eighth of a day in the period. 
TABLE 47-Relation of Humidity to Rate of Development of Ege AS SHOWN BY EFFECTIVE DAY-DEGREES

\begin{tabular}{|c|c|c|c|c|c|c|}
\hline $\begin{array}{c}\text { Temp. } \\
\text { Humid. } \\
\text { 举 }\end{array}$ & $60^{\circ}-64^{\circ}$ & $65^{\circ}-69^{\circ}$ & $70^{\circ}-74^{\circ}$ & $75^{\circ}-79^{\circ}$ & $80^{\circ}-84^{\circ}$ & Average \\
\hline $\begin{array}{c}65-69 \\
70-74 \\
75-79 \\
80-84 \\
85-89\end{array}$ & $\begin{array}{r}138 \\
171.2 \\
53 \\
153.0 \\
169 \\
156.3 \\
111 \\
172.0 \\
132 \\
171.3\end{array}$ & $\begin{array}{l}81 \\
164.3 \\
847 \\
164.5 \\
237 \\
159.6 \\
38 \\
174.4\end{array}$ & $\begin{array}{c}251 \\
161.4 \\
703 \\
162.9 \\
282 \\
160.8 \\
70 \quad 172.0\end{array}$ & $\begin{array}{l}114 \\
161.0 \\
275 \\
160.3 \\
159 \\
162.6 \\
52 \\
163.0\end{array}$ & $\begin{array}{c}256 \\
163.4 \\
206 \\
162.7 \\
13 \\
175.0\end{array}$ & $\begin{array}{c}138 \\
171.2 \\
755 \\
161.7 \\
2200 \\
162.6 \\
802 \\
162.6 \\
292 \\
170.4\end{array}$ \\
\hline Average & ${ }^{593} 165.6$ & $\begin{array}{l}1203 \\
165.1\end{array}$ & $\begin{array}{l}1306 \\
162.6\end{array}$ & $\begin{array}{l}600 \\
161.3\end{array}$ & $\begin{array}{l}475 \\
\quad 163.4\end{array}$ & $\begin{array}{l}4187 \\
163.3\end{array}$ \\
\hline
\end{tabular}

TABLE 48-Relation of Evaporation to Rate of Development of Egg AS SHOWN BY EFFECTIVE DAY-DEgREES

\begin{tabular}{|c|c|c|c|c|c|c|}
\hline $\begin{array}{c}\text { Temp. } \\
\text { Evap. } \\
\text { 糞 }\end{array}$ & $60^{\circ}-64^{\circ}$ & $65^{\circ}-69^{\circ}$ & $70^{\circ}-74^{\circ}$ & $75^{\circ}-79^{\circ}$ & $80^{\circ}-84^{\circ}$ & Average \\
\hline $\begin{array}{c}5-9 \\
10-14 \\
15-19 \\
20-24 \\
25-29\end{array}$ & $\begin{array}{r}32 \\
150.0 \\
398 \\
165.8 \\
71 \\
159.3 \\
102 \\
172.9\end{array}$ & $\begin{array}{l}85 \\
160.9 \\
807 \\
164.4 \\
311 \\
163.1\end{array}$ & $\begin{array}{r}217 \\
160.4 \\
431 \\
165.8 \\
409 \\
160.8 \\
249 \\
161.3\end{array}$ & $\begin{array}{c}353 \\
161.4 \\
47 \\
166.5 \\
177 \\
157.6 \\
23 \\
176.2\end{array}$ & $\begin{array}{c}181 \\
166.2 \\
112 \\
156.0 \\
182 \\
165.4\end{array}$ & $\begin{array}{c}834 \\
159.9 \\
1989 \\
164.5 \\
1019 \\
162.6 \\
640 \\
161.2 \\
205 \\
166.6\end{array}$ \\
\hline Average & ${ }^{603} 165.6$ & $\begin{array}{c}1203 \\
163.9\end{array}$ & $\begin{array}{l}1308 \\
162.5\end{array}$ & $\begin{array}{l}600 \\
161.2\end{array}$ & ${ }^{475} 163.5$ & $\begin{array}{l}4187 \\
163.3\end{array}$ \\
\hline
\end{tabular}


TABLE 49-Relation of Humidity to Rate of Development of Pupa AS SHOWN BY EFFECTIVE DAY-DEGREES

\begin{tabular}{|c|c|c|c|c|c|c|c|c|}
\hline $\begin{array}{l}\text { Temp.s. } \\
\text { Humid. }\end{array}$ & $50^{\circ}-54^{\circ}$ & $55^{\circ}-59^{\circ}$ & $60^{\circ}-64^{\circ}$ & $65^{\circ}-69^{\circ}$ & $70^{\circ}-74^{\circ}$ & $75^{\circ}-79^{\circ}$ & $80^{\circ}-84^{\circ}$ & Average \\
\hline $\begin{array}{c}60-64 \\
65-69 \\
70-74 \\
75-79 \\
80-84\end{array}$ & $\stackrel{42}{236.2}$ & $\begin{array}{l}508 \\
238.7 \\
955 \\
240.9\end{array}$ & & $\begin{array}{l}31 \\
236.0 \\
187 \\
234.7\end{array}$ & $\begin{array}{l}55 \\
237.0 \\
34 \\
247.2 \\
125 \\
248.3 \\
339 \\
235.0\end{array}$ & $\begin{array}{l}163 \\
238.3 \\
588 \\
242.7 \\
357 \\
237.3\end{array}$ & $\begin{array}{l}2 \\
254.0 \\
369 \\
244.4 \\
18 \\
239.9\end{array}$ & $\begin{array}{l}565 \\
238.6 \\
1563 \\
241.5 \\
760 \\
243.3 \\
863 \\
235.9\end{array}$ \\
\hline Average & $\begin{array}{l}42 \\
236.2\end{array}$ & $\begin{array}{l}1463 \\
240.1\end{array}$ & & $\begin{array}{l}198 \\
234.4\end{array}$ & $\begin{array}{l}553 \\
239.0\end{array}$ & $\begin{array}{l}1106 \\
240.6\end{array}$ & $\begin{array}{l}389 \\
244.2\end{array}$ & $\begin{array}{l}3751 \\
240.1\end{array}$ \\
\hline
\end{tabular}

TABle 50-Relation of Evaporation to Rate of Development of Pupa AS SHOWN BY EFFECTIVE DAY-DEGREES

\begin{tabular}{|c|c|c|c|c|c|c|c|}
\hline $\begin{array}{c}\text { Temp. } \\
\text { Evap. } \\
\text { y }\end{array}$ & $50^{\circ}-54^{\circ}$ & $55^{\circ}-59^{\circ}$ & $65^{\circ}-69^{\circ}$ & $70^{\circ}-74^{\circ}$ & $75^{\circ}-79^{\circ}$ & $80^{\circ}-84^{\circ}$ & Average \\
\hline $\begin{array}{c}5-9 \\
10-14 \\
15-19 \\
20-24 \\
25-29\end{array}$ & $\stackrel{42}{236.0}$ & $\begin{array}{c}1077 \\
240.2 \\
174 \\
243.7 \\
\\
212 \\
237.1\end{array}$ & $\begin{array}{l}52 \\
237.9 \\
146 \\
233.3\end{array}$ & $\begin{array}{c}{ }^{6} 224.1 \\
228 \\
395 \\
236.4 \\
70 \\
251.4 \\
34 \\
241.4 \\
48 \\
238.7\end{array}$ & $\begin{array}{l}380 \\
238.3 \\
480 \\
242.6 \\
199 \\
239.1 \\
47 \\
235.0\end{array}$ & $\begin{array}{c}16 \\
239.0 \\
363 \\
244.5\end{array}$ & $\begin{array}{c}1177 \\
239.8 \\
1095 \\
237.8 \\
550 \\
244.5 \\
481 \\
238.3 \\
458 \\
242.9\end{array}$ \\
\hline Average & $\stackrel{42}{236.0}$ & $\begin{array}{c}1463 \\
240.1\end{array}$ & $\begin{array}{l}198 \\
234.4\end{array}$ & $\begin{array}{l}\mathrm{s} 53 \\
239.0\end{array}$ & $\begin{array}{l}1106 \\
240.6\end{array}$ & $\begin{array}{l}379 \\
244.2\end{array}$ & $\begin{array}{c}3741 \\
240.1\end{array}$ \\
\hline
\end{tabular}




\section{Spray Program for the Codling-moth}

Spray programs heretofore outlined have been based on fixed dates or on time of the fall of the petals. It is better to follow programs of these kinds than to spray hit and miss, but these programs do not take into consideration seasonal variations and variations due to local peculiarities, to altitude, to latitude, or to other circumstances which might have an effect upon temperature.

The following program is based upon the relation of accumulated effective day-degrees to the time of the appearance of the different broods of larvæ. It provides for seven sprays, three for the first brood, two for the second, and two for the third. The table shows the accumulation of effective day-degrees at which the fruit-grower should begin to spray in order that he may complete the work in from one to ten days. This spray program (Table 51), like all others which have been proposed, must be tested thoroughly and proved reliable before it can be accepted as satisfactory.

Too much emphasis can not be placed upon the necessity of applying two sprays for the second brood of larvæ, and of making the first spray for the second brood very thorough. The second brood is much more numerous than the first and comes at a time when the fruit is increasing in size very rapidly; hence, one spray applied at the time when the first larvæ of the second brood enter the fruit will not suffice to check those that enter three or four weeks later. The second and third broods of larvæ overlap, consequently after the second brood begins to enter the fruit larvæ are entering it continuously in large numbers till all the thirdbrood larvæ have entered, hence the fruit should be well protected during this period.

The first larvæ of the second generation which enter the fruit during July transform and produce the third generation; hence the importance of making the first spray for the second brood (the fourth spray on the program) very thorough so as to destroy as many of the first larva of this brood as possible. To reduce the third brood still further the spraying should be supplemented by the destruction of larvæ under bands up to the first of September. Larvæ that leave the fruit after that date will not transform until the following spring, and may be destroyed any time after the season closes.

The seventh spray, or the second spray for the third brood, will not be necessary in case the sixth spray comes late in August. If it should come prior to the 15th of August the seventh spray will be advisable unless the latter part of August should be much cooler than normal. 
TABLE 51-Spray Program

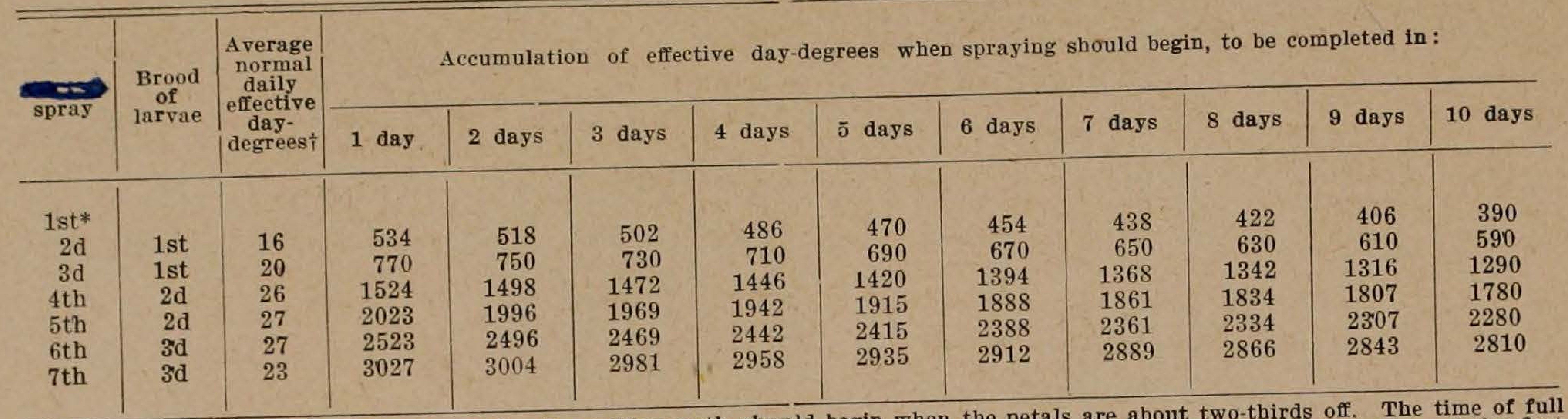

* The application of the first spray for the codling-moth should begin when the petals are about two-thirds off. The time of full bloom is not dependent upon effective day-degrees as deffective day-degrees.

spray can not be determined by the accumulation of t The figures in this colum 